\title{
ADVANCEMENTS IN MULTI-OBJECTIVE ECO-ROUTING SOLUTIONS USING CONNECTIVITY
}

\section{By}

\section{Lama Alfaseeh}

Master in Engineering, Damascus University, February 2011

Bachelor in Civil Engineering, Damascus University, September 2006

\author{
A dissertation \\ presented to Ryerson University \\ in partial fulfillment of the \\ requirements for the degree of \\ Doctor of Philosophy \\ in the program of \\ Civil Engineering
}

Toronto, Ontario, Canada, 2020

(C) Copyright Lama Alfaseeh, 2020 
(c) Copyright by LAMA ALFASEEH, 2020

All Rights Reserved 


\section{ACKNOWLEDGMENT}

\section{"For anyone who does not taste the bitterness of acquiring knowledge, he/she will taste the humiliation of ignorance all his/her life" Shafi'i}

First and foremost, I would like to thank God Almighty for giving me the strength, determination, ability, and patience to undertake this research. Without his blessings, this achievement would not have been possible.

I am deeply indebted to my parents for raising me to value learning and academic achievements. I was nurtured not to waste time and to take advantage of opportunities. My mom, Prof. Khetam, and dad, Dr. Ayman, are absolutely my role models.

I would also like to extend my deepest gratitude to Dr. Bilal Farooq, my supervisor, for his continuous support during my $\mathrm{PhD}$ journey. I remember when I first met him, he told me that he only researches cool stuff. Now, I know exactly what he meant! I was extremely fortunate to work under his supervision. He is a very ambitious, knowledgeable, respectful, supportive, and understanding supervisor. Dr. Bilal is what every PhD student would ask for.

I would like to express my deepest appreciation to my committee members: Dr. Hanna Maoh, Dr. Marianne Hatzopoulou, Dr. Tor Oiamo, Dr. Medhat Shehata, and Dr. Sabbir Saiyed. They have generously dedicated their time to be part of my committee and provide constructive feedback.

The completion of my dissertation would not have been possible without the support and love of my husband, friend, and partner, Houssam Loutfi. He celebrated my success and encouraged me when I needed a push. He believed in me and was sure that I would make it.

Being a mom and a PhD student was tough, but the inspiration I got from my kids, 
Hamzah (my heart) and Sima (my soul), made it much easier and made me more resilient to the challenges I faced.

I would like to thank my sister, Dima, and brother, Ghaleb, for their encouragement and support.

I would also like to extend my deepest gratitude to Dr. A for his special type of support that made this work happen.

I would like to thank Prof. El-Rabbany for his support during my PhD journey from day one until the end. His door was always open for me, regardless of the issue. His advice definitely contributed positively to my professional and academic achievements. Prof. ElRabanny is absolutely an asset to Ryerson University. My sincere thanks are also to Prof. Miljana Horvat for her support.

I am extremely grateful to Prof. Omar Hefni who shared priceless advice, followed up on my progress, and supported me in several ways throughout this journey.

I would like to thank my research mates at the Laboratory of the Innovations in Transportation (LiTrans). The friendly environment, Dr. Bilal encouraged, helped reducing the stress level during my $\mathrm{PhD}$ journey. All of the research team members were ready to assist when they were asked. My sincere thanks is for Dr. Shadi, who worked closely with me and absolutely contributed to this research work. Thanks to Dr. Melvin, Arash, Rafael, Veronica, and Mehdi for being supportive colleagues. Outside of LiTrans, I would like to thank Yasmine and Markus Jesswein for being amazing colleagues.

Last but certainly not least, many thanks to my friends, who believed in me, especially Abeer Dandal who was always there for me even when I did not ask for help. 


\title{
ADVANCEMENTS IN MULTI-OBJECTIVE ECO-ROUTING SOLUTIONS
}

\section{USING CONNECTIVITY}

\author{
Abstract \\ by Lama Alfaseeh \\ Doctor of Philosophy, 2020 \\ Civil Engineering \\ Ryerson University
}

Due to the significant adverse impact of transportation systems on the environment, topics related to alleviating greenhouse gas $(\mathrm{GHG})$ emissions are gaining more attention. As potential solutions to mitigate GHG emissions, several approaches have been proposed to better control traffic and manage transportation systems. The employment of Intelligent Transportation System (ITS), which adopts the advancements in Information and Communication Technology (ICT), has been proposed as the most favourable approach to alleviate the undesirable impact of transportation systems on the environment. ITS can control several aspects of a network, such as speed, traffic signals, and route guidance. For the purpose of routing, this research aims to exploit the advancements in ICT by including connected and automated vehicles (CAVs) and sensing technology in an urban congested network.

Anticipatory multi-objective eco-routing in a distributed routing framework was proposed and compared to myopic routing with a large case study on a congested network. The End-to-End Connected Autonomous Vehicles (E2ECAV) dynamic distributed routing framework was examined, and encouraging results were found based on the traffic and environmental perspectives. The impact of different market penetration rates (MPRs) of CAVs was examined for various traffic conditions. E2ECAV was adopted for both the myopic and 
anticipatory routing strategies in this dissertation. The best GHG costing approach was defined and was among the elements tackled in this research. For a robust anticipatory routing application, predictive models were developed based on Long-Short Term Memory (LSTM), a deep learning approach, while considering a high level of spatial (link level) and temporal (one minute) resolution. With regards to the LSTM predictive models, the impact was illustrated of using a deeper LSTM network and systematically tuning its hyper-parameters. The anticipatory routing strategy significantly outperformed the myopic routing strategy based on the the traffic and environmental perspectives. This research shows that ITS can help significantly reduce GHG emissions produced by transportation systems. The developed predictive models can be used while real-time data are collected from sensors within an urban network. Furthermore, the proposed anticipatory routing framework can be applied in a real-time situation. 


\section{TABLE OF CONTENTS}

Page

ACKNOWLEDGMENT ...................... ii

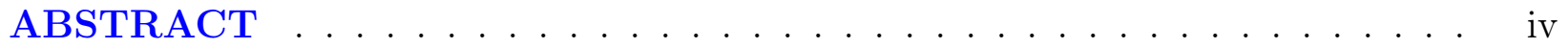

LIST OF TABLES ....................... . ix

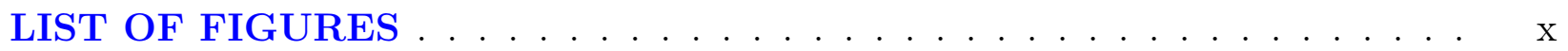

CHAPTER

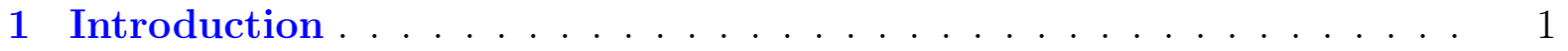

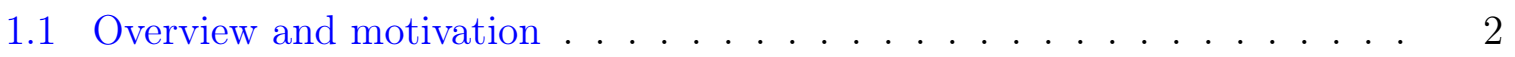

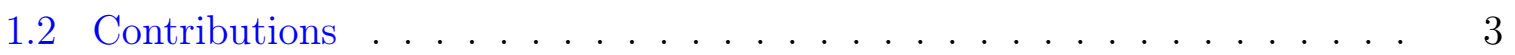

1.3 Research Significance . . . . . . . . . . . . . . . . . . . . 4

1.4 Dissertation Outline . . . . . . . . . . . . . . . . . 5

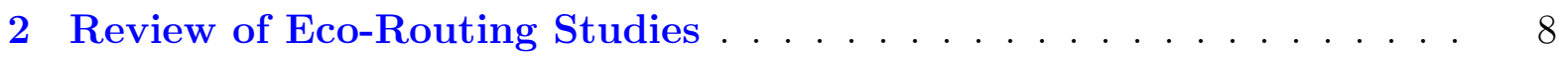

2.1 Introduction . . . . . . . . . . . . . . . . . . . . . . . . . 9

2.2 Vehicle Routing, Eco-Routing, and Traffic Assignment . . . . . . . . . 10

2.3 Vehicle Emission Modelling . . . . . . . . . . . . . . . . . . . . 17

2.3.1 Static Emission Models . . . . . . . . . . . . . . . . . . 18

2.3.2 Dynamic Emission Models . . . . . . . . . . . . . . . . . . . 19

2.4 Taxonomy of the Eco-Routing Studies . . . . . . . . . . . . . 20

2.5 Classification of Existing Literature . . . . . . . . . . . . . . 22

2.5 .1 Microscopic (I) Models . . . . . . . . . . . . . . . 22

2.5 .2 Mesoscopic (E) Models . . . . . . . . . . . . . 26

2.5 .3 Macroscopic (A) Models . . . . . . . . . . . . 26

2.5.4 Mixed Aggregation (M) Models . . . . . . . . . . . . 31

2.6 Concluding Remarks . . . . . . . . . . . . . . . . . . . . . . . . 34 
3 Background Components . . . . . . . . . . . . . . . . . 36

3.1 Introduction . . . . . . . . . . . . . . . . . . . 37

3.2 Vehicle Routing Framework _ . . . . . . . . . . . . . . 37

3.3 Traffic Simulator . . . . . . . . . . . . . . . . . . . 40

3.4 Emission Simulator . . . . . . . . . . . . . . . . . . . 43

3.5 Case Study . . . . . . . . . . . . . . . . . . . . . . . . . . 45

4 Analysis of End-to-End Connected Automated Vehicles (E2ECAV) . 51

4.1 Introduction . . . . . . . . . . . . . . . . . . . . . . 52

4.2 Related Literature . . . . . . . . . . . . . . . . . . . 56

4.3 Traffic and Emission Models . . . . . . . . . . . . . . . . . 62

4.4 Case Study . . . . . . . . . . . . . . . . . . . . . . . . 63

4.5 Description of E2ECAV . . . . . . . . . . . . . . . . . 63

4.6 E2ECAV Parameters Analysis . . . . . . . . . . . . . . . . . 64

4.6 .1 Updating Interval . . . . . . . . . . . . . . . . . 64

4.6 .2 IDM Parameters . . . . . . . . . . . . . . . . . . . 66

4.6.3 Shortest Paths (K-Paths) . . . . . . . . . . . . . . . 68

4.7 Results and Discussion Using Optimal Parameters . . . . . . . . . . . 69

4.7.1 E2ECAV Traffic Analysis . . . . . . . . . . . . . . . . . . . 69

4.7.2 E2ECAV Environmental Analysis . . . . . . . . . . . . 83

4.8 Concluding Remarks . . . . . . . . . . . . . . . . . . . . . . 85

5 Myopic Eco-Routing _ . . . . . . . . . . . . . . . . . . . . . 87

5.1 Introduction $\ldots \ldots \ldots \ldots \ldots \ldots \ldots \ldots \ldots \ldots \ldots \ldots \ldots \ldots$

5.2 Literature . . . . . . . . . . . . . . . . . . . . . . . . . 89

5.3 Traffic and Emission Models . . . . . . . . . . . . . . . . . . 92

5.4 Case Study . . . . . . . . . . . . . . . . . . . . . . . . . . . . . . . . 93

5.5 GHG Costing Approaches _. . . . . . . . . . . . . . . . . . . 93

5.6 Routing Strategies . . . . . . . . . . . . . . . . . . . . . . . . . 95

5.7 Results and Analysis . . . . . . . . . . . . . . . . . . 97

5.7.1 GHG Costing Approach Analysis . . . . . . . . . . . . . . 97

5.7.2 Average TT, Average VKT, Total GHG, and Total NOx Analysis of the Different Myopic Routing Strategies _. . . . . . . . . 100

5.7.3 Path Analysis of the Different Myopic Routing Strategies . . . . 102

5.7.4 Network Level Analysis of the Different Myopic Routing Strategies 103

5.8 Concluding Remarks . . . . . . . . . . . . . . . . . . . . . 106 
6 Prediction Models . . . . . . . . . . . . . . . . . . . . . 108

6.1 Introduction . . . . . . . . . . . . . . . . . . . . . . . . 109

6.2 Literature . . . . . . . . . . . . . . . . . . . . . 110

6.3 ARIMA with Exogenous Variables . . . . . . . . . . . . . . . . . 114

6.4 Clustering . . . . . . . . . . . . . . . . . . . . . . 115

6.5 LSTM with Exogenous Variables . . . . . . . . . . . . . . . . 117

6.6 Data Collection . . . . . . . . . . . . . . . . . . . . . . . . . 118

6.7 Results and Discussion . . . . . . . . . . . . . . . . . . 121

6.7 .1 Correlation Analysis . . . . . . . . . . . . . . . . . . . . 121

6.7 .2 GHG ER Models . . . . . . . . . . . . . . . . . . 124

6.7 .3 Speed Model . . . . . . . . . . . . . . . . . . . . . 133

6.8 Concluding Remarks . . . . . . . . . . . . . . . . . . . . . . . . . 134

7 Anticipatory Eco-Routing . . . . . . . . . . . . . . . 137

7.1 Introduction . . . . . . . . . . . . . . . . . . . . . . 138

7.2 Literature . . . . . . . . . . . . . . . . . . . . . . 140

7.3 Traffic and Emission Models . . . . . . . . . . . . . . . . . 145

7.4 Case Study . . . . . . . . . . . . . . . . . . . . . . . . . . . . . . 145

7.5 Predictive Models . . . . . . . . . . . . . . . . . . . . . 146

7.6 Discussion . . . . . . . . . . . . . . . . . . . . . . 146

7.6.1 Average TT, Average VKT, Total GHG, and Total NOx by the Different Routing Strategies . . . . . . . . . . . . . . 148

7.6.2 Path Analysis of the Different Myopic and Anticipatory Routing Strategies . . . . . . . . . . . . . . . . . . . 151

7.6.3 Network Level Analysis of Average Speed, GHG, and NOx Over Time from the Routing Strategies . . . . . . . . . . . . . . 153

7.7 Concluding Remarks . . . . . . . . . . . . . . . . . . 157

8 Summary, Concluding Remarks, and Future Outlook . . . . . . . . 159

8.1 Summary . . . . . . . . . . . . . . . . . . . . 160

8.2 Concluding Remarks . . . . . . . . . . . . . . . . . . . . . 165

8.3 Future Outlook . . . . . . . . . . . . . . . . . . . . . . 171

REFERENCES . . . . . . . . . . . . . . . . . . . . . . . . . 187 


\section{LIST OF TABLES}

2.1 Acronyms and Abbreviations Used in Chapter Two . . . . . . . . . . . 11

2.2 Eco-Routing Models and Their Specifications Related to the Proposed ThreeFactor Taxonomy . . . . . . . . . . . . . . . . . . . . 28

3.1 Operating Modes Based on VSP Bins . . . . . . . . . . . . . . 46

3.2 Characteristics of the Links in the Case Study . . . . . . . . . . . 50

4.1 The Investigated Input Parameters _ . . . . . . . . . . . . . 65

5.1 The Investigated GHG Costing Strategies _ . . . . . . . . . . . . . 93

5.2 The Considered Routing Strategies _ . . . . . . . . . . . . . . . . 97

6.1 The Considered Scenarios for Data Generation . . . . . . . . . . . . . 120

6.2 Trained LSTM Network and their specifications . . . . . . . . . . . 128

6.3 Performance Indicators of the Trained LSTM Networks . . . . . . . . . . . 131

7.1 The Investigated Routing Strategies . . . . . . . . . . . . . . . . 148 


\section{LIST OF FIGURES}

1.1 Dissertation Framework $\ldots \ldots \ldots \ldots$

2.1 Traffic Assignment Classification, Components, and Traffic Assignment Relationship with Routing (Alfaseeh and Farooq, 2020b) . . . . . . . . . . 12

2.2 Eco-Routing Framework (Alfaseeh and Farooq, 2020b) . . . . . . . 16

2.3 Classification of Vehicle Emission Models (Alfaseeh and Farooq, 2020b) . . 17

2.4 Abbreviations of the Proposed Three Factor Taxonomy (Alfaseeh and Fa-

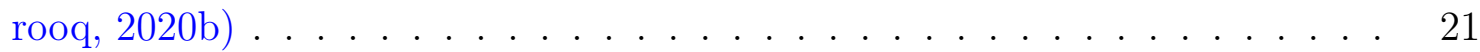

3.1 Diagram of the Traffic and Emission Models and Their Interactions with the Vehicle Routing Framework in This Study . . . . . . . . . . . . . . . 39

3.2 Emission Rates Versus Different OpModes for Two Different Vehicle Type . . 45

3.3 Operation of the Emission Simulator and its Relationship with the Traffic Simulator . . . . . . . . . . . . . . . . . . . . . . 47

3.4 Case Study of Downtown Toronto, Ontario, Canada . . . . . . . . . . . . 49

4.1 Centralized Versus Distributed Routing System _ . . . . . . . . . . . 54

4.2 Chapter Four Framework . . . . . . . . . . . . . . . . . . 56

4.3 Automation Levels of Vehicles (Society of Automotive Engineers, 2018) . . 58 
4.4 The Impact of Different Updating Intervals on the a) Mean TT, Mean VKT and b) Throughput . . . . . . . . . . . . . . . . . 66

4.5 The Impact of Different IDM Sets on the a) Mean TT, Mean VKT, and b)

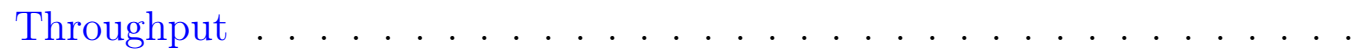

4.6 The Impact of the Number of Optimal K-Paths on the a) Mean TT, Mean VKT, and b) Throughput . . . . . . . . . . . . . . . 68

4.7 Mean TT (bars) and Mean VKT (lines) for Different MPRs of CAVs and Traffic Conditions . . . . . . . . . . . . . . . . . . .

4.8 The Throughput for the a) Highly Congested, b) Congested, c) Uncongested

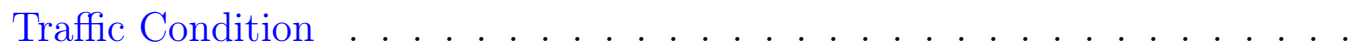

4.9 Fundamental Diagrams of a) Speed (km/h) vs. Density (veh/km/ln), b) Flow (veh/h) vs. Speed $(\mathrm{km} / \mathrm{h})$, and c) Flow (veh/h) vs. Density (veh/km/ln) for the Most Congested Link under the Highly Congested Traffic Condition and Different MPRs of CAVs . . . . . . . . . . . . . . . . 76

4.10 Fundamental Diagrams of a) Speed (km/h) vs. Density (veh/km/ln), b) Flow (veh/h) vs. Speed $(\mathrm{km} / \mathrm{h})$, and c) Flow (veh/h) vs. Density $(\mathrm{veh} / \mathrm{km} / \mathrm{ln})$ for the Most Congested Link under the Congested Traffic Condition and Different

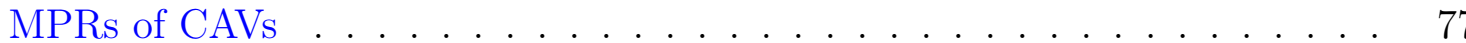

4.11 Fundamental Diagrams of a) Speed $(\mathrm{km} / \mathrm{h})$ vs. Density (veh/km/ln), b) Flow (veh/h) vs. Speed $(\mathrm{km} / \mathrm{h})$, and c) Flow $(\mathrm{veh} / \mathrm{h})$ vs. Density $(\mathrm{veh} / \mathrm{km} / \mathrm{ln})$ for the Most Congested Link under the Uncongested Traffic Condition and Different MPRs of CAVs .................... 78

4.12 The Most Congested Link in the Network under the Highly Congested Traffic Condition and its a) Speed, b) Density, c) Flow . . . . . . . . . . . . . . 80 
4.13 The Most Congested Link in the Network under the Congested Traffic Condition and its a) Speed, b) Density, c) Flow . . . . . . . . . . . . . . 81

4.14 The Most Congested Link in the Network under the Uncongested Traffic Condition and its a) Speed, b) Density, c) Flow . . . . . . . . . . . . . 82

4.15 The Impact of Different MPRs of CAVs and Traffic Conditions on the a) Total GHG and b) Total NOx ...................... . . . 84

5.1 Chapter Five Framework . . . . . . . . . . . . . . . . . . . . . . . . . . . 90

5.2 The Impact of Different GHG Costing Approaches on the a) Mean TT and VKT and b) Total GHG and NOx . . . . . . . . . . . . . . 98

5.3 The Impact of the Different Myopic Routing Strategies on the a) Mean TT and VKT and b) Total GHG and NOx . . . . . . . . . . . . . . 101

5.4 Myopic Routes of a Random Vehicle of the Different Routing Strategies, a) TT, b) GHG, and c) TT\&GHG . . . . . . . . . . . . . . . 104

5.5 The Network under the Different Myopic Routing Strategies and its a) Speed, b) GHG, and c) NOx over time . . . . . . . . . . . . . . 105

6.1 Chapter Six Framework . . . . . . . . . . . . . . . . . . . 111

6.2 Methodology for a) ARIMA with Exogenous Variables, b) Clustering, and c) LSTM with Exogenous Variables . . . . . . . . . . . . . . . 116

6.3 Histogram of a) Speed, b) Density per Lane, c) Flow, and d) GHG ERs (in $\left.\mathrm{CO}_{2 \mathrm{eq}} \mathrm{g} / \mathrm{sec}\right) \ldots \ldots \ldots \ldots \ldots \ldots$

6.4 Correlation between the Proposed Variables at Every Minute from 1 to 5 (Top to Bottom) with a) GHG ER and b) Speed at the 6th Minute . . . . . . . 122

6.5 Sample Representation (4 Sampled Links) of Network Level Prediction Using ARIMA with Exogenous Variables 
6.6 Sum of Squared Error (Elbow Method) of 15 Clusters . . . . . . . . . . . 126

6.7 True vs. Predicted GHG ERs (in $\mathrm{CO}_{2 \mathrm{eq}} \mathrm{g} / \mathrm{sec}$ ) of Clustering Where: a) is for 5 Clusters, b) is for 10 Clusters, and c) is for 15 Clusters . . . . . . . . . 127

6.8 True vs. Predicted GHG ERs (in $\mathrm{CO}_{2 \text { eq }} \mathrm{g} / \mathrm{sec}$ ) of a) $\mathrm{LSTM}_{m}$ and b) $\mathrm{LSTM}_{b} 130$

6.9 True vs. Predicted GHG ERs (in $\mathrm{CO}_{2 \text { eq }} \mathrm{g} / \mathrm{sec}$ ) of a) LSTM2 $2_{m}$ and b) LSTM2 $2_{b} 131$

6.10 True vs. Predicted GHG ERs (in $\mathrm{CO}_{2 \text { eq }} \mathrm{g} / \mathrm{sec}$ ) of a) LSTM3 ${ }_{m}$ and b) LSTM3 ${ }_{b} 132$

6.11 True vs. Predicted Speed $(\mathrm{km} / \mathrm{h})$ of a) $\mathrm{LSTM}_{m}$ and b) $\mathrm{LSTM}_{b} \ldots \ldots$

7.1 Chapter Seven Framework . . . . . . . . . . . . . . . . . 139

7.2 The Impact of Myopic and Anticipatory Routing Strategies on the a) Mean TT and VKT and b) Total GHG and NOx . . . . . . . . . . . 150

7.3 Myopic Routes of a Random Vehicle Subjected to Different Routing Strategies: a) TT, b) GHG, and c) TT\&GHG . . . . . . . . . . . . . . . 152

7.4 Anticipatory Routes of a Random Vehicle Subjected to Different Routing Strategies: a) TT, b) GHG, and c) TT\&GHG . . . . . . . . . . . . . 153

7.5 The Network Under the Different Myopic Routing Strategies and its a) Speed, b) GHG, and c) NOx Over Time . . . . . . . . . . . . . . . 154

7.6 The Network Under the Different Anticipatory Routing Strategies and its a) Speed, b) GHG, and c) NOx Over Time . . . . . . . . . . . . . 155

8.1 Summary of the Stages Followed in this Dissertation From the Current Routing State to the Routing State in the Near Future . . . . . . . . . . . . . . . 161 


\section{Dedication}

This dissertation is dedicated to my parents, husband, and kids who supported me in different ways to accomplish this work. 
Chapter One

\section{Introduction}




\subsection{Overview and motivation}

Transportation systems have a dramatic undesirable impact on the environment. According to Environment and Climate Change Canada (2020), transportation system, which was the second largest source of greenhouse gas (GHG) emissions, contributed to $25 \%$ of the produced GHG emission in Canada in 2018. In 2017, it was found that $81 \%$ of the GHG was $\mathrm{CO}_{2}$, which is a major contributor to climate change and global warming (Z. Liu et al., 2020).

Several approaches have been proposed to alleviate the adverse impact of transportation systems on the environment. Substituting fossil fuel with other energy sources is one tool, but this is unlikely to be adopted completely in the near future. Improving vehicles' technology is another approach. The employment of intelligent transportation system (ITS), which utilizes the enhancements in Information and Communication Technology (ICT), has been suggested to mitigate GHG emissions. The use of ITS is potentially the most promising approach as it allows several measures to be controlled, such as speed, traffic signals, and route guidance (Zegeye et al., 2009). It has been shown that there is a proportional relationship between routing efficiency and the performance of a transportation system from the traffic and environmental perspectives (Djavadian, Tu, et al., 2020; Tu, Alfaseeh, et al., 2019; Alfaseeh, Djavadian, and Farooq, 2018; Yang and Recker, 2006). While routing vehicles to their destinations, ITS collects, processes, and shares data with users for a better performing transportation system (Council of Ministers Transportation and Highway Safety, 2012).

The employment of intelligent vehicles has contributed to favourable results from the traffic and environmental points of view as found in Djavadian, Tu, et al. (2020); Alfaseeh, Djavadian, Tu, et al. (2019); Tu, Alfaseeh, et al. (2019); Djavadian and Farooq (2018) and Alfaseeh, Djavadian, and Farooq (2018). With regards to sensing technologies as another form of ITS, distributed routing framework depends on different types of communication and has been proposed to overcome the limitations of the existing centralized routing framework. These limitations are related to the large investment required, high sensitivity to system failure, and 
high complexity in the case of system upgrades (Yang and Recker, 2006). For instance, the End-to-End Connected Automated Vehicles (E2ECAV) dynamic distributed routing framework (Farooq and Djavadian, 2019) has proven its ability to significantly outperform the centralized routing system, especially with high market penetration rates (MPRs) of CAVs (Djavadian, Tu, et al., 2020; Alfaseeh, Djavadian, Tu, et al., 2019; Tu, Alfaseeh, et al., 2019; Djavadian and Farooq, 2018; Alfaseeh, Djavadian, and Farooq, 2018). The existing routing systems, which are predominantly myopic, aim at minimizing travel time solely and are mostly applied in a centralized routing system (Alfaseeh and Farooq, 2020b). To incorporate the environmental dimension, eco-routing takes environmental variables into consideration while routing vehicles to their destinations and has replaced the original routing concept.

The motivation of this research is stemmed from the aforementioned facts related to the adverse effect that transportation systems imposed on the environment. In addition, the availability of supporting technologies for a better performing transport system represents another part of this dissertation's motivation. In this dissertation, the advancement in ICT, sensing and intelligent vehicles, were exploited to mitigate the undesirable impact of transportation systems on the environment. Novel anticipatory multi-objective eco-routing strategies were developed and applied in a distributed routing framework. Unlike myopic routing, which deals with the current traffic and environmental state, anticipatory routing depends on the predicted state and requires predictive models of the optimized variables.

\subsection{Contributions}

This dissertation aims to take advantage of the advancements in ICT to minimize the produced GHG emission while dynamically routing vehicles from their origins to their destinations. Routing was analyzed in different settings to minimize not only travel time but also greenhouse gas (GHG) emissions. To fulfill this objective, following steps were taken: 
- A distributed dynamic routing framework, End-to-End Connected Automated Vehicles (E2ECAV), was evaluated based on the traffic and environmental characteristics and its suitability in the context of eco-routing;

- Myopic single and multi-objective eco-routing strategies were developed as a base case;

- Predictive mechanisms based on Long Short-Term Memory (LSTM) model were developed;

- Anticipatory single and multi-objective eco-routing strategies were developed and compared with the base case, myopic routing strategies.

\subsection{Research Significance}

Anticipatory eco-routing strategies offer potential enhancements compared to myopic ecorouting strategies from the traffic and environmental perspectives. To exploit the advancement in ICT, the E2ECAV dynamic distributed routing system, which uses CAVs and two types of communication, was examined in detail and employed in this dissertation. Demonstrating the efficiency of the E2ECAV assures that connectivity contributes to significant enhancements from the traffic and environmental perspectives. This will encourage future research work to adopt distributed routing systems. To apply the anticipatory routing realistically, sophisticated predictive models were developed in this dissertation for the variables in concern. A high level of temporal (1 minute) and spatial (link level) resolution was adopted, despite the high level of complexity associated. The developed predictive models can be used while real-time data are provided from any urban network. In other words, if the link level cost of every one minute interval is available from an urban network, the developed predictive models can be adopted to predict the links cost, which includes travel time and/or GHG that are used to dynamically route vehicles to their destinations. Another essential component of this dissertation was the investigation of the best GHG costing approach, and it 
can become the reference for practitioners and researchers in the field. As every application is associated with specifications related to the level of aggregation of traffic, emissions, and required accuracy, the most suitable GHG costing approach will be defined for every unique case and is an added value to the literature.

To the best of our knowledge, this research is the first of its kind that developed and applied anticipatory multi-objective eco-routing strategies. This approach optimizes not only travel time, but also GHG emissions in a distributed routing framework, while adopting sophisticated predictive models. This research work provides the framework of anticipatory multi-objective eco-routing application and discusses the details of its process and components. The interactions between the components of a transport system, such as the traffic simulator, emission simulator, routing framework, vehicles, and intersections, are illustrated in a detailed manner. The strengths and weaknesses of different options are also demonstrated for future research work.

\subsection{Dissertation Outline}

The dissertation is composed of the following chapters. The framework followed in this dissertation is presented in Figure 1.1.

Chapter 2 presents a detailed review of the state-of-the-art and classifies the existing eco-routing models based on a multi-factor taxonomy. The three factors were the level of aggregation of the traffic and emission models, the scale of the case study, and the number of objectives optimized at a time. The strengths and weaknesses were discussed of every category. Finally, the potential of using the distributed routing system was illustrated with supporting results.

Chapter 3 included the background components, which will be used throughout this dissertation, and their description. First, the traffic model was presented in addition to the details of the adopted car following model. The emission model, MOtor Vehicle Emission 
Simulator (MOVES), was illustrated and its basic equations were discussed. Finally, a case study and the characteristics of its roads were presented.

Chapter 4 analyzes a framework of dynamic distributed routing, End-to-End Connected Automated Vehicles (E2ECAV) (Farooq and Djavadian, 2019) in the context of traffic and environmental aspects. A range of macro and micro indicators are considered for a comprehensive analysis.

Chapter 5 proposes myopic multi-objective eco-routing strategies and tests them in the E2ECAV routing framework. This framework overcame the shortcomings found in the studies from the related literature as summarized in Chapter 2. For the myopic multi-objective eco-routing application, microscopic level of aggregation for the traffic and emission models was applied in a large urban case study. At the same time, multiple objectives were optimized simultaneously. Results were analyzed at two levels: link and network.

Chapter 6 develops predictive models for GHG and speed based on the long short-term memory (LSTM) model, a deep learning approach. A comparison between LSTM and two other commonly used approaches, namely clustering and the autoregressive integrated moving average (ARIMA), has been conducted to define which model was the most suitable within the setting of this dissertation. The impact of utilizing a deeper LSTM network and systematically tuning the LSTM model hyper-parameters on the prediction performance was presented.

Chapter 7 employs the best predictive models from Chapter 6 and develops anticipatory multi-objective eco-routing strategies. A comprehensive comparison was made between the myopic routing strategies in Chapter 5 and the anticipatory routing strategies in this chapter. Results were analyzed at the link as well as network level.

Chapter 8 summarizes the major findings of this research and proposes ideas for future research on reducing produced GHG emissions by transportation systems. 


\section{Chapter 1: Introduction}

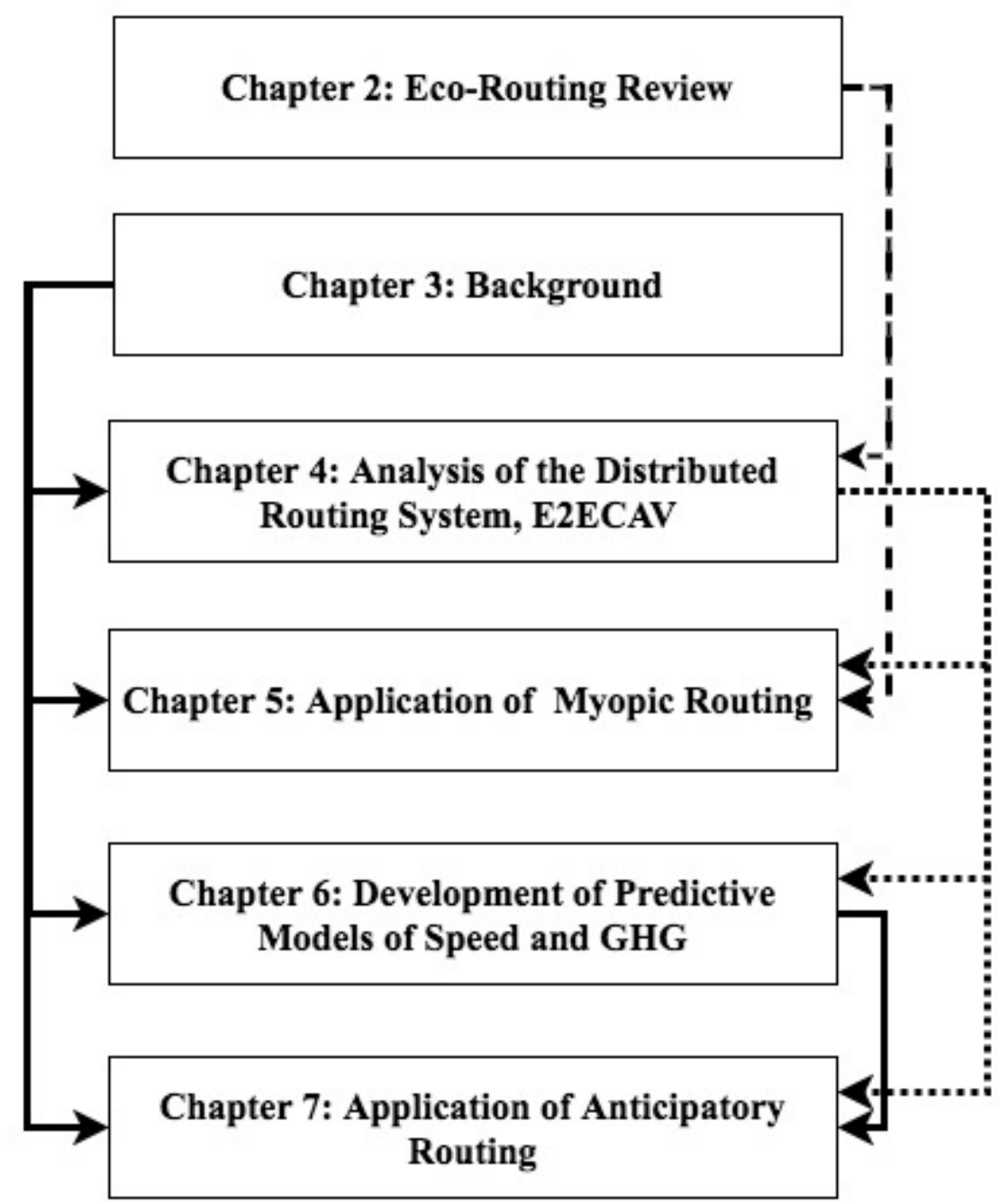

\section{Chapter 8: Conclusion and Future Remarks}

Figure 1.1 Dissertation Framework 


\section{Chapter Two}

\section{Review of Eco-Routing Studies}

This chapter has resulted in the following publication:

1. Alfaseeh, Lama, and Bilal Farooq (2020). "Multi-Factor Taxonomy of Eco-Routing Models and Future Outlook." Journal of Sensors. 


\section{$2.1 \quad$ Introduction}

Transportation systems have been consistently ranked as the largest source of GHG emissions in the U.S. (EPA, 2020b) and Canada (Environment and Climate Change Canada, 2020). GHG emission is one of the major factors contributing to climate change and global warming (Z. Liu et al., 2020). Among the suggested solutions to alleviate GHG produced by transportation systems, eco-routing, which considers the environmental aspect and is an extension of the routing concept, is a popular and effective approach. Nitrogen Oxide (NOx) is among the pollutants produced by transportation systems and has a negative impact on the environment and public health. NOx emissions lead to the formation of tropospheric $\mathrm{O}_{3}$, which is also a greenhouse gas. Furthermore, NOx is associated with several adverse effects on the public health with respect to acid deposition and drinking water nitrate. (Price et al., 1997). To improve the performance of transportation systems, it is beneficial to also consider NOx as a routing objective and/or performance indicator.

Initially, routing was based on one routing objective, which is travel time (Tzeng and C.-H. Chen, 1993). However, in the last few decades, researchers started incorporating more than one objective, while routing vehicles to their destinations. Multiple objective optimization is associated with a high level of complexity due to the heterogeneous interaction between the different objectives that might be competing. For example, an improvement in the network throughput may have an adverse impact on the environmental aspect. A longer travelling distance may contribute to a lower travel time, but it may also result in more GHG emissions (Alfaseeh and Farooq, 2020a).

In this chapter, literature related to the fundamental elements is discussed, such as routing, eco-routing, traffic assignment and their relationship with routing in general. A three factor taxonomy was developed based on the most representative characteristics of the eco-

routing studies. The strengths and weaknesses of each category of the taxonomy are discussed. Finally, concluding remarks are included. Table 2.1 illustrates the acronyms and 
abbreviations used in this chapter. The findings of this chapter trigger the need for further research that is addressed throughout this dissertation.

This chapter is organized as follows. Section 2.2 discusses the concepts of routing, ecorouting, and traffic assignment. In addition, the relationship between traffic assignment and routing is demonstrated. Section 2.3 presents the main vehicle emission models and their sub models with examples. The proposed taxonomy of the eco-routing studies is illustrated in Section 2.4. In Section 2.5, the eco-routing studies are classified based on the taxonomy presented in the previous section. Finally, Section 2.6 summarizes the findings in this chapter.

\subsection{Vehicle Routing, Eco-Routing, and Traffic Assign- ment}

Routing is guiding vehicles to their destinations and is mainly based on a single objective, such as travel time, distance, emissions, or fuel. Generally, routing was applied to minimize travel time from origins to destinations (Cherkassky, Goldberg, and Radzik, 1996). To apply routing, algorithms are required to define the optimal routes from origins to destinations. It was found in literature (Cherkassky, Goldberg, and Radzik, 1996) that the Dijkstra, Bellman-Ford, A*, Floyd-Warshall, Johnson, and Viterbi routing algorithms were the most important. The Dijkstra algorithm is a very popular routing algorithm and solves the singlesource shortest path problem with non-negative edge weights. It was proposed in 1956 but was published three years later. The Bellman-Ford algorithm solves the single-source problem if edge (links) weights may be negative, which occurs when incentives are provided for choosing certain edges. The Bellman-Ford algorithm was first proposed by Alfonso Shimbel (1955), but it is instead named after Richard Bellman and Lester Ford Jr., who published it in 1958 and 1956, respectively. The A* search algorithm finds the shortest 
Table 2.1 Acronyms and Abbreviations Used in Chapter Two

\begin{tabular}{|c|c|}
\hline $\begin{array}{l}\text { Acronyms and } \\
\text { abbreviations }\end{array}$ & Full name \\
\hline STA & Static traffic assignment \\
\hline DTA & Dynamic traffic assignment \\
\hline $\mathrm{UE}$ & User Equilibrium \\
\hline SO & System Optimal \\
\hline$W_{t}, W_{d}, W_{e}$, and $W_{f}$ & Weights of travel time, distance, emissions, and fuel, respectively \\
\hline$T_{i}, D_{i}, E_{i}$, and $F_{i}$ & Travel time, distance, emissions, and fuel of link $i$, respectively \\
\hline IS1 & $\begin{array}{l}\text { Model adopting microscopic traffic and emission models, small case study, } \\
\text { and optimizing one routing objective }\end{array}$ \\
\hline IL1 & $\begin{array}{l}\text { Model adopting microscopic traffic and emission models, large case study, } \\
\text { and optimizing one routing objective }\end{array}$ \\
\hline AS2 & $\begin{array}{l}\text { Model adopting macroscopic traffic and emission models, small case study, } \\
\text { and optimizing more than one routing objective }\end{array}$ \\
\hline AL1 & $\begin{array}{l}\text { Model adopting macroscopic traffic and emission models, large case study, } \\
\text { and optimizing one routing objective }\end{array}$ \\
\hline AL2 & $\begin{array}{l}\text { Model adopting macroscopic traffic and emission models, large case study, } \\
\text { and optimizing more than one routing objective }\end{array}$ \\
\hline MS2 & $\begin{array}{l}\text { Model adopting different levels of aggregation of traffic and emission models, } \\
\text { small case study, and optimizing more than one routing objective }\end{array}$ \\
\hline ML1 & $\begin{array}{l}\text { Model adopting different levels of aggregation of traffic and emission models, } \\
\text { large case study, and optimizing one routing objective }\end{array}$ \\
\hline
\end{tabular}

path for a single origin-destination pair using heuristics to try to speed up the search. The Floyd-Warshall algorithm solves the shortest paths for all pairs. The Johnson algorithm also solves the shortest paths for all pairs but may be faster than the Floyd-Warshall algorithm on sparse graphs. The Viterbi algorithm solves the shortest stochastic path problem with 
an additional probabilistic weight on each node (Cherkassky, Goldberg, and Radzik, 1996).

The routing concept has been used by public agencies for decades and their main tool is the road variable message sign (VMS) to direct traffic through the best routes. However, due to the emergence of ICT, the availability of route guidance services by private parties have skyrocketed, and personal navigation platforms have been growing due to their increased affordability and introduction by commercialization (Chiu et al., 2011). Recently, environmental indicators have been considered and eco-routing has substituted the conventional routing concept that only considers the traffic variables. Other synonym terms of eco-routing can be pollution routing (Bektaş and Laporte, 2011) or green-routing (Guo, S. Huang, and Sadek, 2013).

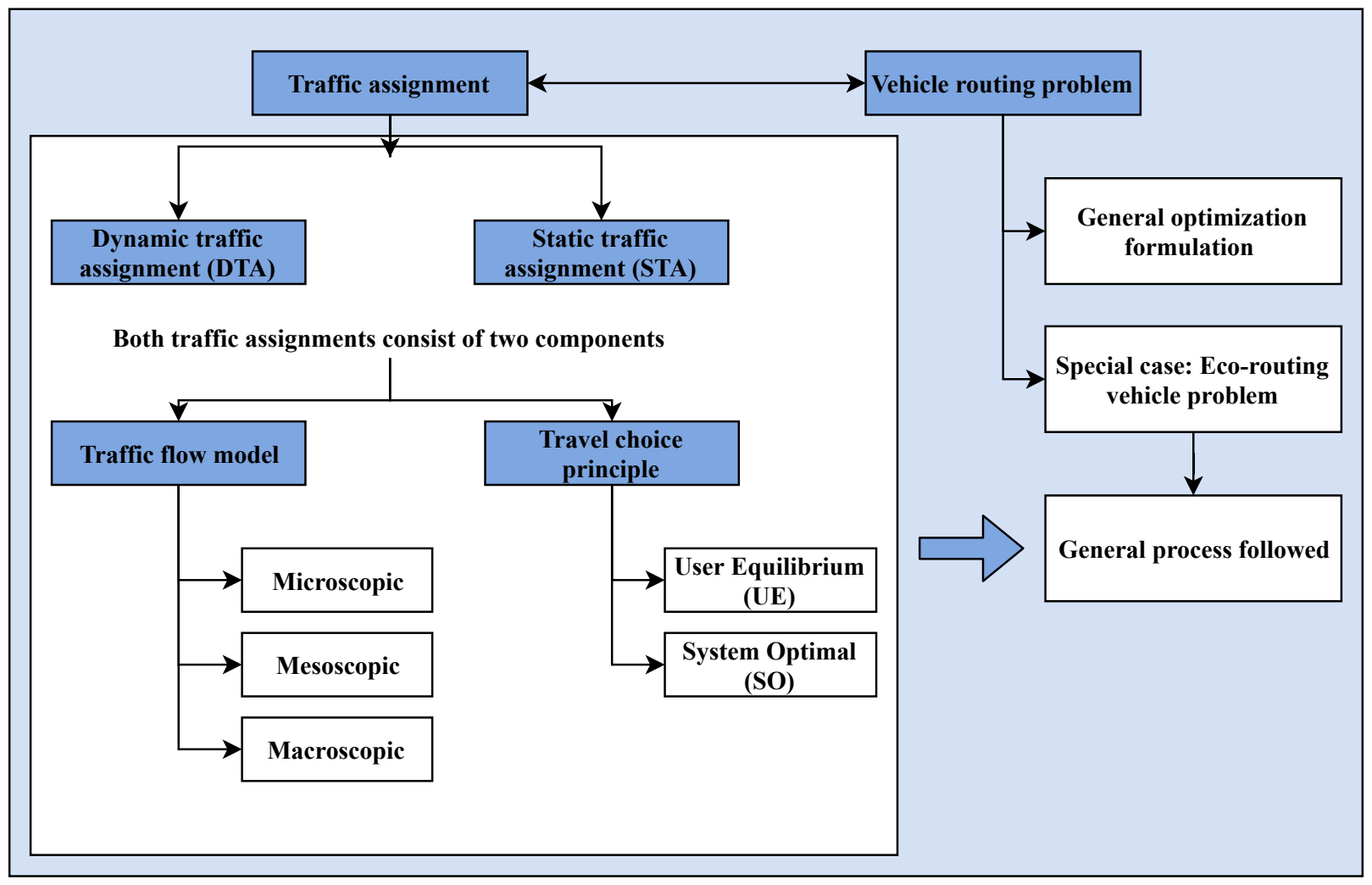

Figure 2.1 Traffic Assignment Classification, Components, and Traffic Assignment Relationship with Routing (Alfaseeh and Farooq, 2020b)

In general, to route vehicles based on a routing objective, the link cost is required to 
estimate the cost of possible paths and to consequently choose the optimal route. The cost is obtained from historical data, data collected from sensors, probe vehicles, or traffic assignment. Traffic assignment focuses on the choice of a path from an origin to a destination (Papacostas and Prevedouros, 1993). When a path is chosen, which is based on the objectives set by drivers or by governments, any traffic or environmental variable is estimated accordingly. Traffic assignment is the fourth step in the conventional transportation forecasting model and comes after trip generation, trip distribution, and mode choice. It is considered as the equilibration model between the travel demand and transportation system supply (Papacostas and Prevedouros, 1993). Traffic assignment is a common tool adopted to define the link cost (Papacostas and Prevedouros, 1993). Figure 2.1 includes the types of the traffic assignment models, their components, and the relationship between traffic assignment and routing. Traffic assignment is classified into two categories: static and dynamic. The static traffic assignment (STA) considers equal in and out flow of a studied link. This means that the congestion phenomenon is not captured efficiently. The main outcomes of the STA models include average speed, traffic volume, level of service, and traffic composition (Chiu et al., 2011), and they are employed to estimate the weights of the traffic characteristics to define the optimal routes (Szeto, Jaber, and Wong, 2012). On the other hand, the dynamic traffic assignment (DTA) represents the congestion more realistically by examining the changes in the traffic conditions over time. Thus, the weights obtained based on DTA are more reliable for routing compared to STA (Y. Wang et al., 2018). Both traffic assignment models consist of two components, the traffic flow model and the travel choice principle. The traffic flow model can be either microscopic, mesoscopic, or macroscopic (Luo et al., 2016). The microscopic traffic flow model captures the dynamics of every vehicle while adopting a high level of a spatial and temporal resolution. The outputs include the speed, position, and acceleration of every vehicle at every time step. The macroscopic model is associated with a lower level of resolution compared to microscopic models since it requires aggregated information about the vehicular dynamics (Szeto, Jaber, and Wong, 2012). Finally, the 
mesoscopic flow model goes between the microscopic and macroscopic models. It captures the vehicular flow at an aggregated level, while the behaviour rules are taken in detail. The dynamics between the vehicles are applied at a microscopic level, and vehicles of the same characteristics are grouped in small packets. The trajectories of these packets are applied following the logic of the microscopic traffic flow model. The macroscopic flow-density relationship is employed to model the flow propagation between links as in the macroscopic models.

With regards to the travel choice models, Wardrop defined the two principles: the User Equilibrium (UE) and the System Optimal (SO) (Zuurbier, 2010). UE is when "the journey cost on all the routes actually used are equal and less than those which would be experienced by a single vehicle on any unused route" (Zuurbier, 2010). This means that drivers look for the route with the least cost regardless of the impact at the network level. The UE method is the most widely used trip assignment method in transportation. In the case of UE, a driver cannot unilaterally minimize his travel time by switching to another route. Under this condition, the travel time is the same on each used path between any origin destination (OD) pair. Nevertheless, the total system travel time in the case of UE traffic assignment may not be the minimum (Szeto, Jaber, and Wong, 2012). On the other hand, the SO is when "the average journey cost is a minimum for all routes in a network" (Zuurbier, 2010). The minimum of the total system travel time can be found under this condition. The SO assignment is what authorities aim to achieve.

The general optimization process of routing, whether it considers traffic and/or environmental variables, follows Equation 2.1. When environmental variables are part of the optimization process, routing is replaced with eco-routing.

$$
\min \left\{\sum_{i=1}^{n} W_{t} \cdot T_{i}+\sum_{i=1}^{n} W_{d} \cdot D_{i}+\sum_{i=1}^{n} W_{e} \cdot E_{i}+\sum_{i=1}^{n} W_{f} \cdot F_{i}\right\}
$$

Where $T_{i}$ is travel time on link $i ; D_{i}$ is distance traversed on link $i$; $E_{i}$ is emissions on link $i$ that can include $\mathrm{CO}_{2}, \mathrm{CO}, \mathrm{NOx}$, or any other pollutant; $F_{i}$ is fuel consumed on link 
$i$; $n$ is number of links of a path $k$; and $W_{t}, W_{d}, W_{e}$, and $W_{f}$ are weights associated with travel time, distance, emissions, and fuel, respectively. The variables are obtained based on a defined aggregation level, which can be microscopic, mesoscopic, or macroscopic. The level of aggregation impacts explicitly the quality of estimation, which has a direct effect on the routing performance. To better understand the application of the general optimization process, the variables of a specific road link $i$ in downtown Toronto are extracted from one of the simulation runs. As found from the simulation data points, while adopting a minute updating interval, the speed of that specific link varies from 10 to $80 \mathrm{~km} /$ hour throughout the simulation. The link $i$ length is $0.5 \mathrm{~km}$, which is represented by $D_{i}$ in Equation 2.1 . The travel time, which reflects on $T_{i}$, varies from 0.0026 to 0.02 hour based on the speed at every minute. If the cost of emissions, $E_{i}$, in Equation 2.1 is taken in a form of emission rate (ER), the value ranges from 5137 to $14900 \mathrm{~g} /$ hour. The unit adopted for emissions can be $\mathrm{kg} / \mathrm{h}$ or even ton/h. Finally, the amount of consumed fuel can be estimated based on the distance travelled (Natural Resources Canada, 2020). The conversion factor from distance to fuel is subject to the traffic condition and vehicle characteristics. For example, the conversion factor would be 0.078 litre/ $\mathrm{km}$ for a gasoline-fuelled Honda Civic sedan with a four-cylinder engine and 2 litre engine size on an urban road (Natural Resources Canada, 2020). Since the link $i$ length is $0.5 \mathrm{~km}$, the fuel consumed by the Honda Civic would be around 0.0395 litres (Natural Resources Canada, 2020). By examining the values of the four possible variables based on only one link, the dramatic difference in their scales is illustrated. If more than one objective is optimized simultaneously, the one with the larger scale would dominate the optimization process. For instance, optimizing $\mathrm{T}$ and $\mathrm{E}$ in this case means that $\mathrm{E}$ is the dominating variable in the optimization process. This brings in the need for weights to account for the different units of the optimized variables $(T, D, E$, and $F)$ in Equation 2.1. Converting the different values to a monetary value is common in practice for routing optimization problems (Aziz and Ukkusuri, 2012). Linear optimization has been considered as it is suitable for minimization and maximization problems (Gass, 2003). 
Figure 2.2 demonstrates the general process followed while applying eco-routing. The variables in concern are obtained from either sensors (real data) or from traffic flow models. Generally, the used emission model and its level of aggregation define the required traffic variables. Speed, acceleration, deceleration, and distance are the most important traffic indicators when it comes to emission estimation. It is worth mentioning that the aggregation level compatibility of the traffic and emission models plays a crucial role in improving the eco-routing performance. When a microscopic level of aggregation is used, the traffic and environmental states are represented more accurately. When a microscopic emission model is used, as will be presented below in Section 2.3, a microscopic traffic model should also be applied.

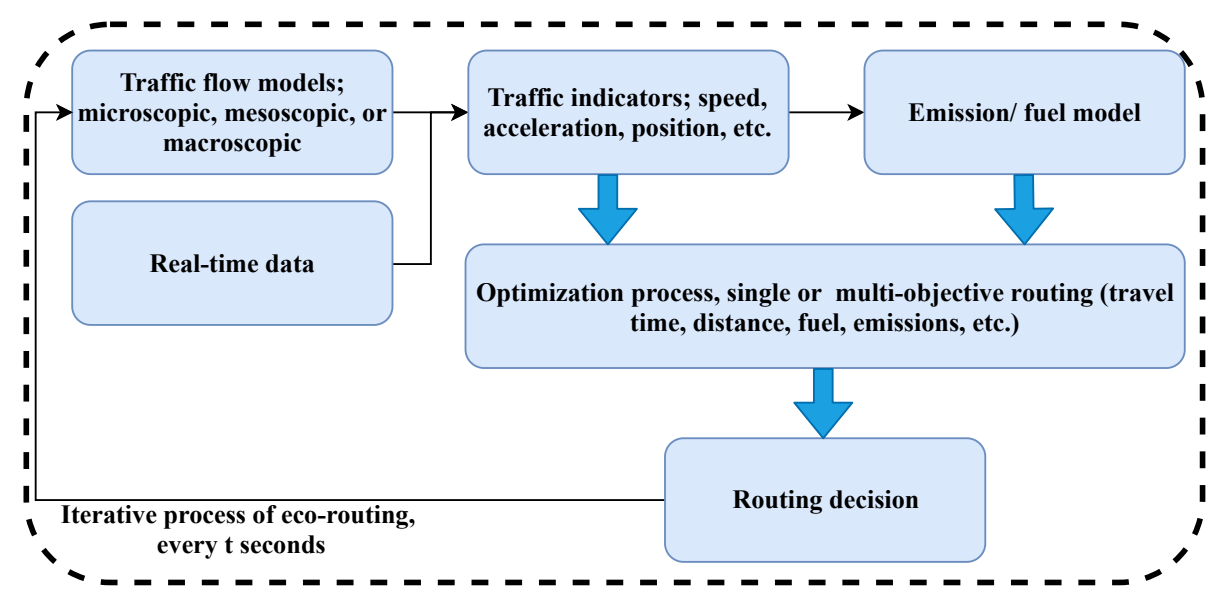

Figure 2.2 Eco-Routing Framework (Alfaseeh and Farooq, 2020b)

The required traffic variables are the inputs of the emission model to estimate the environmental variables needed for the eco-routing, such as GHG. Based on the routing objective, the traffic and/or environmental indicators are utilized in the general optimization formulation, as shown in Equation 2.1, to estimate the cost of paths and specify the optimal route of the least cost. Once the route is chosen, the traffic characteristics are updated accordingly. It is essential to note that this is an iterative process for every $t$ seconds (Y. Wang et al., 2018). Regardless of the level of aggregation, the link cost is updated based on the 
traffic and environmental indicators for every iteration.

\subsection{Vehicle Emission Modelling}

To better assess the impact of transportation systems on the environment, emission models have been developed. Emission models estimate the emissions produced by multiplying a defined emission factor expressing the mass of emissions produced per distance unit, time unit, or burnt fuel unit by the corresponding vehicle activity data, such as vehicle kilometres travelled (VKT), vehicle mile travelled (VMT), time spent in a specific driving condition, or fuel consumed. Over the last few decades, the emission models have become more sophisticated by incorporating driving behaviours while considering a higher level of resolution and a higher level of details from the input data (Y. Wang et al., 2018). The emission models are classified based on two factors: the level of spatial and temporal aggregation and the driver behaviour aggregation. To connect emission to congestion explicitly and comparably to traffic models, static and dynamic categories are adopted (Y. Wang et al., 2018).

Figure 2.3 illustrates the aforementioned two main categories of the emission models and their sub categories.

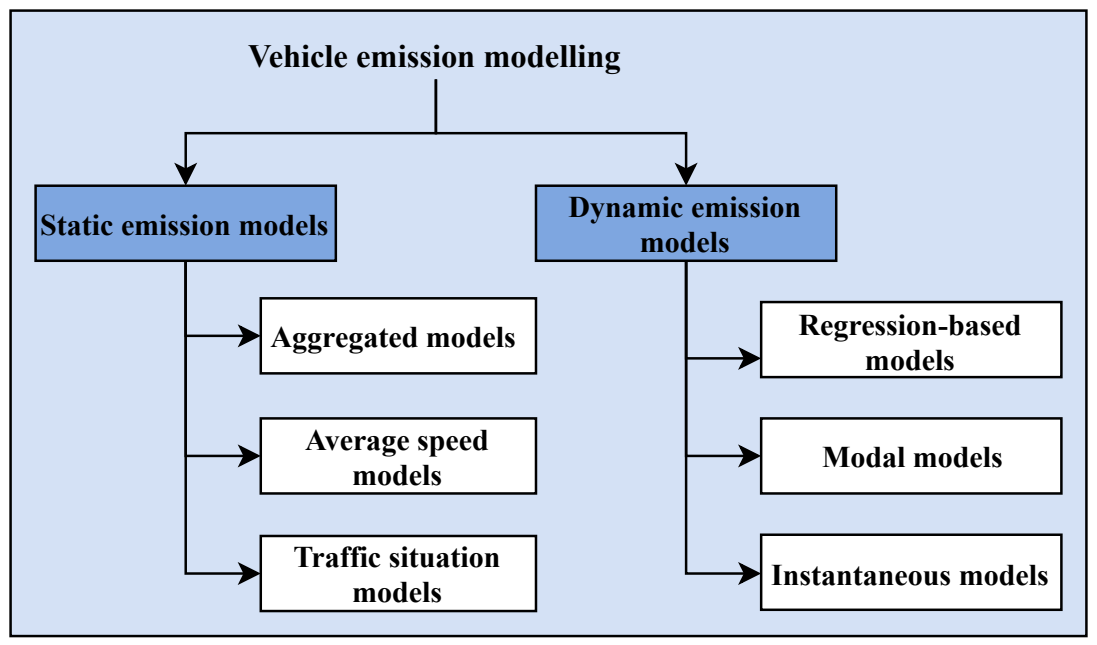

Figure 2.3 Classification of Vehicle Emission Models (Alfaseeh and Farooq, 2020b) 


\subsubsection{Static Emission Models}

This type of emission model is based on the macroscopic level of aggregation of information. For this type of emission model, national statistical (e.g. public road and street mileage), field measurement (e.g. traffic flow data obtained from cameras or sensors), or forecasted traffic flow from STA models are employed when the emissions are estimated at a low level of resolution, which is suitable for transportation planning purposes. Despite the drawbacks associated with this type, it is still very commonly used due to its simplicity and ease of use (Szeto, Jaber, and Wong, 2012). The static emission models are further classified based on the level of complexity as follows. 1) Aggregated models are associated with an extremely low level of resolution for the used data. These models estimate emissions based on the emission factors of the vehicle kilometres travelled (VKT) or the fuel consumed (Szeto, Jaber, and Wong, 2012). Inventory and the Energy Book for transport are two examples of the aforementioned models (Szeto, Jaber, and Wong, 2012). 2) Average-speed models estimate emissions based on the average speed on the links and their corresponding emission factors. The emission factors are g/VKT (Ntziachristos et al., 2000). An example includes the Computer Program to Calculate Emissions from Road Transport (COPERT) (Ntziachristos et al., 2000). Other examples are a computer program that estimates different emission factors (MOBILE) (US Environmental Protection Agency, 2003) and the EMFAC2014 (California Air Quality Research Board, 2014). It is essential to note that this type of model does not capture the congestion on the links efficiently. Lastly, 3) traffic situation models estimate emissions based on traffic conditions and emission factors associated with each traffic condition (INFRAS, 2004). Examples of this type include the Handbook Emission Factors for Road Transport (HBEFA) (INFRAS, 2004) and the Assessment and Reliability of Transport Emission Models and Inventory Systems (ARTEMIS) (Boulter et al., 2007). The last model is the most detailed compared to the other two static emission models (Y. Wang et al., 2018). 


\subsubsection{Dynamic Emission Models}

Unlike the static emission models, dynamic emission models are based on the second-bysecond vehicular dynamics. They take into account the related traffic inputs and simultaneously estimate the emissions of a vehicle based on operational conditions at a high level of resolution. Compared to static models, dynamic emission models are able to capture changes more realistically by reflecting on existing situation. As a result, this type of model is more suitable for routing applications. Dynamic emission models are generally classified into three categories based on the aggregation level of data points. 1) Regression-based models estimate the emissions based on regression approaches and variables that represent the driving cycles. Speed, acceleration levels, or speed-time profile data is required to define the emission factor ( $\mathrm{g} / \mathrm{sec}$ or $\mathrm{g} / \mathrm{VKT})$. Microscopic data is suitable for this type of model, and this model can be used aside with the DTA. However, regression-based models are very sensitive to overfitting the calibrated data. Examples include VERSIT+ (Smit, Smokers, and Rabé, 2007) and the Virginia Tech Microscopic Energy and Emission Model (VT-Micro) (Rakha, Ahn, and Trani, 2003). 2) Modal models estimate the emissions based on the emission factors of each driving mode, such as start, idle, acceleration, deceleration, and cruise. The vehicle and the cycle characteristics are required as inputs. An example of this type is the Mobile Emissions Assessment System for Urban and Regional Evaluation (MEASURE) (Fomunung, Washington, and Guensler, 1999). Finally, 3) instantaneous models are generally the most detailed emission models. Both dynamic operating variables (e.g., second-by-second speed, road grade, and vehicle accessory use) and static mode parameters (to characterize the vehicle tailpipe emissions for the appropriate vehicle/technology category) are required as inputs. However, this type of model is difficult to calibrate and requires intensive vehicular characteristics, operations, and locations. Examples include Passenger car and Heavy-duty Emission Model (PHEM) (Hausberger et al., 2003), Comprehensive Modal Emission Model (CMEM 2) (Scora and M. Barth, 2006), and MOtor Vehicle Emission Simulator (MOVES 
3) (United States Environmental Protection Agency, 2014).

\subsection{Taxonomy of the Eco-Routing Studies}

To classify the existing literature, a three-factor taxonomy was proposed as illustrated in Figure 2.4. The first factor reflected on the employed level of aggregation of the traffic and emission models. Basically, the purpose of the traffic models is to obtain the average or instantaneous traffic variables to estimate the emissions. For this factor, four categories were taken into account as shown below.

- Microscopic (I) is associated with a high level of spatial and temporal resolution while estimating the traffic and environmental indicators. However, this comes at the cost of computational time, resources, and input data requirements.

- Macroscopic $(\boldsymbol{A})$ is associated with a course level of spatial and temporal resolution. Hence, this category is associated with less accuracy compared to the I Models. However, $\boldsymbol{A}$ Models are simply used as a traffic flow model when high-resolution data points are not available.

- Mesoscopic $(\boldsymbol{E})$ is in between the microscopic and macroscopic flow models in terms of the aggregation level.

- Mixed aggregation ( $M)$ includes models of different levels of aggregation for the traffic and emission models.

The second factor of the proposed taxonomy reflected on the scalability of the model. A larger scale generally results in more realistic outcomes. However, this comes at the cost of computation and complexity. There two categories:

- A large case study $(\boldsymbol{L})$ can be an entire metropolitan area or central zones of a region. 


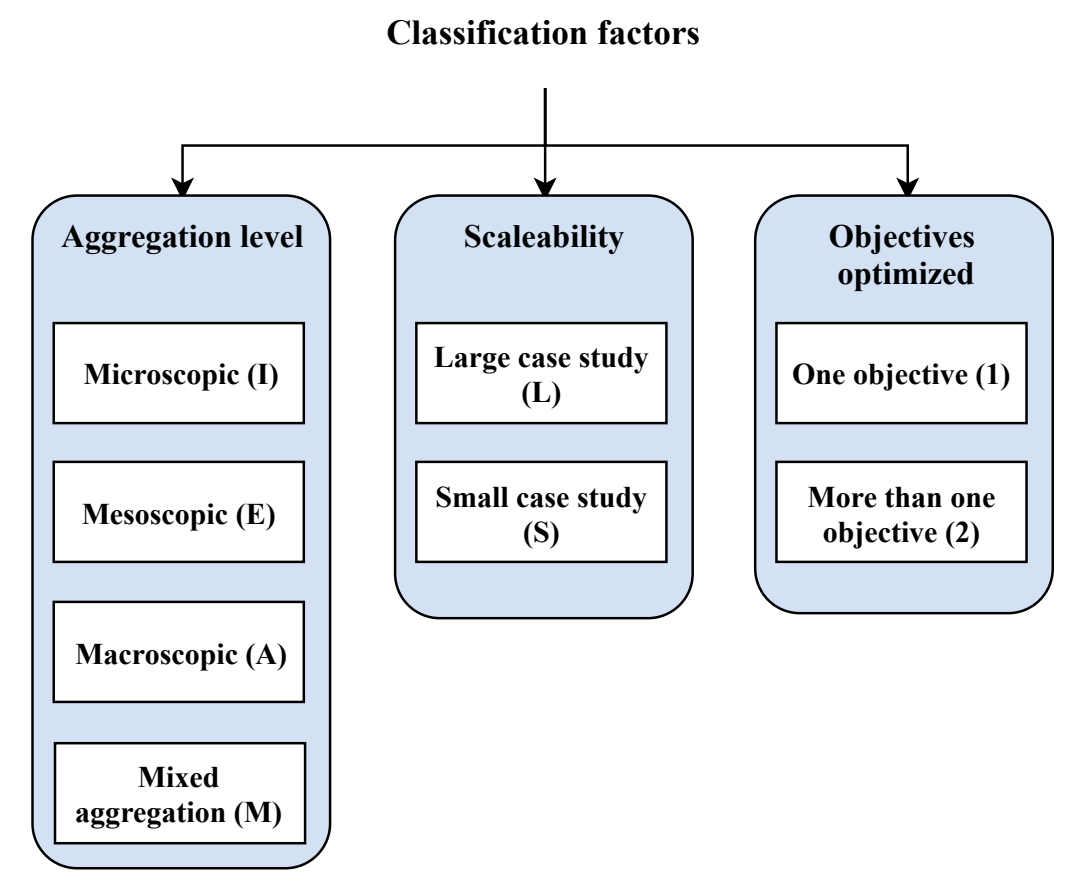

Figure 2.4 Abbreviations of the Proposed Three Factor Taxonomy (Alfaseeh and Farooq, 2020b)

- A small case study (S) can be a segment of a road, a well-defined part of a highway, or a zone with limited number of intersections.

Finally, the third factor of taxonomy was related to the number of optimized objectives. From earlier studies, a single objective was taken because contradictions could develop between routing objectives. While improving one objective, an adverse impact may be generated with another objective. Nevertheless, over time, environmental objectives have been taken into account, in addition to other objectives, to provide better performing transportation systems from an environmental point of view. Two main categories have been considered for the third factor of taxonomy:

- Single objective (1) considers only one routing objective, such as travel time, distance, GHG, or NOx.

- More than one objective (2) optimizes more than one routing objective. Different 
combinations can be chosen while routing vehicles to their destinations. For multiobjective routing, travel time is generally prioritized and combined with one or more other objectives, such as GHG, NOx, distance, or fuel.

As an example of the classification factors, $\boldsymbol{A} \boldsymbol{L} \mathscr{2}$ is for eco-routing models that employ macroscopic traffic flow and emission/fuel models with a large case study and optimized more than one objective at a time.

\subsection{Classification of Existing Literature}

Table 2.2 demonstrates the classified eco-routing studies based on the three factors proposed in Section 2.4. The details of the reviewed studies are presented below following the order of the first factor of the proposed taxonomy.

\subsubsection{Microscopic (I) Models}

The main characteristic of $\boldsymbol{I}$ Models is the employed high spatial and temporal resolution of data as the microscopic level of aggregation is adopted to estimate traffic and emissions indicators.

Rakha, Ahn, and Moran (2012) developed two eco-routing algorithms. The first algorithm was based on vehicle sub-populations (ECO-Subpopulation Feedback or ECO-SFA), and the

other algorithm was based on individual agents (ECO-Agent Feedback Assignment or ECOAFA). The model belongs to the $\boldsymbol{I S 1}$ classification as shown in Table 2.2. The main objective function was to minimize either fuel consumption or travel time, and this means that only one objective in Equation 2.1 was optimized at a time. The main difference between the ECO-SFA and ECO-AFA was that the former gave periodically updated routes for every vehicular class. On the other hand, the latter gave routes for every vehicle based on request. Routing was multi-class because vehicles can only use information from vehicles of the same 
class. Routing was also stochastic as it accounts for the uncertainty with the cost of the links (Rakha, Ahn, and Moran, 2012).

Ahn and Rakha (2013) applied the same logic followed in Rakha, Ahn, and Moran (2012) and investigated the impact of applying a dynamic eco-routing system that took into account different market penetration rates (MPRs) and congestion levels. The eco-routing method goes under the $\mathbf{I L} \mathbf{1}$ classification as demonstrated in Table 2.2, and it allowed for a multiclass, stochastic, and dynamic eco-routing system. Routes were updated based on real data from vehicles in the network. Vehicles shared information related to their fuel consumption with a Traffic Management Center (TMC) via roadside units (RSUs). The TMC processed fuel consumption information from vehicles in the network and provided vehicles with optimal routes (Ahn and Rakha, 2013).

J. Sun and H. X. Liu (2015) optimized one objective at a time in a signalized traffic network. Their approach, which is classified as $\mathbf{I S 1}$ as shown in Table 2.2, was based on applying the Markov decision process (MDP) that was converted to a linear programming formulation. This study used high resolution (microscopic) data (vehicle arrival and signal status) instead of GPS data. Vehicle trajectories were estimated based on a proposed method that depended on the conditions of the traffic signal status and the number of vehicles arriving at intersections. For every second, trajectories of vehicles were fed into a microscopic emission model. The study optimized one objective at a time in Equation 2.1 ( $T$ or E). A comparison was made between the shortest and minimum emissions paths. A reduction in emissions was associated with the latter routing scenario. J. Sun and H. X. Liu (2015) extended the concept of optimizing environmental indicators by constraining them. $T$ was set as the main objective, while $E$ was constrained.

Elbery, Rakha, Elnainay, et al. (2015) moved towards utilizing one form of the ICT improvements, where the vehicle to infrastructure (V2I) communication was adopted. As shown in Table 2.2, the proposed eco-routing model is classified as $\mathbf{I S 1}$. The model was based on integrating two models: OPNET and INTEGRATION. OPNET was the model 
that handled the communication between the vehicles and TMC. When vehicles traversed certain links, INTEGRATION calculated information about their experiences in terms of emissions, travel time, and location, and the information was sent to the TMC. TMC defined optimal eco-routes and sent them to vehicles when requested. The study investigated the impact of errors due to data packet drops and delays, and it was found that the impact was not significant. Furthermore, Elbery, Rakha, Elnainay, et al. (2015) found that the proposed model was robust against delays and data packet drops (Elbery, Rakha, Elnainay, et al., 2015).

Elbery, Rakha, ElNainay, et al. (2016) proposed an ant-colony-based approach, which was based on a subpopulation feedback eco-routing (SPF-ECO) algorithm that was similar to Rakha, Ahn, and Moran (2012) and Ahn and Rakha (2013). The proposed eco-routing approach goes under $\boldsymbol{I S 1}$ classification as shown in Table 2.2. The authors mainly tried to overcome the main shortcoming of the SPF-ECO approach, which was the delayed updates. The main enhancement of the ant colony eco-routing (ACO-ECO) was the more realistic representation of blocked and unoccupied links within a network. Furthermore, it considered periodical updates that helped to obtain optimal routes. The model was applied in a centralized routing system framework in which vehicles sent the traversed cost of links to TMC. A comparison between ACO-ECO and SPE-ECO was conducted. In Equation 2.1, $F$ was only optimized, and the optimization contributed to less emissions $E$. With regards to results, ACO-ECO performed similarly to the SPE-ECO when the operation was normal (links were unblocked and occupied). Yet, when links were blocked or unoccupied, ACOECO reduced fuel consumption, travel time, and emissions compared to SFE-ECO (Elbery, Rakha, ElNainay, et al., 2016).

Bandeira et al. (2018) assessed the impact of different eco-routing strategies in terms of system travel time and other environmental criteria. The second-by-second data of the vehicles in the transport system were obtained from Verkehr In Städten - SIMulationsmodell (VISSIM). The distribution of vehicle-specific power (VSP) modes was calculated. Finally, 
emissions on a road segment were estimated based on the amount of time every vehicle spent in each VSP mode multiplied by the proper emission factor. The proposed eco-routing model belongs to the $\boldsymbol{I L 1}$ classification. Following the equations in Bandeira et al. (2018), the time spent and corresponding total emissions produced by a pollutant ( $\mathrm{NOx}, \mathrm{CO}, \mathrm{HC}$, and $\mathrm{CO}_{2}$ ) were estimated for every mode. Weights were given to the pollutants that were taken into account. Then, environmental damage (ED) costs were obtained based on the weights. While referring to Equation 2.1, one objective was optimized at a time while routing vehicles. Routing depended on the travel time cost for non-eco-routed vehicles, while emissions, environmental damage, and pollutant costs were determined for eco-routing vehicles. Two scenarios were considered in terms of the system demand and MPR of the ecorouting vehicles. With regards to the major results, minimizing emissions of the individuals while routing may lead to more emissions overall compared to a standard UE assignment. Nevertheless, when demand was moderate and MPR of eco-routing vehicles was $100 \%, \mathrm{CO}_{2}$ and NOx emissions in the system were reduced by $9 \%$ compared to the standard UE travel time case. Furthermore, the total system emissions were reduced at the cost of a slight increase by up to $5 \%$ in travel time (Bandeira et al., 2018).

A recent study by Elbery and Rakha (2019), which belongs to the $\boldsymbol{I L} \mathbf{1}$ classification, elaborated on the logic followed in Elbery, Rakha, Elnainay, et al. (2015). The proposed eco-routing model depended on two main features: 1) the existence of connected vehicles that can communicate with a TMC and 2) the existence of sensor vehicles or probe vehicles that were uniformly distributed in the studied network. The main goal of the study was to examine the efficiency of the communication feature proposed under different scenarios. The model suggested that probe vehicles were distributed to collect information, in particular the consumed fuel, on traversed links. This means that $F$ variable of the optimization formulation in Equation 2.1 was optimized. The obtained information was then sent to TMC that updated routes based on real-time data. Connected vehicles requested optimal eco-routes from TMC that did the related calculations. Different MPRs of probe vehicles 
and different demand levels were considered, and their impact on the model performance was assessed. Furthermore, packet drops and delays may occur in reality, and the impact of packet drops and delays were investigated with a case that was based on a "realistic communication" system. The major findings of the study were the following: 1) for the ideal communication condition (no packet drops nor delay), greater improvements to the networkwide fuel consumption levels were offered with higher MPRs; 2) the system required $20 \%$ to $30 \%$ of probe vehicles to produce acceptable results in the case of ideal and realistic communication conditions; 3) for a realistic communication condition (with packet drops and delays), the higher MPR caused more emissions due to errors in routing vehicles that were triggered by the higher frequency of packet drops; and 4) the packet drops and delays of the VANET communication network had a significant impact on the performance of a dynamic routing system (Elbery and Rakha, 2019).

\subsubsection{Mesoscopic (E) Models}

From the extensive literature review, it was concluded that none of the studies applied a mesoscopic level of model aggregation to both traffic and emission models. This could have occurred since a great amount of time and computational power are required when a mesoscopic traffic flow model is used to simulate the traffic flow, despite the reduced level of resolution compared to the microscopic level of aggregation (Y. Xu et al., 2014).

\subsubsection{Macroscopic (A) Models}

The main characteristic of this category is the low level of spatial and temporal resolution. Thus, this category is associated with less accuracy compared to I Models. However, this type of model is used when high-resolution data points are not available.

Tzeng and Chen Tzeng and C.-H. Chen (1993) incorporated the environmental aspect while assigning vehicles to their destinations in a model, which goes under the $\boldsymbol{A} \boldsymbol{L} \mathscr{2}$ classi- 
fication as shown in Table 2.2. Nonlinear programming techniques were used to find the optimal solution. Time and emissions were estimated based on the Bureau of Public Roads (BPR) function. When multi-objective routing was applied, the approach was based on weights to normalize the optimized variables. Sets of weights were compared to choose the optimal weight for every variable. While referring to Equation 2.1, weights were applied for T, D, and $E$ (Tzeng and C.-H. Chen, 1993).

A study by Benedek and Rilett (1998), which belongs to the $\boldsymbol{A L} \mathbf{1}$ classification, considered both UE and SO traffic assignments. With reference to Equation 2.1, one variable was optimized at a time. This study dealt with the static traffic assignment case when it came to including the emission aspect (Benedek and Rilett, 1998).

A study by Luo et al. (2016), which belongs to the $\boldsymbol{A} \boldsymbol{S} \mathcal{2}$ classification, proposed a framework that was based on advanced controlling technology, which was called Model Predictive Control (MPC). An online optimization was applied and the future state was predicted based on the current state. In terms of the optimization formulation in Equation 2.1, the study used weights for $T, E$, and $F$. The MPC was known to be an efficient tool for multiple control objectives and constraints (Luo et al., 2016). Moreover, Luo et al. (2016) stated that MPC is a robust tool when it comes to model mismatch, system uncertainties, and disturbances (Luo et al., 2016). A real-time en-route diversion control strategy with multiple objectives was designed in an MPC framework. VMS was used at the point where vehicles would have information about the best route that satisfied the set of objectives. The variables were optimized one at a time, and then, a multi-objective scenario was applied that considered $T, E$, and $F$ in Equation 2.1. During the peak hour, the multi-objective routing strategy showed a clear improvement for the three variables. Furthermore, for the off-peak case, the same scenario showed a slight increase in total travel time but showed a reduction in both total emissions and consumed fuel (Luo et al., 2016). 


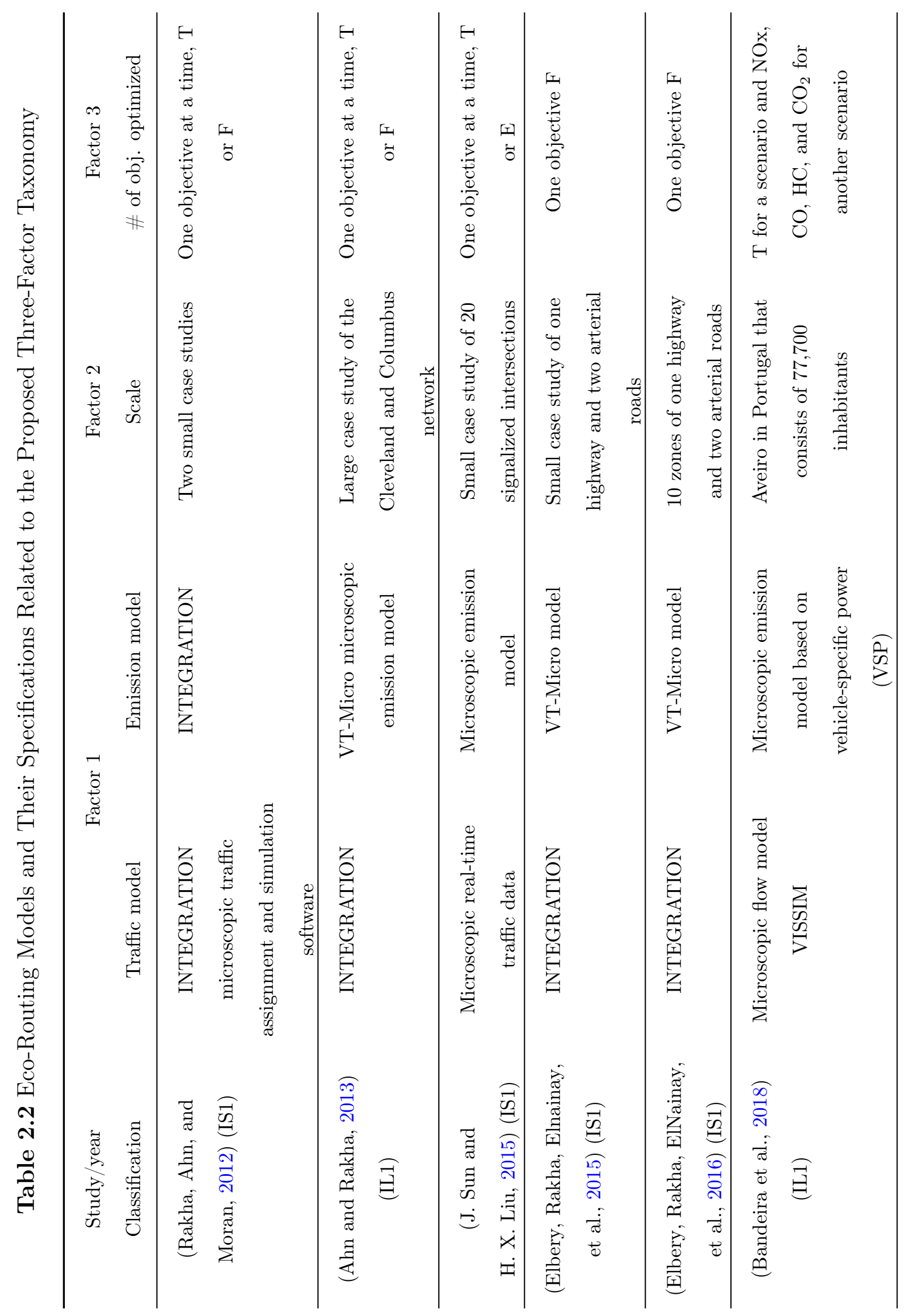




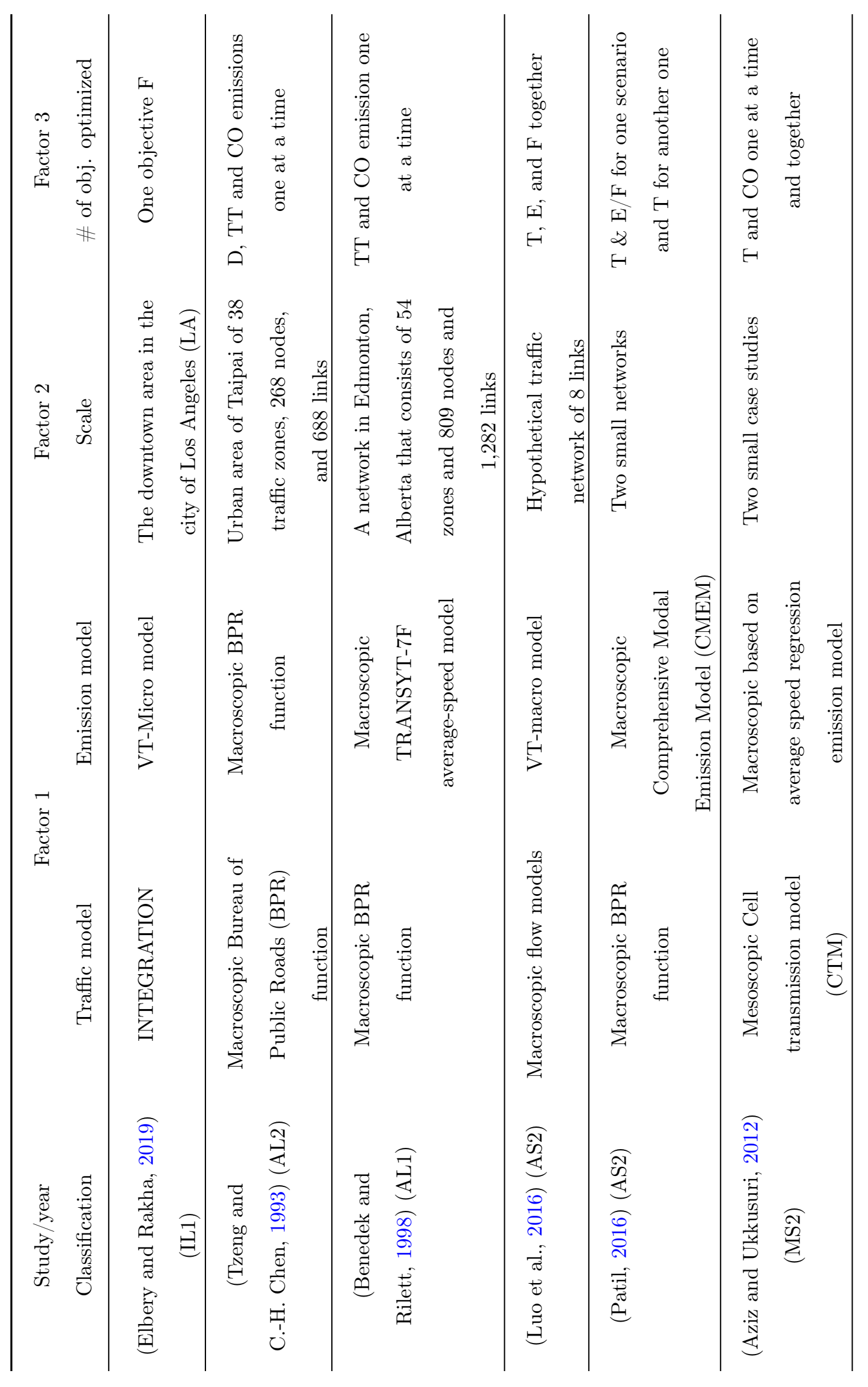




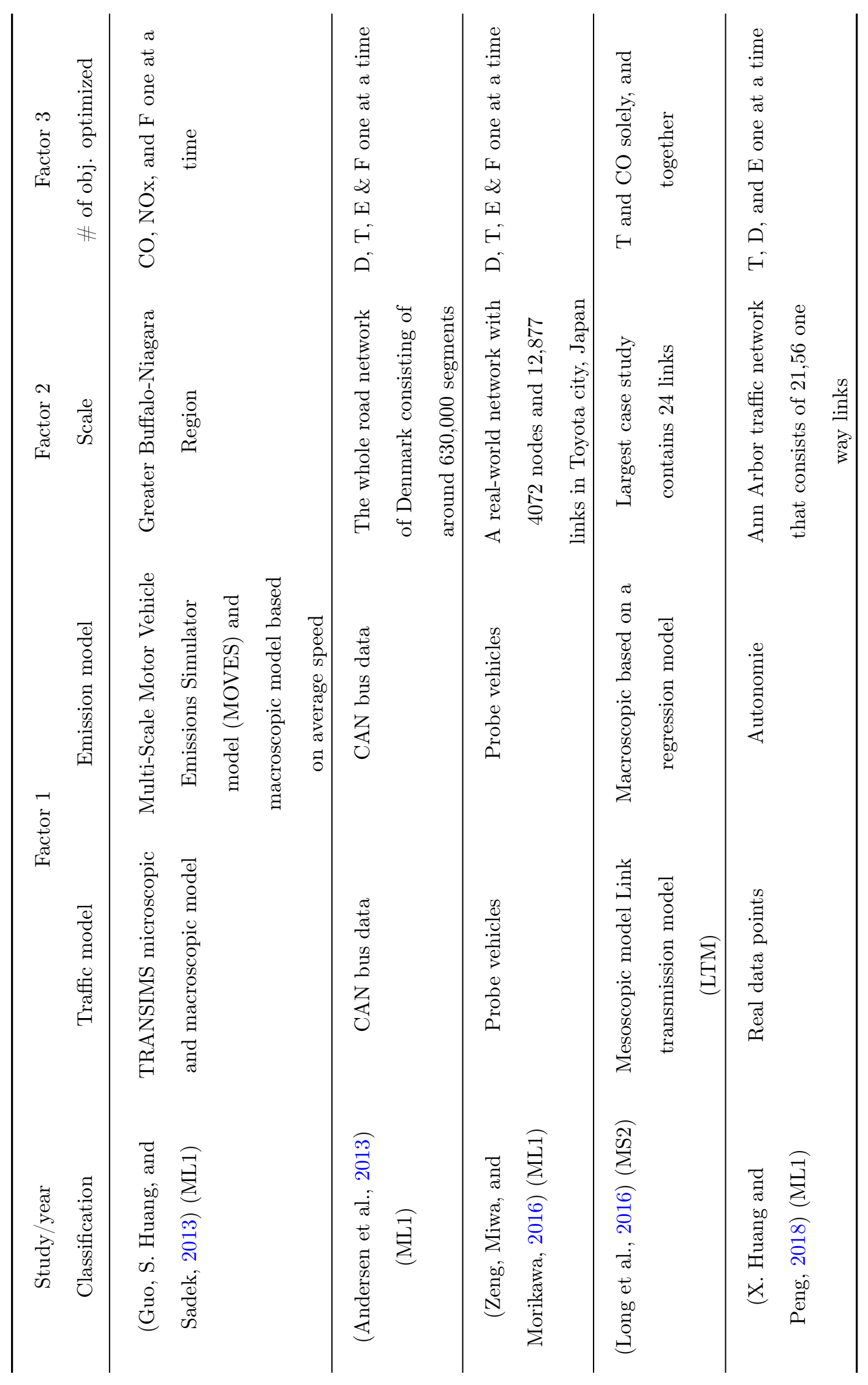


A study by Patil (2016), which belongs to the $\boldsymbol{A} \boldsymbol{S} \mathscr{2}$ classification, used average speed to estimate emissions and fuel based on regression equations. Two scenarios were set in terms of flow, free-flow speed, links length, and links capacity. The study applied UE and SO to optimize only travel time or both emissions or fuel and travel time. The major finding was that SO of a combined objective (travel time and fuel or emission) outperformed UE. Finally, it is worth mentioning that Patil (2016) tried to define the optimal speed for several pollutants.

\subsubsection{Mixed Aggregation (M) Models}

This category includes the studies that employed different levels of aggregation for traffic and emission models.

Aziz and Ukkusuri (2012) investigated the impact of incorporating CO emissions into the cost while applying dynamic routing. The study considered two different objectives for routing. One objective was based on optimizing travel time, and the other was based on optimizing travel time and emissions. A comparison between the aforementioned two scenarios was conducted. As shown in Table 2.2, the proposed model belongs to the $\mathbf{M S \mathscr { Z }}$ classification. Three scenarios for comparison purposes were applied: 1) minimizing travel time, 2) minimizing CO emissions, and 3) minimizing travel time and CO emissions. Hence, in Equation 2.1, either $T$ or $E$ were optimized for the first two scenarios, and they were optimized together for the third scenario. A normalization process, based on the monetary value, was applied when the travel time and CO emissions were optimized. The major finding of this study was that $\mathrm{CO}$ emissions were higher while trying to minimize travel time compared to the second scenario. For scenario two that aimed to minimize CO emissions, travel time increased compared to the first scenario. However, for the third scenario, a clear trade-off took place between travel time and emissions. Scenario three gave the desired results compared to the other scenarios (Aziz and Ukkusuri, 2012). 
Guo, S. Huang, and Sadek (2013) assessed the impact of green routing while using ITS that provided dynamic route guidance to travellers. The study integrated an emission model with a traffic model to define a routing strategy based on a defined objective. This study belongs to the $\boldsymbol{M L 1}$ classification. The investigation divided the study area into two parts. One section employed second-by-second trajectory data from the Transportation Analysis and Simulation System (TRANSIMS) to define emissions by using MOVES microscopic model that chose the optimal route accordingly. The other section used the average speed to estimate emissions (a macroscopic level approach). The study estimated the emission production factor (EPF) and fuel consumption factor (FCF) based on the outcomes of the MOVES model. When seeking for the green routing assignment, the EPF and FCF factors of every link would replace the cost of travel time, which was represented by Equation 2.1 for every link. Three main scenarios were applied for green routing: a) minimizing CO emissions, b) minimizing NOx emissions, and c) minimizing fuel consumption. TRANSIMS Router assigned vehicles based on the least EPF and FCF. Furthermore, for comparison purposes, another scenario was taken into account that considered the case of the shortest travel time where MOVES was used to obtain the required values for routing. The major finding was that the reduction in emissions or fuel was associated with a slight increase in travel time (Guo, S. Huang, and Sadek, 2013).

A model was proposed by Andersen et al. (2013) that gave eco-weights to a studied road network based on data collected from buses, which were called CAN buses, equipped with a GPS (Global Positioning System). Weights were given to adjust the travel time, distance travelled, and fuel consumed. Then, vehicles were routed to their destinations by applying the Dijkstra algorithm. More than 37 million CAN buses (vehicles $i$ distributed in the network) and 1.2 billion GPS records from more than 11,000 vehicles were used to define weights of the routing objectives. This study analyzed three routes: the shortest route, fastest route, and eco-route. Thus, Equation 2.1 variables ( $T, E \& F$, and $D$ ) were optimized one at a time. Since this study did not employ flow and emission models, it 
belongs to the $\boldsymbol{M L 1}$ classification. The results showed that the eco-routes varied from both the shortest and fastest routes. Furthermore, a reduction in fuel consumption was achieved with a slight increase in travel time (Andersen et al., 2013).

A study by Zeng, Miwa, and Morikawa (2016), which belongs to the $\boldsymbol{M L} \mathbf{1}$ classification, followed the logic of employing probe vehicles as in Andersen et al. (2013) to estimate emissions and used a Pareto-Optimal based algorithm for optimization. In addition to optimizing one objective at a time, $T$ was constrained and was not allowed to exceed a defined value while optimizing defined environmental objectives. The authors conducted a relative importance analysis and found that the average speed and average acceleration contributed to $85 \%$ of the relative importance of the $\mathrm{CO}_{2}$ emission model (Zeng, Miwa, and Morikawa, 2016).

A model by Long et al. (2016), which belongs to the $\boldsymbol{M S} \mathcal{2}$ classification, solved SO traffic assignment problems that minimized either total system travel time, total system CO emissions, or total travel time and CO emissions of the system. This means that $T$ and/or E in Equation 2.1 were optimized depending on the scenario. When emissions were considered in these aforementioned scenarios, the system was formulated as mixed-integer linear-programming problem (MILP). In terms of the traffic flow model, the link transmission model (LTM) was employed and was compared to the cell transmission flow model (CTM). They both proved to perform similarly. Step functions were employed to estimate link cumulative flow curves of every link and to decompose link in-flow into sub-flows. Travel time was estimated based on the sub-flows on every link. On a link, the average travel time of the inflow and sub-flow were used to provide the average speed and estimate the emissions. The model developed in this study illustrated the trade-off between minimizing the total emissions and travel time within a system (Long et al., 2016).

X. Huang and Peng (2018) investigated the eco-routing concept based on real data points. The model belongs to the $\boldsymbol{M L 1}$ classification as shown in Table 2.2. The investigated scenarios included the fastest route, shortest route, and eco-route. Thus, in Equation 2.1, $T, D$, and $E$ were optimized one at a time. The eco-routing strategy performed the best 
with respect to fuel consumption, while travel time was slightly higher than the fastest strategy. Based on this result, X. Huang and Peng (2018) added a scenario where $T$ was constrained while $E$ was optimized. A straightforward comparison was made between the optimal shortest routing, fastest routing, unconstrained eco-routing, and constrained ecorouting. With regards to the results, constrained eco-routing gave the best results compared to other strategies while considering the travel time and consumed fuel. Although there was a negligible increase in travel time, a reduction in the consumed fuel was observed compared to the shortest routing and unconstrained eco-routing strategies (X. Huang and Peng, 2018).

\subsection{Concluding Remarks}

Based on Section 2.5, it was found that when case studies were larger, most of the ecorouting studies used mesoscopic or macroscopic traffic flow models to extract the required variables for routing. These models have a major drawback, especially with the emergence of the ICT advancements. For example, when average speed was used by Long et al. (2016); Patil (2016); Guo, S. Huang, and Sadek (2013) and Aziz and Ukkusuri (2012), emissions were underestimated. Also, studies that employed regression models, as in Patil (2016); Aziz and Ukkusuri (2012) and Benedek and Rilett (1998), were dependant on vehicular speed to estimate emissions. This means that congestion in urban areas was not captured efficiently.

When it comes to the scale of case studies, small case studies were usually employed, and restrictions were made, especially with microscopic traffic and/or emission models, to reduce the complexity of the analyses (Luo et al., 2016; Long et al., 2016; J. Sun and H. X. Liu, 2015). Majority of the studies (J. Sun and H. X. Liu, 2015; Guo, S. Huang, and Sadek, 2013; Andersen et al., 2013; Benedek and Rilett, 1998) considered one routing objective, namely travel time, travel distance, consumed fuel, or emissions.

In some studies, such as Elbery and Rakha (2019); Zeng, Miwa, and Morikawa (2016) 
and Andersen et al. (2013), the use of probe vehicles is limited by securing sufficient MPR to represent traffic conditions reliably. NOx is a pollutant that has significant impacts on the public health but has been rarely considered as one of the routing objectives. Two recent studies by Elbery and Rakha (2019) and Elbery, Rakha, Elnainay, et al. (2015) incorporated one of ICT advancements, which is the vehicle to infrastructure (V2I) communication. Nevertheless, eco-routing was applied in a centralized framework, and the aim of these two studies was to examine the impact of the communication characteristics on the proposed models (Elbery and Rakha, 2019; Elbery, Rakha, Elnainay, et al., 2015). It has also been noticed that most of studies were applied in a centralized routing framework, despite its potential drawbacks. The centralized, conventional, routing frameworks has limitations related to the required large financial investment; high sensitivity to system failure; and complexity of updating the system compared to the distributed routing system (Yang and Recker, 2006). Hence, while adopting a distributed routing framework, more research related to eco-routing is urged for the management and planning of better performing transportation systems. 


\section{Chapter Three}

\section{Background Components}




\subsection{Introduction}

This dissertation aims to develop and apply anticipatory multi-objective eco-routing in a congested urban network while considering a high degree of spatial and temporal resolution. The developed eco-routing framework was compared to a myopic approach. The methodology used for the aforementioned application was agent based simulation. The first required component is a routing framework. The routing framework is where the network elements, vehicles, intersections, links, and sensors, interact based on the adopted communication protocol. In the routing framework, the traffic and emission models are the two essential components capturing the traffic and environmental characteristics at a microscopic level of aggregation. The specifications of the adopted case study are important to reflect on the scalability of this research work to another transportation network.

This chapter is organized as follows. The vehicle routing framework is presented briefly in Section 3.2. The traffic and emission models are illustrated in Section 3.3 and 3.4, respectively. The emission model requires information from the traffic model, which is discussed in Section 3.4. Finally, the specifications of the case study are presented in Section 3.5.

\subsection{Vehicle Routing Framework}

The End-to-End Connected Automated Vehicles (E2ECAV) dynamic distributed routing framework (Farooq and Djavadian, 2019) was employed in this dissertation to apply both myopic and anticipatory multi-objective eco-routing strategies and to collect data for the development of predictive models. The routing framework is where traffic and emission models interact. The data, whether related to the traffic or environmental indicators, were estimated by the traffic and emission simulators at a microscopic level. These estimations occurred at one-second time intervals. Two types of communication systems were deployed in addition to the connected automated vehicles (CAVs) in E2ECAV. The studied communication types 
include the vehicle to infrastructure (V2I) and infrastructure to infrastructure (I2I). The information was then shared with elements (link, intersection, vehicle) of the transportation system following the protocol of E2ECAV. Figure 3.1 illustrates the followed logic in the routing framework (E2ECAV) and includes the main inputs and interactions between the traffic simulator, emission simulator, and routing framework. The diagram in Figure 3.1 is divided into two main parts, which are the link and intersection. The figure also shows the processes that are applied to each component, particularly the traffic simulator, emission simulator, and vehicle routing framework. The main role of the routing framework was to receive, process, and disseminate the required information following the rules of the routing framework (E2ECAV) based on the communication, updating interval, and elements that share information. The routing framework can be observed mostly in the intersection part of Figure 3.1. At every link, when the traffic and environmental data were collected and processed to find the link cost, the aforementioned information was shared with the downstream intersection. Then, every intersection shared the information with their adjacent upstream intersections during every update interval. An updating interval was when the link cost was updated based on the data points that were measured every second. Every intersection calculated the cost of a path for every OD pair based on the given routing objective and up-to-date cost of the links. This means that all of the intersections in the network have a coherent view of the network. At each intersection, the optimal path of every OD pair is estimated and updated at an optimal time interval based on the routing objective. This time interval may vary based on the type of network and the general level of demand on the network. 


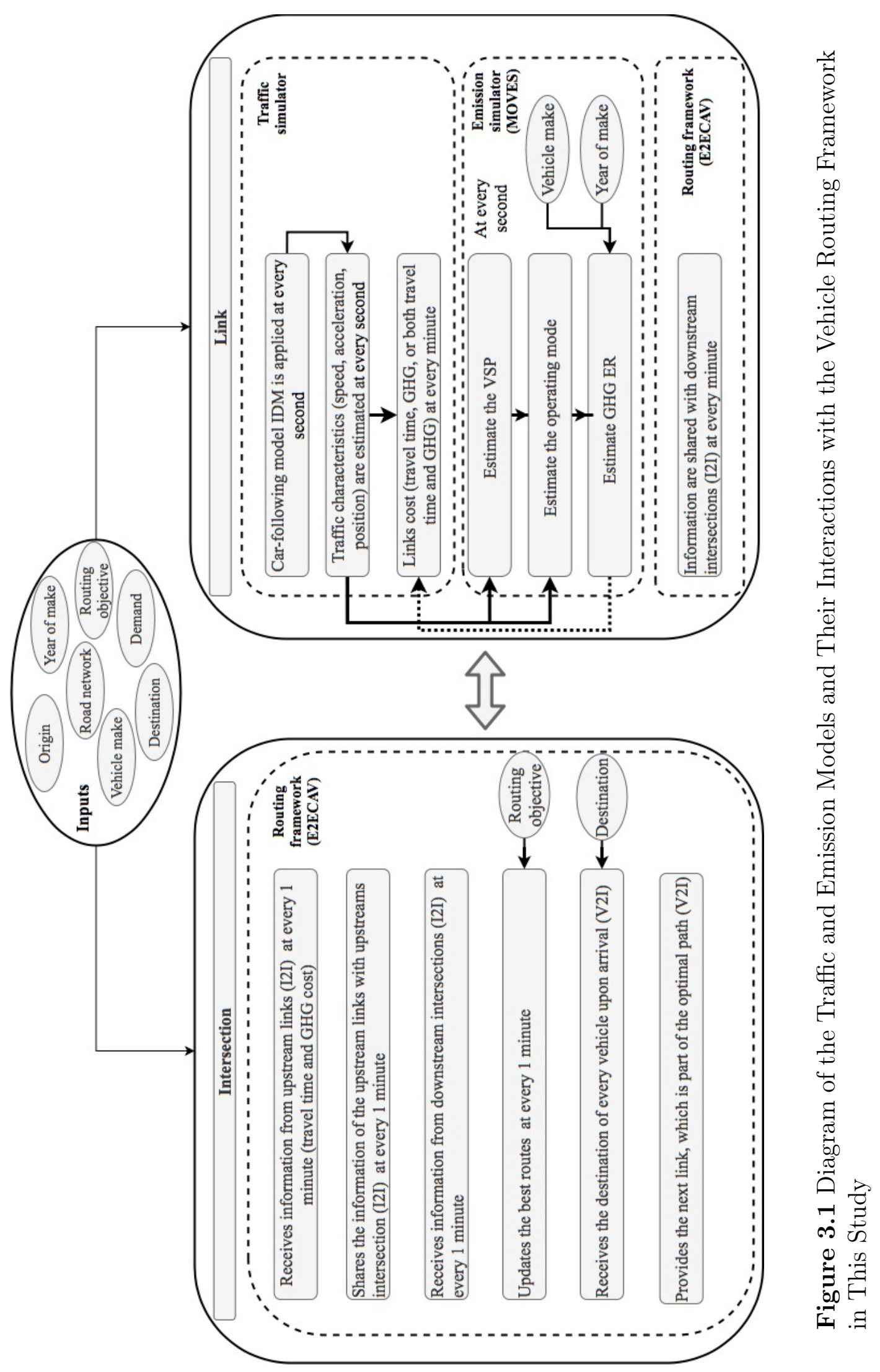




\subsection{Traffic Simulator}

In this dissertation, a microscopic agent-based traffic flow model was used (Djavadian and Farooq, 2018) to implement routing strategies, despite the associated complexity, for a congested urban network to obtain high resolution data points. Figure 3.1 demonstrates that the traffic model was only used at the link level. The second-by-second vehicular characteristics were captured and used to estimate the link cost with both the traffic and environmental indicators. The Intelligent Driver Model (IDM) (Treiber, Hennecke, and Helbing, 2000) was used to estimate the second-by-second vehicular speed from the estimated acceleration, which is expressed with Equation 3.1.

$$
\begin{gathered}
a_{i}=a_{i}^{0}\left[1-\left(\frac{v_{i}}{v_{i}^{0}}\right)^{\delta}-\left(\frac{s^{\star}\left(v_{i}, \Delta v_{i}\right)}{s_{i}}\right)^{2}\right] \\
s^{\star}\left(v_{i}, \Delta v_{i}\right)=s^{0}+T v_{i}+\frac{v_{i} \Delta v_{i}}{2 \sqrt{a^{0} b^{0}}}
\end{gathered}
$$

Where $a_{i}$ is the estimated acceleration of vehicle $i ; a^{0}$ is the maximum acceleration of 1 $\mathrm{m} / \mathrm{sec}^{2} ; b^{0}$ is the maximum deceleration of $2 \mathrm{~m} / \mathrm{sec}_{2}$ (Djavadian and Farooq, 2018); $v_{i}$ is the speed of vehicle $i ; v_{i}^{0}$ is the maximum free flow speed that can be taken by vehicle $i ; \delta$ is a calibration factor of 4 (Treiber, Hennecke, and Helbing, 2000); $s^{\star}$ is the desired gap between vehicle $i$ and its leading vehicle; $s_{i}$ is the actual gap between vehicle $i$ and its leading vehicle; $s^{0}$ is the safe spacing distance, which is 1 and $2 \mathrm{~m}$ for CAVs and human-driven vehicles (HDVs), respectively; and $T$ is the headway, which is 1 and 2 sec for CAVs and HDVs, respectively (Djavadian and Farooq, 2018). The adopted parameters, reaction time and safe spacing distance, of HDVs were the calibrated values for downtown Toronto. While for CAVs the parameters were half of the case of HDVs (Djavadian and Farooq, 2018). It is of high importance to note that the reduced reaction time and safe spacing distance of CAVs will introduce enhancements with high market penetration rates (MPRs) in addition to the impact of the routing framework. Reduced values of parameters in the case of CAVs will 
contribute to improving several traffic and environmental indicators, such as speed, density, flow, throughput, GHG, etc. On the other hand, routing makes sure to choose the optimal set of links in the network while satisfying the optimization objective. While the triggered improvements of the reduced parameters will be observed more at link level, routing impact will be at network level.

For each vehicle $i$ in the system, the IDM is a continuous function of the speed $v_{i}$, gap $s_{i}$, and speed difference $\Delta v_{i}$ to the leading vehicle. IDM has been widely and successfully used and for different types of vehicles, such as HDVs, adaptive cruise control (ACC) equipped vehicles (Kesting, Treiber, Schönhof, et al., 2008), cooperative adaptive cruise control (CACC) equipped vehicles (Zhou, X. Qu, and Jin, 2016), automated vehicles (AVs) (Djavadian and Farooq, 2018; Talebpour and Mahmassani, 2016), connected vehicles (CVs) (Talebpour and Mahmassani, 2016), and CAVs (Alfaseeh, Djavadian, and Farooq, 2018). It has been applied because it offers several benefits (Kesting, Treiber, Schönhof, et al., 2008). IDM includes the distance to the leading vehicle, and it also takes into account the difference in speed between vehicles while contributing towards a collision free feature (Kesting, Treiber, Schönhof, et al., 2008). It helps to offer a natural and smooth driving style. Acceleration oscillation is not observed in the case of new traffic conditions. It has a few representative parameters, and they are typically the desired speed and desired time gap. These parameters are defined by the driver in the case of ACC or CACC equipped vehicles. IDM can incorporate the driving behavioural aspect by changing its parameters. In particular, driving styles can be aggressive or relaxed. IDM can easily be calibrated, and it can consider cases with parameters that have different ranges or limitations, such as finite acceleration (Kesting, Treiber, Schönhof, et al., 2008). In the simulation, IDM has significantly smaller validation errors and employs a smaller number of parameters compared to other car following models (Zhu, X. Wang, Tarko, et al., 2018). Based on Zhu, X. Wang, Tarko, et al. (2018), IDM has outperformed several car-following models, such as GIPPS and Wiedemann 99 model used by VISSIM. Zhu, X. Wang, Tarko, et al. (2018) adopted urban-expressways in Shanghai to compare be- 
tween the investigated car-following models. It was found that the IDM car-following model was easier to calibrate and illustrated a better and more stable performance compared to other car-following models. Furthermore, IDM took into account the behavioural differences of different drivers (Zhu, X. Wang, Tarko, et al., 2018).

As shown in Figure 3.1, the vehicular characteristics, which are speed, acceleration, and position, were captured at every second and were used to define the cost of the links. The link cost, whether it is travel time, GHG, or both travel time and GHG, was shared with the downstream intersections based on the principles of the routing framework, E2ECAV. In the traffic simulator, the optimal updating interval $\Delta_{j}$ would vary for different networks and needs to be calibrated. This influences when the cost of the links is updated. Based on the explanation in Section 3.2, every intersection has the updated optimal path of every OD pair. When a vehicle arrives at an intersection, it declares its destination and the next link of the optimal path, based on the latest update, is provided. It is important to note that the employed traffic simulator adopted the First-In-First-Out (FIFO) logic at intersections. While focusing on the impact of connectivity, it is favourable to simplify the application and minimize the required memory and computational power. To exploit the advancements in ICT, V2I, I2I, and CAVs were utilized in the E2ECAV routing framework. The main focus in this thesis was to demonstrate the effect of communication on the traffic and environmental aspects of a transportation network. Due to the fact that links in the network are short in length and there are very few overtaking/lane changing opportunities in the congested network, the employed lateral movement model assigned the lane to a vehicle only at the beginning of the link. Considering the lateral movement only at the beginning of the links and the FIFO logic at intersections does not affect the comparative analysis between the developed different routing strategies. 


\subsection{Emission Simulator}

To maintain the same high level of resolution with the microscopic traffic flow model, MOtor Vehicle Emission Simulator (MOVES) was employed to estimate the second-by-second GHG (in $\mathrm{CO}_{2 \mathrm{eq}} \mathrm{g} / \mathrm{sec}$ ) emissions. MOVES is classified as an instantaneous model as shown in Section 2.3.2 and was developed by the United States Environmental Protection Agency (USEPA) (USEPA, 2015). According to the 2012 Canada-United States Air Quality Agreement Progress Report (International Joint Commission, 2012), Canada switched to using MOVES during the summer of 2012.

Figure 3.3 illustrates the followed steps by the emission model, MOVES, and the relationship between the emission and traffic models. The emission rate in MOVES is a fleet average value. This means that the details about the specific engine type and size were not required (United States Environmental Protection Agency, 2014). The vehicular technology with regards to the engine of CAVs would not be different from the HDVs, unless CAVs are electric vehicles (EVs). Nevertheless, the controlling aspect of CAVs would be the contributor to the enhancements in the traffic and environmental characteristics. It is worth mentioning that when CAVs are EVs in the future, emissions would be triggered by the battery charging process instead of the tailpipe. That is, emission estimation in the routing objective function would be based on emission factors $\left(\mathrm{lb} \mathrm{CO}_{2} / \mathrm{mi}\right)$. The emission factors would differ based on the electricity generation fuel source (McLaren et al., 2016). In this dissertation, light duty vehicles (LDV), including passenger cars (46\%) and passenger trucks (54\%), were only

considered. Vehicle composition data was based on the report of Ministry Transportation of Ontario in 2016 (Ministry of Transportation Ontario, 2017). Freight, public transportation, and diesel vehicles were not taken into account. The distribution of the age of the vehicles was also based on the 2016 report by the Ministry Transportation of Ontario (Ministry of Transportation Ontario, 2017). MOVES estimates the emission rate (ER) by defining the vehicle operating mode (opMode) based on the vehicle specific power (VSP) as illustrated 
in Equation 3.3. VSP represents the power demand on the engine during driving. VSP is subject to the vehicle speed, acceleration, and road grade (Koupal et al., 2005).

$$
P_{V, t}=\frac{A v_{t}+B v_{t}^{2}+C v_{t}^{3}+m v_{t} a_{t}}{m}
$$

Where $P_{V, t}$ is the VSP of speed $V$ at time $t ; v_{t}$ is the speed $(\mathrm{m} / \mathrm{sec})$ of a vehicle at time $t ; a_{t}$ is the acceleration $\left(\mathrm{m} / \mathrm{sec}^{2}\right)$ of a vehicle at time $t ; m$ is the mass of a vehicle, usually referred as "weight" (Mg); and A, B and C are track-road coefficients that correspond to the rolling resistance (in $\mathrm{kW}-\mathrm{sec} / \mathrm{m}$ ), rotational resistance (in $\mathrm{kW}-\mathrm{sec}^{2} / \mathrm{m}^{2}$ ), and aerodynamic drag (in $\mathrm{kW}-\mathrm{sec}^{3} / \mathrm{m}^{3}$ ), respectively. A, B, and C, which are based on the vehicle class, are directly affected by the pavement type (Mukherjee, 2014).

After defining the VSP of a vehicle, the operating mode was defined given the VSP, speed, and acceleration from Table 3.1. A matrix of ERs $(\mathrm{g} / \mathrm{sec})$ was derived from MOVES for all of the cases that were based on the various vehicle types, ages, and operating modes. In other words, each ER in the matrix represents the amount of emissions produced in one second by a specific vehicle of type $y$ at an age of $k$, driving in mode $j$ (opMode ID $=\mathrm{j}$ ). Figure 3.2 presents weighted emission rates for each vehicle type and calculated opMode by Equation 3.4. The vehicle ER was estimated following Equation 3.5 based on the distribution of a vehicle type:

$$
\begin{gathered}
E R_{y, j}=\sum_{k=1}^{30} E R_{y, k, j} \cdot P_{y, k} \\
E R_{j}=\sum_{y=1}^{2} E R_{y, j} \cdot P_{y}
\end{gathered}
$$

Where $y$ represents vehicle type (passenger car or passenger truck); $j$ represents opMode ID of time point $t$, which is determined from instantaneous speed, acceleration and VSP; $k$ represents the age of a vehicle, which can be at the most 30 years old (specifically, a car 
could be made between 1986 to 2016); $P_{y, k}$ is the probability of a vehicle type $y$ with an age of $k$ based on data from Ministry of Transportation Ontario (2017); $P_{y}$ is the probability of vehicle type $y$ based on the data from Ministry of Transportation Ontario (2017); $\mathrm{ER}_{y, k, j}$ is the emission rate obtained directly from MOVES of opMode ID $j$ for vehicle type $y$ with an age of $k$; $\mathrm{ER}_{y, j}$ is the emission rate of opMode ID $j$ for vehicle type $y$ that is weighted by the vehicle age; and $\mathrm{ER}_{j}$ is the emission rate of opMode ID $j$ weighted by vehicle type $y$ ( $\mathrm{Tu}$, Alfaseeh, et al., 2019).

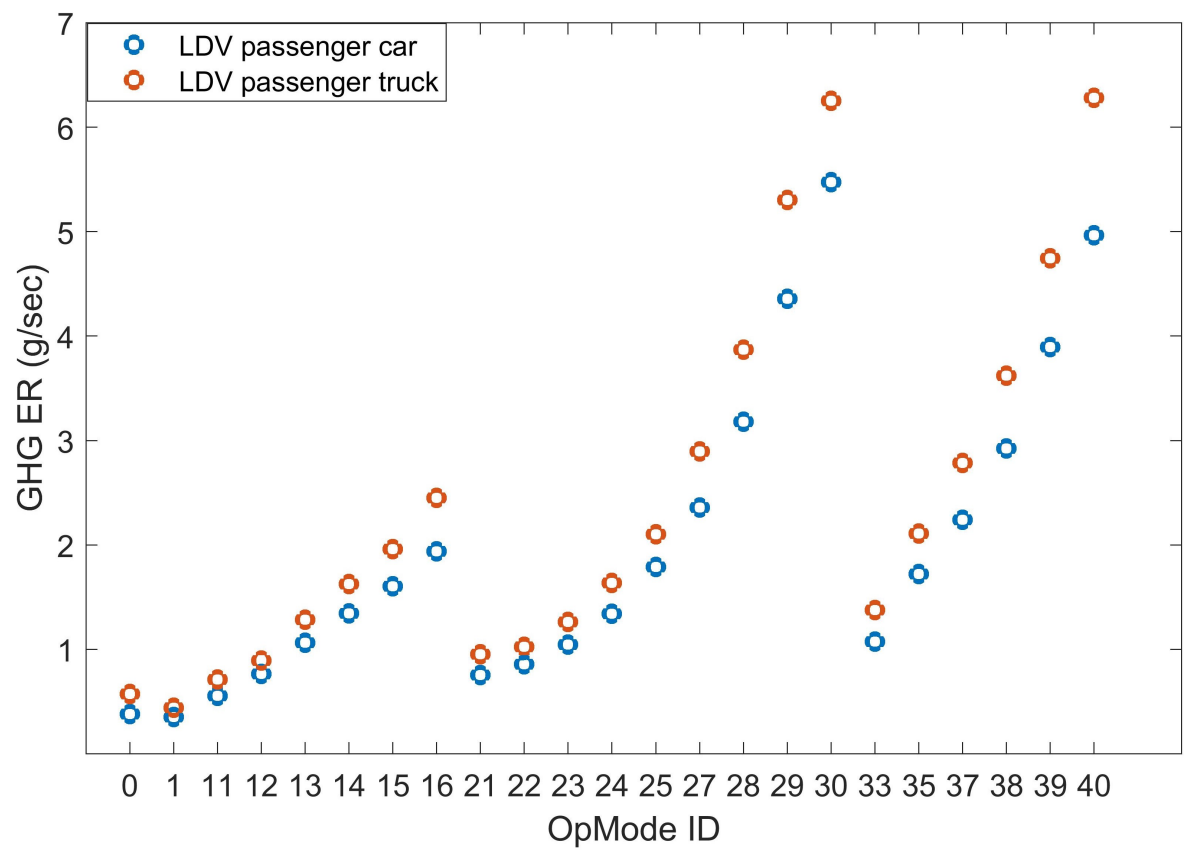

Figure 3.2 Emission Rates Versus Different OpModes for Two Different Vehicle Type

\subsection{Case Study}

For this dissertation, a congested urban network from downtown Toronto, Ontario, Canada was selected as the case study (Sweet, Harrison, and Kanaroglou, 2015; Council of Ministers Transportation and Highway Safety, 2012; Metrolinx, 2008). Figure 3.4 illustrates the area and indicates the major streets. During the morning peak period, the case study experiences 
Table 3.1 Operating Modes Based on VSP Bins

\begin{tabular}{|c|c|c|c|c|}
\hline $\begin{array}{l}\text { Operating } \\
\text { mode (opMode) }\end{array}$ & Description & $\begin{array}{c}\mathrm{VSP} \\
(\mathrm{kw} / \mathrm{Mg})\end{array}$ & $\begin{array}{l}\text { Vehicle speed } \\
\left(\mathrm{v}_{t}, \mathrm{~km} / \text { hour }\right)\end{array}$ & $\begin{array}{l}\text { Vehicle acceleration } \\
\qquad(\mathrm{km} / \text { hour-sec })\end{array}$ \\
\hline \multirow[t]{2}{*}{0} & Deceleration/braking & & & $\mathrm{a}_{t}<=-3.2 \mathrm{OR}\left(\mathrm{a}_{t}<-1.6 \mathrm{AND}\right.$ \\
\hline & & & & $\mathrm{a}_{t-1}<-1.6$ AND $\left.\mathrm{a}_{t-2}<1.6\right)$ \\
\hline 1 & idling & & {$[-1.6,1.6]$} & \\
\hline 11 & coast & $<0$ & {$[1.6,40]$} & \\
\hline 12 & cruise/acceleration & {$[0,3)$} & {$[1.6,40)$} & \\
\hline 13 & cruise/acceleration & {$[3,6)$} & {$[1.6,40)$} & \\
\hline 14 & cruise/acceleration & {$[6,9)$} & {$[1.6,40)$} & \\
\hline 15 & cruise/acceleration & {$[9,12)$} & {$[1.6,40)$} & \\
\hline 16 & cruise/acceleration & $>=12$ & {$[1.6,40)$} & \\
\hline 21 & coast & $<0$ & {$[40,80)$} & \\
\hline 22 & cruise/acceleration & {$[0,3)$} & {$[40,80)$} & \\
\hline 23 & cruise/acceleration & {$[3,6)$} & {$[40,80)$} & \\
\hline 24 & cruise/acceleration & {$[6,9)$} & {$[40,80)$} & \\
\hline 25 & cruise/acceleration & {$[9,12)$} & {$[40,80)$} & \\
\hline 27 & cruise/acceleration & {$[12,18)$} & {$[40,80)$} & \\
\hline 28 & cruise/acceleration & {$[18,24)$} & {$[40,80)$} & \\
\hline 29 & cruise/acceleration & {$[24,30)$} & {$[40,80)$} & \\
\hline 30 & cruise/acceleration & $>=30$ & {$[40,80)$} & \\
\hline 33 & cruise/acceleration & $<6$ & $>=80$ & \\
\hline 35 & cruise/acceleration & {$[6,12)$} & $>=80$ & \\
\hline 37 & cruise/acceleration & {$[12,18)$} & $>=80$ & \\
\hline 38 & cruise/acceleration & {$[18,24)$} & $>=80$ & \\
\hline 39 & cruise/acceleration & {$[24,30)$} & $>=80$ & \\
\hline 40 & cruise/acceleration & $>=30$ & $>=80$ & \\
\hline
\end{tabular}




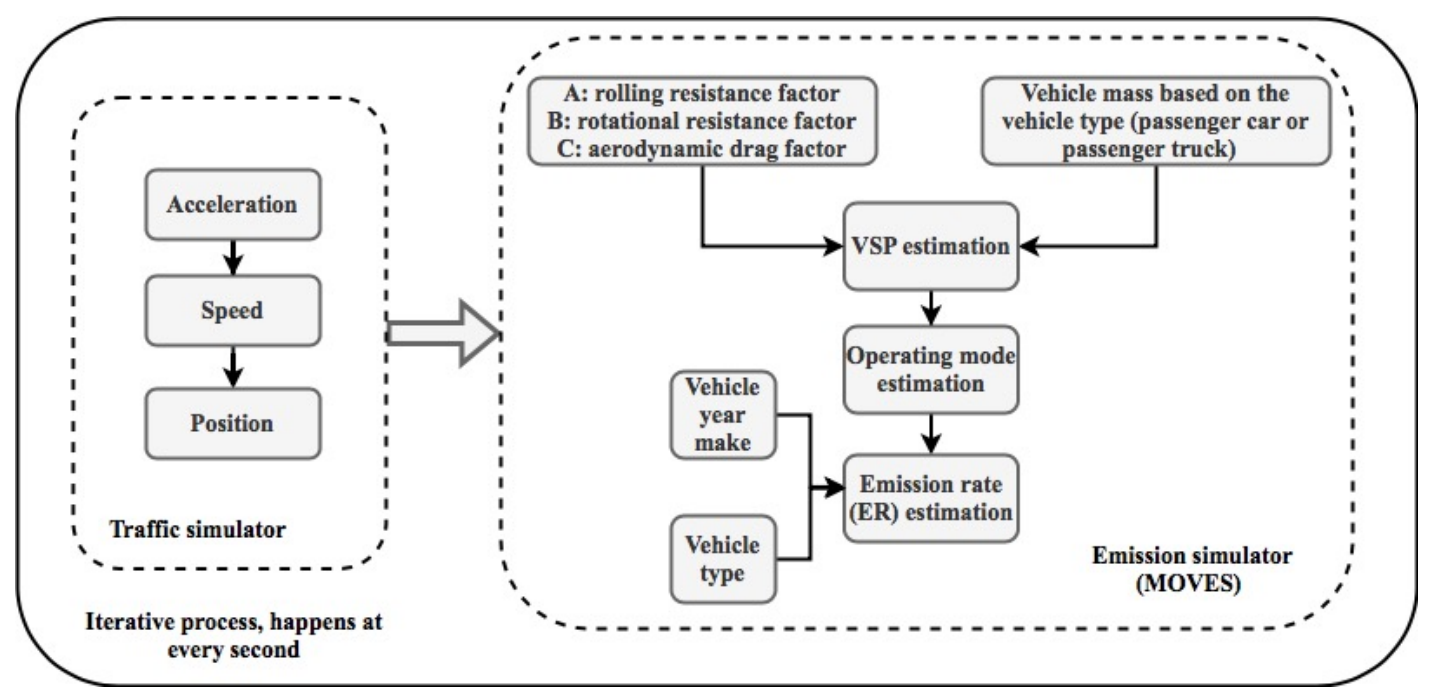

Figure 3.3 Operation of the Emission Simulator and its Relationship with the Traffic Simulator

a high level of recurrent congestion (Sweet, Harrison, and Kanaroglou, 2015). Downtown Toronto is the financial centre of Canada and has the highest job density among the major cities in the country. According to Toronto Foundation (2020), several factors contribute to the high level of congestion in Toronto. The population of Toronto has increased yearly by 1\% since 2011. Among the cities in the country, Toronto has the highest cost of living. The ownership costs are growing four times faster than income, while renting costs are growing two times faster than income over the last decade (Toronto Foundation, 2020). The network consists of 223 links and 76 nodes. Links have a high level of heterogeneity as they have different characteristics in terms of the number of lanes, free flow speed, and number of directions.

The main characteristics of the streets in the case study are presented in Table 3.2, which includes the speed limit, number of lanes per direction, number of directions, length, and number of intersections of each street. The streets are presented from west to east followed by the streets from south to north. This large case study was used throughout this dissertation as a realistic situation, and the findings can potentially be scaled and considered for other transportation networks. The vehicular demand was extrapolated for year 2014 from the 
data provided by the Transportation Tomorrow Survey (TTS) (DMG, 2011) for the period between 7:45am and 8:00am. 


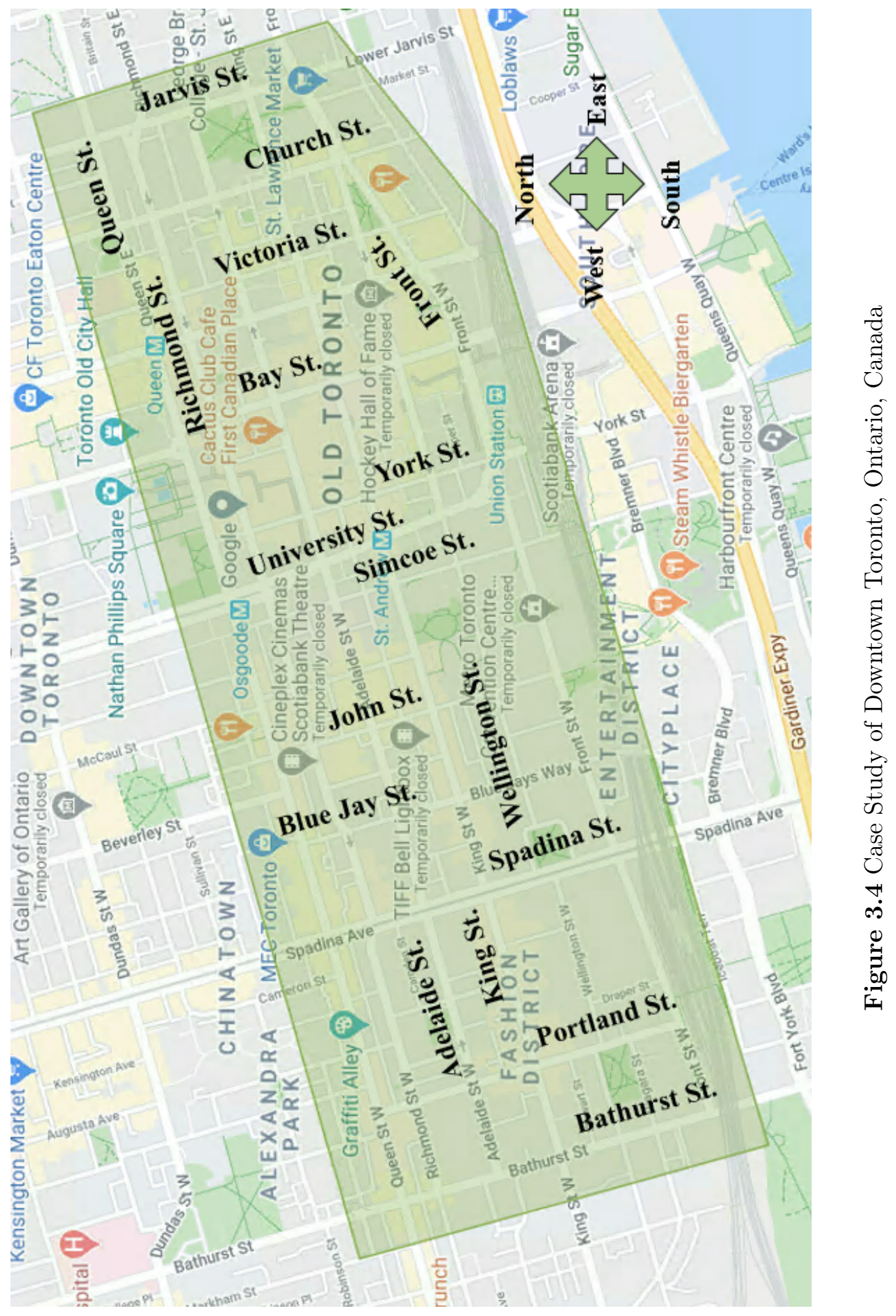


Table 3.2 Characteristics of the Links in the Case Study

\begin{tabular}{|c|c|c|c|c|c|}
\hline Street name & $\begin{array}{c}\text { Speed } \\
\text { limit }\end{array}$ & $\begin{array}{l}\text { No. of } \\
\text { lanes }\end{array}$ & Direction & $\begin{array}{l}\text { Length } \\
(\mathrm{km})\end{array}$ & $\begin{array}{c}\text { No. of } \\
\text { intersections }\end{array}$ \\
\hline Bathurst & 60 & 2 & 2 & 0.707 & 6 \\
\hline Portland & 40 & 1 & 2 & 0.707 & 6 \\
\hline Spadina & 60 & 4 & 2 & 0.707 & 6 \\
\hline Blue Jays & 40 & 2 & 2 & 0.707 & 6 \\
\hline John & 40 & 2 & 2 & 0.707 & 6 \\
\hline Simcoe & 40 & 2 & 1 & 0.707 & 6 \\
\hline University & 60 & 3 & 2 & 0.734 & 6 \\
\hline York & 40 & 4 & 1 & 0.707 & 6 \\
\hline Bay & 60 & 2 & 2 & 0.709 & 6 \\
\hline Yonge & 60 & 2 & 2 & 0.646 & 6 \\
\hline Victoria & 40 & 2 & 2 & 0.352 & 3 \\
\hline Victoria between King and Front & 10 & 1 & 1 & 0.262 & 3 \\
\hline Church & 40 & 2 & 2 & 0.552 & 6 \\
\hline Jarvis & 60 & 2 & 2 & 0.487 & 5 \\
\hline Front & 80 & 2 & 2 & 2.262 & 12 \\
\hline Front between York and Bay & 30 & 1 & 2 & 0.253 & 2 \\
\hline Wellington & 40 & 2 & 1 & 2.471 & 13 \\
\hline King & 60 & 2 & 2 & 2.471 & 13 \\
\hline Adelaide & 60 & 4 & 1 & 2.471 & 13 \\
\hline Richmond & 60 & 3 & 1 & 2.471 & 13 \\
\hline Queen & 60 & 2 & 2 & 2.471 & 13 \\
\hline
\end{tabular}




\section{Chapter Four}

\section{Analysis of End-to-End Connected Automated Vehicles (E2ECAV)}

This chapter has resulted in the following publications:

1. Alfaseeh, Lama, Shadi Djavadian, and Bilal Farooq. (2018). "Impact of Distributed Routing of Intelligent Vehicles on Urban Traffic". In 2018 IEEE International Smart Cities Conference (ISC2), IEEE.

2. Tu, Ran, Lama Alfaseeh, Shadi Djavadian, Bilal Farooq, \& Marianne Hatzopoulou (2019). "Quantifying the Impacts of Dynamic Control in Connected and Automated Vehicles on Greenhouse Gas Emissions and Urban NO2 Concentrations". In: Transportation Research Part D: Transport and Environment, 73, 142-151. 


\subsection{Introduction}

A study by Metorlinx in 2006 estimated that the cost to commuters and economy in 2031 will escalate and reach $\$ 7.8$ and $\$ 7.2$ billion, respectively, in the Greater Toronto Area (GTA) (Metrolinx, 2008). As a potential solution, intelligent transportation system (ITS) uses advancements in ICT and has the ability to control several variables, such are speed, traffic signals, and route guidance. From the environmental perspective, M. J. Barth, G. Wu, and Boriboonsomsin (2015) presented how different forms of ITS can affect the environmental aspect. ITS consists of different technologies and applications. In general, they can be classified into three essential areas: vehicle systems, traffic management systems, and travel information systems. The aforementioned three categories of ITS have the potential to mitigate the negative impact of transportation systems on the environment. This is due to the fact that the variance applications of ITS have the ability to smooth traffic flow and reduce unnecessary stop and go incidents (M. J. Barth, G. Wu, and Boriboonsomsin, 2015). ITS has been considered as the most favourable tool to improve the performance of transportation systems (Djavadian, Tu, et al., 2020; Tu, Alfaseeh, et al., 2019; Alfaseeh, Djavadian, Tu, et al., 2019; Alfaseeh, Djavadian, and Farooq, 2018; Talebpour and Mahmassani, 2016; Van Arem, Van Driel, and Visser, 2006). Particularly, it has been found that there is a proportional relationship between the efficiency of a routing framework and the performance of a transportation system (Djavadian, Tu, et al., 2020; Tu, Alfaseeh, et al., 2019; Alfaseeh, Djavadian, Tu, et al., 2019; Alfaseeh, Djavadian, and Farooq, 2018; Djavadian and Farooq, 2018; Yang and Recker, 2006). The two types of routing frameworks are centralized and distributed. Figure 4.1 shows the elements (such as the road links, intersections, vehicles, sensors, and control-

ling node/nodes) and types of communications adopted in the centralized and distributed routing frameworks. This figure demonstrates the process to gather characteristics (traffic and environmental) of road links within a network; location that these data points are processed; and potential outcome of data shared with the controlling node/nodes. 
The centralized system is the most commonly used type of framework (Luo et al., 2016; Patil, 2016; J. Sun and H. X. Liu, 2015; Rakha, Ahn, and Moran, 2012), and it has a central controller that may have a coherent view of the network, status, perturbations, and origindestination (OD) information of the vehicular demand. As shown in Figure 4.1, sensors are distributed on links to gather information on the state of a link. Examples of the sensors include passive magnetic sensors, pneumatic tube sensors, or inductive loops (GuerreroIbáñez, Zeadally, and Contreras-Castillo, 2018). In addition to sensors, vehicles equipped with vehicle to infrastructure (V2I) communication can be another source of data for a single controlling node. Data points of the whole network are sent, including the origin-destination (OD) matrices, to the transportation management centre (TMC), which is the controlling node within a system. Afterwards, the cost of the links is estimated for routing. Based on the routing objective, which may focus on the travel time or GHG, the link cost is defined. Then, the optimal path for every OD pair is specified and shared with drivers based on the communication protocol by a variable message sign (VMS) or the V2I communication. The centralized routing framework has several drawbacks (Hawas and Mahmassani, 1996). It requires a large capital investment (high price of sensors), has a high sensitivity to system failure, and can be complex to modify when the system is upgraded (Yang and Recker, 2006).

Unlike a centralized routing framework, distributed routing framework, as demonstrated in Figure 4.1, depends on many controlling nodes and several types of communication, including vehicle to vehicle (V2V), vehicle to infrastructure (V2I), and infrastructure to infrastructure (I2I). Information about road links in a network are captured by sensors and/or V2I equipped vehicles. In other words, the local information is processed at a controlling node and is shared with other elements in the network following the communication protocol of the adopted distributed routing system. Based on the cumulative efforts of the network elements to collect and share data with other elements, decisions are made locally and more reliably at controlling nodes compared to the centralized routing system, especially in the case of incidents and sudden changes. For a large scale network, distributed routing frameworks 

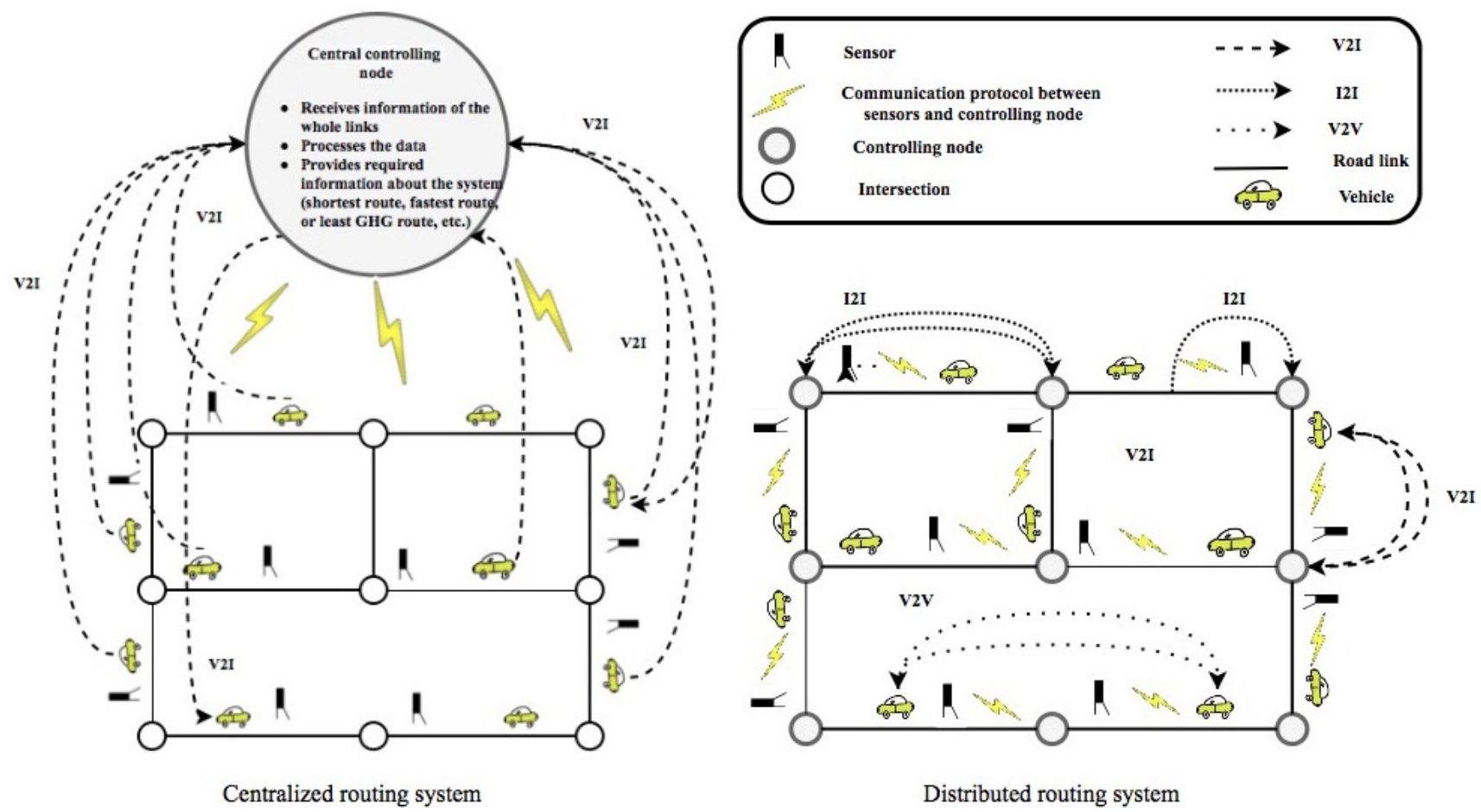

Figure 4.1 Centralized Versus Distributed Routing System

need to be adopted to efficiently route vehicles (Sha and Chow, 2019). Large networks have a high level of complexity and require the development of quick responses to perturbations, which includes accidents and congestion. For a centralized routing system, the network size is proportional to the required computational time and communication infrastructure to build a global controlling plan (Sha and Chow, 2019). A distributed routing framework overcomes this issue, and it is an attractive solution for controlling large networks (Hawas and Mahmassani, 1996). A distributed routing system offers better performance and faster responses to perturbations compared to a centralized system. A distributed routing framework is scalable unlike a centralized routing framework (Sha and Chow, 2019). The level of decentralization, whether coarser or finer, would be specified based on the available computational power, level of investment, and desired level of accuracy. In some cases, it is sufficient to apply data at the link level, while data of smaller segments within the links are required in other cases. In addition, the temporal level of resolution is another component that affects the level of 
decentralization (Hawas and Mahmassani, 1996). Several studies have examined the impact of employing distributed routing frameworks and obtained results in its favour (Djavadian, Tu, et al., 2020; Sha and Chow, 2019; Tu, Alfaseeh, et al., 2019; Alfaseeh, Djavadian, and Farooq, 2018; Claes, Holvoet, and Weyns, 2011; Yang and Recker, 2006; H. Xu, 2006; Pavlis and Papageorgiou, 1999; Hawas and Mahmassani, 1996).

In this chapter, the performance was analyzed in detail to employ the proposed Endto-End Connected Automated Vehicles (E2ECAV) dynamic distributed routing framework (Farooq and Djavadian, 2019). E2ECAV input parameters were defined including the updating interval, IDM car following model, and number of shortest paths (K-paths). The performance of E2ECAV was analyzed while considering the traffic and environmental perspectives. The traffic analysis has been conducted at two levels: link and network. The impact was assessed for different MPRs of CAVs with various traffic conditions, such as highly congested, congested, and uncongested. The throughput, speed, density, flow, total greenhouse gas (GHG) emissions, and nitrogen oxide (NOx) emissions were the performance indicators for the different MPRs of CAVs and traffic conditions. Figure 4.2 presents an overview of this chapter and its relationship with the other chapters in this dissertation. E2ECAV was adopted as the routing framework to apply the myopic routing, which is discussed in Chapter 5. This framework collected data points for the developed predictive models, and the details of this process are provided in Chapter 6. Anticipatory routing was added to E2ECAV and is described in detail in Chapter 7.

This chapter is organized as follows. Section 4.2 briefly provides background information about sensing technology, intelligent vehicles, and the distributed routing framework. The adopted traffic and emission models to examine E2ECAV are illustrated in Section 4.3. Section 4.4 demonstrates the specifications of the case study. The description of the assessed E2ECAV distributed routing system is in Section 4.5. The optimal input parameters, updating interval, IDM parameters, and number of the optimal paths (K-paths) applied within E2ECAV are discussed in Section 4.6. E2ECAV is analyzed in Section 4.7. The perfor- 
mance of routing the traffic is studied in Section 4.7.1, while the environmental perspective is investigated in Section 4.7.2. Finally, concluding remarks are presented in Section 4.8.

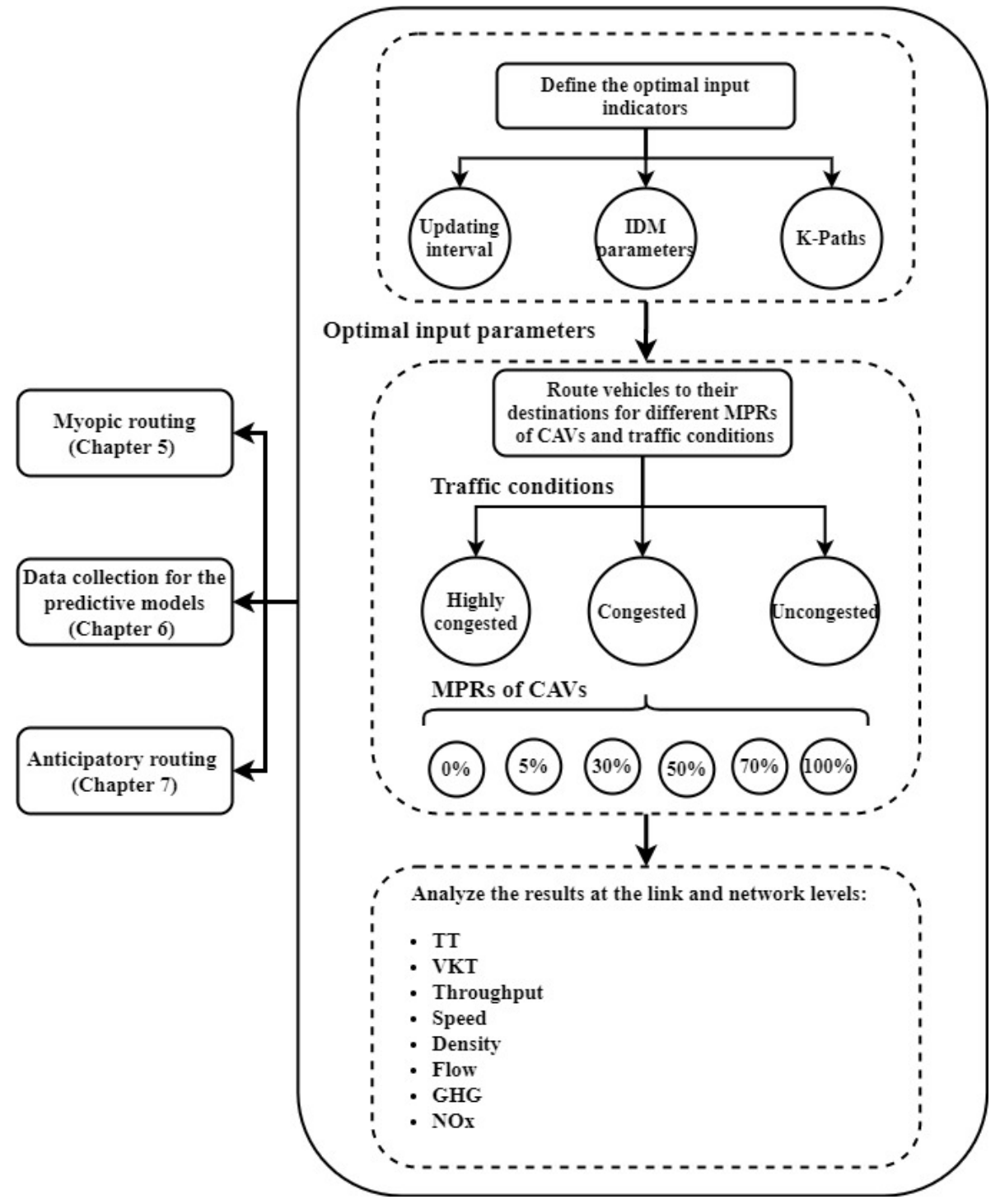

Figure 4.2 Chapter Four Framework

\subsection{Related Literature}

During the last few decades, advancements in ICT have been applied to transportation systems and have modified cities in different ways. Two forms of technologies were generally used 
to improve transportation systems. The first technology uses wireless transmissions to send data from sensors to external data processors. The second technology is vehicle-based and can be seen by the development of intelligent vehicles (Talebpour and Mahmassani, 2016). Sensing platforms are classified into two categories. The intra-vehicular sensing platform is the first category, and it collects information about the conditions of a vehicle. The second category is the urban sensing platform, which is used to collect information about traffic conditions. The sensing technology is an important element for data collection during the V2V and V2I communications that are used in distributed routing frameworks (GuerreroIbáñez, Zeadally, and Contreras-Castillo, 2018). V2V and V2I communications allow the traffic state to be estimated more reliably and contribute to more robust decisions during a trip, and they offer a more efficient transport system as a result (Talebpour and Mahmassani, 2016). The gathered information is used by the controlling node or nodes to be used for any controlling or managing process, such as estimating optimal routes (Guerrero-Ibáñez, Zeadally, and Contreras-Castillo, 2018).

Control technology with intelligent vehicles had grabbed the attention of researchers during the last few decades. Adaptive cruise control (ACC), as introduced by Mitsubishi in 1993, is one of the first forms of driving assistance systems where vehicles change their speed to maintain a specific and safe spacing distance (Pananurak, Thanok, and Parnichkun, 2009). ACC is used to enhance the driving comfort by automatically reacting to a preceding vehicle. Afterwards, the cooperative adaptive cruise control (CACC) was introduced, and it is an extension to ACC (Xiao, M. Wang, and Arem, 2017). CACC equipped vehicles have the ability to communicate with other vehicles via the V2V communication to maintain a safe and constant spacing distance. Since the CACC equipped vehicles can coordinate and communicate with other vehicles, they contribute to better performing transportation system compared to ACC equipped vehicles (Xiao, M. Wang, and Arem, 2017). Automated vehicles $(\mathrm{AVs})$ are another form of intelligent vehicles that can be defined as driverless vehicles according to the United States Department of Transportation (USDOT) (NHTSA, 2013). 
Automation has five levels (Society of Automotive Engineers, 2018) as illustrated in Figure 4.3. Level 0 is when automation is not available, and the driver is responsible for the

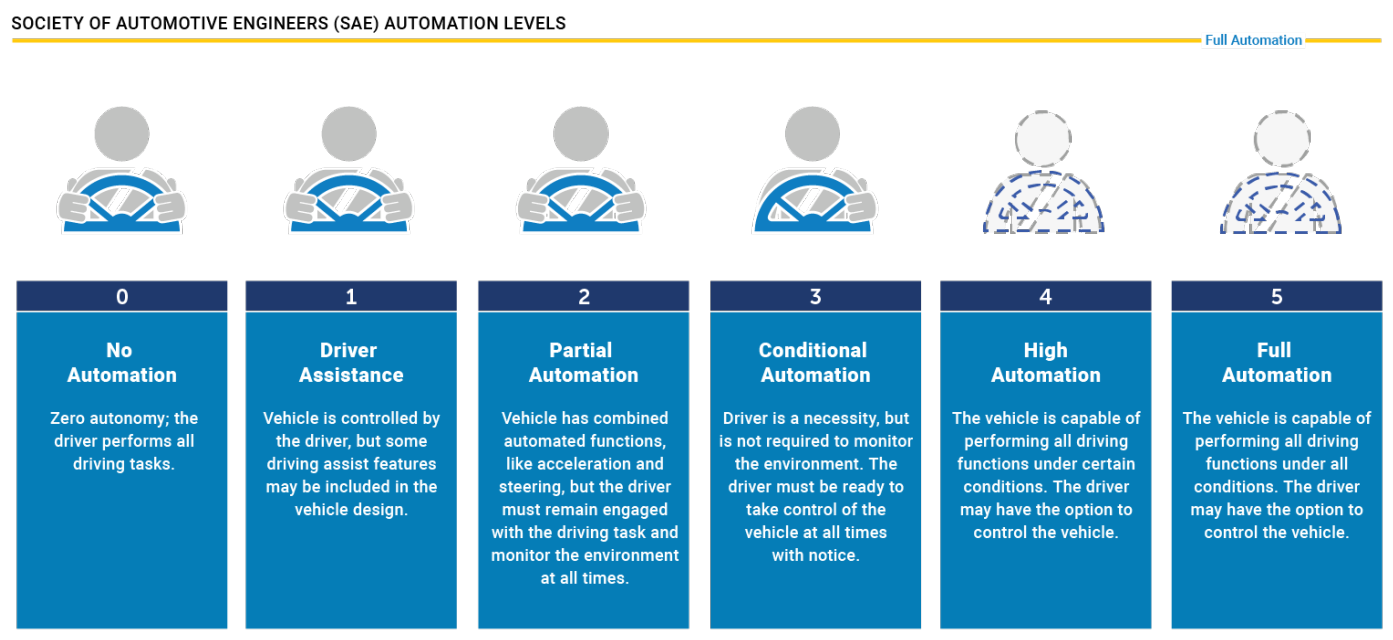

Figure 4.3 Automation Levels of Vehicles (Society of Automotive Engineers, 2018)

main vehicle controls, including braking, steering, throttling, and motor power, at all times. The driver is completely responsible to monitor the road. Level 1 is when an advanced driver assistance system (ADAS) on the vehicle can sometimes assist the human driver with either steering or braking/accelerating, but assistance to both actions cannot be used at once. Examples of level 1 automation are ACC and CACC. Level 2 is when an advanced driver assistance system (ADAS) on the vehicle can itself actually control both steering and braking/accelerating at the same time under some circumstances. The human driver must continue to pay full attention at all times and perform the rest of the driving task. Examples include, but are not limited to, Tesla Autopilot and Volvo Pilot Assist. Level 3 is also called conditional automation and is when an automated driving system (ADS) on the vehicle can itself perform all aspects of driving under some circumstances. In those circumstances, the human driver must be ready to take back control at any time when the ADS requests the human driver to do so. In all other circumstances, the human driver performs the driving task. Level 3 vehicles can stop at stop signs; obey traffic signals and signs; and deal with 
complex traffic situations without any driver intervention. However, drivers must pay attention in order to take over at any time. Level 4 is when an automated driving system (ADS) on the vehicle can do all driving tasks and monitor the driving environment under certain circumstances. The human driver does not need to pay attention if he or she does not want to intervene. Level 5 is when an automated driving system (ADS) on the vehicle can do all the driving in all circumstances. This means that there is not a driver and only passengers are in the level 5 automated vehicle (Society of Automotive Engineers, 2018). Connected vehicles $(\mathrm{CVs})$ are another type of intelligent vehicles that are capable of communicating with other vehicles via V2V communication and/or V2I communication (Harding et al., 2014). V2V communications use on-board dedicated communication devices, such as a short-range radio, to disseminate messages to other vehicles about the characteristics of a vehicle, such as speed, heading, brake status, and other information. The communication devices also receive the same information with a range and "line-of-sight" capabilities that exceed by nearly twice the range of current and near-term systems. V2V communications outperform the sensors, radars, or cameras in terms of detecting incidents in the network because they have a long transmission range and the ability to see through vehicles and around corners. On the other hand, V2I communication goes beyond the safety aspect to incorporate the traffic and environmental performance factors (Harding et al., 2014). Lastly, the most advanced type of intelligent vehicles is connected automated vehicle (CAV), which has a certain automation level as demonstrated in Figure 4.3 with the communication ability (Djavadian and Farooq, 2018; Alfaseeh, Djavadian, and Farooq, 2018). During a trip, CAVs are not the decision makers. In the case of CAVs when level 5 of automation is adopted, the $100 \%$ compliance to management and controlling decisions in the network, paves the way towards achieving the System Optimal (SO) traffic assignment (Alfaseeh, Djavadian, and Farooq, 2018; Djavadian and Farooq, 2018).

Several studies have investigated the impact of employing ACC and CACC (Milanés and Shladover, 2014; Jerath and Brennan, 2012; Van Arem, Van Driel, and Visser, 2006; Vander 
Werf et al., 2002) on the traffic characteristics of highways. Mainly, these studies showed that the CACC outperformed the ACC by improving the traffic characteristics, throughput, capacity, and speed. It was also noticed that for the same MPR, ACC was associated with dramatically more speed oscillation compared to when CACC was adopted. Since the CACC equipped vehicles were able to communicate their information with other vehicles, the following vehicles were aware of the traffic state, unlike the case of ACC equipped vehicles. It is worth mentioning that speed oscillation adversely affects both the string stability (disturbance in traffic variables should not spread along the platoon) and environmental aspect (Alfaseeh, Djavadian, Tu, et al., 2019) due to the quasi-convex relationship between both speed and GHG or nitrogen oxide (NOx) emissions (Djavadian, Tu, et al., 2020). In particular, NOx is a pollutant that negatively contributes to the public health (Price et al., 1997). This asserts that connectivity is a major feature that has positive impacts on the traffic (Milanés and Shladover, 2014; Jerath and Brennan, 2012; Van Arem, Van Driel, and Visser, 2006; Vander Werf et al., 2002) and environmental indicators (Alfaseeh, Djavadian, Tu, et al., 2019). Olia et al. (2018) assessed the impact of different combinations of CAVs, AVs, and human-driven vehicles (HDVs) on a highway. The authors found that using 100\% AVs and $100 \%$ CAVs improved the vehicle capacity by $9 \%$ and $300 \%$, respectively, compared to when $100 \%$ HDVs were employed (Olia et al., 2018). The significant positive impact of CAVs compared to the AVs is due to the triggered string stability by the connectivity feature. The highway case study can further contribute to the performance of CAVs since it offers a long distance of uninterrupted flow and allows for high speeds with less speed oscillations. Talebpour and Mahmassani (2016) investigated the impact of employing AVs and CVs on the traffic flow stability and string stability on a highway and considered different MPRs for the AVs and CVs in their study. The CVs used two types of communication: V2V and V2I. It was found that AVs contributed to a better flow stability compared to the same MPR of CVs, while CVs contributed to a better string stability compared to the same MPR of AVs. Talebpour and Mahmassani (2016) also found that the traffic throughput improved for 
certain MPRs. It is important to note that certain MPRs of any type of intelligent vehicle may contribute to more re-routing and worse results and vise versa (Alfaseeh, Djavadian, Tu, et al., 2019).

To route vehicles in a centralized routing system, the link cost should be estimated based on the global view of the network (Sha and Chow, 2019). However, the required computational power and communication infrastructure to build a global controlling plan are subject to the size of a network. This can be very challenging for large and/or complex networks. Thus, distributed routing frameworks have been suggested as a solution due to their ability to deal with local data; communicate with other elements in the network; and build a view of the network that is sufficient to make controlling decisions. Distributed routing systems, which employ different types of communication, estimate the link cost with the local data. Elements in a distributed routing system share information with each other to develop a coherent view of the network for a reliable routing. Hawas and Mahmassani (1996) compared centralized to distributed routing frameworks with different traffic incidents, such as a lane blockage, at different levels and durations. It was found that the distributed routing framework outperformed the centralized framework by managing a greater capacity of vehicles. The distributed routing framework was able to update the routes based on local information compared to the centralized routing framework, which did not change from the original route. For the distributed routing system, traffic perturbations were captured quicker since they were reflected on the local link cost. For the centralized routing system, worse outcomes could have been due to delays and the level of accuracy with the data (Hawas and Mahmassani, 1996). Yang and Recker (2006) examined the impact of intervehicle communication (IVC). They considered different MPRs of IVC equipped vehicles and various traffic conditions. They adopted two case studies: arterial streets and a highway corridor. It was noticed that the average travel time was less when MPRs of equipped vehicles were higher than a threshold of 1-2\% of equipped vehicles (Yang and Recker, 2006). Lee and Park (2008) examined the effect of routing based on vehicle-infrastructure integration (VII). 
The VII communication feature provides communication between vehicles and the roadside infrastructure to increase the safety, traffic efficiency, and convenience of the transportation system. Four routing strategies were considered that required different information from the VII feature: 1) the latest travel time-based guidance (LTG) required the latest link travel time; 2) the averaged travel time-based guidance (ATG) required the average of link travel time; 3) the routing travel time-based guidance (RTG) required the travel times of individual vehicles on links and their directional movements at intersections; and 4) the predicted travel time-based guidance (PTG) required the origin-destination table. Lee and Park (2008) compared these guidance strategies to the base case, which was no guidance, and found all of the route guidance strategies offered enhancements based on the vehicle hours travelled, vehicle speed, and throughput. However, the vehicle kilometers travelled increased for all of these route guidance strategies. The best route guidance strategy was ATG as it contributed to a decrease in the vehicle hours travelled, speed, and throughput respectively by $26 \%, 55 \%$, and $20 \%$ compared to the base case of no route guidance. The vehicle kilometres travelled increased by $16 \%$ compared to the base case (Lee and Park, 2008). Sha and Chow (2019) also

compared centralized and distributed routing frameworks for managing urban networks with users that were aware of prevailing traffic conditions. The authors examined the impact of different MPRs of users, who had the choice to re-route according to the network state. Small case studies and a microscopic traffic model were selected. They found that the performance of the proposed distributed routing systems was proportional to the compliance MPR of drivers to re-routing based on the traffic condition.

\subsection{Traffic and Emission Models}

An agent-based microscopic traffic simulation was employed that captured the second-bysecond vehicular characteristics (Djavadian and Farooq, 2018). The link cost was updated at every one minute interval, which was found by Alfaseeh, Djavadian, and Farooq (2018) to be 
the optimal. MOVES (United States Environmental Protection Agency, 2014) was used as the emission model to assure compatible and high resolution data points for GHG emissions. Further details related to the traffic and emission models can be found in Sections 3.3 and 3.4 , respectively.

\subsection{Case Study}

Unlike most of the previous work from the literature on distributed routing systems, this dissertation studied a highly congested urban network that was based on a section of downtown Toronto. The simulation was based on the demand of the morning peak hour from 7:45am to 8:00am and stopped when all the vehicles reached their destinations. As demonstrated in Section 3.5, the characteristics of the links indicate a high level of heterogeneity due to the number of lanes, speed limits, and street directions within the network.

\subsection{Description of E2ECAV}

E2ECAV employed intelligent intersections and level 5 CAVs. Two types of communication were used: V2I and I2I. With E2ECAV, the link related indicators, such as speed, density, and flow, were updated every one minute (Farooq and Djavadian, 2019), which was found to be an optimal interval by Alfaseeh, Djavadian, and Farooq (2018). The CAVs shared their information with the links they traversed via the V2I. The link cost was estimated, whether it was the travel time, GHG, or both travel time and GHG. The information was then transferred to the downstream intersections via the I2I communications. For a coherent view of the network, the intersections in the network shared their information with the other adjacent upstream intersections via the I2I communication. The cost was estimated for every origin-destination (OD) pair at every updating interval by each intersection. CAVs were guided in the network as they did not have information about the traffic conditions 
on the links (Farooq and Djavadian, 2019). When a vehicle arrived at an intersection, it declared its destinations, and the next link on the optimal route was provided to the vehicle based on the up to date cost of the paths. Further details related to E2ECAV can be found in Section 3.2. The interactions between the traffic emission simulators in E2ECAV routing framework are presented in Figure 3.1.

\subsection{E2ECAV Parameters Analysis}

In this section, the optimal input parameters of E2ECAV were defined for the updating interval, IDM parameters, and optimal K-Paths. Table 4.1 presents the input parameters and their values. First, the updating interval was examined for the same level of demand while keeping the other input parameters constant. Then, the regular and reduced IDM sets were examined. Finally, the optimal number of K-Paths was examined while using the optimal updating interval and IDM set parameters. To account for stochasticity, five trials with different random seeds were run for every scenario as recommended by Law and McComas (1991).

\subsubsection{Updating Interval}

The updating interval is an element that has a significant impact on the routing efficiency. The updating interval defines the frequency to update the link cost. Changes in the traffic conditions should be captured in a timely manner to reliably estimate the link cost. While considering the required computational power and memory, three updating intervals were assessed: 30,60, and 180 seconds. Updating intervals less than 30 seconds were not included in this analysis since shorter intervals require more computational power and delays can occur while data is sent to the controller. For the free flow speed, the travel time on links in the case study ranges from 0.07 to 0.8 minute. However, when the network is congested, it takes a longer time. The 30, 60, and 180 second updating intervals were examined to 
Table 4.1 The Investigated Input Parameters

\begin{tabular}{|l|lll|}
\hline Input parameter & \multicolumn{3}{|c|}{ Values } \\
\hline Updating interval (second) & 30 & 60 & 180 \\
\hline Safe spacing distance (meter) & 1 & 2 \\
\hline Reaction time (second) & 1 & 2 \\
\hline K-Paths & 1 & 2 \\
\hline
\end{tabular}

comprehend the magnitude of different updating intervals.

Figure 4.4a shows the mean travel time (TT) and vehicle kilometres travelled (VKT), and Figure $4.4 \mathrm{~b}$ shows the throughput of the different updating intervals. The mean TT, mean VKT, and throughput of a 30-second updating interval were similar to the 60-second updating interval. The average TT for an updating interval of 60 seconds surpasses the 30 -second updating interval by only $2 \%$. A one minute interval was able to efficiently detect the changes within the network while requiring less memory and computational power. An updating interval of 30 seconds takes double the time to simulate a scenario compared to a 60-second updating interval. As illustrated in Figure 4.4, the 180-second updating interval introduces a significant deterioration in the three performance indicators: TT, VKT, and throughput. This is stemmed from the fact that a three-minute period is too long to capture the changes in the traffic condition. Even when the network is congested and a longer time is required to travel the links, a 3 minute interval is unable to detect the changes in the network. This is reflected on the throughput analysis, in which the vehicles required 10 more minutes to reach their destinations compared to the one-minute updating interval. When 3 minute updating interval was adopted, changes in congestion level were not captured in time, which means the link cost was either underestimated or overestimated contributing to the aforementioned deterioration. When the link cost was underestimated, a lot of vehicles were sent to congested links causing more congestion. When the link cost was overestimated, 
the links were averted and were not chosen to be part of the optimal paths.

Based on these results, one-minute updating interval was used for the myopic routing, data collection for the predictive models, and anticipatory routing in Chapters 5, 6, and 7, respectively. One minute interval gives results that are very much comparable to the 30 second updating interval while requiring less computational power and memory.

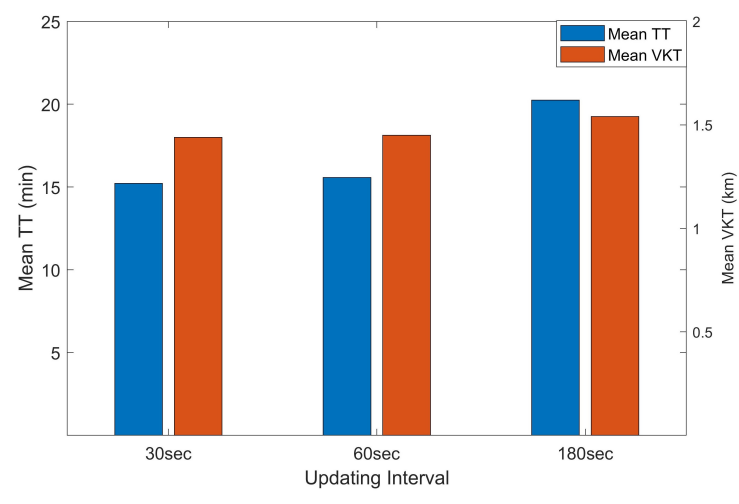

(a)

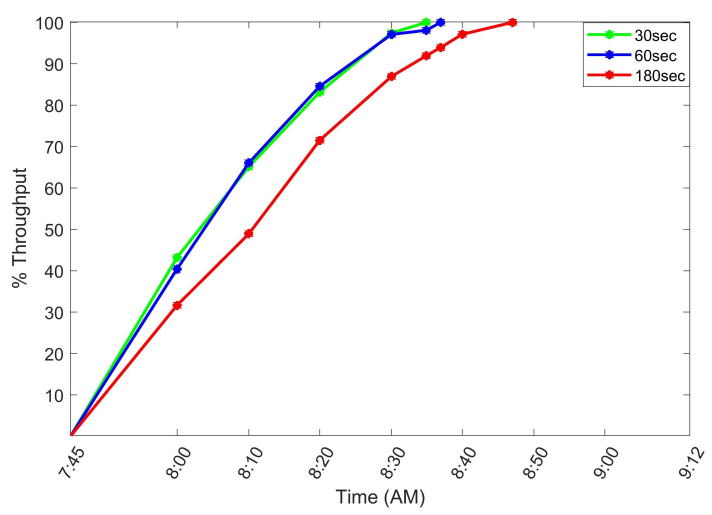

(b)

Figure 4.4 The Impact of Different Updating Intervals on the a) Mean TT, Mean VKT and b) Throughput

\subsubsection{IDM Parameters}

A microscopic agent-based traffic simulator was used in this dissertation (Djavadian and Farooq, 2018). The second-by-second vehicular characteristics, namely speed, acceleration, and position, were estimated and saved at every second. IDM (Treiber, Hennecke, and Helbing, 2000) was adopted as the car-following model in the traffic simulator. The related details can be found in Section 3.3. IDM calculates the longitudinal speed of vehicles based on the change in acceleration at every second. The estimated acceleration depends on the safe spacing distance and reaction time as provided in Equation 3.1. Hence, two different sets of the IDM parameters were examined as illustrated in Table 4.1. HDVs follow a different logic compared to AVs. HDVs require time to react, but AVs can react instantaneously 
(Talebpour and Mahmassani, 2016). The reaction time of an HDV varies from 0.7 to 1.5 seconds (Green, 2000). Talebpour and Mahmassani (2016) considered the reaction time of an AVs to be half of a HDV. It was assumed in this part of the analysis that the safe spacing distance and reaction time of HDVs were 2 meters and 2 seconds, respectively. This is the regular set of IDM parameters in Table 4.1. However, for CAVs, two sets were examined: regular and reduced. The regular set was the same as HDVs, while the reduced IDM set was half value of the regular set. Figures $4.5 \mathrm{a}$ and $4.5 \mathrm{~b}$ show that the mean TT, mean VKT, and throughput of the reduced IDM set are comparable to the regular IDM set. Yet, the reduced IDM set contributes to a reduction in the mean TT and VKT by roughly $4 \%$ and $1 \%$, respectively, compared to the regular IDM set. The throughput indicates that it takes about 2 minutes of less time to load and unload the network with the reduced IDM parameters. The negligible enhancement in the case of reduced IDM set is linked to the adopted case study, which is an urban network. The short length and stop-and-go nature of the links must have limited the improvements. The reduced IDM set was employed throughout this dissertation to exploit the characteristics of automated vehicles, and a better performing transportation system can be found with vehicles that have lower safe spacing distances and reaction times.

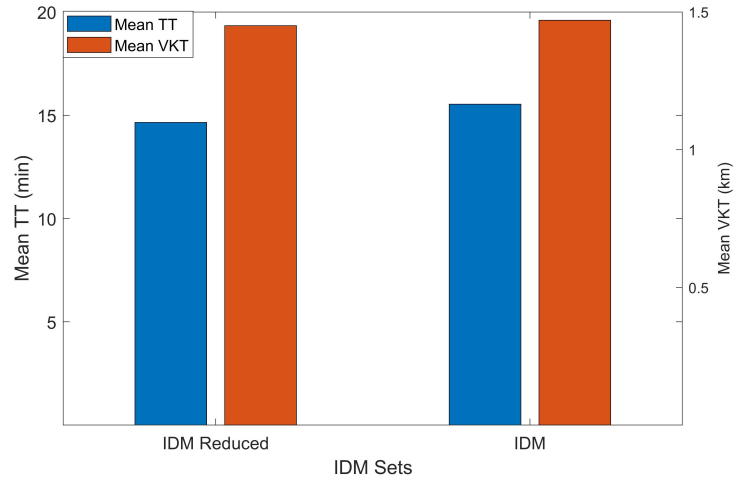

(a)

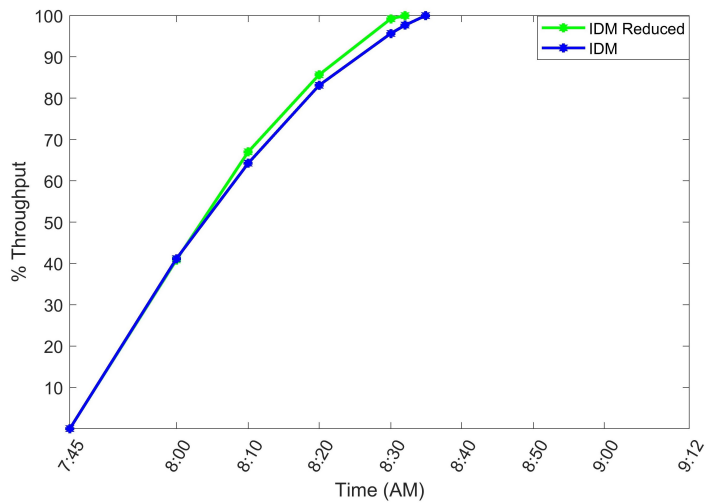

(b)

Figure 4.5 The Impact of Different IDM Sets on the a) Mean TT, Mean VKT, and b) Throughput 


\subsubsection{Shortest Paths (K-Paths)}

Another essential input is the number of optimal paths (K-Paths) for vehicles as they are routed to their destination. The impact of using one and two K-Paths was analyzed. When a vehicle arrives at an intersection, it declares its destination. The intersection has the option to provide the vehicle with the next link of the first optimal route or to choose one randomly from two optimal routes. More computational power and memory is required to calculate and save information on additional optimal routes.

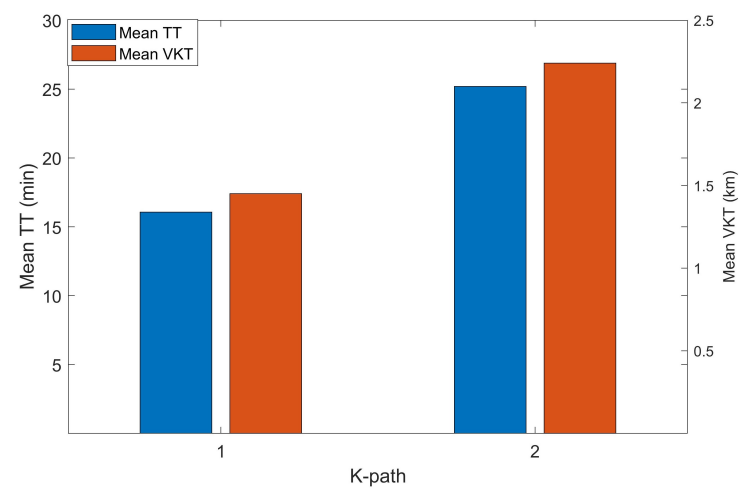

(a)

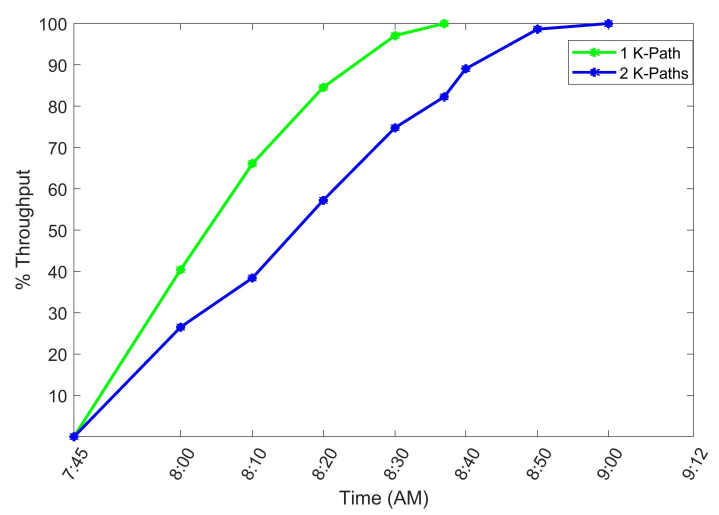

(b)

Figure 4.6 The Impact of the Number of Optimal K-Paths on the a) Mean TT, Mean VKT, and b) Throughput

Figures $4.6 \mathrm{a}$ and $4.6 \mathrm{~b}$ show that there is a significant deterioration in the mean TT, mean VKT, and throughput when there are two K-Paths. The mean TT and VKT increased by $66 \%$ and $56 \%$, respectively, for two K-Paths. When more than one optimal route is provided, more re-routing was triggered that resulted in longer travel distances, and the additional time spent in the network is shown by Figure 4.6b. One K-Path was used throughout this dissertation. 


\subsection{Results and Discussion Using Optimal Parameters}

In this section, the performance of E2ECAV, while relying on the optimal parameters, is discussed based on the traffic and environmental perspectives. MPRs of 0\%, 5\%, 30\%, $50 \%, 70 \%$, and 100\% CAVs were employed with different traffic conditions, namely highly congested, congested, and uncongested. The MPRs of CAVs were defined based on previous studies (Olia et al., 2018; Talebpour and Mahmassani, 2016) with the judgement to cover a wide horizon of scenarios to consider various conditions. Olia et al. (2018) considered MPRs of $20 \%, 40 \%, 60 \%, 80 \%$, and 100\%, while Talebpour and Mahmassani (2016) adopted MPRs of $10 \%, 25 \%, 50 \%, 75 \%$, and $90 \%$. The emergence of CAVs will likely be gradual in reality and urges the need to examine low MPRs. High MPRs are taken into account to illustrate the magnitude of improvements associated with high MPRs of CAVs. The traffic indicators, in particular the throughput, TT, VKT, speed, density, and flow, are examined in Section 4.7.1. In addition, the environmental indicators include the GHG and NOx emissions, and their performance for different MPRs of CAVs and traffic conditions are discussed in Section 4.7.2.

\subsubsection{E2ECAV Traffic Analysis}

To account for stochasticity, five trials were conducted with different random seeds for every scenario as recommended by Law and McComas (1991). The traffic analysis was conducted at two levels: link and network. The most congested link in the network, which is on Front Street, was studied to further comprehend the changes of the traffic indicators with different settings. The link has two lanes with an $80 \mathrm{~km} / \mathrm{h}$ speed limit, while the downstream link has a single lane with a $30 \mathrm{~km} / \mathrm{h}$ speed limit. 


\section{Mean TT and VKT for Different MPRs of CAVs and Traffic Conditions}

Figure 4.7 shows that higher MPRs of CAVs offered significant reductions in the mean TT, but the reductions were at the cost of a slight increase in the mean VKT. The highly congested and congested traffic conditions benefited from the connectivity in E2ECAV distributed routing system much more than the uncongested traffic condition. For the highly congested and congested conditions, the decrease in the mean TT was due to the up-to-date information of the optimal routes shared with the CAVs. The evolution of the traffic condition was captured at every minute, and link cost was updated accordingly for a more efficient routing. If a vehicle takes a link of its optimal path, but the traffic condition changes on that path, a new route is estimated and provided at the nearest intersection. To minimize TT, vehicles were distributed in the network to use uncongested links regardless of distance as long as the TT cost was satisfied. Section 5.7.3 in Chapter 5 illustrates the longer travel distances when TT is the only routing objective.

On the other hand, when the traffic condition was uncongested, there was not a need for up-to-date information as the density (vehicle/kilometre) was very low and speed of the vehicles was almost equal to the speed limit. Vehicles did not have to avoid congested links as the network was lightly occupied, and this justified the negligible enhancement in terms of the mean TT.

When the MPR is $100 \%$ CAVs, the mean TT for the highly congested, congested and uncongested reduced by $41 \%, 32 \%$, and $10 \%$, respectively, compared to the base case of 100\% HDVs. The reduction in mean TT depends on the demand level. When the links were saturated, which is the the case for the highly congested or congested traffic conditions, the mean TT was enhanced by efficiently estimating the link cost and routing the vehicles.

For the highly congested traffic condition, the largest reduction in the mean TT of $37 \%$ was with a MPR of $30 \%$. When the MPR is $50 \%$ CAVs, the mean TT increased by $6 \%$ compared to $30 \% \mathrm{CAVs}$, and this can be due to re-routing. For $70 \%$ and $100 \% \mathrm{CAVs}$, the 
results were comparable for the mean TT. The mean TT for $100 \%$ CAVs was only $0.8 \%$ higher than $70 \%$ CAVs.

For the congested traffic condition, the mean TT reduces gradually with a higher percentage of CAVs without fluctuations compared to the highly congested traffic condition. Due to the demand level with the highly congested network, the route choice has a greater impact on the following vehicles compared to the congested traffic condition. The mean TT was almost the same for $50 \%, 70 \%$, or $100 \%$ of CAVs. Yet, the lowest mean TT is associated with $100 \%$ MPR of CAVs, and the mean TT for this case is $0.8 \%$ lower than $70 \%$ of CAVs.

Similar to the congested traffic condition, the uncongested traffic condition has the mean TT gradually reducing with a higher percentage of CAVs, but the change is almost insignificant.

A lower average TT was at the cost of a slight increase in the VKT. A proportional relationship exists between the mean VKT and MPR of CAVs. The increase in mean VKT occurs when a high MPRs of CAVs were employed. This is due to the fact that CAVs were directed to longer routes as long as the TT was minimized. As all of the possible routes in the network were examined at every updating interval, the outcome was an increase in travel distance in order to complete faster routes. The congested traffic condition has the highest mean VKT when there were $100 \%$ of CAVs, and the mean VKT for this case was $4 \%$ higher than the base case with $100 \%$ of HDVs. For the highly congested and uncongested traffic conditions, the mean VKT for $100 \%$ of CAVs increased by less than $2.5 \%$ compared to $100 \%$ of HDVs. Unlike the highly congested traffic network, the congested traffic condition had a larger number of options to choose for the routes. When the network is uncongested, the efficiency of the routing framework is not clear as there is not a need to reliably define the link cost. It is essential to note that the additional distance travelled may have an adverse impact on the environmental aspect, which is explored in Chapters 5 and 7. As shown in Figure 4.7, the best MPR of CAVs is $70 \%$ for the highly congested and uncongested conditions, but the best MPR of CAVs is $100 \%$ for congested traffic condition. The optimal MPR is a 
function of demand level, in which dynamics vary from a condition to another. When the network is highly congested, fewer route options are available compared to when the network is congested. Thus, a MPR of $100 \%$ CAVs was optimal for the congested network, while the highly congested network performs optimally with slightly less CAVs.

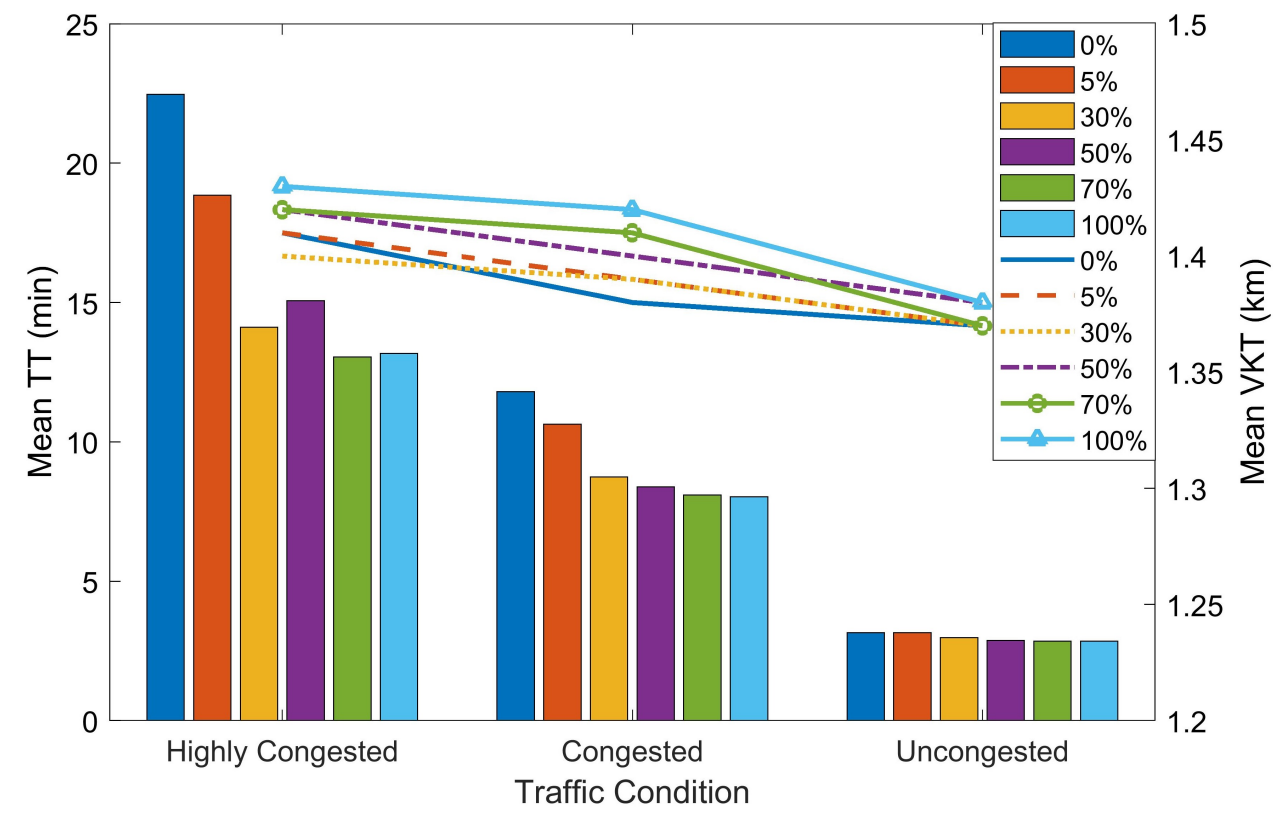

Figure 4.7 Mean TT (bars) and Mean VKT (lines) for Different MPRs of CAVs and Traffic Conditions

In summary, the efficiency of the proposed E2ECAV (Farooq and Djavadian, 2019) is the best when the traffic condition is congested or highly congested and when there are high MPRs of CAVs. When the network is saturated or close to being saturated, updating optimal routes in a timely manner is a major contributing factor to reduce the mean TT. When there were high MPRs of CAVs, the whole possible route options were examined for the best outcome. A lower mean TT was at the cost of a slightly higher mean VKT. Any perturbation or congestion was captured and routes were proactively updated while satisfying the routing objective. 


\section{Throughput of Different Traffic Conditions}

Figure 4.8a shows the throughput when the traffic condition is highly congested. There is a proportional relationship between the throughput and the MPR of CAVs. When 100\% CAVs is employed, it takes as much as $40 \%$ less time to load and unload the network compared to $0 \%$ CAVs.

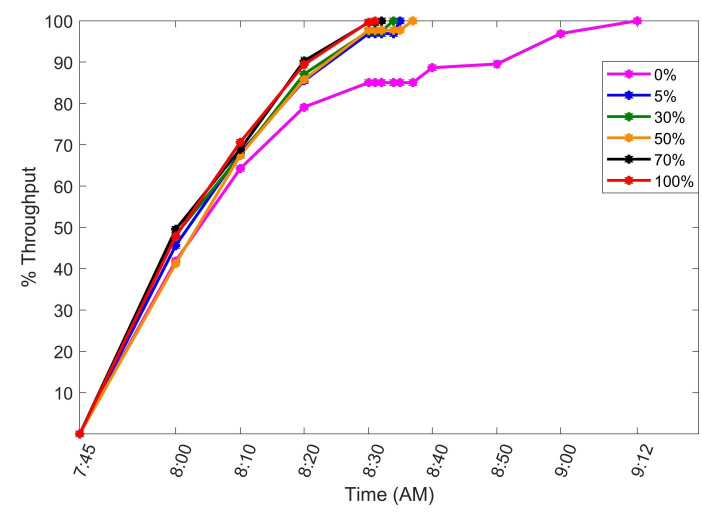

(a)

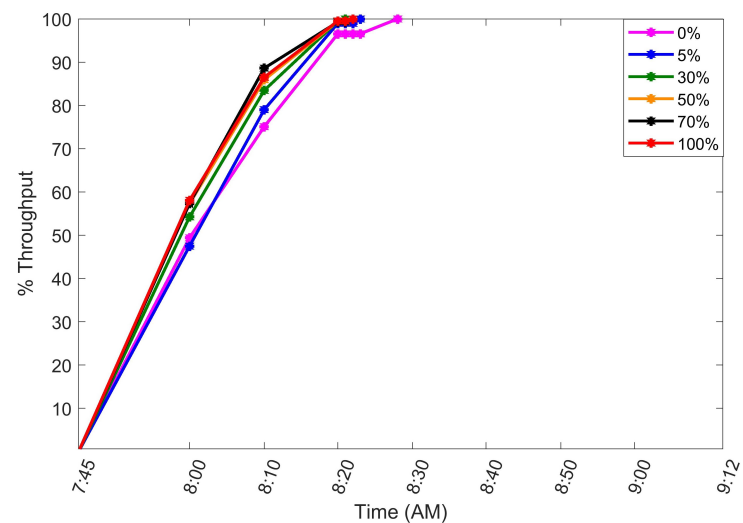

(b)

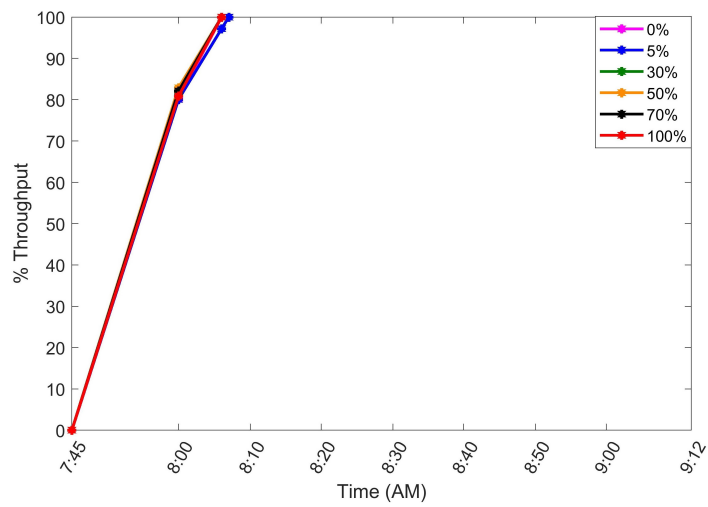

(c)

Figure 4.8 The Throughput for the a) Highly Congested, b) Congested, c) Uncongested Traffic Condition

The findings in this analysis were aligned with the results related to the mean TT. When there are $30 \%$ of CAVs, the network is loaded and unloaded quicker by 37 minutes compared to $100 \%$ HDVs. A longer mean TT occurred with 50\% CAVs compared to $30 \%$ CAVs as found in the previous section. In this analysis, 50\% CAVs requires three minutes of more 
time to empty the network compared to $30 \%$ CAVs. This can be due to additional re-routing when $50 \%$ CAVs is employed. A throughput of $100 \%$ was achieved at almost the same time for the $70 \%$ and $100 \%$ CAVs. The time vehicles spend in the network has a negative impact on the environment, and this aspect is discussed in detail in Chapters 5 and 7.

Figure $4.8 \mathrm{~b}$ shows the results for the congested traffic condition and demonstrates that a higher MPR of CAVs achieves 100\% throughput in less time. The variation between the scenarios of different MPRs is less compared to the case of highly congested traffic condition. While comparing the different traffic conditions, the difference in throughput for the same MPR is due to the demand level. This asserts that the efficiency of E2ECAV is higher when the network is more congested. The effect was more noticeable when reliable representative routes were provided to vehicles. When $5 \%$ CAVs was employed for congested traffic condition, the network was loaded and unloaded 5 minutes earlier compared to having 0\% CAVs. A MPR of 50\%, 70\%, and 100\% CAVs achieved 100\% throughput 6 minutes earlier than the case of $0 \%$ CAVs.

Finally, Figure 4.8c shows the throughput for the uncongested traffic condition and indicates that the improvement was negligible by adding more CAVs. These findings are aligned with the related results with the mean TT that were presented in the previous section.

\section{Fundamental Diagrams}

The fundamental diagrams illustrate the relationship between the three important traffic variables, namely speed, density, and flow (Papacostas and Prevedouros, 1993). The fundamental diagrams are supporting analyses at the link level. The most congested link in the network was on Front Street and was studied for this analysis. The link is of $80 \mathrm{~km} /$ hour speed limit and two lanes, while the downstream link is of $30 \mathrm{~km} /$ hour speed limit and one lane. The fundamental diagrams (Papacostas and Prevedouros, 1993) were developed to show the speed, density, and flow for the three traffic conditions: highly congested, congested, and uncongested. The MPRs of CAVs at 0\%, 5\%,30\%, 50\%, 70\%, and 100\% were 
incorporated into this analysis with five trials for every scenario, and the range of MPR of CAVs allow the magnitude of improvement of employing different MPRs of CAVs to be seen with the fundamental traffic variables.

Figure 4.9a shows the results of Speed-Density for the highly congested traffic condition. A proportional relationship exists between the speed and MPR of CAVs. When high MPRs of CAVs were employed, the vehicles averted congested links and traveled on links with a higher speed limit and longer distance in order to minimize the travel time. These results are similar to those found in Section 4.7.1. The trade off is demonstrated in detail between TT and the VKT in Section 5.7.3. The speed increased by as much as $30 \mathrm{~km} / \mathrm{h}$ for $100 \%$ CAVs compared to 0\% CAVs. Figure 4.9a also shows that a higher MPRs of CAVs improves the density of vehicles within the link. This is due to the adopted short spacing distance of 1 meter and reaction time of 1 second by CAVs. Furthermore, as the optimal routes were updated based on the most up to date traffic conditions, vehicles avoided congested links when they do not satisfy the optimization objective of routing as shown in Equation 2.1. For the $100 \%$ CAVs scenario, the density almost reaches the jam density of the link of 166 vehicle/kilometre/lane. This means more vehicles were accommodated on the link. The reduced reaction time and safe spacing distance of CAVs compared to HDs improved the density and also introduced enhancements in the capacity on the link as shown in Figures $4.9 \mathrm{~b}$ and $4.9 \mathrm{c}$. It is worth mentioning that the three fundamental variables, namely speed, density, and flow, depend on each other. When one of them changed, the other two were modified (Papacostas and Prevedouros, 1993). The increased link capacity supports the findings in Sections 4.7.1. When 100\% CAVs is used, the density and flow have increased by $65 \%$ and $133 \%$, respectively, compared to the base case of $0 \%$ CAVs.

When the traffic condition is congested as shown in Figure 4.10, the same trend is demonstrated compared to when the traffic condition is highly congested. Speed, density, and flow were improved with higher MPRs of CAVs. However, the variance in value of the three traffic fundamental variables among the range of MPRs of CAVs is less compared to the highly 


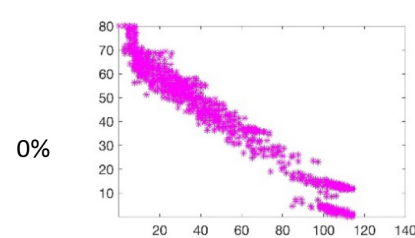

(a)

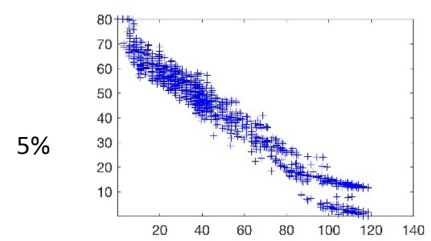

(a)

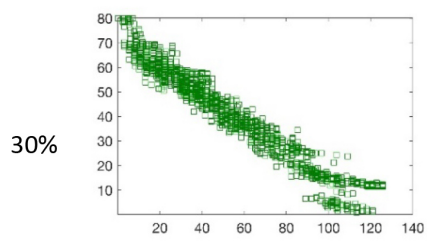

(a)

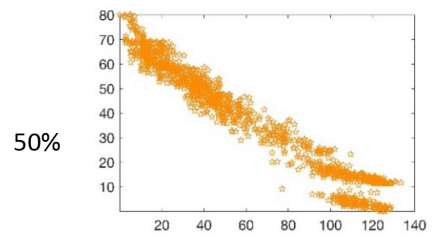

(a)

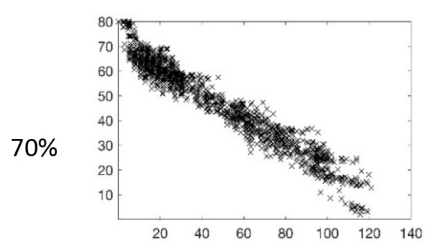

(a)

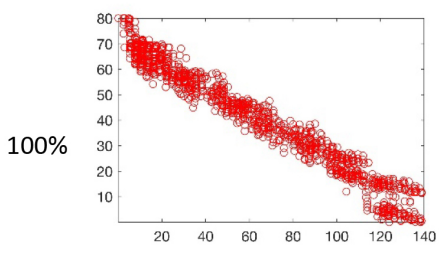

(a)

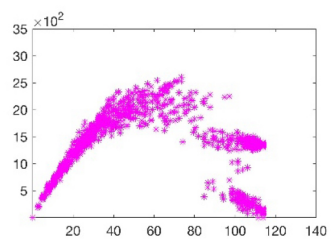

(b)

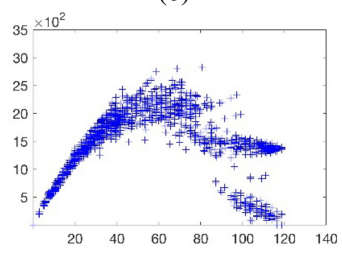

(b)

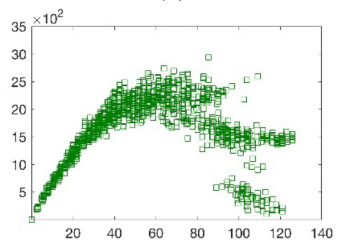

(b)

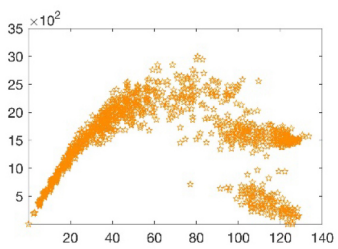

(b)

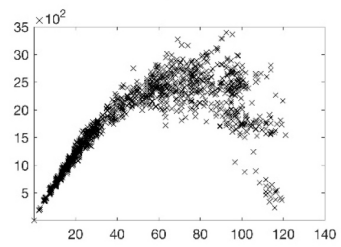

(b)

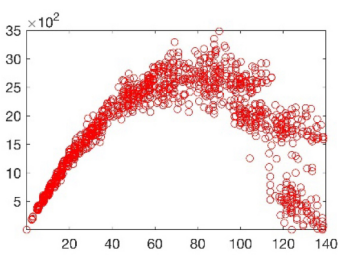

(b)

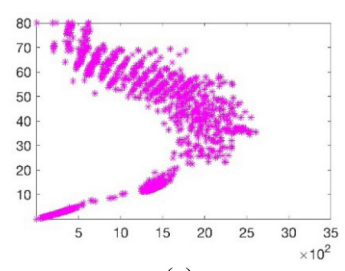

(c)

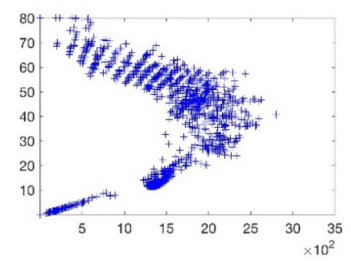

(c)

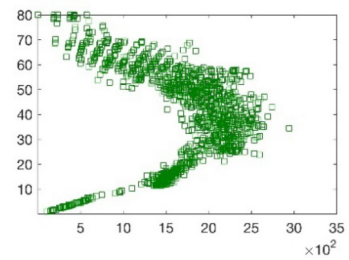

(c)

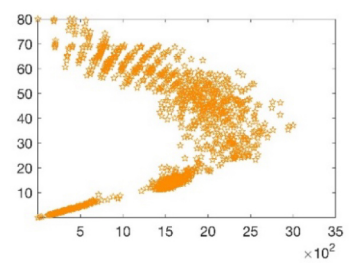

(c)

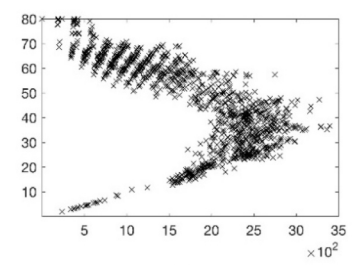

(c)

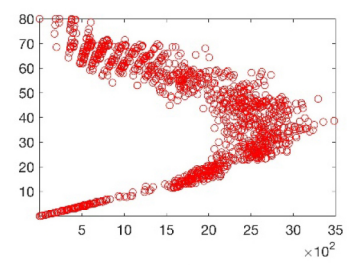

(c)

Figure 4.9 Fundamental Diagrams of a) Speed $(\mathrm{km} / \mathrm{h})$ vs. Density (veh/ $\mathrm{km} / \mathrm{ln})$, b) Flow $(\mathrm{veh} / \mathrm{h})$ vs. Speed $(\mathrm{km} / \mathrm{h})$, and c) Flow (veh/h) vs. Density (veh $/ \mathrm{km} / \mathrm{ln}$ ) for the Most Congested Link under the Highly Congested Traffic Condition and Different MPRs of CAVs 


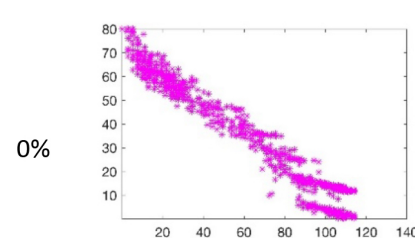

(a)

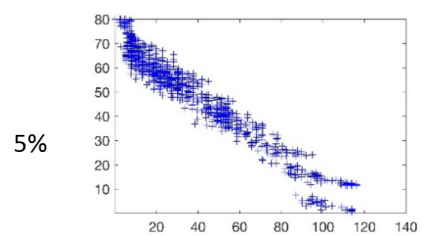

(a)

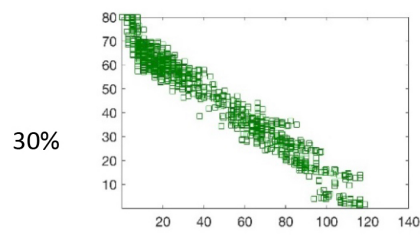

(a)

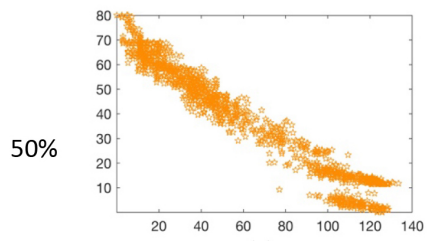

(a)

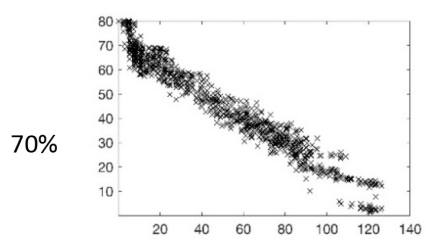

(a)

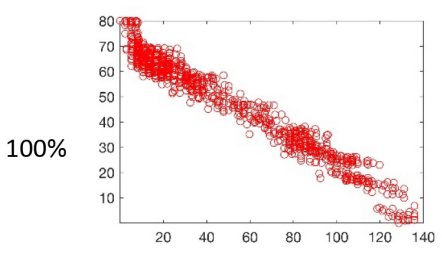

(a)

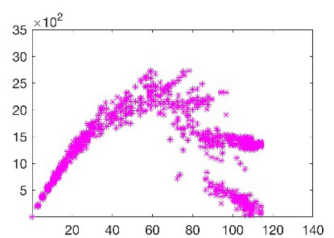

(b)

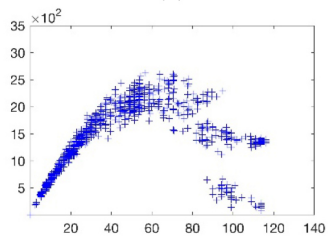

(b)

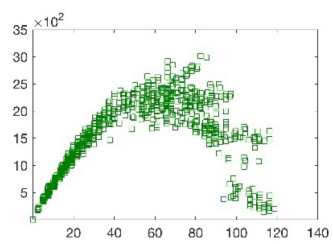

(b)

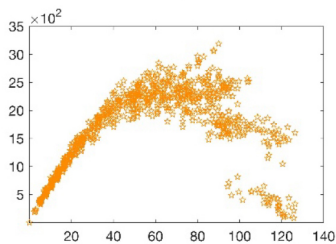

(b)

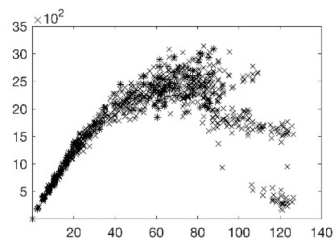

(b)

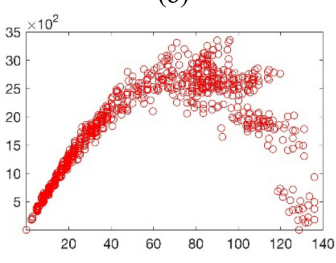

(b)

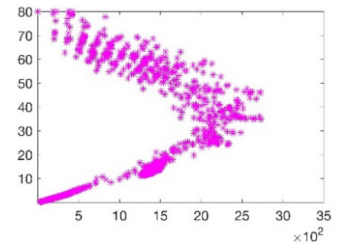

(c)

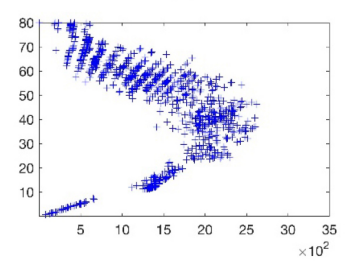

(c)

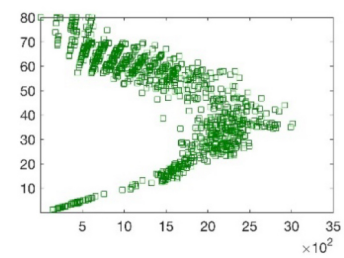

(c)

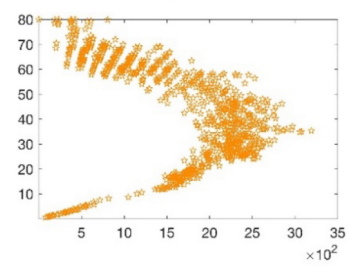

(c)

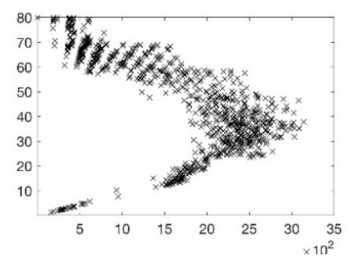

(c)

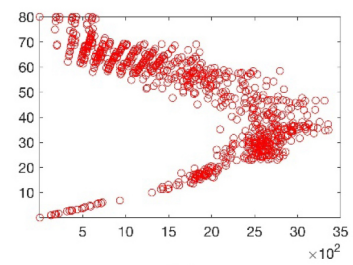

(c)

Figure 4.10 Fundamental Diagrams of a) Speed $(\mathrm{km} / \mathrm{h})$ vs. Density $(\mathrm{veh} / \mathrm{km} / \mathrm{ln})$, b) Flow $(\mathrm{veh} / \mathrm{h})$ vs. Speed $(\mathrm{km} / \mathrm{h})$, and c) Flow (veh/h) vs. Density $(\mathrm{veh} / \mathrm{km} / \mathrm{ln})$ for the Most Congested Link under the Congested Traffic Condition and Different MPRs of CAVs 


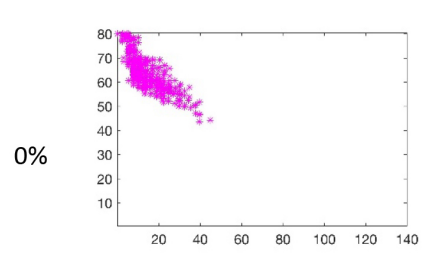

(a)

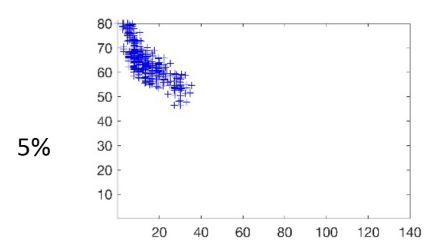

(a)

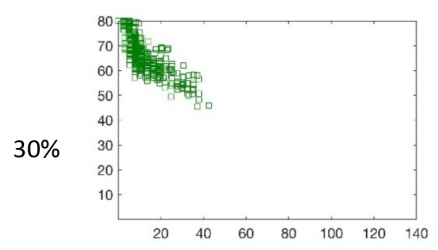

(a)

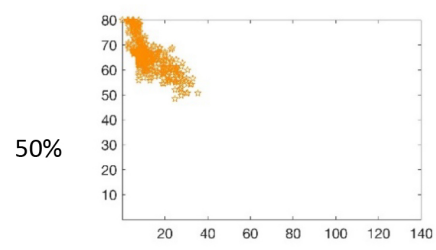

(a)

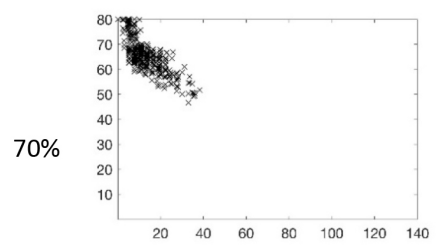

(a)

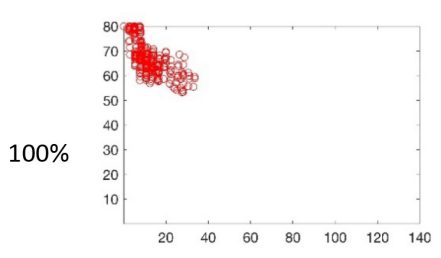

(a)

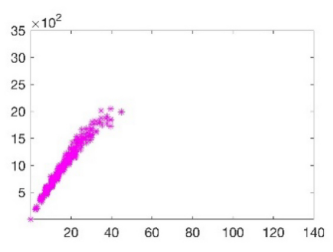

(b)

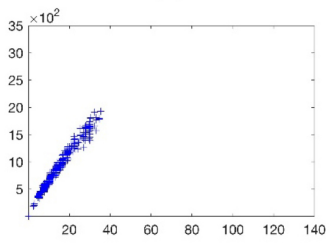

(b)

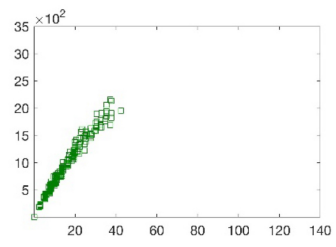

(b)

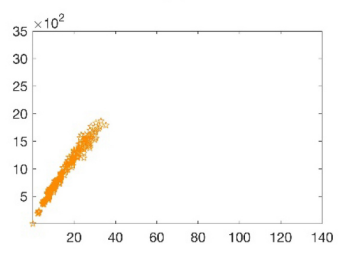

(b)

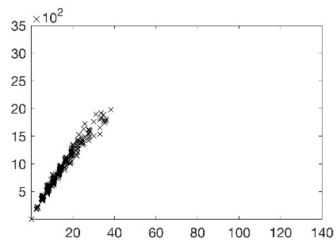

(b)

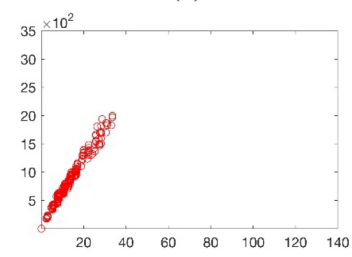

(b)

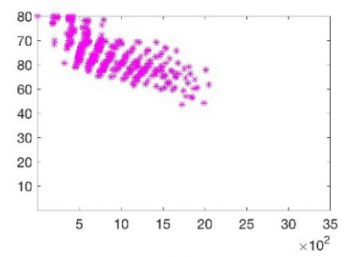

(c)

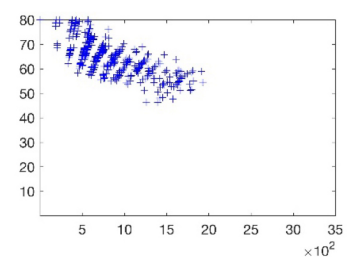

(c)

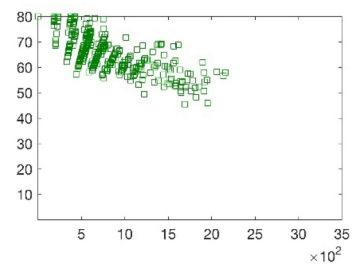

(c)

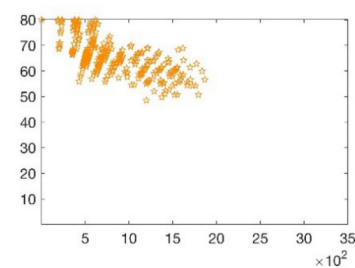

(c)

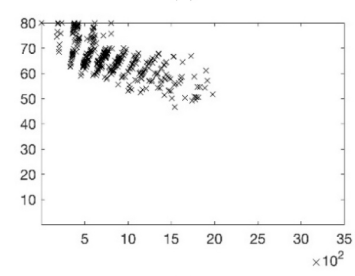

(c)

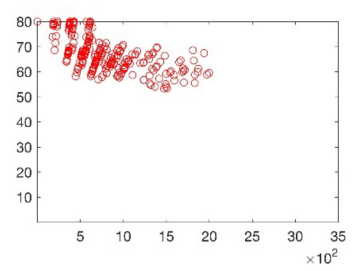

(c)

Figure 4.11 Fundamental Diagrams of a) Speed $(\mathrm{km} / \mathrm{h})$ vs. Density $(\mathrm{veh} / \mathrm{km} / \mathrm{ln})$, b) Flow (veh/h) vs. Speed $(\mathrm{km} / \mathrm{h})$, and c) Flow (veh/h) vs. Density $(\mathrm{veh} / \mathrm{km} / \mathrm{ln})$ for the Most Congested Link under the Uncongested Traffic Condition and Different MPRs of CAVs 
congested traffic condition. This occurred due to the demand level.

Finally, when the traffic condition is uncongested as shown in Figure 4.11, the enhancement in the speed, density, and flow is low as the links, in general, are unoccupied. This means that speed of the vehicles is almost equal to the speed limit, and the density is very low as shown in Figure 4.11a.

The findings of this analysis support the results from the previous analysis. Also, when the network is highly congested or congested, there is a strong necessity to proactively estimate the link cost and to update the route of the vehicles accordingly.

\section{Speed, Density, and Flow Over Time of Different Traffic Conditions}

This analysis was conducted at the link level using the most congested link in the network, which is on Front Street. The three fundamental traffic indicators, namely speed, density, and flow, were analyzed over time to better observe the dynamics at a more disaggregated level. Figure 4.12a shows that the link was loaded and unloaded quicker with higher MPRs. With 100\% CAVs, the link was loaded and unloaded in 54\% less time compared to 100\% HDVs. With a higher MPR of CAVs, the speed increased over time. The speed was as much as $30 \mathrm{~km} / \mathrm{h}$ higher with $100 \% \mathrm{CAVs}$ than with 0\% CAVs. At the beginning of the simulation, the speed from 7:45am to 8:00am was significantly higher when the MPR is higher or equal to $50 \%$. The justification is due to the dynamic proactive nature of the updating process for the link cost. When low MPRs were used and the link was congested, vehicles were still sent to the link, making the traffic situation worse on the link. On the other hand, with higher MPRs, vehicles were distributed in the network and were not sent to the most congested link unless the routing objective was satisfied. During the peak period from 8:00am to 8:20am, the difference in speed over time is clear between the different MPRs of CAVs. During the peak period, the speed on the studied link was greater when more CAVs were deployed.

While examining the density over time in Figure 4.12b for the highly congested traffic condition, the use of a shorter safe spacing distance and reaction time contributes to a higher 


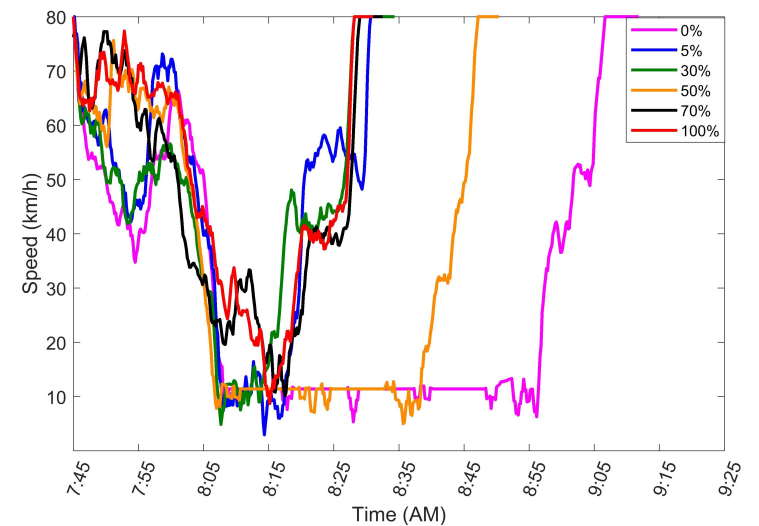

(a)

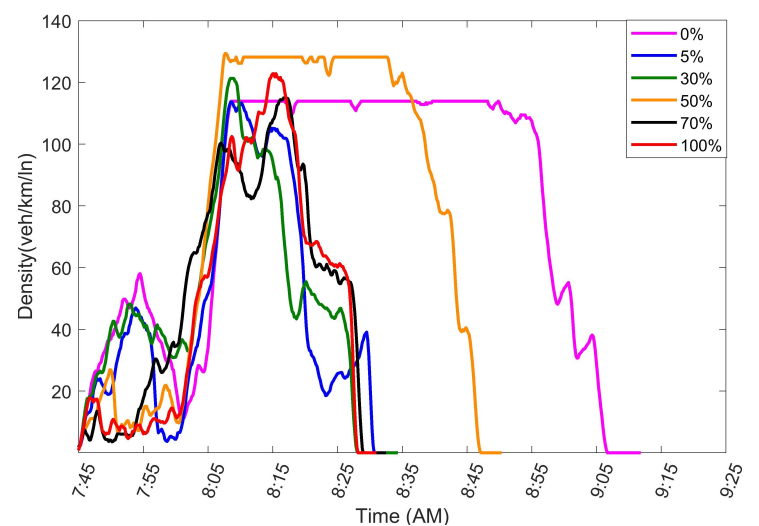

(b)

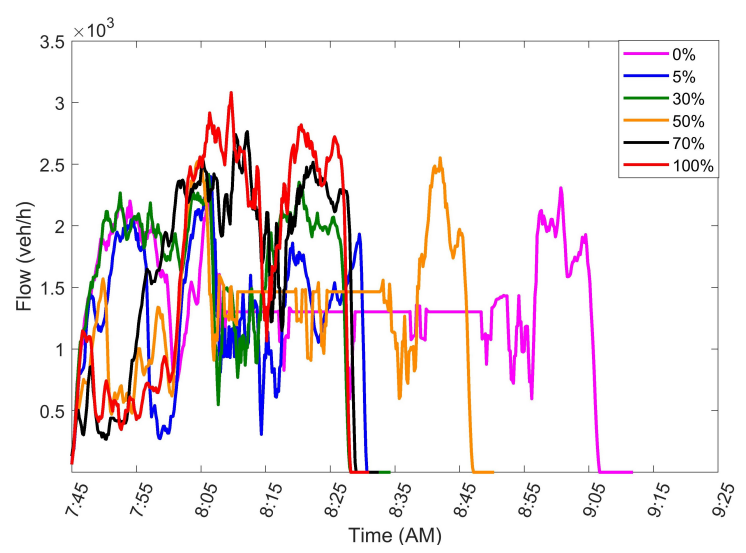

(c)

Figure 4.12 The Most Congested Link in the Network under the Highly Congested Traffic Condition and its a) Speed, b) Density, c) Flow

density with higher MPRs during the peak period from 8:00am to 8:20am. In general, more vehicles were accommodated on the links when a higher MPRs was employed. At a point of time, the density on the link was three times more when $100 \%$ CAVs is employed compared to $30 \%$ CAVs. Based on the period between the beginning of the simulation at 7:45am to 8:00am, the density was low on the most congested link in the network if the MPR of CAVs was greater than or equal to $50 \%$. During this period, this link does not satisfy the optimization objective, and it is averted by the vehicles as a result. However, when the demand is completely loaded into the network from 8:00am to 8:20am, the whole network is congested, and this link is used as long as it contributes to minimizing the TT cost. 


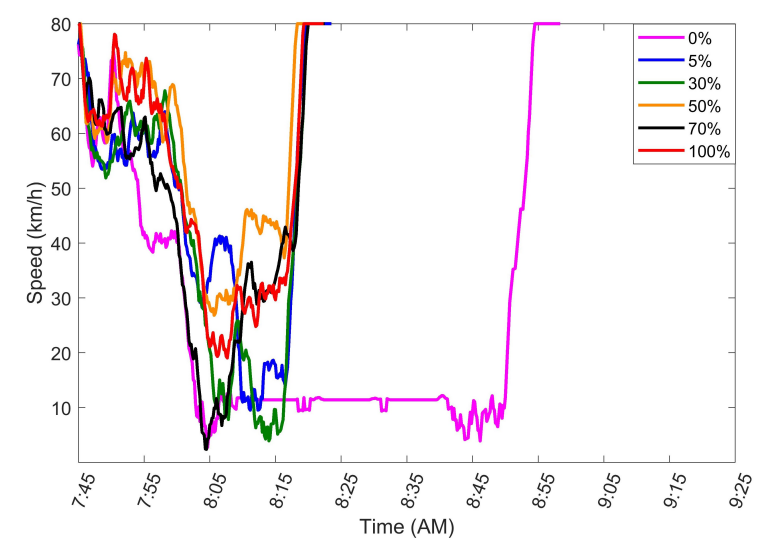

(a)

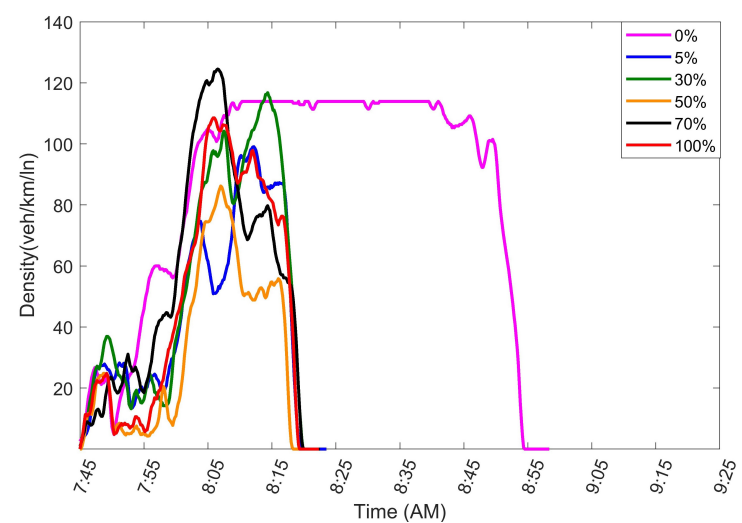

(b)

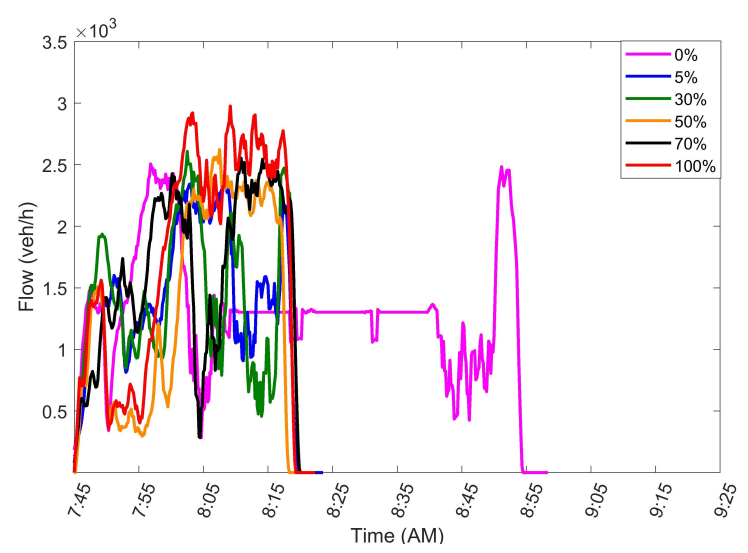

(c)

Figure 4.13 The Most Congested Link in the Network under the Congested Traffic Condition and its a) Speed, b) Density, c) Flow

The three fundamental traffic variables are correlated (Papacostas and Prevedouros, 1993), and this correlation explains the increase in the traffic flow on the studied link with higher MPRs in Figure 4.12c. A supporting trend is captured in this figure that illustrates the high flow when MPRs are less than 50\% during the period of 7:45am and 8:00am. During this period, the density is high, and the speed is low. The relationship between speed and density triggers the increase in flow. For the peak period from 8:00am and 8:20am, the flow is higher for MPRs equal to or more than $50 \%$.

Figure 4.13 shows the results from the congested traffic condition and demonstrates the same trend that was observed in Figure 4.12 with the highly congested traffic condition. 


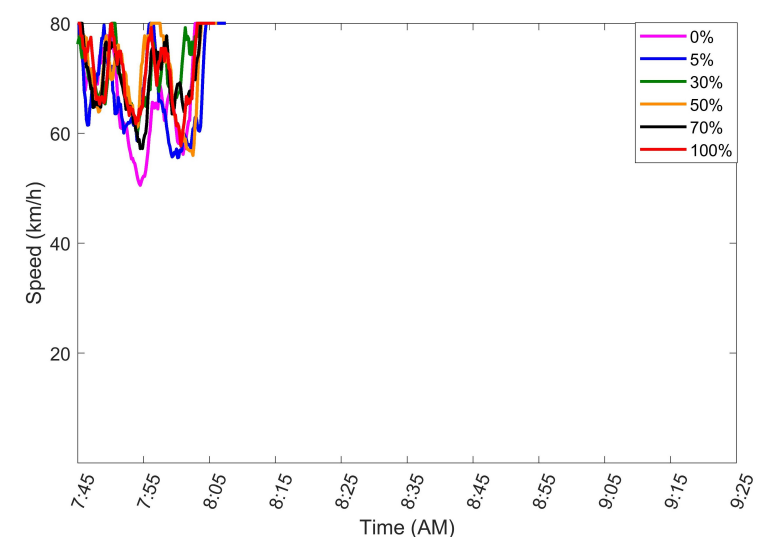

(a)

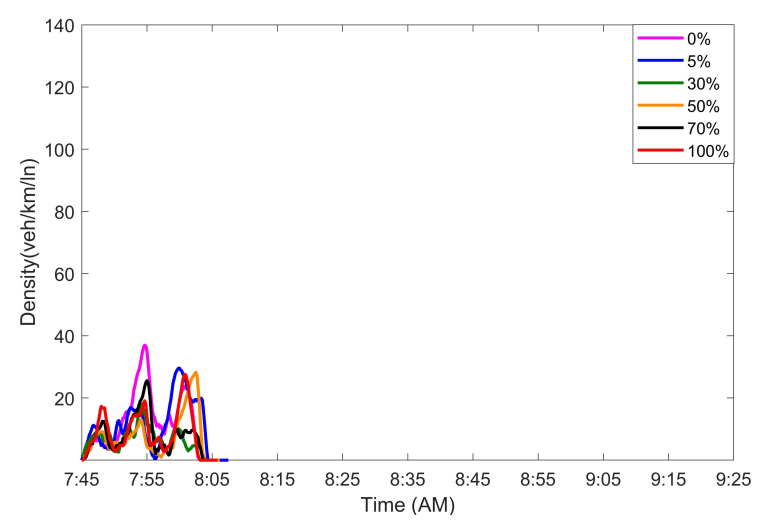

(b)

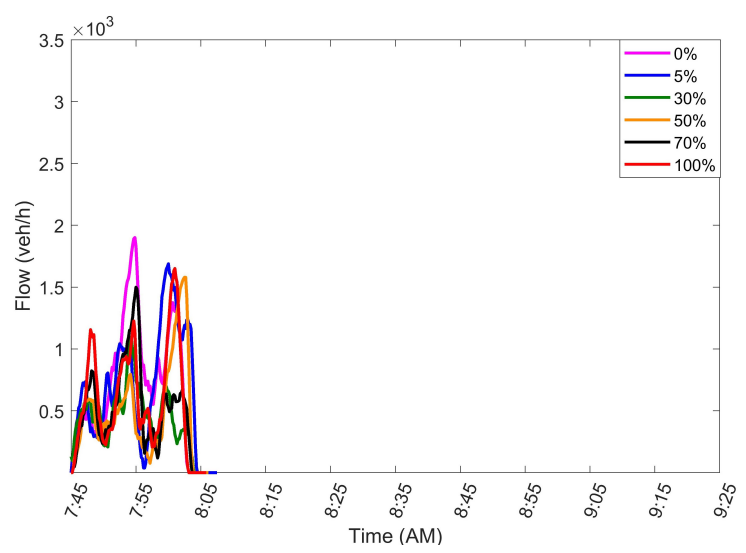

(c)

Figure 4.14 The Most Congested Link in the Network under the Uncongested Traffic Condition and its a) Speed, b) Density, c) Flow

Nevertheless, with less congestion, the network has been loaded and unloaded faster for the same scenario. This event occurs due to the demand level and number of vehicles in the network. Due to the demand level, the congested traffic condition has less variation compared to the highly congested condition in the performance indicators between the different MPRs.

Finally, Figure 4.14 shows there is not much value in employing E2ECAV when the network is uncongested because the link is almost at the speed limit and the density is very low. 


\subsubsection{E2ECAV Environmental Analysis}

It is paramount to assess the impact of employing any of ICT forms, such as intelligent vehicles and sensing technology, on the environment. Greenhouse gases are the gases that trap heat in the atmosphere (EPA, 2020a). GHG is the major factor that is negatively contributing to climate change (Z. Liu et al., 2020). Air pollutant NOx, which reflects on the public health aspect, represents a family of seven compounds (Cox, 1999; Price et al., 1997). Figures 4.15a and 4.15b, respectively, show the total produced GHG and NOx emissions for different MPRs of CAVs and traffic conditions. Figure 4.15a shows that the effect of changing the MPR of the CAVs was more significant when the traffic condition was highly congested or congested. This aligns with the findings in the previous section.

For the highly congested traffic condition, Figure 4.15a shows that the total GHG emissions reduced by $35 \%$ for 30\% CAVs compared to 0\% CAVs. Between $30 \%$ and $100 \%$ CAVs, a fluctuation has been noticed in the total GHG. An increase in the total emitted GHG occurred for 50\% MPRs of CAVs compared to 30\% CAVs. As shown in Figure 4.7.1, this was triggered by the additional TT and VKT spent in the network. For $50 \% \mathrm{CAVs}$, the additional re-routing could be the trigger for the deterioration. Among the MPR of CAVs, $70 \%$ MPR of CAVs had the lowest total GHG that was $41 \%$ lower compared to $100 \%$ HDVs.

When the network was congested, the GHG emissions reduced gradually without fluctuations as the MPR of CAVs increased. More route options were available for the congested network compared to the highly congested network. This justifies the fewer fluctuations of total GHG emissions when the network was congested compared to the highly congested network. For the congested network with a 30\% MPR of CAVs, the total produced GHG was significantly lower by $23 \%$ compared to the $0 \%$ MPR of CAVs. Due to the variation in the demand level, the variance in GHG emissions between the different MPRs was smaller compared to the highly congested traffic condition. While comparing $100 \%$ CAVs to $0 \%$ CAVs, the former reduced the total GHG emissions by $28 \%$ for the congested network. The 
emissions were reduced further by $39 \%$ for the highly congested traffic condition.

The impact of the MPRs of CAVs was negligible when the network was uncongested. This result is also supported by the outcomes from the previous sections in this study.

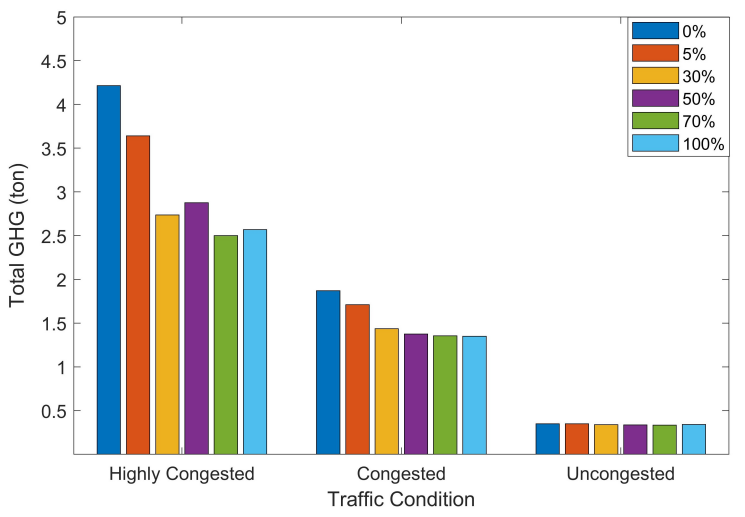

(a)

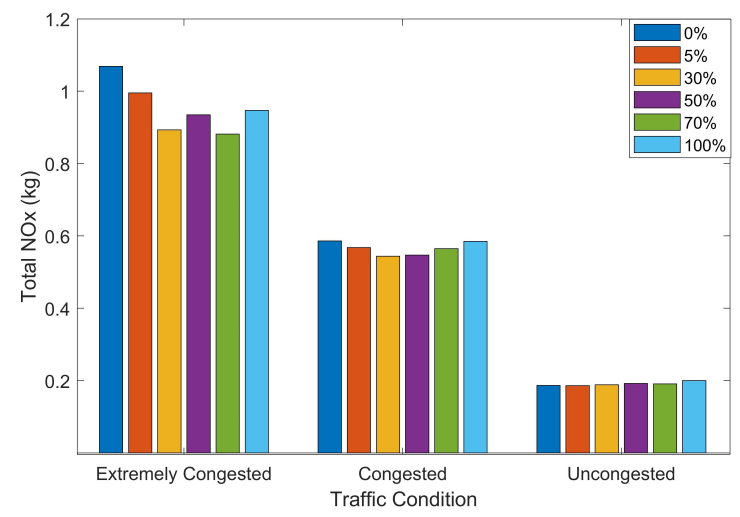

(b)

Figure 4.15 The Impact of Different MPRs of CAVs and Traffic Conditions on the a) Total GHG and b) Total NOx

High MPRs of CAVs contribute to a lower mean TT, and this results in less total GHG emissions because the network was generally loaded and unloaded quicker. It can be concluded that higher MPRs of CAVs will result in less GHG emissions. It is crucial to note that the optimal MPR of CAVs differs depending on the traffic condition. For the highly congested and uncongested condition, the optimal MPR was 70\%, and the total GHG was reducted by $41 \%$ and $5 \%$, respectively. The optimal MPR was $100 \%$ for the congested traffic condition, and it reduced the GHG emissions by $28 \%$. The difference between $100 \%$ and $70 \%$ CAVs for the produced GHG is marginal among the three traffic conditions (Tu, Alfaseeh, et al., 2019). It is important to note that in Figure 4.15a the trend is very similar to trend with the mean TT in Figure 4.7.1 for the three traffic conditions. This illustrates the explicit impact of the time spent in the network on the total GHG emissions. A longer travel time by the vehicles in the network results in more GHG emissions.

With respect to the total NOx emissions, the trends are not similar to those for the GHG emissions. Higher MPRs do not always decrease the total NOx. The major justification is 
the relationship between NOx and speed, which is quasi-convex (Djavadian, Tu, et al., 2020). It has also been found that there is a proportional relationship between NOx and aggressive driving (Tu, Alfaseeh, et al., 2019). With higher speeds, more NOx is generally emitted. Figure 4.15b demonstrates that there are a lot of fluctuations in the total NOx for the highly congested and congested traffic conditions, and this can again be linked to the speed on the links. For the highly congested traffic condition, 70\% CAVs is the optimal MPR that reduces the emissions by $18 \%$ compared to $0 \%$ CAVs. For the congested traffic condition, the $100 \%$ CAVs scenario produces as much NOx as 0\% CAVs. When the network is uncongested, the total NOx increases, starting from 5\% CAVs. For the uncongested network, 100\% CAVs increased the NOx emissions by $7 \%$ compared to $0 \%$ CAVs.

\subsection{Concluding Remarks}

Both the traffic and environmental indicators were enhanced by exploiting the advancements of ICT. ITS, which uses ICT developments, was proposed as the most favourable tool to reduce the undesirable impact of congestion on the traffic and environmental aspects. In this chapter, a dynamic distributed routing framework, E2ECAV (Farooq and Djavadian, 2019), was assessed in detail, and its application for eco-routing was investigated. It has been found that higher MPRs of CAVs offer better traffic and environmental characteristics in general based on the flow, speed, density, and GHG emissions. The mean TT decreased when higher MPRs of CAVs were employed, but the mean VKT increased slightly at the same time. For $100 \%$ CAVs, the mean TT decreased by $41 \%$ when the network was highly congested compared to $0 \%$ CAVs. Higher MPRs of CAVs contributed to a higher density during the peak period on links due to the shorter safe spacing distance and reaction time. As a result, more vehicles were accommodated on links. When the traffic condition was highly congested, the speed at the link level was as much as $30 \mathrm{~km} / \mathrm{h}$ higher for 100\% CAVs compared to $100 \%$ HDVs. For the highly congested and congested traffic conditions, significant improvements 
were noticed compared to the uncongested traffic condition. The efficiency of E2ECAV was not completely exploited when the network was uncongested, and the links were lightly used. When they were congested, the improvements in the traffic and environmental characteristics resulted due to properly estimating the link cost and updating it in a timely manner. From the environmental perspective, the major findings are as follows. In general, higher MPR of CAVs provided lower total GHG emissions. The optimal MPR varied for the different traffic conditions. The optimal MPR was $70 \%$ for the highly congested and uncongested traffic conditions with a reduction in the total $\mathrm{GHG}$ by $41 \%$ and $5 \%$, respectively. For the congested traffic condition, 100\% CAVs was the optimal MPR, and it reduced the total GHG by $28 \%$ compared to the base case. The trend observed by the total GHG emissions was comparable to the trend with the mean TT. This shows a direct relationship between the time spent in the network and the emitted GHG. The total NOx emissions follows a different trend compared to the GHG due to the relationship between speed and emitted NOx.

In summary, both the traffic and environmental characteristics have improved substantially from utilizing the CAVs in a distributed routing framework. This shows that connectivity is a beneficial feature that is capable of sharing the real-time status of a network to offer proactive routing. The examined E2ECAV adopted the FIFO logic and applied the lateral movement only at the beginning of the links. Future applications are urged to address these aforementioned two aspects. As E2ECAV proved its ability to reliably capture the changes in traffic, it contributed to improvements in the traffic and environmental characteristics of a network. E2ECAV is a good candidate to be deployed for eco-routing application that considers the environmental dimension. In the next chapter, Chapter 5, myopic multi-objective routing was applied while employing the efficient E2ECAV dynamic distributed routing system to mitigate the undesirable impact of transportation systems on the environment. Furthermore, anticipatory multi-objective eco-routing was applied in Chapter 7 to further improve the results while using the E2ECAV. 


\section{Chapter Five}

\section{Myopic Eco-Routing}

This chapter has resulted in the following publications:

1. Alfaseeh, Lama, Shadi Djavadian, Ran Tu, Bilal Farooq, \& Marianne Hatzopoulou (2019). "Multi-Objective Eco-Routing in a Distributed Routing Framework". In 2019 IEEE International Smart Cities Conference (ISC2) (pp. 747-752). IEEE.

2. Djavadian, Shadi, Alfaseeh, Lama, Tu, Ran, Farooq, Bilal, \& Hatzopoulou Marianne (2020). "Multi-Objective Eco-Routing in a Distributed Traffic Management Framework with a Case Study of Downtown Toronto". In $99^{\text {th }}$ Annual Meeting of Transportation Research Board, Washington, DC, January 2020. 


\subsection{Introduction}

It was estimated by the Council of Ministers Transportation and Highway Safety (2012) that the Greater Toronto Area (GTA) contributed to $42.5 \%$ of the total congestion cost in Canada in 2006. Among the proposed solutions, routing has been considered as a tool to alleviate congestion in transportation systems. Myopic routing, which is most commonly used, considers only travel time as the routing objective (J. Sun and H. X. Liu, 2015; Rakha, Ahn, and Moran, 2012). In addition, a predominantly centralized routing framework has been utilized (J. Sun and H. X. Liu, 2015; Rakha, Ahn, and Moran, 2012) despite the advancements in ICT. Based on the aforementioned shortcomings of the existing routing applications and the advancements in ICT, there is a strong necessity to extend the routing concept and incorporate the environmental dimension and optimize more than one objective by applying multi-objective eco-routing in a distributed routing framework. This application demonstrates the potential effect of optimizing more than one objective simultaneously while the environmental dimension is considered. Myopic routing depends on previous or current traffic and/or environmental states. This makes myopic routing easy to apply compared to the anticipatory routing that requires predictive models.

In this chapter, single and multi-objective myopic eco-routing strategies were examined while adopting the efficient distributed routing framework, E2ECAV, which was examined in Chapter 4. The impact of the myopic routing strategies was examined from the traffic and environmental perspectives. The performance indicators included, average travel time (TT), average vehicle kilometres travelled (VKT), total produced greenhouse gas (GHG) emissions, and total produced nitrogen oxide $(\mathrm{NOx})$. Network level analysis in addition to the path analysis of a randomly chosen vehicle were conducted to support the major findings. The network level analysis showed the global impact of different routing strategies, while the path analysis illustrated how the paths vary based on a routing objective. The myopic routing strategies were the base for comparison when anticipatory routing strategies were applied in 


\section{Chapter 7 .}

Figure 5.1 illustrates the chapter framework and its relationship with the other chapters in this dissertation. This chapter is organized as follows. Section 5.2 presents a brief literature related to the eco-routing studies and the drawbacks associated with them. Section 5.3 illustrates the specifications of the adopted traffic and emission models in this chapter. Section 5.4 demonstrates the characteristics of the case study employed for the myopic routing application. Section 5.5 introduces the investigated GHG costing approaches to define the best one for the setting of this dissertation in terms of the spatial and temporal level of resolution. Section 5.6 includes the details related to the assessed routing strategies in this chapter. Section 5.7 presents the analysis components related to the GHG costing approaches followed by the myopic routing strategies. Finally, Section 5.8 summarizes the major findings of this chapter.

\subsection{Literature}

Based on the comprehensive literature review of the eco-routing studies in Chapter 2, the crucial findings are as follows. It was found that the GHG emission cost was not defined in detail. Every application is associated with specifications related to different aspects, such as the level of resolution of the data, scale of the case study, and purpose of the application. That is, defining the GHG costing approach in a detailed manner helps practitioners to decide what GHG costing approach is the best for their setting. In some cases, the GHG emission was based on either spent travel time or travelled distance. In both cases one dimension was missing, either spatial or temporal (Alfaseeh and Farooq, 2020b). For a real-time application at a disaggregated level, namely routing, incorporating the spatial and temporal dimensions reflects more reliably on the traffic conditions.

With respect to the aggregation level of the traffic and emission models while ecorouting was applied, macroscopic and mesoscopic models were dominating (Luo et al., 2016; 


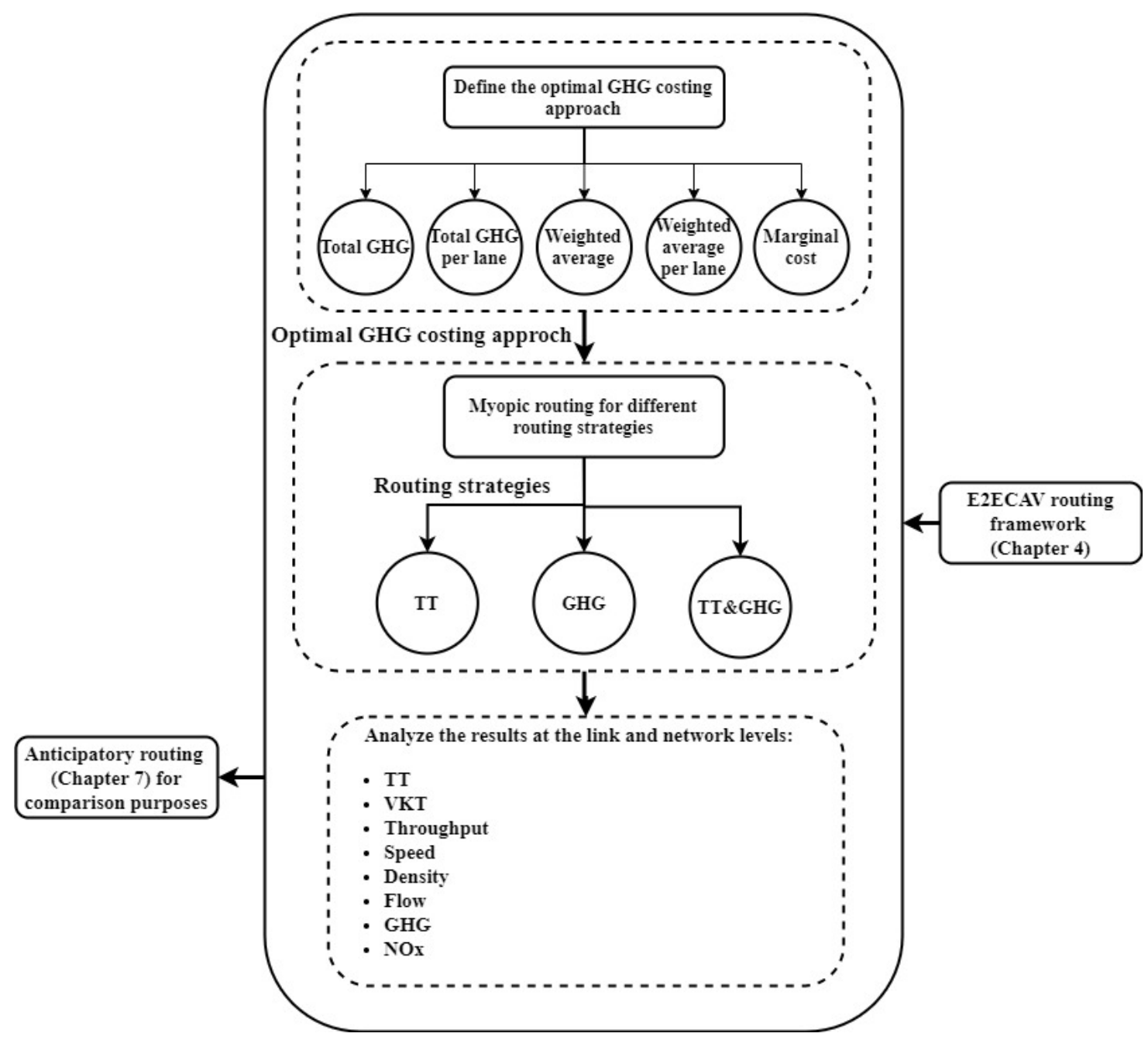

Figure 5.1 Chapter Five Framework

Patil, 2016; Benedek and Rilett, 1998), despite the dramatic advancement in ICT. The low level of resolution of data points is not suitable for real-time routing applications (Alfaseeh, Djavadian, Tu, et al., 2019). As speed is the most important variable to estimate the GHG emissions, using average speed contributed to emission underestimation. This also means that congestion on links was not captured in a timely manner (Long et al., 2016; Patil, 2016; Guo, S. Huang, and Sadek, 2013; Aziz and Ukkusuri, 2012).

In terms of scaleability, case studies were limited and restrictions were made on OD pairs, especially when a microscopic level of aggregation of traffic and emission models was 
employed due to the complexity (Luo et al., 2016; Long et al., 2016; J. Sun and H. X. Liu, 2015). Small case studies refer to a segment on a road, part of a highway, or a zone with limited number of intersections. In some cases, only one destination was adopted (Long et al., 2016). Utilizing small case studies means that a model is not scaleable and does not reflect on a realistic situation.

Lastly, one routing objective was optimized at a time, travel time, distance, or emissions (J. Sun and H. X. Liu, 2015; Guo, S. Huang, and Sadek, 2013; Andersen et al., 2013; Benedek and Rilett, 1998). Routing objectives may conflict, which makes multi-objective routing significantly more complicated compared to single objective routing. For instance, increasing throughput, which can be achieved by choosing higher speed links, may have an adverse impact on produced GHG due to the quasi-convex relationship between speed and GHG (Djavadian, Tu, et al., 2020). Increasing distance travelled while minimizing travel time may also contribute to more produced GHG and NOx emissions (Alfaseeh and Farooq, 2020a).

Thus, this chapter overcomes the aforementioned shortcomings of previous work. First, several GHG costing approaches were examined and the best one was adopted throughout this dissertation when required. Myopic single- and multi-objective eco-routing strategies were applied in the efficient distributed routing framework, E2ECAV (Farooq and Djavadian, 2019). A microscopic level of aggregation of the traffic and emission models was used to assure a high level of resolution of data points. Downtown Toronto was the case study, which experiences recurrent congestion during the morning peak hour. The outcome of myopic routing application in this chapter was used as the benchmark for the comparison with the outcome of the anticipatory routing application in Chapter 7. Djavadian, Tu, et al. (2020) applied myopic multi-objective eco-routing in a similar setting to this chapter, but while considering the cost of idling as a penalty at downstream intersection of a studied link at every interval. Four main scenarios were analyzed, travel time with $100 \%$ HDVs, travel time with 100\% CAVs, GHG with 100\% CAVs, travel time and idling penalty with 100\% 
CAVs, and travel time including idling penalty cost and GHG with 100\% CAVs. They found that including penalty cost contributed to slight reductions in average travel time and total produced GHG (Djavadian, Tu, et al., 2020).

\subsection{Traffic and Emission Models}

An agent-based traffic and emission simulation has been adopted in this part of the dissertation to synthesize GHG and traffic characteristics for links at a very high temporal resolution (Djavadian and Farooq, 2018). As explained in Chapter 3, the agent-based simulator em-

ployed the calibrated Intelligent Driver Model (IDM) (Treiber, Hennecke, and Helbing, 2000) for the vehicular movement. The second-by-second vehicular dynamics were captured and the cost of the links was estimated accordingly as presented in detail in Chapter 3 . The link cost was updated at every minute as found in Alfaseeh, Djavadian, and Farooq (2018). Travel demand was obtained from the Transportation Tomorrow Survey (TTS) (DMG, 2011). The time dependent exogenous demand Origin-Destination (OD) matrices were based on 5 minute intervals from TTS. The demand of 2014 was obtained from the data provided by TTS while using the proper growth factor, that represents congested traffic condition, in this chapter. Simulation ended once all of the vehicles reached their destinations.

With reference to the emission model, MOVES was the deployed emission model to generate the second-by-second GHG (in $\mathrm{CO}_{2 \mathrm{eq}} \mathrm{g} / \mathrm{sec}$ ) emissions of every vehicle (USEPA, 2015). The second-by-second $\mathrm{GHG}$ (in $\mathrm{CO}_{2 \mathrm{eq}} \mathrm{g} / \mathrm{sec}$ ) emissions were then processed based on the best GHG costing approach that was investigated in Section 5.7.1. MOVES estimated emissions by defining the vehicle operating mode, which was based on the vehicle specific power (VSP) (USEPA, 2015). Further details can be found in Section 3.4. For the myopic routing strategies, only $100 \%$ CAVs was employed in a congested traffic condition. 


\subsection{Case Study}

Unlike the existing eco-routing studies (Alfaseeh and Farooq, 2020b), this myopic multiobjective eco-routing strategies were applied in a congested urban network, during the morning peak hour, while microscopic traffic and emission models were used despite the associated complexity. The links of the case study, which is downtown Toronto, are associated with a high level of heterogeneity in terms of the speed limit, number of lanes, and the directions as demonstrated in Section 3.5.

\subsection{GHG Costing Approaches}

Before applying the myopic eco-routing strategies, different GHG costing approaches were examined, while taking into account the spatial and temporal dimensions. Table 5.1 shows the different GHG costing approaches considered following the Equations 5.1, 5.2, 5.3, 5.4 and 5.5, respectively. The best GHG costing approach was adopted for the myopic routing in this chapter and the anticipatory routing in Chapter 7 . The resolution level of the available data points was the judging factor for what costing approach to employ, while estimating the link GHG cost. When the total GHG is available at a minute level, a more spatially aggregated costing approach is used.

Table 5.1 The Investigated GHG Costing Strategies

\begin{tabular}{lc}
\hline Costing strategy name & Approach \\
\hline \hline $\mathrm{GHG}_{\text {cost } 1}$ & Sum of GHG \\
\hline $\mathrm{GHG}_{\text {cost } 2}$ & Sum of GHG per lane \\
\hline $\mathrm{GHG}_{\text {cost } 3}$ & Weighted average of GHG \\
\hline $\mathrm{GHG}_{\text {cost } 4}$ & Weighted average of GHG per lane \\
\hline $\mathrm{GHG}_{\text {cost } 5}$ & Approximate vehicle cost of GHG \\
\hline
\end{tabular}


$\mathrm{GHG}_{\operatorname{cost} 1\left(l, \Delta_{j}\right)}$, following Equation 5.1, is for when GHG cost is the sum of GHG emissions of vehicles $N$ on a studied link $l$ during and interval $\Delta_{j}$. The optimal updating interval $\Delta_{j}$ has been found to be one minute as in Section 4.6.1 (Alfaseeh, Djavadian, and Farooq, 2018). $\lambda_{(i, l, j)}$ is a binary variable, 1 if vehicle $i$ is on a studied link $l$ at time $j$ and 0 otherwise. Taking the sum of GHG may not be very representative as links in a case study may be associated with different number of lanes. Thus, $\mathrm{GHG}_{\operatorname{cost} 2\left(l, \Delta_{j}\right)}$ normalizes the total GHG cost based on number of lanes $Z_{l}$ as in Equation 5.2. To consider a higher temporal resolution, the $\mathrm{GHG}_{\operatorname{cost} 3\left(l, \Delta_{j}\right)}$ costing approach is examined. Weighted average of GHG produced on link $l$ is the outcome of Equation 5.3. At every second $j$ of any interval, a weight $j$, from 160 , is multiplied by the produced GHG by vehicles. The sum is then divided by the sum of weights. This costing approach takes the GHG cost at every second, which means it is associated with a higher temporal resolution compared to $\mathrm{GHG}_{\operatorname{cost} 1\left(l, \Delta_{j}\right)}$ and $\mathrm{GHG}_{\operatorname{cost} 2\left(l, \Delta_{j}\right)}$. $\mathrm{GHG}_{\operatorname{cost} 4\left(l, \Delta_{j}\right)}$ as in Equation 5.4 (Alfaseeh, Djavadian, Tu, et al., 2019) follows the same logic of $\mathrm{GHG}_{\operatorname{cost} 3\left(l, \Delta_{j}\right)}$, but divides by the number of lanes $Z_{l}$ of link $l$ to normalize. Finally, $\mathrm{GHG}_{\operatorname{cost5}\left(l, \Delta_{j}\right)}$ is the approximate vehicle cost of GHG traversing a studied link $l$. The approximate vehicle cost of GHG depends on an estimated GHG emission rate (ER) and TT of interval $\Delta_{j}$ on link $l$ following Equation 5.5 (Djavadian, Alfaseeh, et al., 2020; Djavadian, Tu, et al., 2020). TT was estimated following Equation 5.6, where $D_{l}$ and $V\left(l, \Delta_{j}\right)$ represent link $l$ length and space mean speed on link $l$ of time interval $\Delta_{j}$, respectively. With respect to the simulation and how the above variables of GHG and TT were estimated, MOVES has been incorporated to estimate the second-by-second GHG of every vehicle following the steps in Section 3.4. Traffic variables, such as speed and acceleration were used as inputs for MOVES. For $\mathrm{GHG}_{\operatorname{cost} 1\left(l, \Delta_{j}\right)}$ and $\mathrm{GHG}_{\operatorname{cost} 2\left(l, \Delta_{j}\right)}$ costing approaches, the sum of the second-bysecond estimated GHG from MOVES of vehicles on link $l$ during the interval $\Delta_{j}$ was used to obtain the total GHG produced during the interval $\Delta_{j}$. In contrast, for the $\mathrm{GHG}_{\operatorname{cost}\left(l, \Delta_{j}\right)}$, and $\mathrm{GHG}_{\operatorname{cost} 4\left(l, \Delta_{j}\right)}$ costing approaches, at every second the summation of GHG produced by vehicles on link $l$ was the input to the equations related. For the TT estimation, the traffic 
simulator utilized the IDM car-following model, as in Section 3.3, to estimate the vehicular speed from the acceleration at every second. This means that speed $V\left(l, \Delta_{j}\right)$ was calculated based on the second-by-second data points of speed and updated at every one minute at link level (Djavadian and Farooq, 2018).

$$
\begin{gathered}
G H G_{\operatorname{cost} 1\left(l, \Delta_{j}\right)}=\sum_{j=1}^{\Delta_{j}} \sum_{i=1}^{N} \lambda_{(i, l, j)} \times G H G_{(i, j)} \\
G H G_{\operatorname{cost} 2\left(l, \Delta_{j}\right)}=\frac{\sum_{j=1}^{\Delta_{j}}\left(\sum_{i=1}^{N} \lambda_{(i, l, j)} \times G H G_{(i, j)}\right)}{Z_{l}} \\
G H G_{\operatorname{cost} 3\left(l, \Delta_{j}\right)}=\frac{\sum_{j=1}^{\Delta_{j}\left(j \times \sum_{i=1}^{N} \lambda_{(i, l, j)} \times G H G_{(i, j)}\right)}}{\sum_{j=1}^{\Delta_{j}} j} \\
G H G_{\operatorname{cost} 4\left(l, \Delta_{j}\right)}=\frac{\sum_{j=1}^{\Delta_{j}}\left(j \times \frac{\left.\sum_{i=1}^{N} \lambda_{(i, l, j)} \times G H G_{(i, j)}\right)}{Z_{l}}\right.}{\sum_{j=1}^{\Delta_{j}} j} \\
G T_{\left(l, \Delta_{j}\right)}=\frac{D_{l}}{V\left(l, \Delta_{j}\right)} \\
G H G_{E R\left(l, \Delta_{j}\right)} * T T_{\left(l, \Delta_{j}\right)}
\end{gathered}
$$

\subsection{Routing Strategies}

Table 5.2 illustrates the examined routing strategies in this chapter. The first routing strategy optimized TT, while the second and third ones optimized GHG and TT\&GHG, respectively. Minimizing TT may have an impact on the environmental indicators, GHG and NOx. While optimizing TT, higher speed links or longer routes of higher speed may be adopted, 
which introduces more GHG and NOx emissions due to the quasi-convex relationship between speed and GHG (Djavadian, Tu, et al., 2020). On the other hand, optimizing GHG must have an effect on the traffic indicators, TT, VKT, speed, density, and flow, as GHG estimation depends mainly on speed. Illustrating the trade-offs is an essential element to be considered when the results are discussed of the different routing strategies. As recommended by Law and McComas (1991), every routing strategy has been run for five replications of different seeds and the average of any indicator was taken to account for stochasticity. The used updating interval $\left(\Delta_{j}\right)$ was one minute. This means that at every minute the cost of links was updated whether it was TT, GHG, or TT\&GHG. Referring to the general optimization formula, Equation 5.7 was followed. Where $T T_{l}$ is travel time on link $l$ and $G H G_{l}$ is the GHG emissions (in $\mathrm{CO}_{2 \mathrm{eq}}$ ) on link $l ; n$ is number of links of a path $k$; and $W_{T T}$ and $W_{G H G}$ are the weights associated with travel time and GHG emissions, respectively. It is worth mentioning that variables in Equation 5.7 (TT and $G H G$ ) have different units and weights transformed them into a consistent unit, such as monetary value, when multi-objective routing was applied. The adopted TT monetary value was $\$ 27.36 /$ hour based on Statistics Canada (CBC, 2019). The used monetary value of GHG emission was $\$ 15.77 /$ Ton based on The World Bank (2019). Before applying the different routing strategies, the different GHG costing approaches were analyzed in Section 5.7.1 and the most suitable one was used throughout this dissertation. The adopted performance indicators to compare between the myopic routing strategies were average travel time (TT), average vehicle kilometres travelled (VKT), total GHG, and total NOx produced. To further analyze the outcomes of myopic routing strategies, paths of a random vehicle were applied as presented in Section 5.7.3 in addition to the network level analysis in Section 5.7.4.

$$
\min \left\{\sum_{l=1}^{n} W_{T T} \cdot T T_{l}+\sum_{l=1}^{n} W_{G H G} \cdot G H G_{l}\right\}
$$


Table 5.2 The Considered Routing Strategies

\begin{tabular}{lc}
\hline Scenario name & Routing type \\
\hline \hline $\mathrm{TT}_{m}$ & Myopic \\
\hline $\mathrm{GHG}_{m}$ & Myopic \\
\hline $\mathrm{TT}_{\mathrm{GHHG}}$ & Myopic \\
\hline
\end{tabular}

\subsection{Results and Analysis}

First, the GHG costing approaches are analyzed in terms of the mean TT, mean VKT, total GHG, and total NOx in Section 5.7.1. Then, the myopic routing strategies are discussed in detail. The mean TT, mean VKT, total GHG, and total NOx are demonstrated in Section 5.7.2. Paths of a random vehicle of the myopic different routing strategies are extracted and findings are presented in Section 5.7.3 to better comprehend the difference between the routing strategies. The network level average speed, GHG, and NOx over time are examined in Section 5.7.4 to illustrate the impact of adopting different routing objectives at the network level.

\subsubsection{GHG Costing Approach Analysis}

Figure 5.2 shows that the $\mathrm{GHG}_{\text {cost } 1}$ strategy performed the worst, in terms of all the performance indicators. This is due to the fact that traffic characteristics were not captured of a studied links, such as speed, flow, or density. The same total GHG emission cost can be for two dramatically different sets of link characteristics. For instance a high total emission $x$ can be for a lightly used very long link of four lanes or for a shorter congested link of two lanes. The speed, flow, and density of the aforementioned two cases are dramatically different. That is, taking the total emissions did not reflect on spatial and temporal changes of both traffic and environmental indicators. 


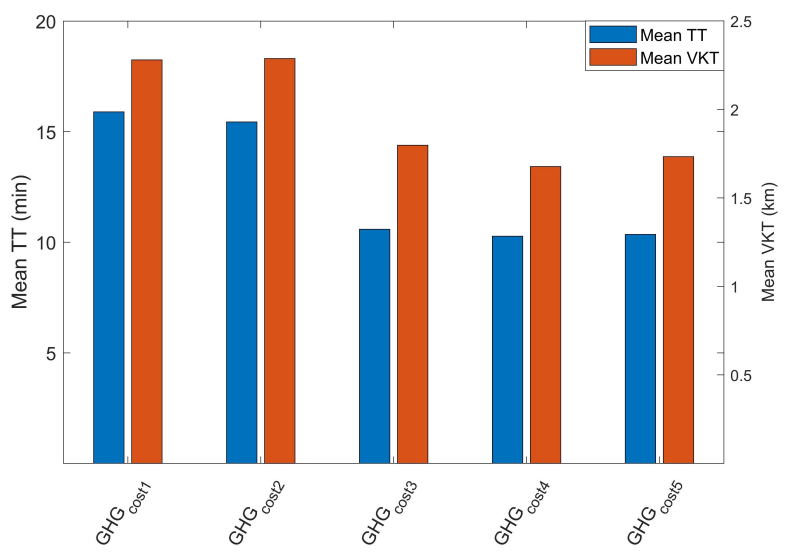

(a)

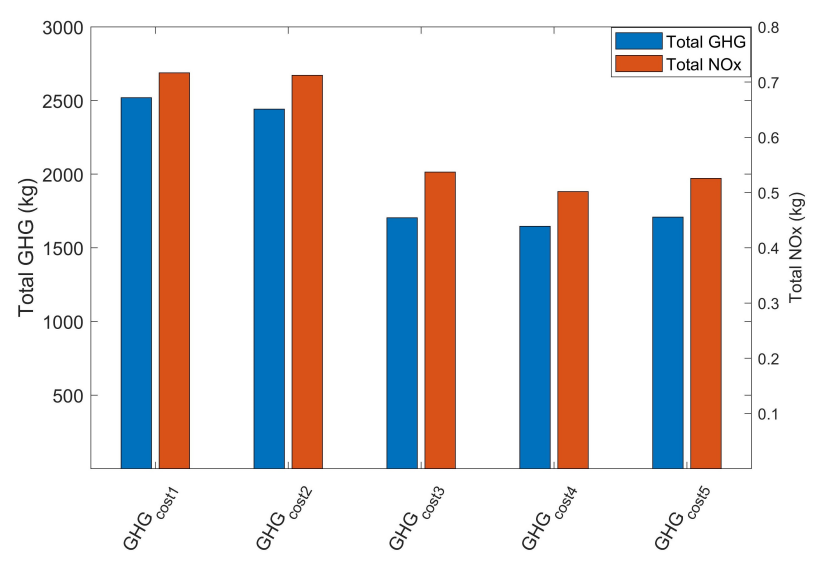

(b)

Figure 5.2 The Impact of Different GHG Costing Approaches on the a) Mean TT and VKT and b) Total GHG and NOx

The $\mathrm{GHG}_{\text {cost3 }}$ costing approach illustrated significant improvements compared to the $\mathrm{GHG}_{\text {cost1 }}$ approach. The former is associated with a reduction in average TT, average VKT, total GHG, and total NOx of 33\%, 21\%, 32\%, and 25\%, respectively, compared to the latter. The main justification is the high level of temporal resolution employed. The changes in the environmental status on links are sensitive to the temporal dimension. In general, if the link cost is updated in a timely manner, vehicles would be routed to links representing the environmental status reliably. Giving higher weights to the most recent seconds contributed to the enhancement in terms of the four performance indicators. In other words, GHG emissions of second 1 of interval $\Delta_{j}$ were multiplied by 1 , while emissions in second 60 of interval $\Delta_{j}$ were multiplied by 60 . If produced GHG emissions at second 1 and 60 were equal, the contribution of second 60 to link GHG cost was significantly higher due to the higher weight associated, as it reflected on most recent environmental condition of a studies link. This approach required produced emissions by vehicles at every second, which is not always feasible. Obtaining the second-by-second emissions of every vehicle requires high level of computational power and memory when the dynamics in the network are simulated. Even when real data are used, a large number of sensors are required to be distributed to capture 
the second-by-second emissions produced by vehicles.

Dividing by the number of lanes in both $\mathrm{GHG}_{\text {cost2 }}$ and $\mathrm{GHG}_{\operatorname{cost} 4}$ is associated with slight enhancements compared to $\mathrm{GHG}_{\operatorname{cost} 1}$ and $\mathrm{GHG}_{\text {cost } 3}$, respectively. When two links have the same total GHG or same weighted average, but they are associated with different number of lanes, comparing their GHG cost requires normalizing based on number of lanes. In terms of mean TT, both $\mathrm{GHG}_{\text {cost } 2}$ and $\mathrm{GHG}_{\text {cost } 4}$ are associated with the same reduction of almost $3 \%$ compared to $\mathrm{GHG}_{\text {cost } 1}$ and $\mathrm{GHG}_{\text {cost } 3}$, respectively. Similarly, VKT, GHG, and NOx have been decreased by comparable magnitude to the mean TT in the case of $\mathrm{GHG}_{\text {cost } 2}$

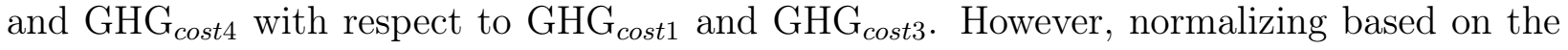
number of lanes triggers underestimation for links with larger number of lanes, which makes it unrealistic costing approach. This is due to the fact that traffic indicators, such as density and/or flow are not reliably represented. After developing video animation of every routing strategy, it was found that links of larger number of lanes were more deployed when GHG cost was normalized by the number of lanes.

$\mathrm{GHG}_{\text {cost } 5}$, which is the approximate vehicle cost of GHG traversing link $l$, is based on GHG ER and TT of a studied link $l$. The results are very much comparable to $\mathrm{GHG}_{\text {cost }}$ despite the significant difference in the employed temporal resolution. $\mathrm{GHG}_{\text {cost } 4}$ costing approach required the second-by-second produced GHG emissions by vehicles of a studied link, but did not not include any of the link traffic indicators (speed, flow, density). On the other hand, $\mathrm{GHG}_{\text {cost } 5}$, which takes into account speed and distance of a studied link, adopted the same spatial level of resolution, link level, compared to $\mathrm{GHG}_{\text {cost } 4}$, but lower temporal level of resolution, one minute, compared to $\mathrm{GHG}_{\operatorname{cost} 4}$. The high level of temporal resolution of $\mathrm{GHG}_{\text {cost } 4}$ made the results comparable to $\mathrm{GHG}_{\text {cost } 5}$. $\mathrm{GHG}_{\text {cost } 5}$ performed better than $\mathrm{GHG}_{\text {cost } 4}$ because it considered speed and distance travelled, despite the lower temporal resolution with respect to $\mathrm{GHG}_{\text {cost } 4}$. $\mathrm{GHG}_{\text {cost } 5}$ surpassed $\mathrm{GHG}_{\text {cost } 4}$ in terms of the mean TT, mean VKT, total GHG, and total NOx by $1 \%, 3 \%, 4 \%$, and $5 \%$, respectively. $\mathrm{GHG}_{\text {cost } 5}$ is the most representative and suitable costing approach as it depends not only on GHG 
ER (explicitly), but also on travel time (implicitly), speed, and distance of a studied link. This was applied while utilizing link level (spatial resolution) and one minute (temporal resolution).

With respect to the total produced GHG and NOx, it was noticed that they were dependent on the amount of time vehicles spend in the network. In other words, the higher the average TT, the higher the GHG and NOx as in Figure 5.2b. Among the investigated GHG costing approaches, $\mathrm{GHG}_{\text {cost } 5}$ is the only costing approach that reflected on traffic condition by considering speed and distance travelled. Hence, $\mathrm{GHG}_{\cos t 5}$ was the cost used for the myopic and anticipatory routing strategies in this Chapter and Chapter 7, respectively.

\subsubsection{Average TT, Average VKT, Total GHG, and Total NOx Analysis of the Different Myopic Routing Strategies}

Figure 5.3 illustrates that $\mathrm{GHG}_{m}$ routing strategy outperformed the $\mathrm{TT}_{m}$ in terms of all the four performance indicators. When TT was the routing objective, all of possible routes were investigated, while minimizing TT only. Vehicles were distributed in the network to avoid congested links. This means that vehicles were directed to uncongested links that could be of low speed and shorter length or of long distance and higher speed. This justifies the increase in mean $\mathrm{TT}$ and VKT in the case of $\mathrm{TT}_{m}$ compared to $\mathrm{GHG}_{m}$. In the case of $\mathrm{TT}_{m}$, longer routes were considered as long as they satisfy the optimization objective. On the other hand for $\mathrm{GHG}_{m}$, GHG ER and spent TT on possible routes were optimized. In other words, links of the optimal path must be the ones of the least GHG expected to be produced by one vehicle traversing them, which is dependant on GHG ER, speed, and distance. For instance, if two links have the same GHG ER, but different expected travel time, the one of less TT is chosen to satisfy the optimization objective. This means that

$\mathrm{GHG}_{m}$ looks for the best combination between GHG ER, speed, and distance to satisfy the optimization objective. $\mathrm{GHG}_{m}$ contributed to reductions in average TT, average VKT, total 
GHG, and total NOx of $15 \%, 12 \%, 11 \%$, and $13 \%$, respectively, compared to $\mathrm{TT}_{m}$. It was noticed that the reduction in TT was larger than the reduction in the total GHG. In the approximate vehicle cost of GHG as in Equation 5.5, travel time is a major component of the optimization process. While minimizing GHG cost, time spent was minimized indirectly. The aim of the approximate vehicle cost of GHG is to look for the best combination of GHG ER and TT. This demonstrates the importance of reducing TT to minimize GHG produced. Furthermore, while TT was minimized, NOx was minimized too. When vehicles spent less time in the network, less NOx was the outcome.

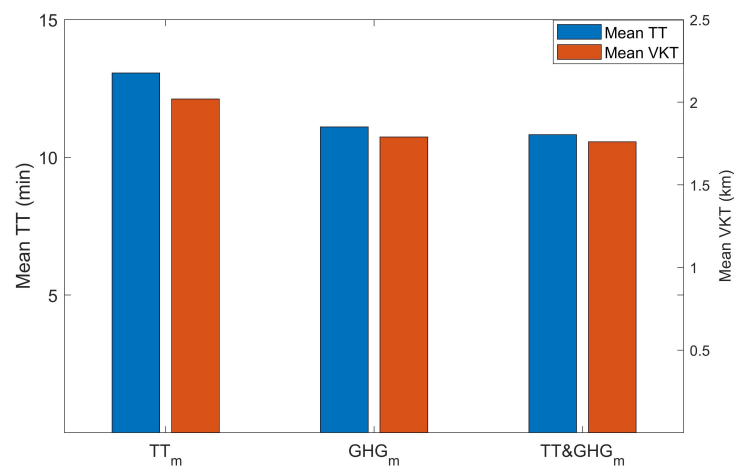

(a)

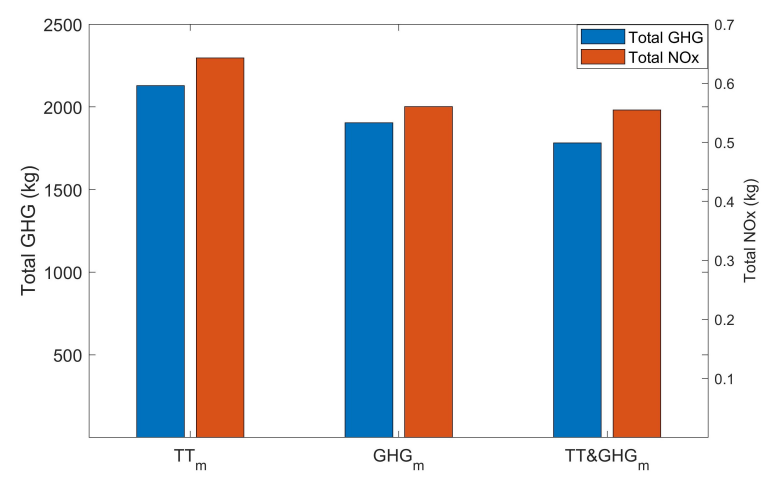

(b)

Figure 5.3 The Impact of the Different Myopic Routing Strategies on the a) Mean TT and VKT and b) Total GHG and NOx

Multi-objective, TT\& $\mathrm{GHG}_{m}$, routing strategy triggered a slight improvement compared to $\mathrm{GHG}_{m}$. The former contributed to reductions in the average TT, average VKT, total GHG, and total NOx of $2 \%, 1 \%, 6 \%$, and $1 \%$, respectively, compared to the latter. This is stemmed from the fact that GHG cost has been scaled to be comparable to TT cost of studied links. It was noticed that there was an obvious difference between the scale of TT and approximate vehicle cost of GHG of links. To make the cost of the two variables of the same scale, TT and GHG costs of the most congested link over time have been compared to define the optimal scaling factor. When TT and GHG were optimized simultaneously, the GHG cost was multiplied by a factor of $10^{5}$, where GHG cost on links was taken by 
tons and time unit of minute in the optimization formula. In the case of TT\&GHG$G_{m}$, not only TT was optimized, but also GHG. In the case of multi-objective routing, both TT and GHG objectives aimed at reducing the TT spent in the network. While TT objective minimized TT explicitly, GHG objective minimized TT implicitly. Combining both TT and GHG means controlling TT spent more strictly compared to routing to minimize TT or GHG solely. It is noticed that there is a proportional relationship between experienced TT \& VKT and produced GHG \& NOx on links. In other words, the more TT and longer trips vehicles experience in the network, the more produced GHG and NOx as illustrated in Figure 5.3. GHG estimation in Equation 5.5 is a function of time. This explains the increase in GHG when TT increases and similarly for NOx. To sum it up, when TT was the routing objective all what mattered was TT spent regardless of VKT, GHG, or NOx, while when GHG was part of the optimization process, GHG and TT were minimized while VKT is controlled.

\subsubsection{Path Analysis of the Different Myopic Routing Strategies}

This analysis was conducted at a single vehicle level. Figure 5.4 illustrates the paths of a randomly chosen vehicle of the different routing strategies $\left(\mathrm{TT}_{m}, \mathrm{GHG}_{m}\right.$, and TT\&GHG $\left.\mathrm{G}_{m}\right)$. From the figure, re-routing is a dominating feature in the three routing strategies. This could be due to the fact that link cost was captured at link level and at every one minute. At minute $(t)$, the link cost was estimated based on the previous minute $(t-1)$. The traffic and environmental conditions at minute $(t)$ changed, which was not reflected on link cost, which would remain based on the data of the previous minute $(t-1)$. The re-routing phenomenon implies that increasing the spatial and temporal level resolution while estimating link cost may contribute to further improvements. In addition, estimating more proactively link cost, by capturing traffic and environmental evolution, may introduce a better outcome, which is discussed in detail in Chapter 7.

For $\mathrm{TT}_{m}$, the route was longer compared to other routing strategies, which supports the 
finding of the previous section related to mean TT and mean VKT. For $\mathrm{TT}_{m}$, all of the possible paths were examined and vehicles were routed to the ones of the least TT regardless of experienced VKT, GHG, and NOx. It can also be noticed that there are mutual links between $\mathrm{GHG}_{m}$ and $\mathrm{TT} \& \mathrm{GHG}_{m}$ as $\mathrm{GHG}$ was part of the optimization process in both routing strategies. Finally, the path of $\mathrm{TT} \& \mathrm{GHG}_{m}$ is the shortest compared to the other two strategies. The length of paths of $\mathrm{TT}_{m}, \mathrm{GHG}_{m}$, and $\mathrm{TT \& GHG} \mathrm{G}_{m}$ was 3.7, 3.2, and $3.1 \mathrm{~km}$, respectively. When GHG was part of the optimization objective, the approximate vehicle cost of $\mathrm{GHG}$ of $\mathrm{GHG}_{m}$ considered not only produced GHG of one vehicle, but also speed and VKT indirectly. Links of optimal speed were prioritized as long as the objective was minimized. Links of too high or too low speed were not in favour due to the adverse impact on the GHG ER and consequently GHG final cost.

\subsubsection{Network Level Analysis of the Different Myopic Routing Strategies}

This analysis was conducted at the network level to comprehend the impact of the proposed routing strategies at a higher level. Figure 5.5a shows the average speed over time in the network of the different myopic routing strategies. In the case of $\mathrm{GHG}_{m}$ and $\mathrm{TT} \& \mathrm{GHG}_{m}$, the network was loaded and unloaded quicker, in which it took $8 \%$ and $11 \%$ less time, respectively, compared to $\mathrm{TT}_{m}$. Mean $\mathrm{TT}$ and mean VKT analysis in addition to the path analysis is Sections 5.7.2 and 5.7.3, respectively, support the longer required time to empty the network in the case of $\mathrm{TT}_{m}$ compared to the other two myopic routing strategies, $\mathrm{GHG}_{m}$ and $\mathrm{TT} \& \mathrm{GHG}_{m}$. It is noticed that $\mathrm{GHG}_{m}$ and $\mathrm{TT} \& \mathrm{GHG}_{m}$ were associated with higher average speed over time compared to $\mathrm{TT}_{m}$. As much as 12 and $25 \mathrm{~km} /$ hour increase in speed was observed at certain points of time for $\mathrm{GHG}_{m}$ and TT\&GHG${ }_{m}$, respectively, compared to $\mathrm{TT}_{m}$. 


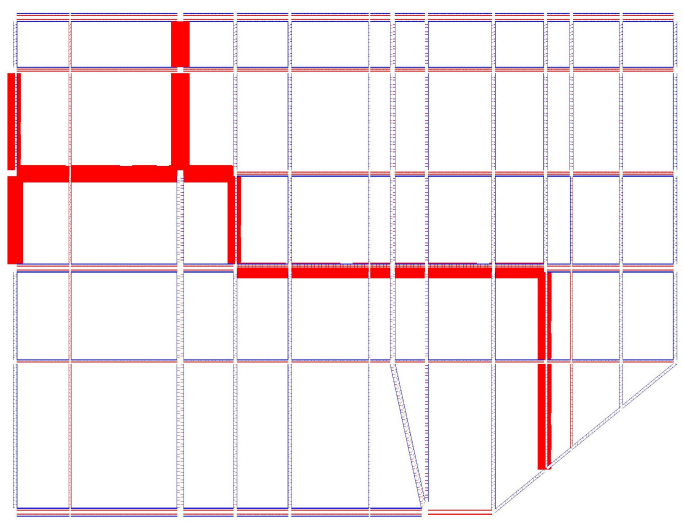

(a)

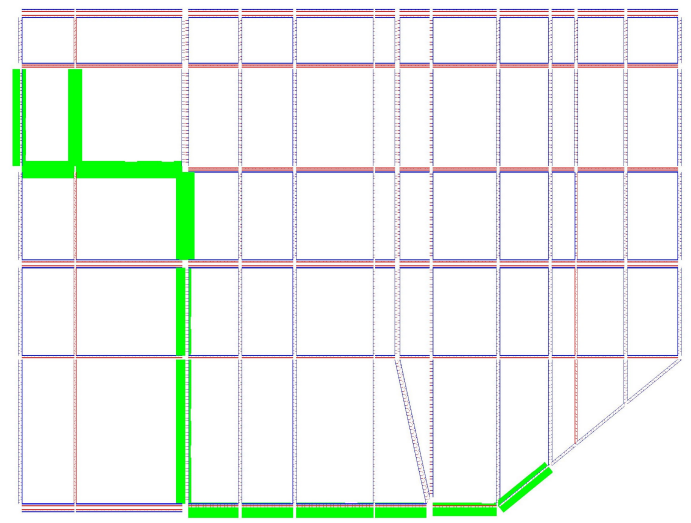

(b)

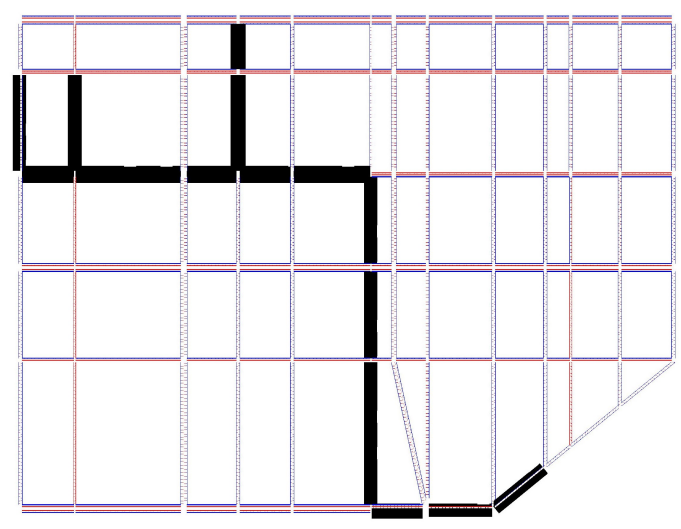

(c)

Figure 5.4 Myopic Routes of a Random Vehicle of the Different Routing Strategies, a) TT, b) GHG, and c) TT\&GHG

When TT was the routing objective, all of available paths were checked and the best one was chosen based only on one criterion, TT, regardless of experienced speed, distance, GHG, and NOx. While optimizing TT, vehicles might be sent to links of lower speed and shorter distance or to routes of higher speed and longer distance to minimize TT cost. Nevertheless, when GHG was part of the optimization objective, GHG ER, speed, and distance were parts of cost estimation process of links as shows in Equations 5.5 and 5.6. The best combination of the aforementioned variables was chosen that satisfied the optimization objective. Figures 5.3a and 5.4 support the aforementioned justification as when GHG was part of the optimization process, mean TT and mean VKT and re-routing was less compared 


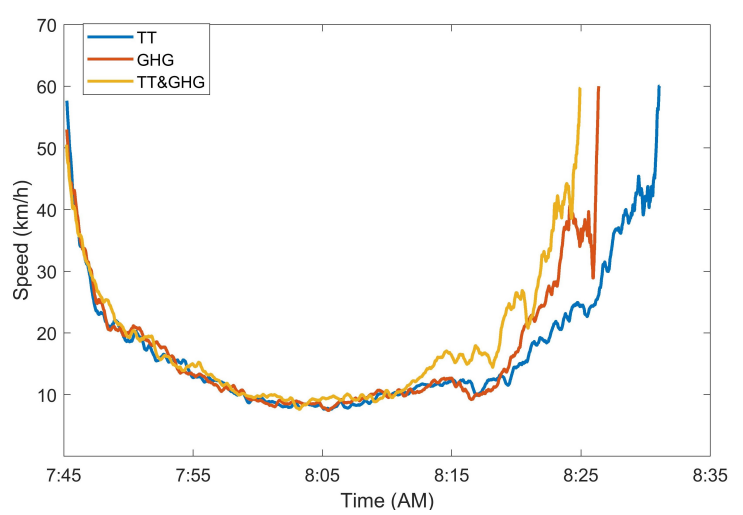

(a)

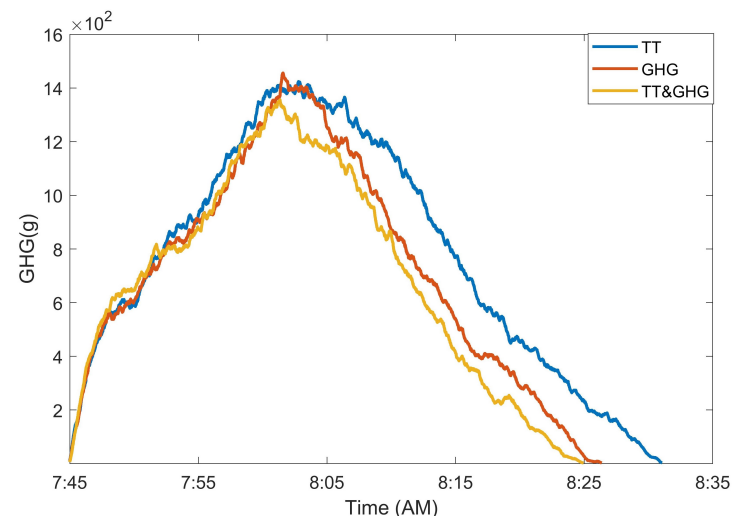

(b)

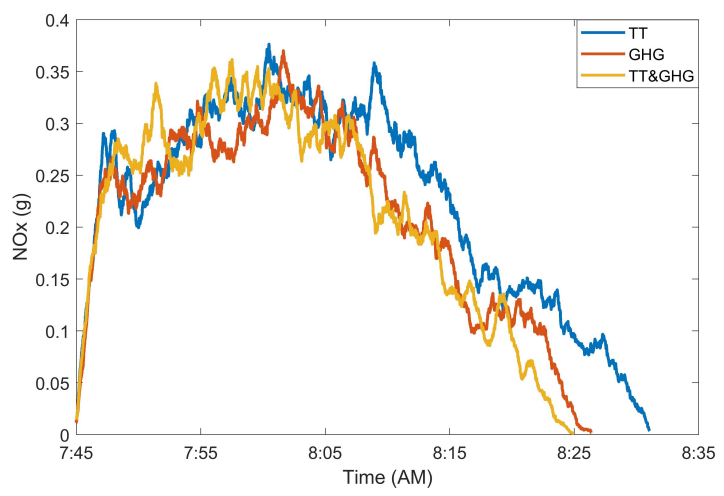

(c)

Figure 5.5 The Network under the Different Myopic Routing Strategies and its a) Speed, b) GHG, and c) NOx over time

to when TT was the only objective. When GHG was minimized, too high or too low speed was not in favour as either case contributes to higher cost. Average TT and average VKT were smaller in the case of $\mathrm{GHG}_{m}$ and $\mathrm{TT} \& \mathrm{GHG}_{m}$, this means that on average higher speed links were employed while routing vehicles to their destinations compared to $\mathrm{TT}_{m}$. It is important to note that the peak period of the simulation was between 8:00am and 8:20am. From 7:45am, when the demand started being loaded till around 8:10am average speed of the three myopic routing strategies was comparable. However, after 8:10am, variation in speed of the different routing objective strategies was obvious. This demonstrates the impact of emptying the network faster, which was triggered by reduced TT and VKT. After 8:10am, $\mathrm{GHG}_{m}$ and $\mathrm{TT \& GHG}$ were associated with higher speed over time, as less vehicles were 
in the network and links were chosen based on their GHG ER, speed, and length, compared to $\mathrm{TT}_{m}$.

Figures 5.5b and 5.5c illustrate that $\mathrm{GHG}_{m}$ and TT\& $\mathrm{GHG}_{m}$ are associated with less GHG and NOx over time compared to the $\mathrm{TT}_{m}$. The reduction in GHG over time is as much as 0.25 and $0.4 \mathrm{~kg}$ at certain points for $\mathrm{GHG}_{m}$ and $\mathrm{TT} \& \mathrm{GHG}_{m}$, respectively, compared to $\mathrm{TT}_{m}$. The explanation is the difference between the costing approaches of $\mathrm{TT}_{m}$ and $\mathrm{GHG}_{m}$. The former minimizes only TT, while the latter considers GHG ER and other traffic indicators to minimize the final cost. For NOx, the value is high from the beginning of the simulation, which is due to high speed of vehicles entering the network. It is also noticed that there is a lot of fluctuations caused by sensitivity to speed on links and also number of vehicles in the network. This assures that spending less time in the network and travelling for shorter distances have a desirable impact on the environmental dimension. The difference in total GHG between the different routing strategies after 8:00am is more noticeable. This is due to less number of vehicles in the network for $\mathrm{GHG}_{m}$ and $\mathrm{TT} \& \mathrm{GHG}_{m}$ compared to $\mathrm{TT}_{m}$.

\subsection{Concluding Remarks}

In this chapter, myopic single- and multi-objective routing strategies were examined, while employing the E2ECAV dynamic distributed routing framework introduced in Chapter 4. The major findings of this chapter are as follows. When TT was the only routing objective, travel time was all what mattered. All of possible routes in the network were examined, regardless of experienced speed, VKT, GHG, and NOx. This illustrates why routing based on TT was the worst routing strategy in terms of the whole performance indicators. In addition, when TT was the routing objective, longer routes of higher speed were chosen, which contributed to more GHG and NOx emissions. When GHG was the routing objective, the approximate vehicle cost of GHG was the best costing approach due to its ability to take into account GHG ER of every link in addition to travel time. Routing based on GHG 
contributed to significant enhancements compared to routing based on TT, due to the costing approach reflecting on both traffic and environmental dimensions at once. The approximate vehicle cost of GHG took into account not only GHG ER but also the experienced speed and distance on links in concern. That is, the best combination of the aforementioned three variables was chosen. Routing based on TT\&GHG introduced further enhancements compared to when GHG was the routing objective. Due to different scales of TT and GHG variables, a scaling factor was used, for GHG cost, to give the same importance for the two optimized variables, TT and GHG, contributing to improvements in the case of multiobjective routing. In the case of TT\&GHG routing strategy, travel time was minimized more strictly as TT routing objective optimized it explicitly, while GHG routing objective minimized it implicitly. Comparing TT to TT\&GHG routing strategies is like comparing User Equilibrium (UE) to System Optimal (SO) traffic assignment. The network was loaded and unloaded quicker in the case of when GHG and when TT\&GHG were the routing objectives compared to when TT was the only minimized variable. When GHG was part of the optimization process, the time spent in the network was reduced implicitly triggering improvements at the network and vehicle level. The amount of produced NOx is very sensitive to aggressive driving. When GHG was the routing objective, links of speed close to optimal value were prioritized and mean TT and VKT were reduced. These factors affected the produced NOx implicitly. Due to re-routing observed in the three routing strategies, there is still room for improvements. However, this requires predicting traffic and environmental conditions for a proactive routing. In Chapter 6, deep learning based on the Long Short-Term Memory (LSTM) predictive models were developed for speed and GHG ER. The developed predictive models were deployed for the anticipatory routing application in Chapter 7 for further enhancements from traffic and environmental perspectives. 


\section{Chapter Six}

\section{Prediction Models}

This chapter has resulted in the following publication:

1. Alfaseeh, Lama, Tu, Ran, Farooq, Bilal, \& Hatzopoulou, Marianne (2020). "Greenhouse Gas Emission Prediction on Road Network Using Deep Sequence Learning". In: Transportation Research Part D: Transport and Environment. 


\subsection{Introduction}

Topics related to GHG reduction have grabbed the attention of researchers in the transportation field. In the near future, adoption of smart cities technologies will make high resolution traffic related information available to improve several applications, such as prediction and routing. It has been proposed that anticipatory routing can bring in further improvements compared to myopic routing (Ben-Akiva et al., 2001; Bottom, 2000; Mahmassani, 1994). The conventional routing used nowadays is myopic (Elbery and Rakha, 2019; Bandeira et al., 2018; Ahn and Rakha, 2013), which depends on previous time step information and does not consider the evolution of the traffic and environmental conditions. On the other hand, anticipatory routing is based on predicted values of traffic and/or environmental variables of current time step (Bottom, 2000). In other words, links cost in case of myopic routing is taken at time $(t-1)$, while link cost in case of anticipatory routing is at time $(t)$. Hence, for real-time anticipatory applications, efficient predictive models are required, which consider a proper level of spatial and temporal resolution.

Based on the findings in Chapter 5, with respect to the best GHG costing approach, predictive models of GHG ER and speed were developed in this chapter to consider TT, GHG, TT\&GHG routing objectives for the anticipatory multi-obejctive eco-routing application in Chapter 7. It is worth mentioning that some pollutants, such as Methane (CH4) and Nitrous oxide (N2O), other than $\mathrm{CO}_{2}$ contribute to climate change. The aforementioned pollutants may have an impact that is greater than that of $\mathrm{CO}_{2}$ and is often referred to as " $\mathrm{CO}_{2}$ equivalent" (United States Environmental Protection Agency, 2020). This explains why $\mathrm{CO}_{2} \mathrm{ER}$ was predicted in this chapter in the form of $\mathrm{CO}_{2 \text { eq }}$ instead of $\mathrm{CO}_{2}$. Speed was predicted instead of TT due to the direct relationship between speed and traffic predictors, such as density, and flow. Three predictive models were examined, ARIMA, clustering, and LSTM for GHG ER prediction. A comparison between the three proposed predictive models was applied, in terms of spatial and temporal aspects, and the best model was considered 
for a detailed analysis. Matlab was the used programming platform to develop and analyze the aforementioned three prediction models. The best predictive model was then deployed to forecast speed. Predictive models require data points in general. Some models, such as clustering and LSTM need a lot of representative data points. The performance quality of a predictive model is a function of data points quality. Due to scarcity of real data to develop predictive models, simulation was the tool employed in this dissertation. While routing vehicles to their destinations, in the efficient E2ECAV (Farooq and Djavadian, 2019) distributed routing framework, data points were collected and processed to be in the form

required by every examined predictive model in this chapter. Nevertheless, in the near future, deployment of smart cities technologies will make real data available for prediction applications. Chapter 3 includes the details related to the traffic and emission simulators and how they intact with each other in the E2ECAV distributed routing framework.

Figure 6.1 demonstrates the framework of this chapter and its relationship with other chapters in this dissertation. This chapter is organized as follows. A brief literature review related to adopted predictive models for GHG emissions and speed is presented in Section 6.2. The methodology related to ARIMA, clustering, and LSTM is presented in Sections 6.3, 6.4, and 6.5, respectively. The data collection process is illustrated in Section 6.6 followed by the discussion in Section 6.7 and the concluding remarks in Section 6.8. The best predictive models developed in this chapter were adopted for the anticipatory routing application in Chapter 7 .

\subsection{Literature}

With reference to the used predictive models for GHG, a wide variety was found in literature, such as Grey model (A. Ö. Dengiz, Atalay, and O. Dengiz, 2018), autoregressive integrated moving average (ARIMA) (Rahman and Hasan, 2017), artificial neural networks (ANNs) (Abdullah and Pauzi, 2015), Long Short-Term Memory (LSTM) network (Ameyaw, L. Yao, 


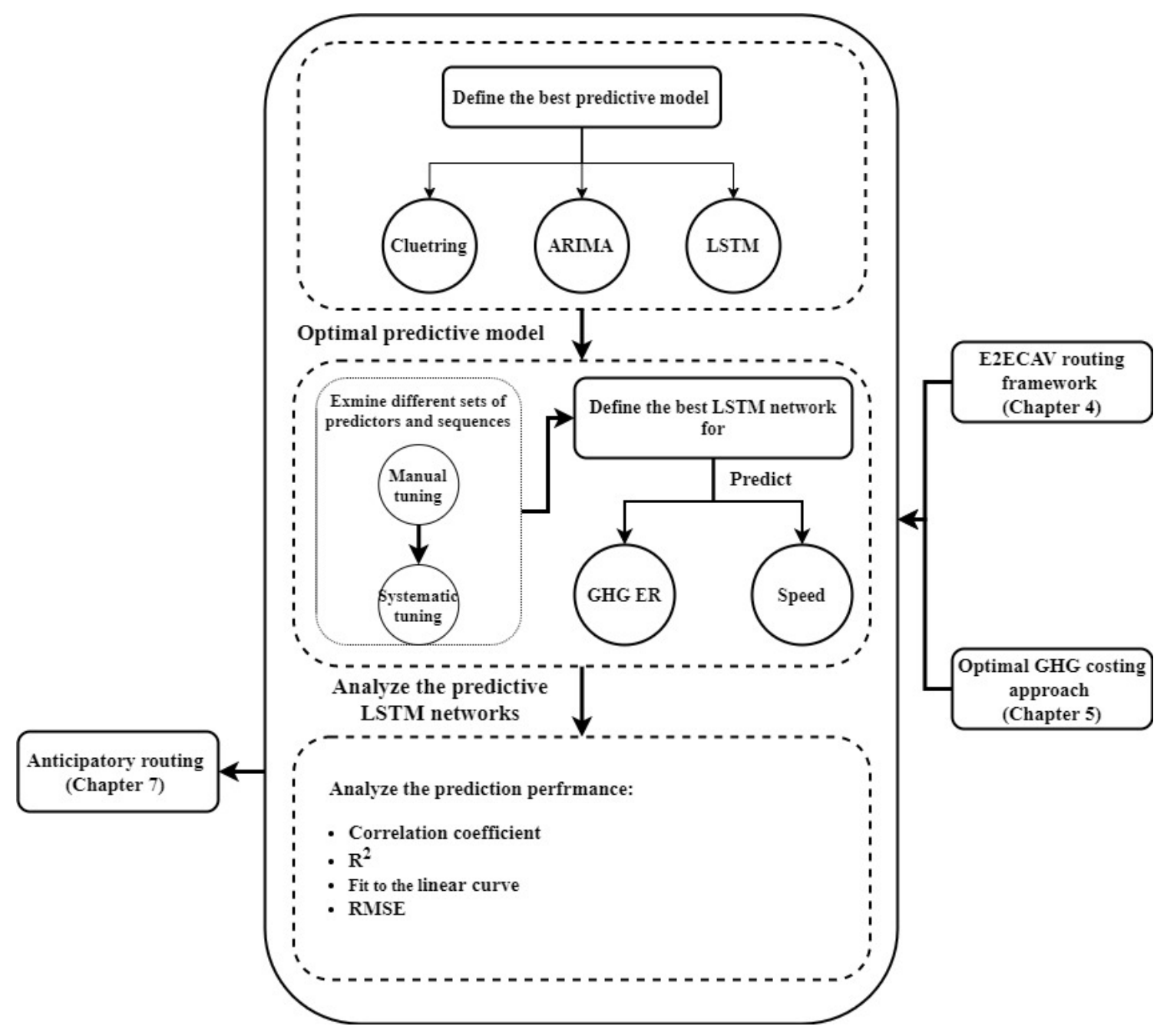

Figure 6.1 Chapter Six Framework

et al., 2019; Y. Huang, Shen, and H. Liu, 2019; Ameyaw and L. Yao, 2018), and clustering (Poucin, Farooq, and Patterson, 2018).

GHG emission has been predicted dominantly at national level regardless of the deployed models. Pao and Tsai (2011) forecasted $\mathrm{CO}_{2}$ emissions based on income and energy consumption by using grey prediction model (GM) in Brazil. Pao and Tsai (2011) found that results were comparable to ARIMA in terms of forecasting performance. The GM was also used by Lin, Liou, and C.-P. Huang (2011) to predict $\mathrm{CO}_{2}$ emissions in Taiwan. ARIMA, which is a commonly used statistical approach used in case of time series prediction, was adopted 
to predict $\mathrm{CO}_{2}$ emissions in Bangladesh and Bahrain as in Rahman and Hasan (2017) and Tudor (2016), respectively.

Antanasijević et al. (2014) predicted GHG emissions at national level as well, of European countries, employing a new approach based on ANNs. Predictors considered were agriculture, transportation, energy supply and use, and waste. Radojević et al. (2013) employed ANN to predict $\mathrm{CO}_{2}$ emissions in Serbia based on the share of renewable sources of energy, gross domestic product, gross energy consumption, and energy intensity (Radojević et al., 2013). The extreme learning machine (ELM), which is a type of ANNs, based on particle swarm (PSO) optimization was used by W. Sun, C. Wang, and C. Zhang (2017) to predict $\mathrm{CO}_{2}$ emissions. W. Sun, C. Wang, and C. Zhang (2017) found that their proposed approach, called PSO-ELM, outperformed ELM and back propagation neural network in terms of prediction performance, RMSE and MAPE. At a more disaggregated level, Grote et al. (2018) developed Practical Emissions Model for Local Authorities emission (PEMLA) to be used by local government authorities. Data points were collected from sensors, which were part of the traffic control system. Five traffic variables were taken into consideration: 1) traffic average speed (km/hour); 2) traffic density (vehicles/km); 3) traffic average delay rate (seconds/vehicle.km); 4) access density (intersections $/ \mathrm{km}$ ); and 5) square of traffic average speed $(\mathrm{km} / \text { hour })^{2}$. The relationship between variables was defined by the Multiple Linear Regression (MLR), while the ANN was used to predict the $\mathrm{CO}_{2}$. Their case study was Southampton, a city on the South coast of the UK with a population of approximately 255,000. Their prediction interval was of five minutes. Traffic variables were obtained from detectors at every five minutes to estimate network level $\mathrm{CO}_{2}$ emissions (Grote et al., 2018). The main feature of the above GHG predictive models is that models adopted an extremely low level of spatial and temporal resolution. GHG emissions were mainly predicted at a national level as in Y. Huang, Shen, and H. Liu (2019); Ameyaw, L. Yao, et al. (2019) and Ameyaw and L. Yao (2018). Even when spatial resolution was increased, temporal dimension was not captured at a disaggregated level as in Grote et al. (2018), in which five minute 
updating interval was employed. In terms of predictors used, generally fuel and economical factors were employed (Alfaseeh, Tu, et al., 2020).

Vlahogianni, Karlaftis, and Golias (2014) developed a detailed review related to shortterm traffic forecasting. It was shown that studies mainly considered freeways as their case study, due to simplicity compared to urban congested networks. The authors also found that mostly statistical models were used for prediction (Vlahogianni, Karlaftis, and Golias, 2014). With reference to TT prediction, two streams have been found in literature, one predicted TT directly and the other one predicted speed and consequently TT. For directly forecasting travel time, several predictive models have been deployed. Linear modelling (X. Zhang and Rice, 2003), the nonlinear autoregressive with external inputs (NARX) model (Mane and Pulugurtha, 2018), the nonlinear autoregressive model (NAR) (Mane and Pulugurtha, 2018), clustering (Elhenawy, H. Chen, and Rakha, 2014), neural networks (Mane and Pulugurtha, 2018), ant colony based approach (Claes and Holvoet, 2011), and deep neural networks (Duan, Lv, and F.-Y. Wang, 2016; Ran et al., 2019). For indirectly predicting travel time from speed, examples can be found in Ishak and Alecsandru (2004); Gu et al. (2019); Innamaa (2000); Yildirimoglu and Geroliminis (2013); Ma et al. (2015); B. Yao et al. (2017) and Gmira et al. (2017). Most of the aforementioned studies adopted freeways as their case study and a low level of temporal resolution. These findings are aligned with the outcome of a review paper conducted by Vlahogianni, Karlaftis, and Golias (2014) related to short-terms traffic forecasting.

Unlike the existing related work, in this chapter GHG ER and speed were predicted based on data points extracted from a congested urban network. Available traffic and environmental predictors, such as speed, density, flow, and GHG ER were employed to predict responses at a high level of spatial (link level) and temporal (every one minute) resolution. Three predictive models were investigated and the best one was chosen based on a comparison process. 


\subsection{ARIMA with Exogenous Variables}

ARIMA (p,d,q) is a statistical approach, which has been widely employed for time series data that can be made to be "stationary" by differencing of "d" order. There should be no trends in the data in order to predict using the ARIMA model efficiently. Where: "p" and "q" are small integers reflecting on the "autoregressive" (AR) and "moving-average" (MA), respectively. For a generic form of ARIMA while $r$ exogenous variables are included, Equation 6.1 is followed.

$$
\hat{y}_{t}=\mu+\sum_{i=1}^{p} \phi_{i} y_{t-i}+\sum_{k=1}^{r} \beta_{k} x_{t k}+e_{t}+\sum_{j=1}^{q} \theta_{j} e_{t-j}
$$

Where $\hat{y}_{t}$ is the response at time $t, \mu$ is the constant, $\phi_{i}$ is the AR coefficient at lag $i$, $y_{t-i}$ is the value of the variable in concern for prediction at time $t-i, \beta_{k}$ is a coefficient of exogenous variable $k, x_{t k}$ is exogenous variable $k$ at time $t, \theta_{j}$ is the MA coefficient at lag $j$, and $e_{t-j}=y_{t-j}-\hat{y}_{t-j}$ is the forecast error that was made at period $t-j$ (Box et al., 2015).

Figure 6.2a presents the stages followed to develop ARIMA model and predict GHG ER. A ratio of $80 \%$ to $20 \%$, training to validation, was considered. To define the optimal parameters $(\mathrm{p}, \mathrm{d}, \mathrm{q})$, an iterative process took place while considering auto-correlation, partial auto-correlation plots of differenced series, and root unit which was a measure that signalized when time series was under or overdifferenced (Alfaseeh, Tu, et al., 2020). To avoid overfitting, we examined several sets of data and made sure there were no trends in the data set. One of the major shortcomings of ARIMA models in our context is related to scaleability aspect. A separate model has to be developed for every link. In other words, ARIMA lacks the spatial dimension. 


\subsection{Clustering}

Clustering is a machine learning approach, which basically groups objects so that in one group objects are more related to each other than those in other groups (Mann and Kaur, 2013). To classify traffic conditions at link level, K-means clustering has been deployed. The data points were classified into clusters and every cluster had its own centroids based on considered variables. Every data point belongs to a cluster with the minimum distance to related centroid/s of that cluster. In K-means clustering, optimal cluster is defined when total intra-cluster variance or squared error is minimized (Poucin, Farooq, and Patterson, 2018) following Equation 6.2. Taking the overfitting aspect into account, cross validation has been applied for 100 iterations during the training process of the model. For every cross-validation iteration, the training data of $80 \%$ was divided randomly into $70 \%$ and $10 \%$. The optimal GHG ER for every cluster of the $70 \%$ data points was specified based on the least distance between true and estimated GHG ER. Then the optimal GHG ER was adopted for the cross validation on the $10 \%$ data points.

$$
M=\sum_{m=1}^{k} \sum_{i=1}^{n}\left\|x_{i}^{(m)}-c_{m}\right\|^{2}
$$

Where $M$ is the objective function, $k$ is the number of clusters, $n$ is the number of data

points (observations), $x_{i}^{(m)}$ is the observation $i$ being tested for cluster $m, c_{m}$ is the centroid of cluster $m$. The number of clusters is dependent on the data and a statistical analysis associated. To specify the optimal number of clusters, the elbow method/sum of squared error (Poucin, Farooq, and Patterson, 2018), which estimates the sum of squared distances between the points within a cluster was the guide (Alfaseeh, Tu, et al., 2020). Figure 6.2b shows the steps followed. For clustering, dataset has been divided into $80 \%$ and $20 \%$ for training and validation, respectively ( $\mathrm{Tu}$, Kamel, et al., 2018). The input variables were used to define the optimal cluster they belong to based on the sum of squared distance with the centroid related. Then the response variable was obtained from the optimal cluster 
(Alfaseeh, Tu, et al., 2020).

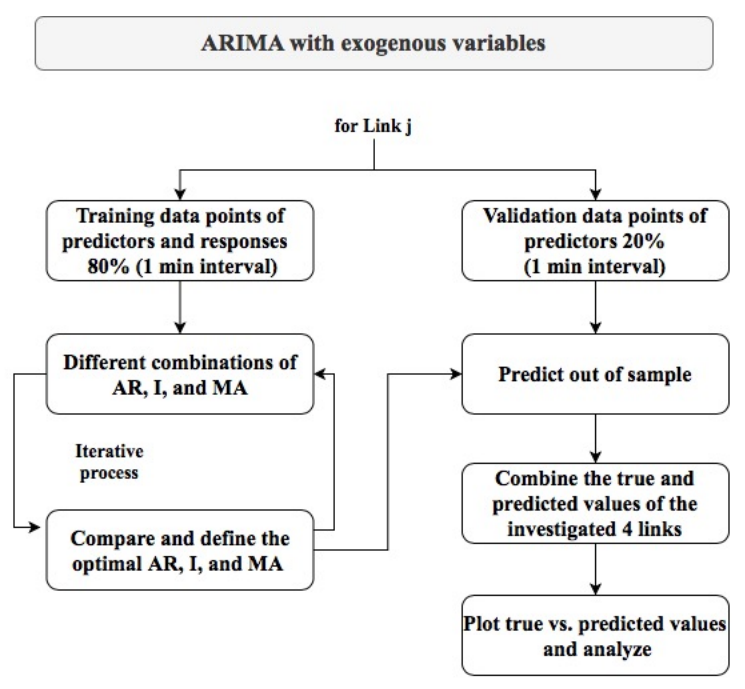

(a)

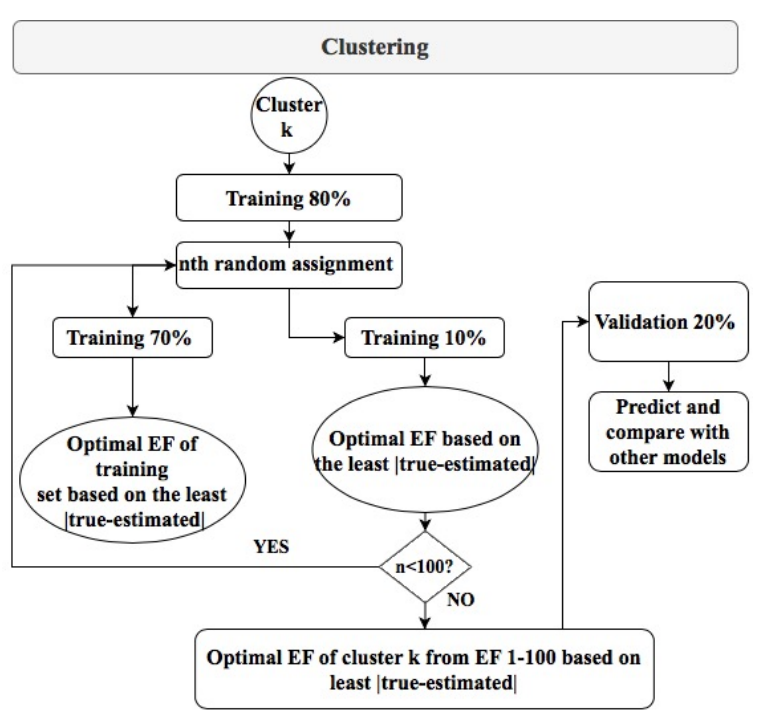

(b)

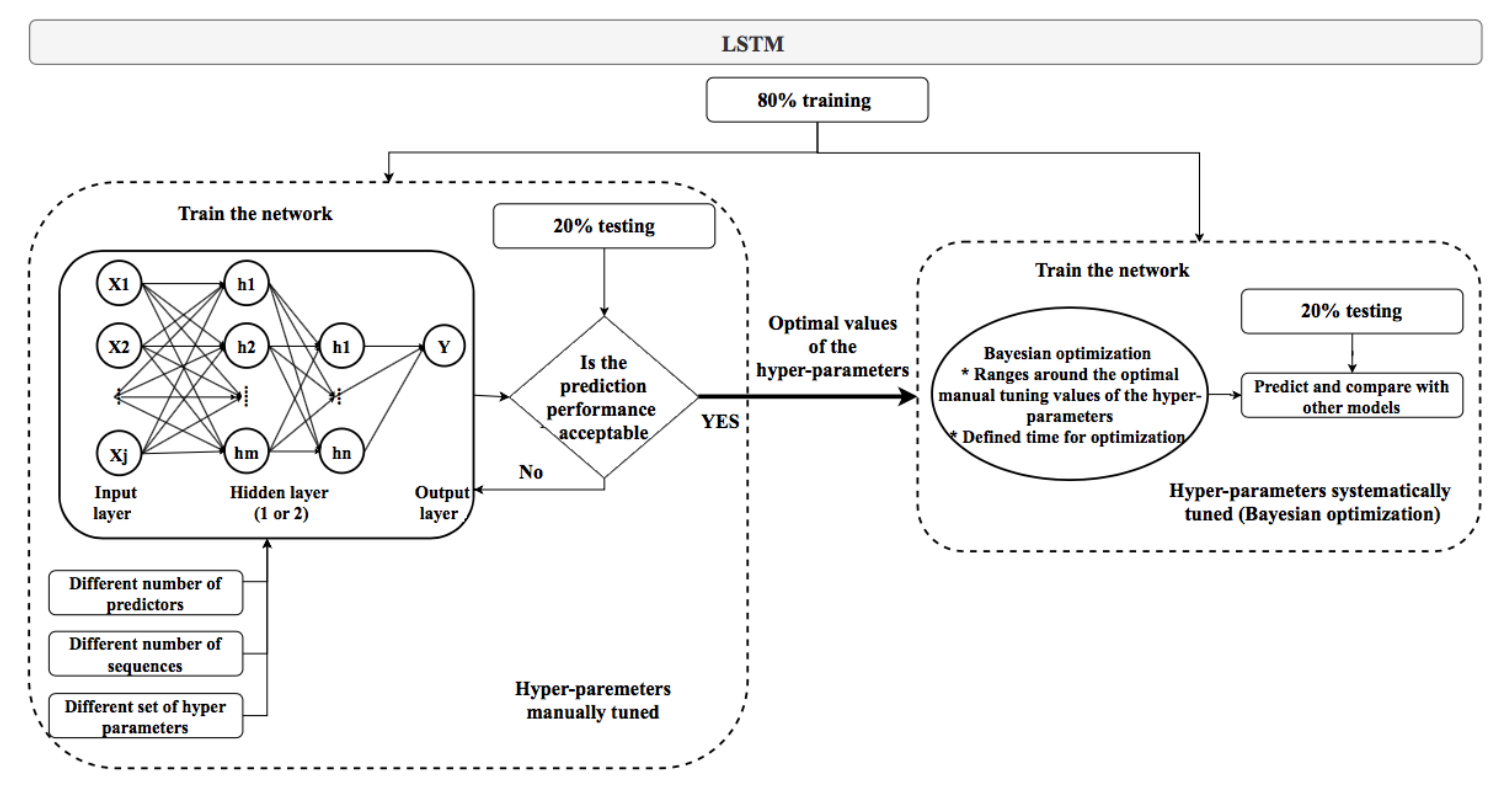

(c)

Figure 6.2 Methodology for a) ARIMA with Exogenous Variables, b) Clustering, and c) LSTM with Exogenous Variables 


\subsection{LSTM with Exogenous Variables}

Recurrent neural network (RNN) is a type of the deep neural networks (DNN). RNN is associated with multiple layers between the input and output layers, unlike the artificial neural network (ANN). The RNN defines the correct mathematical manipulation to give output from an input, whether it is a linear relationship or a non-linear relationship (Aggarwal, 2018). RNNs introduce the concept of memory. The Long-Short Term Memory (LSTM) network is a category of the RNNs, that overcomes the drawback of regular RNNs, the vanishing gradient problem (Amarpuri et al., 2019). The LSTM network has been considered as one of the most powerful RNN architectures where sequential data is involved (Lipton, Berkowitz, and Elkan, 2015). LSTM is associated with three gates (Hochreiter and Schmidhuber, 1997), input, forget, and output following the below Equations 6.3, 6.4, and 6.5 , respectively.

$$
\begin{gathered}
i_{t}=\sigma\left(w_{i}\left[h_{t-1}, x_{t}\right]+b_{i}\right) \\
f_{t}=\sigma\left(w_{f}\left[h_{t-1}, x_{t}\right]+b_{f}\right) \\
o_{t}=\sigma\left(w_{o}\left[h_{t-1}, x_{t}\right]+b_{o}\right)
\end{gathered}
$$

Where $i_{t}$ represents the input gate, $f_{t}$ represents the forget gate, $o_{t}$ represents the output gate, $\sigma$ represents the sigmoid function, $w_{x}$ represents the weight for gate $x$ neurons, $h_{t-1}$ represents the output of the previous LSTM block at time $t-1, x_{t}$ represents the input at current time step $t$, and $b_{x}$ represents biases for respective gates $(x)$. Figure 6.2c presents the

methodology followed of the LSTM application. A ratio of $80 \%-20 \%$ has been considered for training to validation, respectively (Alfaseeh, Tu, et al., 2020). Among the proposed approaches to avoid overfitting, adding drop out layers is an effective and easy way in NNs in general (Srivastava et al., 2014). This is the considered technique for the investigated LSTM networks in this chapter. 
The selection of hyper-parameters of LSTM network has a profound impact on prediction performance (Reimers and Gurevych, 2017; Hutter, Lücke, and Schmidt-Thieme, 2015). It has been proven that increasing the depth of neural network (NNs) in general (Hermans and Schrauwen, 2013; Pascanu et al., 2013) and effectively tuning hyper-parameters (Snoek, Larochelle, and Adams, 2012) may contribute to a desirable effect on prediction performance. In this chapter, one and two LSTM/hidden layers were examined while LSTM model hyperparameters were tuned manually and systematically.

\subsection{Data Collection}

To develop the predictive models at the link level and one minute prediction interval, high resolution data points were required reflecting to traffic and environmental indicators. To fulfill the aforementioned task, the dynamic distributed routing framework (E2ECAV) was used, which employed an agent-based microscopic simulation as illustrated in Section 3.3. The used emission model was MOVES (USEPA, 2015), which has been presented in Section 3.4. The second-by-second traffic and environmental characteristics of every vehicle were utilized to obtain the one minute link level data points. The emission simulator required inputs from the traffic simulator, as shown in Figure 3.3. With time, utilization of the smart cities technologies will increase. This means that the developed predictive models can be retrained on real data and used for prediction purposes.

It is crucial to note that the adoption of a large urban road network, which is downtown Toronto as illustrated in Section 3.5, is associated with a high level of complexity. Nevertheless, it makes the developed predictive models more realistic. Figure 6.3 demonstrates statistical analysis of variables reflecting on traffic conditions and GHG ER (in $\mathrm{CO}_{2 \mathrm{eq}} \mathrm{g} / \mathrm{sec}$ ) on links. From Figure 6.3a, the mode for speed is $40 \mathrm{~km} /$ hour. It is important to note that speed limit of 132 and 18 links in our case study is 60 and $80 \mathrm{~km} /$ hour, respectively. Speed

average in the network based on second-by-second data point is $56.16 \mathrm{~km} /$ hour. However, 
speed varies from 0 to $80 \mathrm{~km} /$ hour. Similarly, density in veh $/ \mathrm{km}$.lane as in Figure $6.3 \mathrm{~b}$ and flow veh/h as in Figure 6.3c are associated with a wide range of values to account for different traffic conditions. Finally, GHG ERs (in $\mathrm{CO}_{2 \mathrm{eq}}$ ) start from less than $1 \mathrm{~g} / \mathrm{sec}$ to more than $5 \mathrm{~g} / \mathrm{sec}$ as in Figure 6.3d. It was noticed that frequency of data points out of [20 60] speed range was significantly lower than values within the aforementioned range. Similarly, frequency of GHG ERs out of [1.5 2.5] g/sec range was dramatically smaller than GHG ERs within the mentioned range.

To develop generic predictive models of GHG ER and speed, a variety of scenarios have been simulated. Different traffic conditions have been mimicked by employing different demand levels and departure time distributions as shown in Table 6.1. For every simulation run, a specific demand level and a distribution to estimate the departure time were deployed.

Travel demand was provided by the Transportation Tomorrow Survey (TTS) (DMG, 2011). The time dependent exogenous demand Origin-Destination (OD) matrices were based on 5 minute intervals from TTS. The demand ranged from 2,437 to 6,988 representing $70 \%$ to $200 \%$ of the 2014 demand provided by the TTS. Different distributions were taken into account for departure time, normal, uniform, and exponential triggering more heterogeneity in traffic conditions. The data have been divided into $80 \%$ training and $20 \%$ validation for clustering LSTM. Training and validation data sets were of 48,652 and 12,159 data points, respectively. For ARIMA model, 4 representative links were considered, which were associated with different characteristics to give an indication of the prediction performance at the network level. Data points of every link have been divided into $80 \%$ to $20 \%$ for training to validation (Alfaseeh, Tu, et al., 2020). The total number of sequences was 83 for ARIMA. Training and validation datasets consisted of 66 and 17 sequences, respectively. 


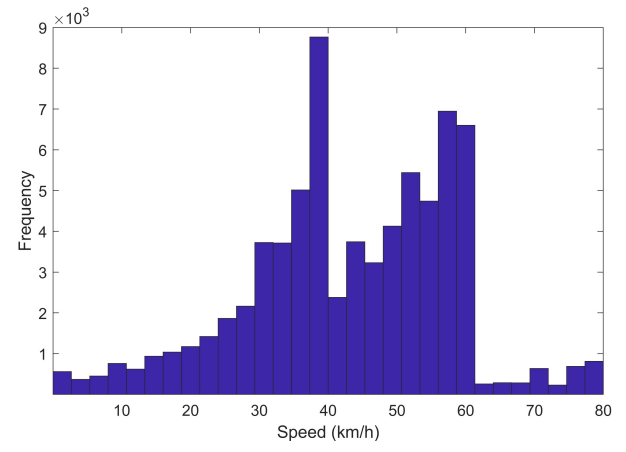

(a)

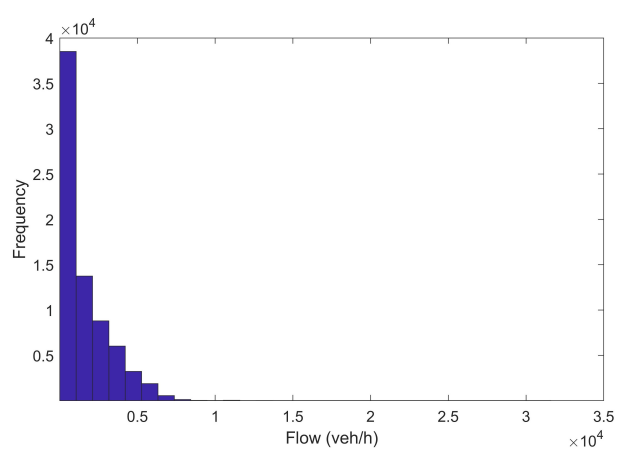

(c)

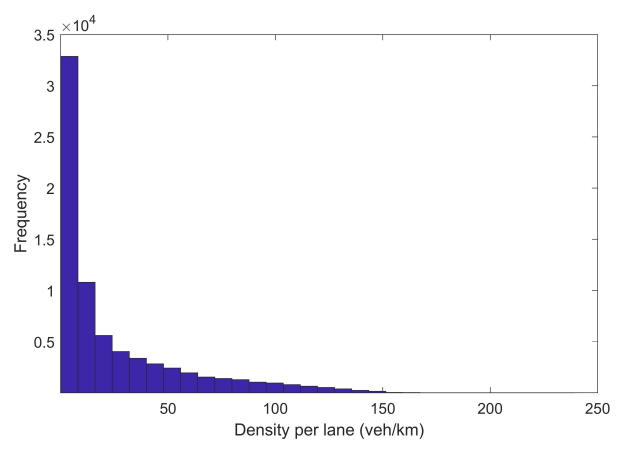

(b)

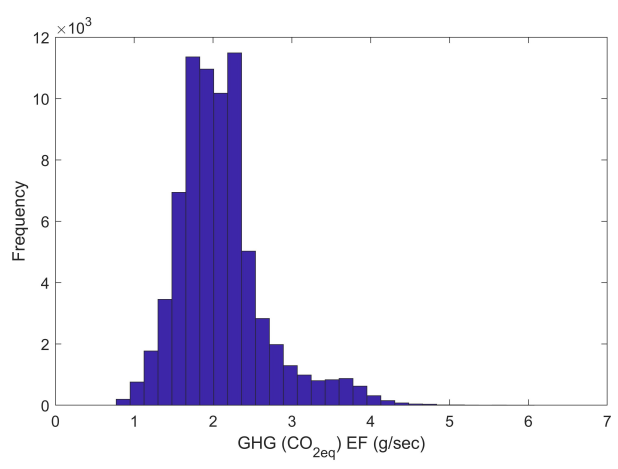

(d)

Figure 6.3 Histogram of a) Speed, b) Density per Lane, c) Flow, and d) GHG ERs (in $\mathrm{CO}_{2 \text { eq }} \mathrm{g} / \mathrm{sec}$ )

Table 6.1 The Considered Scenarios for Data Generation

\begin{tabular}{lcc}
\hline$\%$ of the 2014 demand & No. of Vehicles & Departure time distribution \\
\hline \hline 70 & 2,437 & Exponential, uniform, and normal \\
\hline 100 & 3,477 & Exponential, uniform, and normal \\
\hline 130 & 4,520 & Exponential, uniform, and normal \\
\hline 150 & 5,259 & Exponential \\
\hline 200 & 6,988 & Exponential \\
\hline
\end{tabular}




\subsection{Results and Discussion}

While the three proposed models, ARIMA, clustering, and LSTM, were evaluated for GHG ER prediction, only the best predictive model was deployed for the speed prediction. Before illustrating the results of the predictive models of GHG ER and speed in Sections 6.7.2 and 6.7.3, respectively, a detailed correlation analysis was conducted in Section 6.7.1 for GHG ER and speed, respectively. Four performance indicators were employed for comparison purposes between the predictive models, the correlation coefficient between observed and predicted GHG ERs (in $\mathrm{CO}_{2 \mathrm{eq}} \mathrm{g} / \mathrm{sec}$ ) and speed, fit to the ideal straight curve, $\mathrm{R}^{2}$, and RMSE.

\subsubsection{Correlation Analysis}

This analysis was the reference for choosing the predictors and the number of sequences to develop the predictive models of GHG ER and speed. Five sequences, minutes, represented the period of the correlation investigation with the sixth minute of the variable in concern, GHG ER or speed. The five-minute period was chosen as it was sufficient to reflect on the changes in the traffic and environmental conditions in an urban area. The max link length in the network is around 450 meters. Speed varies from 0 to $80 \mathrm{~km} /$ hour. Under the free flow traffic condition, the maximum travel time required to traverse a link is around 0.8 minute. In this section, the specifications and major findings related to the GHG ER and speed correlation analysis are illustrated, respectively.

\section{Correlation Analysis of GHG ER}

For this analysis, a comprehensive list of variables has been considered not only of the studied links, but also of the up-stream links. In other words, experienced traffic and environmental conditions on in-links at time $(t-1)$ give an indication of what traffic and environmental conditions would be on studied links at time $(t)$. Speed, density, flow, delay (difference between free flow travel time and actual travel time), in-links speed, in-links density, in- 


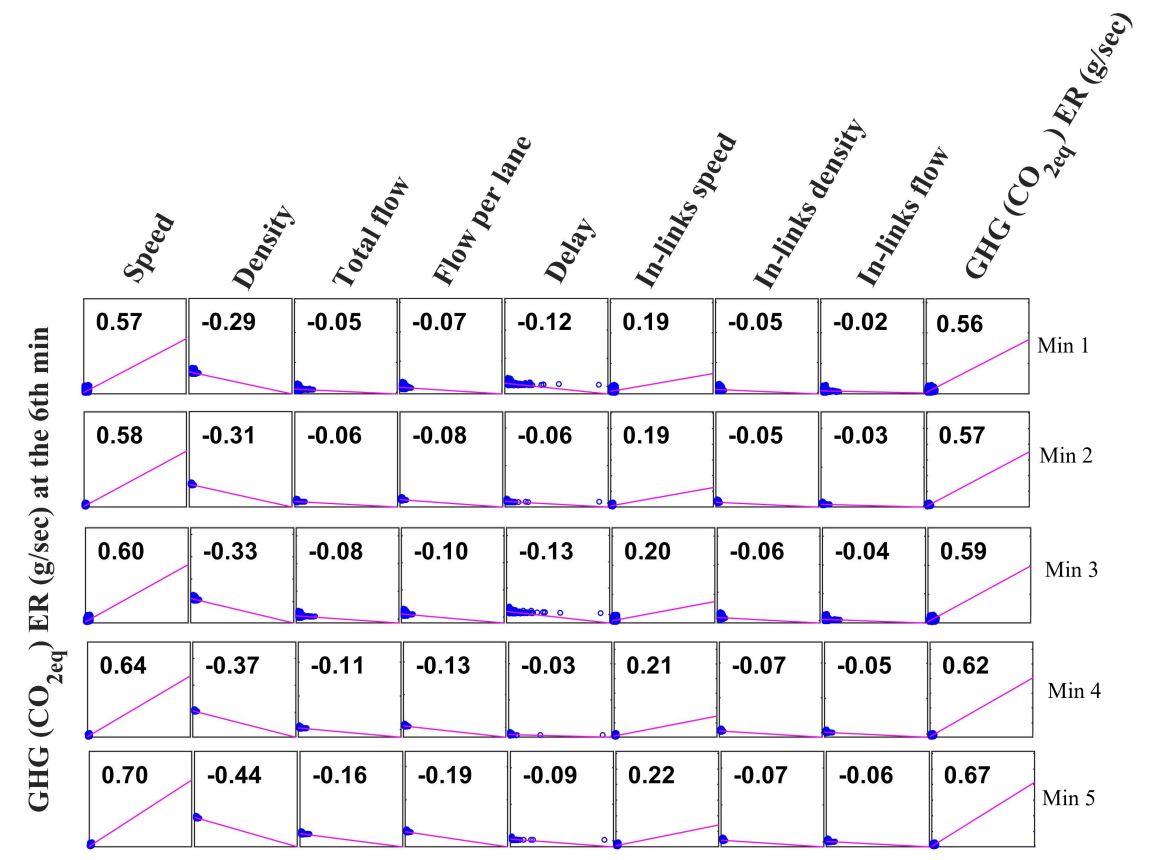

(a)

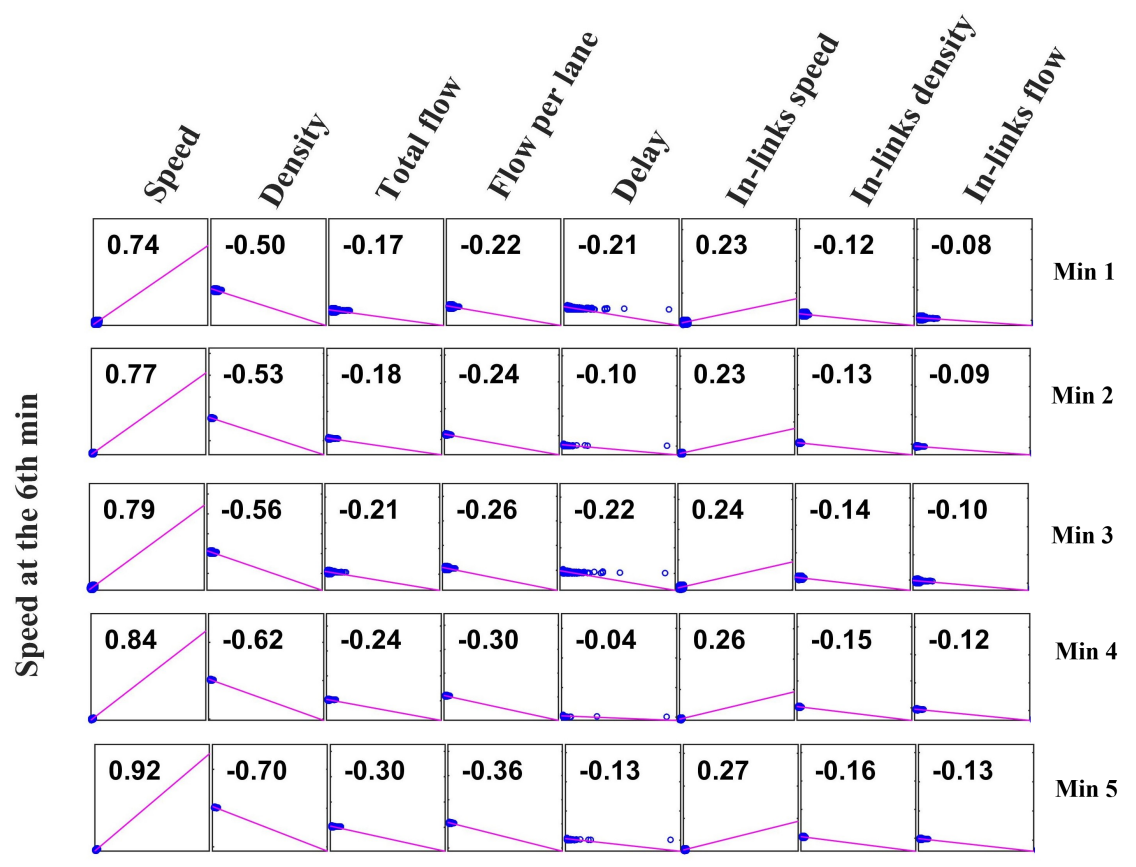

(b)

Figure 6.4 Correlation between the Proposed Variables at Every Minute from 1 to 5 (Top to Bottom) with a) GHG ER and b) Speed at the 6th Minute 
links flow, and GHG ER (in $\mathrm{CO}_{2 \mathrm{eq}} \mathrm{g} / \mathrm{sec}$ ) were the examined variables. Figure 6.4a shows that the absolute value of the correlation coefficient increases between all of the variables except for the delay and the GHG ER (in $\mathrm{CO}_{2 \mathrm{eq}} \mathrm{g} / \mathrm{sec}$ ) at the sixth minute from minute 1 to minute 5 (top to bottom). Speed is the most correlated variables with the GHG ER at the sixth minute, followed by GHG ER (in $\mathrm{CO}_{2 \mathrm{eq}} \mathrm{g} / \mathrm{sec}$ ), density, in-links speed, and the rest of the variables. The dominating role speed plays in GHG ER estimation process, as demonstrated in Equation 3.3, justifies the high correlation (United States Environmental Protection Agency, 2014). The high correlation between GHG ER and density can be explained based on the relationship between speed and density, which are two of the fundamental traffic variables (Papacostas and Prevedouros, 1993). The relationship between speed and density is monotonically decreasing (Papacostas and Prevedouros, 1993). Among the in-links variables, speed is the mostly correlated with GHG ER on the downstream links. The speed on in-links at time $(t-1)$ gives a strong indication of what the speed at time $(t)$ would be on studied links. Different combinations of predictors and number of sequences, minutes, have been assessed for the best outcome.

\section{Correlation Analysis of Speed}

The same logic of the previous subsection is followed here in terms of the number of sequences, minutes, and inclusion of in-links characteristics. In terms of the importance order as shown in Figure 6.4b, speed followed by density, flow per lane, total flow, and in-links speed are the top five highly correlated variables of the previous five sequences with speed at the sixth minute. The high correlation with density is due to the monotonically decreasing relationship between speed and density (Papacostas and Prevedouros, 1993). Among the in-links variables, speed is the most highly correlated one with the speed of a studied link. 


\subsubsection{GHG ER Models}

In this section, the examined predictive GHG ER models, ARIMA, clustering, and LSTM, are presented in order to specify the most suitable model for the employed level of spatial and temporal resolution in this chapter.

\section{ARIMA with Exogenous Variables}

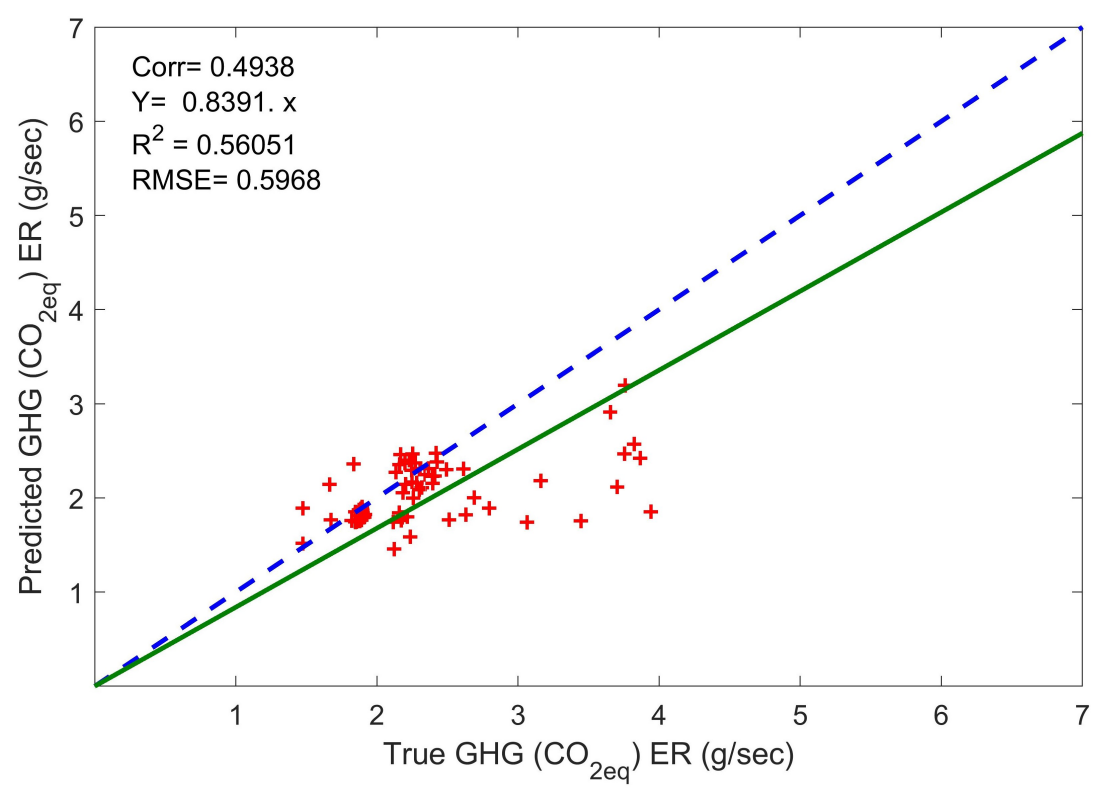

Figure 6.5 Sample Representation (4 Sampled Links) of Network Level Prediction Using ARIMA with Exogenous Variables

The ARIMA model with exogenous variables model is associated with a major drawback related to capturing the non-linearity between variables in concern (G. P. Zhang, 2003). This makes it not suitable for problems of high complexity. Moreover, ARIMA is not scalable. While considering a high spatial level of resolution, every link requires its own model. This can be very tedious and time consuming when the case study is large. However, to compare between the models, a sample of 4 links, which are associated with different characteristics (number of lanes and free flow speed) and conditions (congested and uncongested), were considered. With reference to the predictors adopted, the top three variables, speed, density, 
and GHG ER, were taken. Figure 6.5 shows that GHG ERs between 1.5 and $2 \mathrm{~g} / \mathrm{sec}$ were predicted with a higher level of accuracy compared to GHG ER higher than $2.5 \mathrm{~g} / \mathrm{sec}$. Not capturing the non-linearity between variables can be a justification. The correlation coefficient and $\mathrm{R}^{2}$ of the ARIMA model of the four links are 0.494 and 0.56 , respectively. With regards to the fit to the straight curve, there was underestimation of around $16 \%$ and the RMSE was of $0.597 \mathrm{~g} / \mathrm{sec}$.

\section{Clustering}

The sum of squared error, elbow, approach was employed to define the used number of clusters. Although the optimal number of clusters was five as shown in Figure 6.6, ten and fifteen clusters were examined for further enhancements. Similarly to the ARIMA application, the top three predictors, speed, density, and GHG ER, were employed for this application. The profound limitation of the clustering approach is that the predicted GHG ER was considered as a discrete variable compared to ARIMA model. This means that changes overtime were not captured reliably compare to ARIMA model. Figure 6.7a shows that the predicted values of GHG ERs were either under or overestimated. Values of GHG ERs ranging from 1 to 4 were predicted to be either $1.5,1.8,1.9$, or $2.3(\mathrm{~g} / \mathrm{sec})$. While routing vehicles, optimal routes from origins to destinations were updated at every minute based on link cost. When link cost does not capture fluctuations in GHG ER over time on a studied link, cost will remain the same. For an example, let's say that true value of GHG ERs on a link $k$ at minutes $(t),(t+1)$, and $(t+2)$ are $1.5,2$, and $3 \mathrm{~g} / \mathrm{sec}$, respectively, the predicted value of GHG ER of the three minutes can be the same of around $3.8 \mathrm{~g} / \mathrm{sec}$ while considering 5 clusters. Thus, link GHG cost at the three minutes will remain the same, while in reality the cost is increasing. The priority of choosing this link should change with respect to selecting it to be part of optimal route. For five cluster as shown in Figure 6.7a, the correlation coefficient, $\mathrm{R}^{2}$, and RMSE are $0.71,0.66$, and $0.4(\mathrm{~g} / \mathrm{sec})$, respectively. Increasing the number

of clusters to ten or fifteen in Figures $6.7 \mathrm{~b}$ and $6.7 \mathrm{c}$, respectively, was associated with only a 
slight enhancement to the performance indicators. The correlation coefficient of 10 and 15 clusters has increased by $2.4 \%$ and $3.1 \%$, respectively, compared to 5 clusters. The RMSE of 10 and 15 clusters has decreased by $3.1 \%$ and $3.9 \%$, respectively, compared to 5 clusters.

When ten or fifteen clusters were employed, GHG ER ranging from 2.5 to $4 \mathrm{~g} / \mathrm{sec}$ were either underestimated to be 2.5 or overestimated to be $4 \mathrm{~g} / \mathrm{sec}$. Varying values of predictors, speed, density, and GHG ER, were grouped to be in one, which was associated with one response (GHG ER). In other words, different traffic and environmental conditions can be in the same cluster, which is a drawback when a high level of temporal resolution is urged. While ARIMA lacks the spatial dimension, clustering lacks the temporal dimension. The changes over time were not well reflected on the predicted GHG ER.

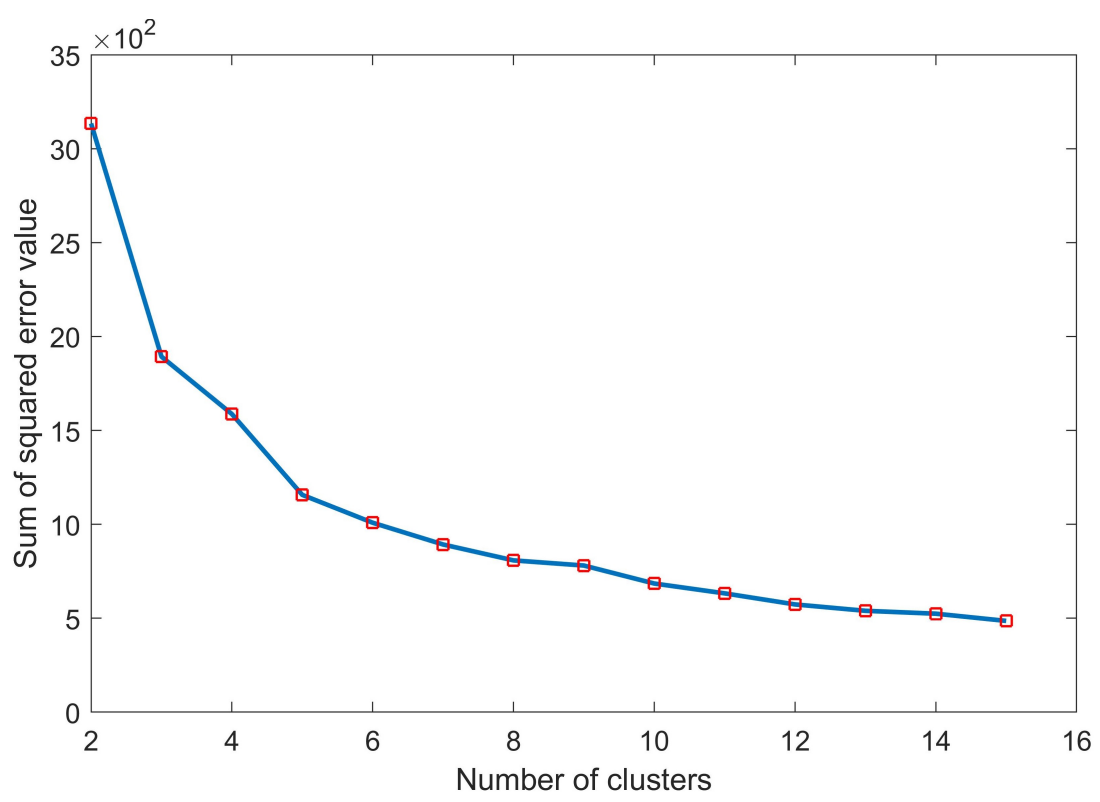

Figure 6.6 Sum of Squared Error (Elbow Method) of 15 Clusters

\section{LSTM with Exogenous Variables Results}

This section demonstrates the results of the proposed LSTM networks in Table 6.2. The first six LSTM networks were related to GHG ER prediction, while the last two LSTM networks were related to speed prediction. The results are discussed in the following subsections, 


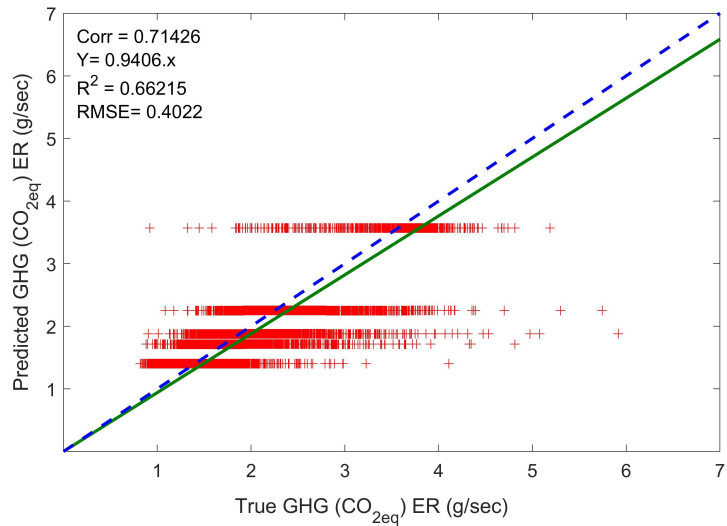

(a)

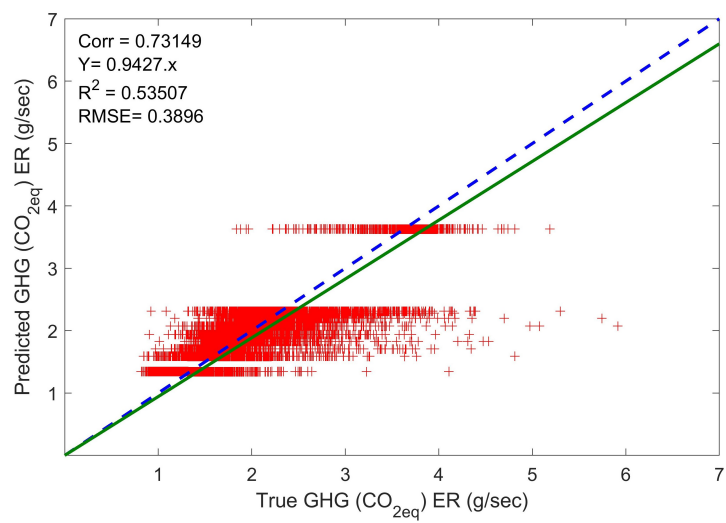

(b)

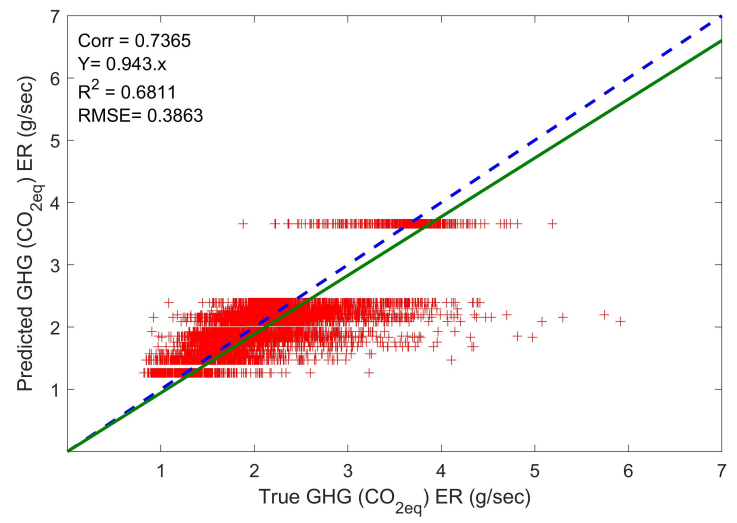

(c)

Figure 6.7 True vs. Predicted GHG ERs (in $\mathrm{CO}_{2 \mathrm{eq}} \mathrm{g} / \mathrm{sec}$ ) of Clustering Where: a) is for 5 Clusters, b) is for 10 Clusters, and c) is for 15 Clusters

respectively. Each of the LSTM networks was tuned manually (indexed with ${ }_{m}$ ) and systematically (indexed with ${ }_{b}$ ) to illustrate the importance of the latter tuning approach. The Bayesian optimization (Gelbart, Snoek, and Adams, 2014) was utilized for the systematic tuning.

To define the best LSTM predictive network, a long list of options with regards to selection of predictors, number of sequences, and choice of hyper-parameters has been considered (Reimers and Gurevych, 2017; Hutter, Lücke, and Schmidt-Thieme, 2015). It is crucial to note that increasing the depth of NNs (Hermans and Schrauwen, 2013; Pascanu et al., 2013) and effectively tuning the hyper-parameters (Snoek, Larochelle, and Adams, 2012) may 
Table 6.2 Trained LSTM Network and their specifications

\begin{tabular}{|c|c|c|c|c|c|c|}
\hline $\begin{array}{l}\text { Model } \\
\text { ID }\end{array}$ & Predictors & Response & $\begin{array}{c}\text { No. of } \\
\text { sequences/ } \\
\text { minutes }\end{array}$ & $\begin{array}{l}\text { No. of } \\
\text { LSTM } \\
\text { layers }\end{array}$ & $\begin{array}{l}\text { Sequence } \\
\text { interval }\end{array}$ & $\begin{array}{c}\text { Hyper-param. } \\
\text { tuning } \\
\text { approach }\end{array}$ \\
\hline $\mathrm{LSTM}_{m}$ & $\begin{array}{l}\text { Speed, density, } \\
\text { and GHG ER }\end{array}$ & GHG ER & 3 & 1 & $1 \mathrm{~min}$ & Manual \\
\hline $\mathrm{LSTM}_{b}$ & $\begin{array}{l}\text { Speed, density, } \\
\text { and GHG ER }\end{array}$ & GHG ER & 3 & 1 & $1 \mathrm{~min}$ & Bayesian \\
\hline $\mathrm{LSTM} 2_{m}$ & $\begin{array}{l}\text { Speed, density, GHG ER, } \\
\text { and in-links speed }\end{array}$ & GHG ER & 3 & 1 & $1 \mathrm{~min}$ & Manual \\
\hline $\mathrm{LSTM}_{b}$ & $\begin{array}{l}\text { Speed, density, GHG ER, } \\
\text { and in-links speed }\end{array}$ & GHG ER & 3 & 1 & $1 \mathrm{~min}$ & Bayesian \\
\hline $\mathrm{LSTM}_{m}$ & $\begin{array}{c}\text { Speed, density, GHG ER, } \\
\text { and in-links speed }\end{array}$ & GHG ER & 3 & 2 & $1 \mathrm{~min}$ & Manual \\
\hline $\mathrm{LSTM}_{b}$ & $\begin{array}{l}\text { Speed, density, GHG ER, } \\
\text { and in-links speed }\end{array}$ & GHG ER & 3 & 2 & $1 \mathrm{~min}$ & Bayesian \\
\hline $\mathrm{LSTM} 4_{m}$ & $\begin{array}{l}\text { Speed, density, } \\
\text { and in-links speed }\end{array}$ & Speed & 3 & 1 & $1 \mathrm{~min}$ & Manual \\
\hline $\mathrm{LSTM}_{b}$ & $\begin{array}{l}\text { Speed, density, } \\
\text { and in-links speed }\end{array}$ & Speed & 3 & 2 & $1 \mathrm{~min}$ & Bayesian \\
\hline
\end{tabular}

contribute to in favour outcome. Hence, one and two hidden layers were examined and hyper-parameters were tuned following two stages: manual and systematic (Bayesian optimization) tuning. Two solvers were considered: the stochastic gradient descent with momentum (sgdm) (Robert, 2014) and adaptive moment estimation (Adam) methods (Kingma and Ba, 2014). The sgdm adds a momentum term to the parameter update to reduce oscillation associated with the standard stochastic gradient descent solver along the path of steepest de- 
scent towards the optimal solution (Robert, 2014). Adam is derived from adaptive moment estimation. It adopts different learning rates for the parameters unlike sgdm, but uses a momentum term like the sgdm to further improve network training (Kingma and Ba, 2014). The hyper-parameters considered are as follows: the initial learning rate, momentum, max epochs, learning rate drop factor, learning rate drop period, number of hidden units of the first LSTM (hidden) layer, and the number of hidden units of the second (LSTM) layer when used. After examining different number of sequences, three minute period was the optimal and was adopted for the predictive LSTM networks. The manual tuning helped narrowing the search range of the hyper-parameters in concern. Then came the systematic tuning, using the Bayesian optimization and based on the best manually tuned hyper-parameters for further improvements. The results of the whole LSTM networks, whether manually or systematically tuned, predicting GHG ER and speed are demonstrated in Table 6.3.

With respect to the LSTM networks and their specifications as shown in Table 6.2, the three most important variables were used in LSTM1. The LSTM1 was used for comparison purposes with the ARIMA, and clustering models. LSTM2 considered the variables of LSTM1 in addition to the most important in-link variable, which is the in-link speed. Finally, LSTM3 considered the predictors or LSTM2, but employed an additional hidden layer for further enhancements. Three sequences have been found to be the best sequence (Alfaseeh, Tu, et al., 2020). Figures $6.8 \mathrm{a}$ and $6.8 \mathrm{~b}$ demonstrate the results of the $\mathrm{LSTM} 1_{m}$ and $\mathrm{LSTM}_{b}$ networks, respectively. A noticeable enhancement in the prediction performance is associated with the latter LSTM network. A reduction in the RMSE of $17 \%$ is observed compared to $\mathrm{LSTM}_{m}$. A slight increase in the correlation coefficient and $\mathrm{R}^{2}$ of $2 \%$ and $3 \%$, respectively is noticed in $\mathrm{LSTM}_{b}$ compared to $\mathrm{LSTM} 1_{m}$. This assures that systematically tuning the hyper-parameters contributed to a better prediction performance. The LSTM1 $1_{b}$ underestimated the GHG ER by $3 \%$, while the $\mathrm{LSTM}_{m}$ overestimated by $7 \%$. The $\mathrm{LSTM}_{b}$ outperformed both ARIMA and the best clustering model in terms of the whole performance indicators. The correlation coefficient of $\mathrm{LSTM}_{b}$ was increased by $52 \%$ and $1.4 \%$ compared 
to the ARIMA and clustering of 15 clusters, respectively. The $\mathrm{R}^{2}$ of $\mathrm{LSTM}_{b}$ is $23 \%$ and $1.3 \%$ higher than ARIMA and the best model of clustering, respectively. Finally, the RMSE of $\mathrm{LSTM}_{b}$ has improved by $37 \%$ and $3 \%$ compared to the ARIMA and the best model of clustering, respectively. While the ARIMA model is not scalable, LSTM can be applied at a network level regardless of the characteristics of links, speed, number of lanes, directions. In other words, LSTM is more generic compared to ARIMA. It is worth mentioning that the prediction performance of ARIMA is link based and was subject to the level of congestion on a studied link. For two uncongested links, RMSE was 0.0718 and $0.0054 \mathrm{~g} / \mathrm{sec}$. On the other hand in the case of two congested links, RMSE of ARIMA was 0.95 and $0.4494 \mathrm{~g} / \mathrm{sec}$. On the other hand, LSTM was more stable regardless of the traffic condition on links. RMSE ranged from 0.3577 to 0.4494 whatever the traffic condition on the studied links was. The major drawback of considering predicted GHG ER as a discrete variable in clustering was overcome by LSTM. In LSTM application, GHG ER is a continuous variable and depends only on the most representative predictors of the last three sequences in our application. Unlike clustering that groups traffic and environmental variables into clusters, LSTM provides a response for every set of predictors.

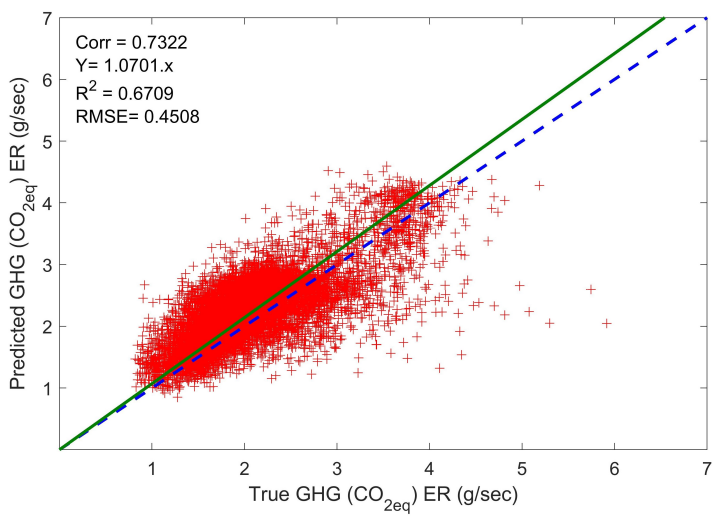

(a)

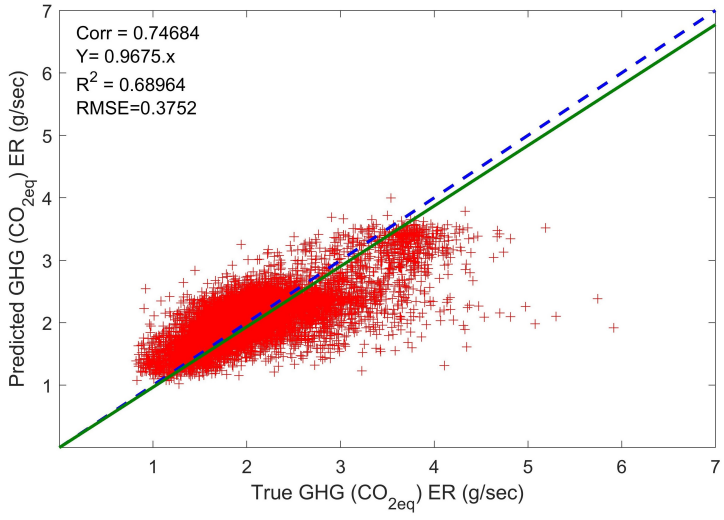

(b)

Figure 6.8 True vs. Predicted GHG ERs (in $\mathrm{CO}_{2 \mathrm{eq}} \mathrm{g} / \mathrm{sec}$ ) of a) $\mathrm{LSTM}_{m}$ and b) $\mathrm{LSTM}_{b}$

It can be concluded that LSTM was the best predictive model among the investigated 
Table 6.3 Performance Indicators of the Trained LSTM Networks

\begin{tabular}{ccccc}
\hline $\begin{array}{c}\text { Model } \\
\text { ID }\end{array}$ & $\begin{array}{c}\text { Correlation } \\
\text { coefficient }\end{array}$ & $\mathrm{R}^{2}$ & $\begin{array}{c}\text { Linear } \\
\text { fit }\end{array}$ & RMSE \\
\hline \hline $\mathrm{LSTM}_{m}$ & 0.732 & 0.671 & $\mathrm{Y}=1.07 \times$ & 0.451 \\
\hline $\mathrm{LSTM}_{b}$ & 0.747 & 0.690 & $\mathrm{Y}=0.97 \times$ & 0.376 \\
\hline $\mathrm{LSTM}_{m}$ & 0.733 & 0.672 & $\mathrm{Y}=1.06 \mathrm{x}$ & 0.445 \\
\hline $\mathrm{LSTM}_{b}$ & 0.748 & 0.691 & $\mathrm{Y}=0.97 \times$ & 0.374 \\
\hline $\mathrm{LSTM}_{m}$ & 0.702 & 0.573 & $\mathrm{Y}=0.89 \times$ & 0.491 \\
\hline $\mathrm{LSTM}_{b}$ & 0.767 & 0.704 & $\mathrm{Y}=0.97 \times$ & 0.363 \\
\hline $\mathrm{LSTM}_{m}$ & 0.888 & 0.822 & $\mathrm{Y}=0.97 \times$ & 7.013 \\
\hline $\mathrm{LSTM}_{b}$ & 0.925 & 0.866 & $\mathrm{Y}=0.97 \times$ & 5.95 \\
\hline
\end{tabular}

ones due to its scaleability and consideration of temporal changes and non-linearity between variables in concern. Hence, LSTM2 and LSTM3 were applied for further improvements in the GHG ER prediction performance.

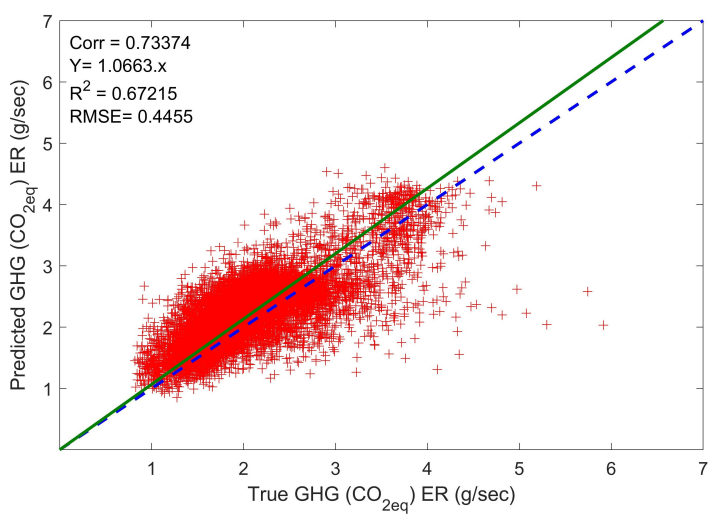

(a)

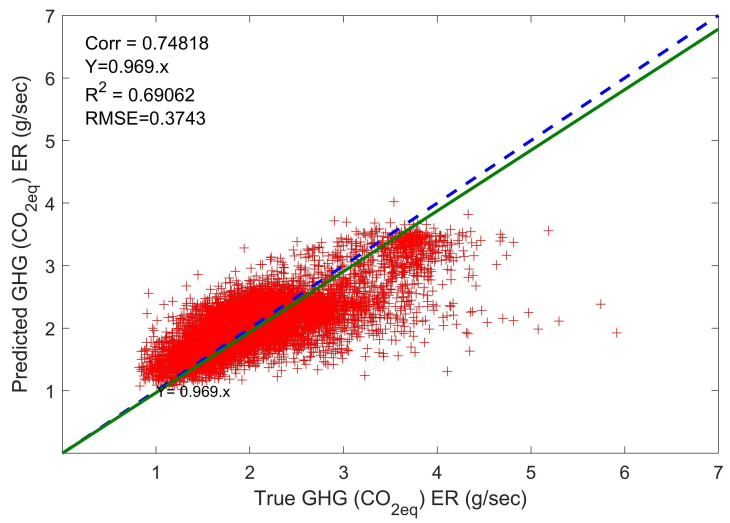

(b)

Figure 6.9 True vs. Predicted GHG ERs (in $\mathrm{CO}_{2 \mathrm{eq}} \mathrm{g} / \mathrm{sec}$ ) of a) $\mathrm{LSTM}_{m}$ and b) $\mathrm{LSTM} 2_{b}$

The performance of $\mathrm{LSTM}_{m}$ and $\mathrm{LSTM}_{b}$ in Figures 6.9 is comparable to $\mathrm{LSTM} 1_{m}$ and 
$\mathrm{LSTM}_{b}$ in Figure 6.8. Including in-links speed has not brought in further enhancement. Nevertheless, considering the most correlated indicator with GHG ER of in-links is in favour and gives a higher consideration to spatial dimension.

Finally, to increase the prediction accuracy, a deeper LSTM network was used for the same predictors of LSTM2. $\mathrm{LSTM}_{b}$ is associated with a significant reduction in the RMSE of $26 \%$ as shown in Figure 6.10b compared to $\mathrm{LSTM}_{m}$ as in Figure 6.10a. This asserts that increasing the depth and systematically tuning the hyper-parameters of LSTM networks is in favour. $\mathrm{LSTM}_{b}$ is associated with a reduction in the RMSE of $19 \%$ compared to $\mathrm{LSTM} 1_{m}$. The LSTM3 $3_{b}$ was used for the anticipatory routing application in Chapter 7 when GHG was part of the optimization process. The accuracy of the predictive model is important. In the case of single or multi-objective eco-routing, the low accuracy would trigger over or underestimation of GHG cost of links. This would have an undesirable effect on the efficiency of routing by not reflecting on the real traffic and environmental states reliably. When link GHG cost is based on average speed, over or underestimation of link GHG cost would be the outcome as found in Alfaseeh and Farooq (2020b).f

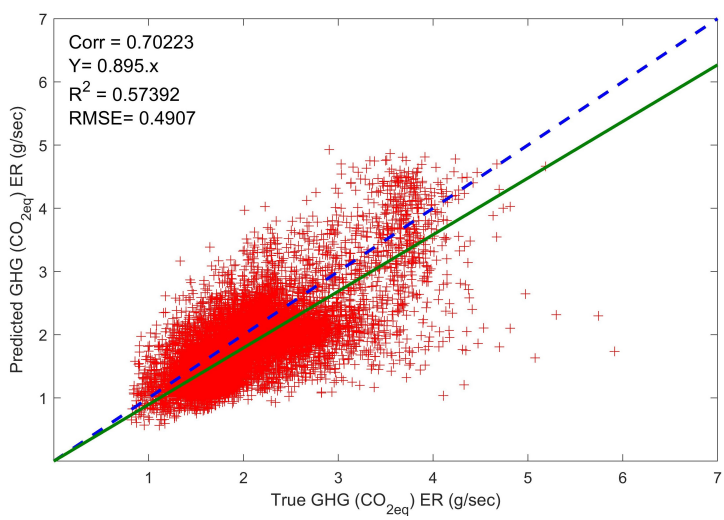

(a)

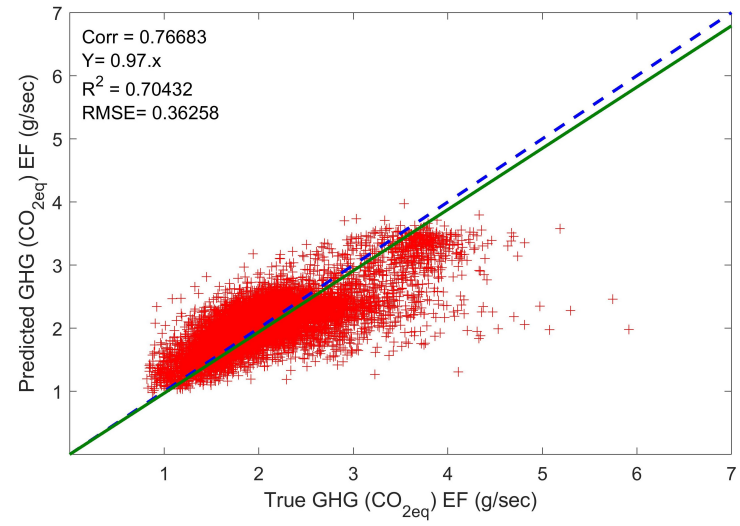

(b)

Figure 6.10 True vs. Predicted GHG ERs (in $\mathrm{CO}_{2 \mathrm{eq}} \mathrm{g} / \mathrm{sec}$ ) of a) $\mathrm{LSTM}_{m}$ and b) $\mathrm{LSTM}_{b}$

The common feature in the $\mathrm{LSTM}_{b}, \mathrm{LSTM} 2_{b}$, and $\mathrm{LSTM}_{b}$ is that GHG ERs over 4 $\mathrm{g} / \mathrm{sec}$ were not predicted with a high level of accuracy. This can be explained by referring 
to the histograms in Figure 6.3. It can be noticed that the frequency of links of GHG ER greater than 4 (gram/second) is very low. Hence, generating more data points to reflect on this condition may bring in higher accuracy of prediction when GHG ER is higher than 4 $(\mathrm{g} / \mathrm{sec})$.

\subsubsection{Speed Model}

As LSTM has outperformed the assessed other two predictive models, namely ARIMA and clustering, LSTM was the predictive model utilized to predict speed on links. The last two networks in Table 6.2 were for speed prediction. Their specifications in terms of the predictors, number of sequences, number of LSTM layers, sequence interval, and the method of tuning the hyper-parameters are demonstrated in the same table.

\section{LSTM Networks of Speed}

After examining different sets of predictors and number of sequences based on the conducted correlation analysis in Section 6.7.1, speed, density, and in-links speed were the variables employed of the last three sequences, minutes. The developed $\mathrm{LSTM} 4_{m}$ and $\mathrm{LSTM} 4_{b}$ were associated with one and two hidden layers, respectively. Figure 6.11b shows a significant reduction in the RMSE of $15 \%$ compared to the $\mathrm{LSTM}_{m}$ as in Figure 6.11a.

This emphasizes on the importance of increasing the depth of LSTM network and systematically tuning the network hyper-parameters. Every layer in a deep network processes part of the task. It develops its representation and passes it to the next layer. The next layer employs the outcome of the previous layer and processes another part of the task till the last hidden layer, which gives the outcome (Hermans and Schrauwen, 2013). Adding hidden layers to an LSTM network is like representing the problem at different time scales (Pascanu et al., 2013). While the fit to the linear straight curve is identical of $\mathrm{LSTM}_{m}$ and $\mathrm{LSTM}_{b}$, the correlation coefficient and $\mathrm{R}^{2}$ increase by $4 \%$ and $5 \%$ in the latter case, 


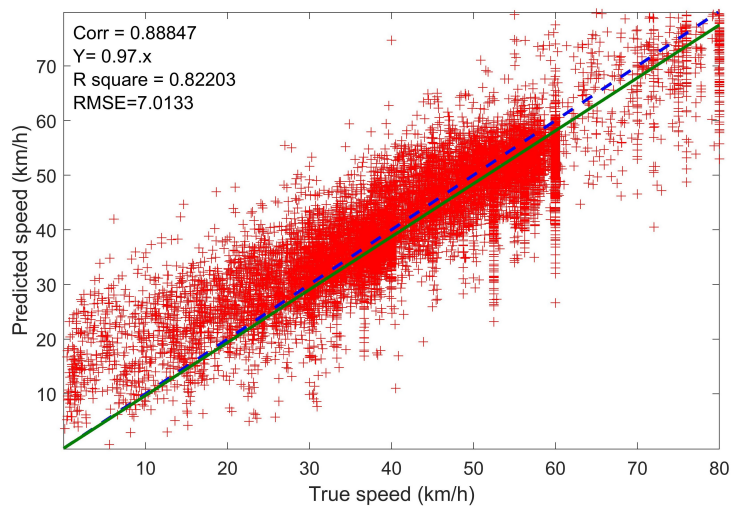

(a)

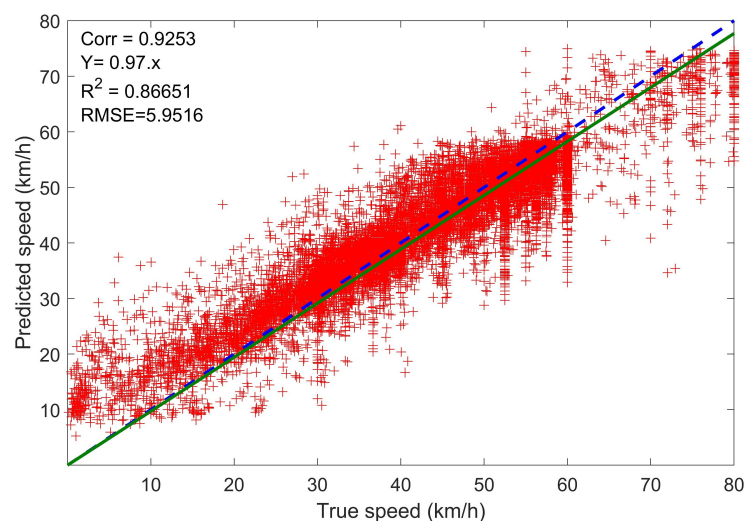

(b)

Figure 6.11 True vs. Predicted Speed $(\mathrm{km} / \mathrm{h})$ of a) $\mathrm{LSTM}_{m}$ and b) $\mathrm{LSTM}_{b}$

which was triggered by the increased depth of the network. It is important to note that the speed prediction accuracy in significantly higher than the case of GHG ER prediction as illustrated in Section 6.7.2. Specifically, the correlation coefficient and $\mathrm{R}^{2}$ of $\mathrm{LSTM}_{b}$ is higher by $21 \%$ and $23 \%$ compared to the best LSTM predictive network, LSTM $3_{b}$. This is due to the complicated non-linear relationship between GHG ER and the most correlated traffic variable, speed. The quasi-convex relationship (Djavadian, Tu, et al., 2020) between GHG ER and speed makes prediction more challenging. On the other hand, speed and density for example have a monotonically decreasing relationship, which makes the prediction more straight forward compared to GHG ER. The LSTM4 $4_{b}$ network was used for the anticipatory routing in Chapter 7 when TT or GHG were part of the optimization process.

\subsection{Concluding Remarks}

Due to the significant negative impact of transportation systems on the environment, there is a necessity to take advantage of ICT advancements to minimize the produced GHG emissions. The use of smart cities technologies will increase in the near future and make challenging research topics achievable, such as prediction, due to the availability of real-time data. Efficiently predicting traffic and environmental indicators will absolutely improve the network 
performance in different ways. Routing, which is one of the potential applications that will benefit from prediction, has a direct impact on both traffic and environmental characteristics. Thus, switching from the myopic, in Chapter 5, to the anticipatory routing, in Chapter 7, was dependent on the prediction quality.

In this chapter, three types of predictive models, ARIMA, clustering, and LSTM, were developed and a comparison was conducted to specify the most suitable model for the defined setting. Unlike the previous related work in the literature, two deep learning based predictive models have been developed while considering a large congested urban network and high level of spatial (link level), and temporal (1 minute) resolution. For a robust application, a comprehensive correlation analysis of both GHG ER and speed was applied. Not only the links indicators, but also in-links characteristics were taken into account. The essential findings of this chapter are as follows. The LSTM network outperformed both ARIMA and the best clustering model in terms of the whole performance indicators. The RMSE of $\mathrm{LSTM}_{b}$ of GHG ER prediction, which considered the top three highly correlated variables, has been reduced by $37 \%$ and $3 \%$ compared to the ARIMA and the best case of clustering, respectively. The LSTM overcame the shortcomings of the other two models investigated. While ARIMA is not scalable, LSTM can be used at a network level. Unlike clustering that considered GHG ER as a discrete variable, LSTM considered GHG ER as a continuous variable and was able to capture changes over time. In clustering, different traffic conditions were grouped together and every group was associated with one response. This means that changes in network status were not captured well, which affected negatively the link cost estimation process.

LSTM took into account the spatial and temporal dimensions efficiently reflecting on changes in the traffic conditions. With respect to the LSTM application, the results showed that increasing the depth of the LSTM network was in favour and triggered significant enhancements in terms of the whole prediction performance indicators, correlation coefficient, $\mathrm{R}^{2}$, linear fit, and RMSE. Although it was noticed that incorporating the in-links most highly 
correlated variable with predicted GHG ER did not bring in improvements, keeping the inlinks highly correlated variable took into account the spatial dimension more efficiently. Not to forget that systematically tuning the LSTM network introduced noticeable enhancement compared to manually tuning the same network. The best LSTM GHG ER and speed predictive models were used to develop the anticipatory routing strategies in Chapter 7, which was compared to the conventional myopic routing strategies in Chapter 5. 


\section{Chapter Seven}

\section{Anticipatory Eco-Routing}

This chapter has resulted in the following publication:

1. Alfaseeh, Lama and Bilal Farooq. (2020). "Deep Learning Based Anticipatory MultiObjective Eco-Routing Strategies for Connected and Automated Vehicles". arXiv preprint arXiv:2006.16472 (under-review in Frontiers in Future Transportation). 


\subsection{Introduction}

The existing myopic routing depends on the previous time step, but anticipatory routing estimates the link cost based on predicted values from the current time step. Anticipatory routing takes into account the evolution of the traffic and environmental conditions, which makes it proactive compared to the myopic reactive routing strategy (Bottom, 2000). The link cost, such as travel time and GHG emissions, in the case of myopic routing is taken at time step $(t-1)$. In the case of anticipatory routing, the cost of the links is at time step $(t)$. The future values are based on either historical data, traffic simulations, or predictive models (Bottom, 2000). Anticipatory routing is proposed as a potential tool to alleviate congestion and outperform the myopic routing strategy. Frameworks have been developed for real-time dynamic anticipatory routing applications, such as DYNASMART, MITSIM, and DynaMIT (Ben-Akiva et al., 2001; Bottom, 2000; Mahmassani, 1994) in a centralized routing framework, while the variable message sign (VMS) was the tool for disseminating information on the best routes to drivers. In previous studies on anticipatory routing, simple prediction models were employed to predict traffic and/or environmental optimized variables while routing vehicles to their destinations. The predicted travel time was based on either previous iterations (Ben-Akiva et al., 2001; Kaufman, Smith, and Wunderlich, 1991), linear relationships between speed and density (Pan et al., 2013), or travel times when vehicles were exiting a studied link (Ben-Akiva et al., 2001).

In this chapter, the developed sophisticated predictive models in Chapter 6 were deployed to apply the anticipatory multi-objective eco-routing. The advancements in ICT, connected automated vehicles and sensing technology, were exploited for a better performing transportation system from traffic and environmental perspectives. Similar to Chapter 5, three routing objectives were considered in this chapter: TT, GHG, and TT \& GHG. A detailed comparison was conducted between the anticipatory and myopic routing strategies at the link and network levels to comprehend respectively the local and global impact of both routing 
types.

Figure 7.1 illustrates the framework of this chapter and its relationship with other chapters in this dissertation. This chapter is organized as follows. A brief literature review is presented in Section 7.2 on anticipatory routing frameworks and applications of anticipatory routing. The specifications of the traffic and emission models employed in this chapter are presented in Section 7.3. The details of the case study for the anticipatory multi-objective eco-routing application are demonstrated in Section 7.4. The crucial highlights of the used predictive models of the optimized variables are illustrated in Section 7.5. Section 7.6 includes a detailed analysis of the anticipatory multi-objective eco-routing strategies in addition to a comparison with the myopic strategies. Finally, the concluding remarks are presented in Section 7.7.

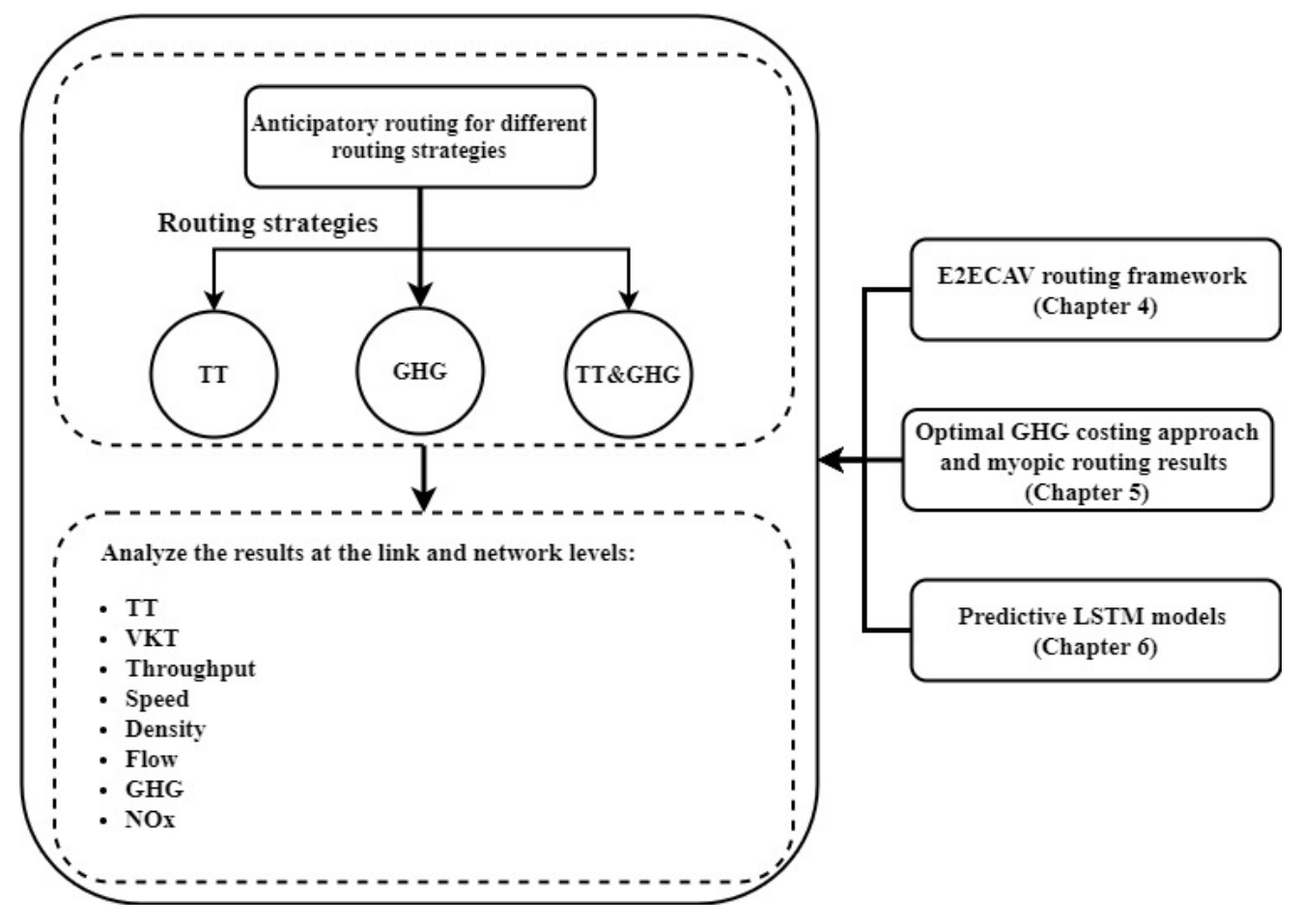

Figure 7.1 Chapter Seven Framework 


\section{$7.2 \quad$ Literature}

A limited number of studies are related to anticipatory routing. Limitations were found with respect to the scale of the case studies; included routing objectives; routing framework; origin-destination (OD) pairs; aggregation level of traffic and emission models; and predictive models.

Kaufman, Smith, and Wunderlich (1991) applied anticipatory routing in a small case study, and travel time was the objective. The predicted travel time was based on the travel time when exiting a link. However, they found that this prediction approach was associated with inconsistencies of predicted values. Hence, they adopted the time when vehicles exited the links of a previous simulation iteration. A prediction interval of one minute was used. Consistency of predicted values was achieved by comparing the predicted travel time with the latest experienced travel time while a tolerance factor was employed. From the results, the authors found that the anticipatory routing contributed to reductions in travel times for drivers and the overall network at market penetration rates up to $50 \%$ of anticipatory route guidance equipped vehicles (Kaufman, Smith, and Wunderlich, 1991).

Mahmassani (1994) proposed a simulation-assignment framework, which is DYNASMART. It was stated that it was capable of applying the anticipatory routing if predictive functions were provided. The authors urged the need for reliable predictive models to help switch from myopic to anticipatory routing for a better performing transportation system.

Bottom (2000) developed a framework for anticipatory routing that included three fixedpoint formulations for guidance consistency with deterministic, quasi-deterministic and fully stochastic models. The author adopted a simple 14-link network with a single OD pair and eleven OD paths. The author developed a simulator for the three major variables. The three variables considered were all time-dependent and consisted of network conditions, path splits, and guidance messages. The major relationships between the fundamental variables were defined by using three maps: the network loading map, which adopted the path splits 
to specify the network conditions; the guidance map, which used the network conditions to define the corresponding guidance messages; and the routing map, which used guidance messages to apply path splits. Predictive models were not used by the author, and it was assumed that the data were collected and processed into the inputs required by a traffic prediction model (Bottom, 2000). The VMS was employed to provide vehicles with the best route (Bottom, 2000). When the best route was defined, it was not guaranteed that drivers would comply and follow the route. Hence, the author incorporated a simple approach that highlighted the main issues of the behavior of drivers in the developed framework. The path choice was defined based on the logit model. The framework was tested with different problems. The stochasticity in the outputs was the major performance indicator. It was found that compliance by the drivers was an essential element that contributed to the stochasticity in the path flow per time period, which affected the flow evolution in the network (Bottom, 2000).

Ben-Akiva et al. (2001) proposed DynaMIT, which is a real-time system that can be used to generate guidance for drivers. Off-line and real-time information were adopted. The off-line data were the historical network conditions that were used for estimating the state. The real-time data were obtained from the control system. The OD pairs were defined at an aggregated level, while the response of the drivers was captured at a disaggregated level. Thus, the simulation tools within DynaMIT combined microscopic and macroscopic models, which made them mesoscopic simulators. Two simulation tools were used: the demand simulator and supply simulator. The demand simulator estimated and forecasted the origin-destination (OD) flow, departure time of drivers, mode, and route choice. While the supply simulator directly simulated the interactions between the demand and supply within the network. Travel time was the only routing objective. The predicted travel time for the vehicles was a function of the travel time experienced from the previous simulation iteration. Speed on the links was estimated based on its linear relationship with density on the same link. The VMS was used to inform drivers about the best route. From this analysis, 
the authors found that anticipatory routing was promising as it contributed to reducing the travel time of vehicles (Ben-Akiva et al., 2001).

Claes (2015) developed an anticipatory traveller information system (AntTIS), which was modelled as a multiagent system (MAS). MASs were software systems that consisted of multiple autonomous software agents. The author got inspired by the ant colony concept. The overall functionality of the software system was achieved based on interactions between the individual agents. To mitigate the complexity of coordinating between the large number of agents interacting within a large scale network, AntTIS employed a technique called the delegate multiagent system (dMAS). The goal of the proposed AntTIS was to delay congested buildups and to assist drivers/road users make reliable decisions on the shortest paths towards their destinations. The system mainly collected information from road users about the road links they intended to use. Then, the traffic condition was predicted accordingly. Delegate multi-agent systems were used to collect and share information with intended agents in the traffic network. Predictions on the traffic conditions were made based on the information provided by community users (drivers and road links) and artificial neural networks. Particularly, the predicted time for a user to traverse a certain link was the outcome. The AntTIS system defined conditions on links within the traffic network by keeping both the information provided by the community and artificial neural networks. The vehicles and infrastructure were two agents involved in the process. While vehicle agents shared information about the links they intended to use and when they would arrive at every link, the infrastructure agents provided the vehicle agents with predicted travel times on links. This would allow the vehicle agents to make reliable decisions. With a large scale case study, the interactions between the agents should be minimized, and this condition indicated two major challenges with this application. Vehicle agents should only share their intentions (information about the links they intend to use) with infrastructure agents, which were a part of the intended routes. Similarly, infrastructure agents could only share information with vehicle agents that intended to use these infrastructure agents (links). One-minute updating intervals for the 
routing intentions were used. Every vehicle agent updated its intention every minute and shared it with the intended infrastructure agents. Infrastructure agents kept the intentions for two minutes if they were not updated. Afterwards, the intentions were erased. Large networks have been used to illustrate the realistic impact of using the proposed framework. Trip distance and trip duration were the two performance indicators that were examined. The base-line case was a user equilibrium under user optimality. With regards to the results, the author examined two cases: undisturbed and disturbed scenarios. The undisturbed scenario was when the network and the demand were not changed. For the disturbed scenario, flow disturbances and network disturbances were applied. Flow disturbances were disturbances that modified the traffic demand. Network disturbances were disturbances in the supply side of the network. The major findings were as follows. In the case of undisturbed, travel time experienced by the vehicles was slightly higher than the base-line case. The lower travel time in the latter case was due to the complete information about the network compared to the former case. The travelled distance was almost equal to the base-line case. For the disturbed scenario when AntTIS was adopted, the travel time was less compared to the base-line case, while the distance travelled was negligibly different from the base-line case. For both scenarios, the participation rate (market penetration rate) of vehicles sharing their intentions had a significant effect on the prediction quality (Claes, 2015). It is worth mentioning that travel time was the only routing objective in the aforementioned proposed anticipatory framework.

Pan et al. (2013) proposed proactive re-routing strategies to reduce travel time. The authors estimated congestion based on a density/jam density ratio. The update in their simulation was event-based and speed was estimated based on the Greenshield model. Linear relationship between density and speed was adopted, which is a major limitation. The actual relationship between speed and density is not $100 \%$ linear. When the density was very low, speed underestimation was the outcome (Papacostas and Prevedouros, 1993). The authors found that their re-routing performed as good as the dynamic traffic assignment (Pan et al., 2013). 
Liang and Wakahara (2014) also applied re-routing based on predictions for the traffic congestion. One of the developed predictive models was based on the spatiotemporal correlation. The authors assumed that the amount of traffic was constant during each prediction time interval, which was unrealistic and did not reflect on traffic conditions efficiently (Liang and Wakahara, 2014).

S. Liu and Q. Qu (2016) developed a crowdsourcing dynamic congestion model to apply anticipatory routing for a fleet of cooperative vehicles by predicting the probability distribution of traffic conditions. A one-minute prediction interval was used, and data points were obtained from the GPS traces and social media. The authors found that their approach outperformed the conventional routing approach (S. Liu and Q. Qu, 2016).

It can be concluded that frameworks for the anticipatory routing application have been proposed. There were strong indications that the anticipatory routing was associated with a positive impact on the network performance with respect to the myopic routing case. Nevertheless, the unsophisticated prediction models did not analyze the effect of the anticipatory routing strategy reliably and realistically. Moreover, the compliance was a serious issue in some of the cases and would be overcome if CAVs were adopted. In the previous research, travel time was the only routing objective, and anticipatory routing was applied in a centralized routing framework. Thus, this chapter overcomes the drawbacks of the previous related work and applies anticipatory multi-objective eco-routing in a distributed routing framework, E2ECAV. A large urban network, which was based on downtown Toronto, was utilized despite the associated complexity. To consider the high resolution data points, a microscopic level of aggregation was applied for the traffic and emission models. Finally, sophisticated prediction models were used to accurately reflect on the traffic and environmental status. 


\subsection{Traffic and Emission Models}

For a real-time application, high resolution data points were required. A microscopic agentbased traffic simulation was deployed for the anticipatory routing application. The IDM car following model (Treiber, Hennecke, and Helbing, 2000) was used to obtain the secondby-second acceleration. The second-by-second speed of vehicles was estimated based on the acceleration. Further details related to the traffic model and IDM can be found in Section 3.3. For a compatible level of aggregation with the microscopic traffic model, MOVES was the employed emission model (United States Environmental Protection Agency, 2014). The second-by-second traffic and environmental variables of every vehicle were stored. Then, at every one-minute prediction interval, the link cost, whether it was travel time and/or GHG, was predicted using the developed predictive models in Chapter 6 .

\subsection{Case Study}

Unlike most of the existing studies, a congested urban network based on downtown Toronto was selected as the case study. The network consists of 223 links and 76 centroids. The case study is associated with a high level of heterogeneity due to the following link characteristics: speed limit, number of lanes, and directions. Further details can be found in Section 3.5. Travel demand was obtained from the Transportation Tomorrow Survey (TTS) (DMG, 2011). The time dependent exogenous demand Origin-Destination (OD) matrices were based on 5 minute intervals from TTS (DMG, 2011). The demand during 2014 was estimated based on the provided TTS data that represents a congested traffic condition. The simulation ended once all of the vehicles reached their destinations. Similarly to the myopic routing application, in Chapter 5, 100\% CAVs was considered only. 


\subsection{Predictive Models}

To apply the anticipatory routing while optimizing TT, GHG, or TT\&GHG, predicted values were required. Unlike the previous work that predicted TT based on historical data, previous iterations of traffic simulations, or regression models (Bottom, 2000), this work utilized the sophisticated deep learning LSTM predictive models. The best prediction models, as shown in Chapter 6, were developed for the speed and GHG ER and were utilized for the anticipatory routing application in this chapter. The LSTM models were of two hidden layers, and their hyper-parameters were systematically tuned using Bayesian optimization (Krizhevsky, Hinton, et al., 2009) as illustrated in Section 6.5. For the GHG ER prediction, the link speed, density, GHG ER, and in-link speed of the three previous sequences were the used predictors as in Section 6.7.2. For the speed prediction, the three previous sequences of speed, density, and in-link speed were the utilized predictors as illustrated in Section 6.7.3. The RMSE of the GHG ER and speed predictive models were $0.36 \mathrm{~g} / \mathrm{s}$ and $5.95 \mathrm{~km} / \mathrm{h}$, respectively. During the simulation, at every minute $(t)$, the predictors of the last three sequences $(t-3)$ to $(t-1)$ were utilized to predict the cost at time $(t)$, and the cost was based on the routing objective, which was TT, GHG, or both. Physical constraints have been made for a realistic application. When either the predicted GHG ER or speed of time step $(t)$ was negative, the value was set to zero. The predicted speed of time step $(t)$ that surpassed the link speed limit was set to the link speed limit.

\subsection{Discussion}

The included routing strategies in this chapter are presented in Table 7.1. While the myopic routing strategies adopted the cost on the links at time interval $(t-1)$, the anticipatory routing was based on the predicted costs of the links at time $(t)$, whether it was TT, GHG,

or both TT\&GHG. The prediction interval was one minute (Alfaseeh, Djavadian, and Fa- 
rooq, 2018). The TT and GHG costs followed Equations 5.6 and 5.5, respectively. After investigating the different GHG costing approaches, the approximate vehicle cost of GHG traversing a link was the best costing approach as presented in Section 5.7.1. The approximate vehicle cost of GHG, as in Equation 5.5, required the predicted GHG ER and speed to forecast the time that would be spent on a studied link. The link cost was based on the predicted values. When TT was part of the routing objective, the predicted speed was used. When GHG was the routing objective, both the predicted speed and GHG ER were used. The general optimization process followed Equation 7.1. In the equation, $T T_{l}$ is travel time on link $l ; G H G_{l}$ is emissions on link $l$, which is the GHG (in $\mathrm{CO}_{2 \mathrm{eq}}$ ) in this case; $n$ is number of links on path $k$; and $W_{T T}$ and $W_{G H G}$ are the weights associated with travel time and GHG emissions, respectively. It is worth mentioning that variables in Equation 7.1 (TT and $G H G)$ have different units and weights were deployed to transform them into a consistent unit (e.g. monetary value) when multi-objective routing was applied. The adopted TT monetary value was $\$ 27.36 /$ hour based on Statistics Canada (CBC, 2019). The used monetary value of GHG emission was $\$ 15.77$ /Ton based on The World Bank (2019).

$$
\min \left\{\sum_{l=1}^{n} W_{T T} \cdot T T_{l}+\sum_{l=1}^{n} W_{G H G} \cdot G H G_{l}\right\}
$$

Based on Law and McComas (1991), every routing strategy ran for five trials with different random seeds and the average of any performance indicator was taken to account for stochasticity. The performance indicators were average TT, average VKT, total produced GHG, and total produced NOx. To further analyze the outcomes of the anticipatory routing strategies, a network level analysis was conducted in addition to the path analysis of a random vehicle of the different routing objectives. A comparison with the myopic routing strategies was applied. 
Table 7.1 The Investigated Routing Strategies

\begin{tabular}{|c|c|}
\hline Scenario name & Routing type \\
\hline $\mathrm{TT}_{m}$ & Myopic \\
\hline $\mathrm{GHG}_{m}$ & Myopic \\
\hline $\mathrm{TT}_{4} \mathrm{GHG}_{m}$ & Myopic \\
\hline $\mathrm{TT}_{a}$ & Anticipatory \\
\hline $\mathrm{GHG}_{a}$ & Anticipatory \\
\hline $\mathrm{TT}_{2} \mathrm{GHG}_{a}$ & Anticipatory \\
\hline
\end{tabular}

\subsubsection{Average TT, Average VKT, Total GHG, and Total NOx by the Different Routing Strategies}

Figure 7.2 demonstrates that anticipatory routing strategies outperformed the myopic strategies, regardless of the routing objective or performance indicator. The justification is threefold. Firstly, unlike the myopic routing, the anticipatory routing took into account the evolution of the traffic and environmental conditions. In other words, the link cost was based on the predicted value at time step $(t)$, while in the myopic case $(t-1)$ was the time step of the used cost. Secondly, the efficiency of the predictive models had an explicit impact on the performance of the anticipatory routing. Lastly, the representative GHG costing approach, when GHG was part of the optimization process, contributed to the finding. The approximate vehicle cost of GHG following Equation 5.5 takes into account the GHG ER and spent time on a studied link. Particularly, $\mathrm{TT}_{a}, \mathrm{GHG}_{a}$, and TT\&GHG introduced reductions in the total GHG by $23 \%, 23 \%$, and $18 \%$ compared to $\mathrm{TT}_{m}, \mathrm{GHG}_{m}, \mathrm{TT}_{\mathrm{TGHG}}$, respectively. These results show the positive impact of using anticipatory routing.

The same pattern of performance by the routing strategies was observed in the myopic and anticipatory routing applications. TT was the worst strategy followed by GHG and 
TT\&GHG. Whether it was myopic or anticipatory routing, when TT was the objective, all the possible paths in the network were assessed based on the travel time regardless of VKT, GHG, and NOx. When the network was congested, vehicles were distributed in the network to uncongested links, which triggered more VKT, GHG, and NOx. Links of low speed were replaced by links with a longer distance but a higher speed. On the other hand, whether routing was myopic or anticipatory when GHG was a part of the optimization process, a more obvious enhancement was noticed. This is due to the fact that the approximate vehicle cost of GHG took into account the speed, travel time, and distance variables into account contributing to results in favour. The best combination of speed, GHG ER, and VKT was used. Both excessively high or low speeds triggered greater GHG ERs due to the quasi-convex relationship between speed and GHG ER (Djavadian, Tu, et al., 2020). The links with a speed close to the optimal value were prioritized as long as the objective was optimized. The approximate vehicle cost of GHG minimizes VKT (re-routing), which contributes to less GHG emissions. With regards to the results, $\mathrm{TT}_{m}$ surpassed the $\mathrm{GHG}_{m}$ in terms of VKT, total GHG, and total NOx by $13 \%, 12 \%$, and $15 \%$, respectively. $\mathrm{TT}_{a}$ surpassed the $\mathrm{GHG}_{a}$ in terms of the VKT, total GHG, and total NOx by $1 \%, 11 \%$, and $8 \%$, respectively. The variations were reduced between the performance indicators of different routing objectives for anticipatory routing, and this was because the cost was predicted reflecting on the current situation efficiently.

For multi-objective routing with TT and GHG, the scale of the monetary value of GHG was different from TT. Hence, to make the costs comparable, a scaling factor of $10^{5}$ was used for GHG cost. The GHG cost on the links was taken by tons and time was in minutes in the optimization formula. Normalization can be another option to scale the values of TT and GHG in the optimization formula. When both TT and GHG were optimized and of a comparable scale, TT was minimized more strictly. Whether it is myopic or anticipatory, comparing TT\&GHG to TT is like comparing the SO to the UE traffic assignment. Details can be found in Section 2.1. The approximate vehicle cost of GHG in the TT\&GHG acted 


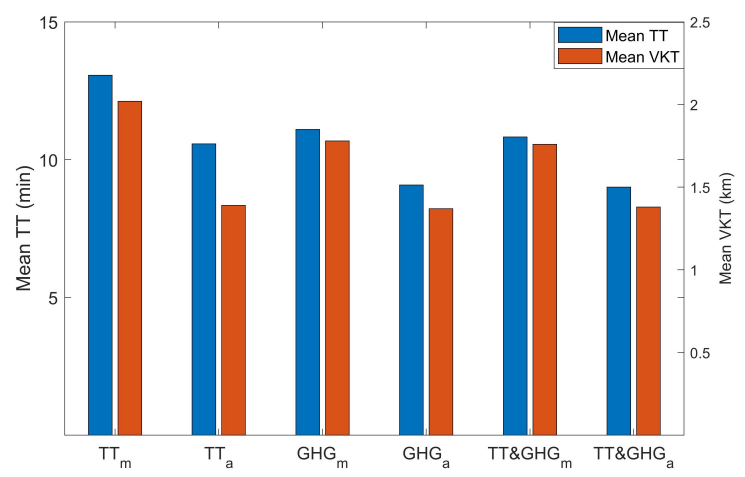

(a)

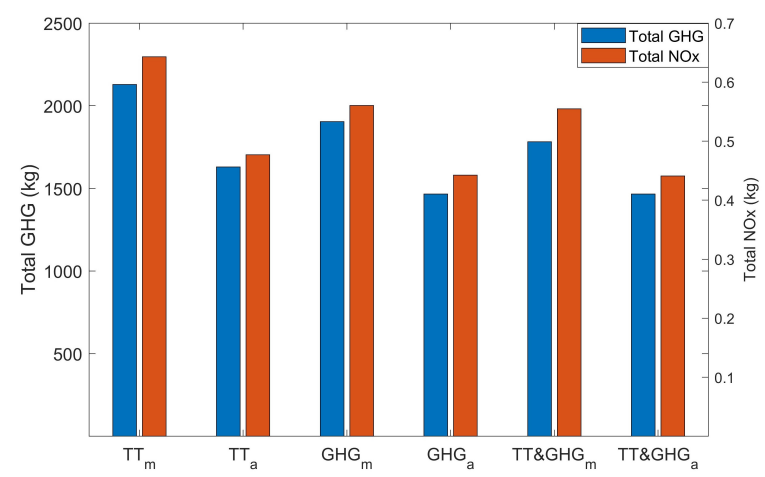

(b)

Figure 7.2 The Impact of Myopic and Anticipatory Routing Strategies on the a) Mean TT and VKT and b) Total GHG and NOx

as the social cost (Papacostas and Prevedouros, 1993) that controlled the TT cost and contributed to a favourable outcome. TT\& $\mathrm{GHG}_{m}$ was associated with reductions in the average TT, average VKT, total GHG, and total NOx of 17\%, 13\%, 16\%, and 14\%, respectively, compared to $\mathrm{TT}_{m}$. On the other hand, $\mathrm{TT} \& \mathrm{GHG}_{a}$ was associated with reductions in the average TT, average VKT, total GHG, and total NOx of 15\%,1\%,10\%, and 7\%, respectively, compared to $\mathrm{TT}_{a}$. The enhancements in the case of anticipatory routing was less compared to the myopic case. This was due to the adopted prediction in the former case. Whether it was myopic or anticipatory, TT\&GHG introduced slight reductions in terms of the whole performance indicators compared to when GHG was the routing objective. TT\&GHG ${ }_{m}$ was associated with reductions in the average TT, average VKT, total GHG, and total NOx of $2 \%, 1 \%, 6 \%$, and $1 \%$, respectively, compared to $\mathrm{GHG}_{m}$. The reductions in the mean TT, mean VKT, total GHG, and total NOx in the case of TT\&GHG $\mathrm{TH}_{a}$ were of $1 \%, 1 \%, 0.2 \%$, and 0.1\%, respectively, compared to $\mathrm{GHG}_{a}$, as shown in Figure 7.2. The negligible improvement in the case of TT\&GHG compared to GHG was due to the fact that GHG controls the TT variable indirectly. When TT\&GHG were optimized, TT was minimized extensively compared to when GHG was the only routing objective. Highlighting the difference between the best anticipatory routing strategy, $\mathrm{TT} \& \mathrm{GHG}_{a}$, and $\mathrm{TT}_{m}$ presents reductions in average TT, 
average VKT, total GHG, and total NOx of $17 \%, 22 \%, 18 \%$, and 20\%, respectively. Since NOx is very sensitive to aggressive driving, links with a higher speed are not favourable. When TT was the routing objective, links with a lower speed might be used because they were unoccupied. Nevertheless, the additional time and distance experienced in the network made the total NOX in the network higher compared to when GHG was the objective, which found the balance between speed, GHG ER, and distance. It can be concluded that the additional time and longer trips were essential triggers to increase GHG and NOx in the case of myopic and anticipatory routing.

\subsubsection{Path Analysis of the Different Myopic and Anticipatory Routing Strategies}

For this analysis, the paths of the myopic and anticipatory routing strategies of a randomly chosen vehicle were examined. This analysis investigated the dynamics at an extremely disaggregated level of one vehicle. Figure 7.4 shows the results by the anticipatory routing strategies, which was compared to the myopic routing results in Figure 7.3.

The first thing to note is that the paths of the myopic routing strategies were associated with significant re-routing compared to the paths of the anticipatory routing strategies. The main justification is that the myopic routing strategies depended on the traffic condition of the previous time step $(t-1)$ to define the cost of the links, while the anticipatory routing strategies employed the predicted values at time $(t)$ of the required indicators for the cost estimation on links. In other words, the evolution of the traffic and environmental conditions was captured in the anticipatory routing, contributing to the improved performance. Whether the routing was myopic or anticipatory when TT was the routing objective, the path was significantly longer than when GHG was optimized solely or when combined with TT. This assures that when TT was the routing objective, vehicles were sent to uncongested links in the network regardless of VKT, GHG, and NOx. Figure 7.4a illustrates that instead 


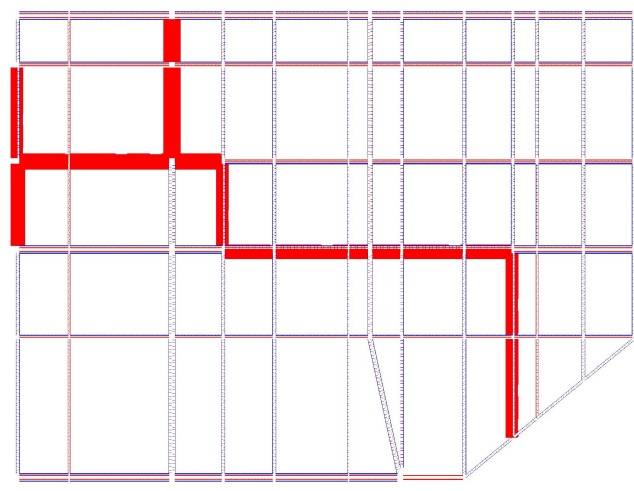

(a)

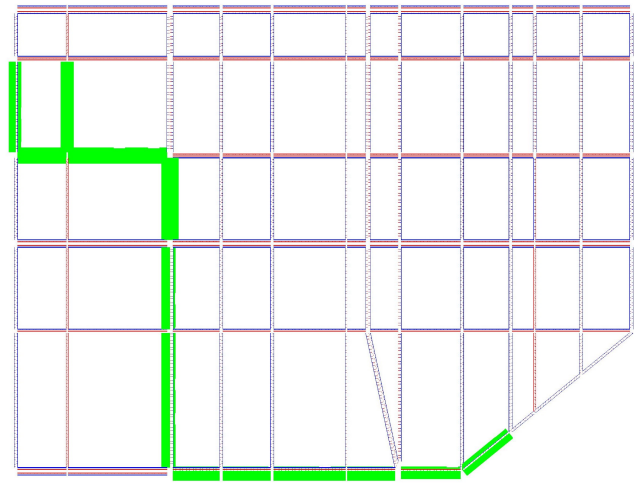

(b)

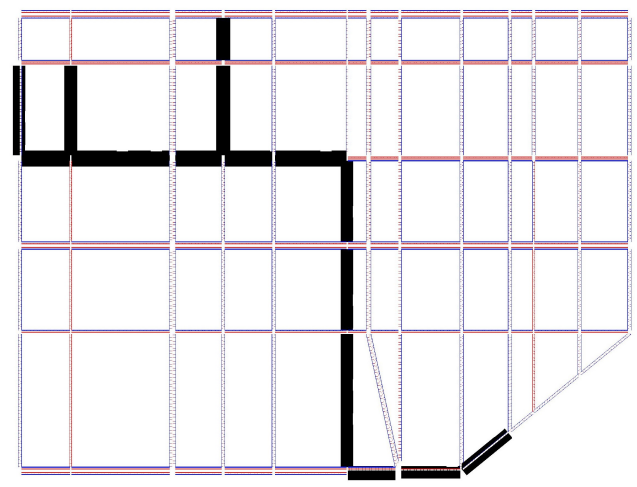

(c)

Figure 7.3 Myopic Routes of a Random Vehicle Subjected to Different Routing Strategies: a) TT, b) GHG, and c) TT\&GHG

of taking route $a$ (east-west) of two link, route $b$ (north-south) of five links was chosen. In the case of $\mathrm{TT}_{a}$, the vehicle traversed an additional distance of around 700 meters while taking route $b$ compared to $\mathrm{GHG}_{a}$ and $\mathrm{TT} \& \mathrm{GHG}_{a}$ routing strategies. The speed of route $b$ was around $38 \mathrm{~km} /$ hour, while the speed of route $a$ was around $1 \mathrm{~km} /$ hour. The shorter paths of $\mathrm{GHG}_{a}$ and $\mathrm{TT \&} \mathrm{GHG}_{a}$ routing strategies were due to the fact that the approximate vehicle cost of GHG takes into account the spent travel time as illustrated in Equation 5.5. Links of speed near to the optimal speed value, due to the quasi-convex relationship between speed and GHG ER (Djavadian, Tu, et al., 2020), were prioritized as long as the approximate vehicle cost of GHG was minimized. The best combination of speed, VKT, and GHG ER 


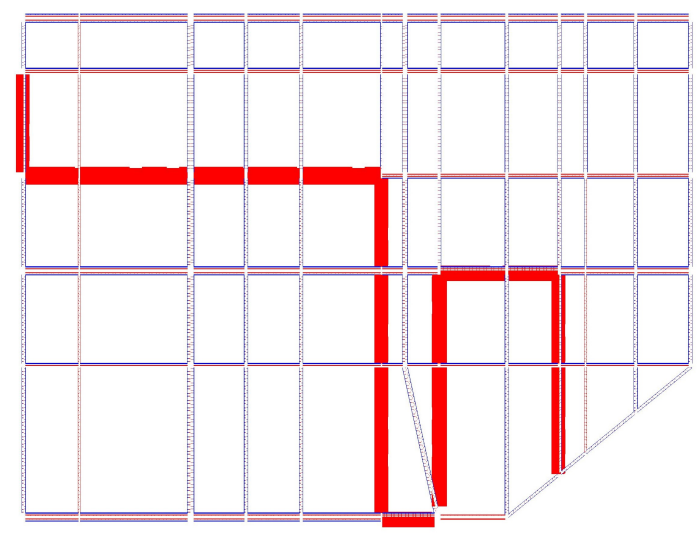

(a)

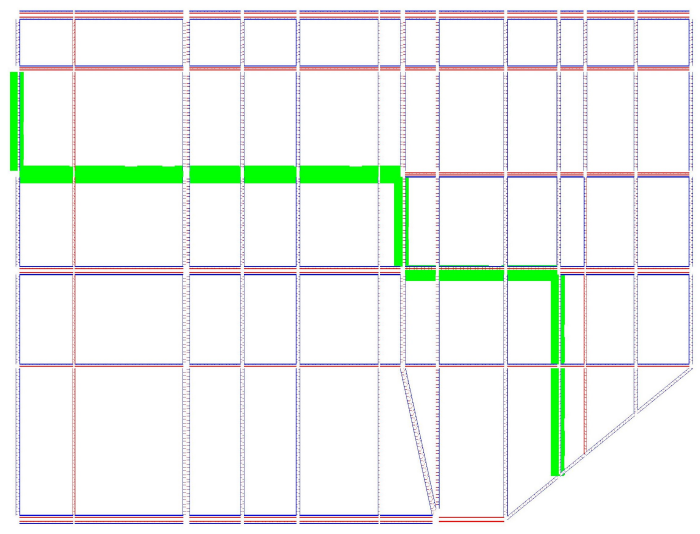

(b)

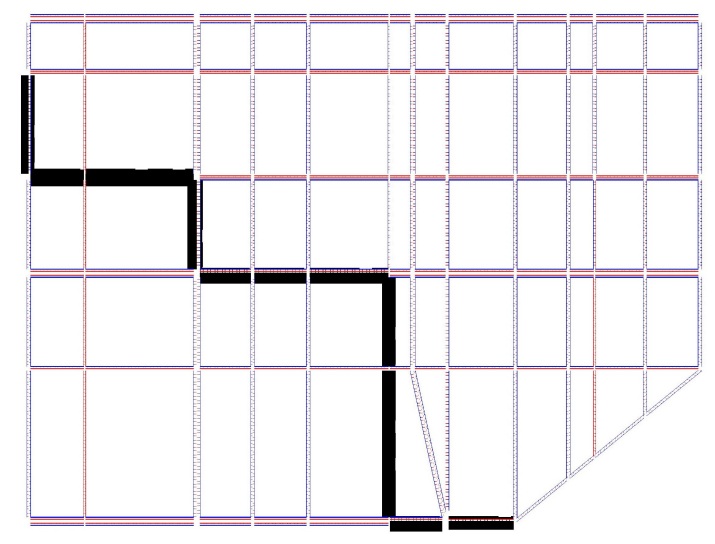

(c)

Figure 7.4 Anticipatory Routes of a Random Vehicle Subjected to Different Routing Strategies: a) TT, b) GHG, and c) TT\&GHG

was chosen to minimize the approximate vehicle cost of GHG. This explains why the trip length of the paths was less when GHG was a part of the optimization problem. The findings of this analysis are aligned with the mean TT, mean VKT related analysis in Section 7.6.1.

\subsubsection{Network Level Analysis of Average Speed, GHG, and NOx Over Time from the Routing Strategies}

Figures 7.5 and 7.6 present the average speed, produced GHG, and produced NOx over time of the myopic and anticipatory routing strategies, respectively. The first thing to note is 
that the network was loaded and unloaded quicker in the latter case compared to the former. $\mathrm{TT}_{a}, \mathrm{GHG}_{a}$ and $\mathrm{TT} \& \mathrm{GHG}_{a}$ required $13 \%, 10 \%$, and $10 \%$ less time to load and unload the network compared to $\mathrm{TT}_{m}, \mathrm{GHG}_{m}$, and $\mathrm{TT} \& \mathrm{GHG}_{m}$, respectively. The explanation is that the evolution of traffic and environmental conditions was not neglected and was reflected on the predicted costs of the links. This finding supports the results related to the average TT and average VKT in Section 7.6.1. As vehicles spent less time in the network and travelled shorter distances, in the case of the anticipatory routing, the network must be loaded and unloaded quicker. Furthermore, the noticed re-routing in the case of myopic in Figure 7.3 compared to the anticipatory in Figure 7.4 is another supporting analysis for why the network was loaded and unloaded quicker in the case of anticipatory routing strategies.

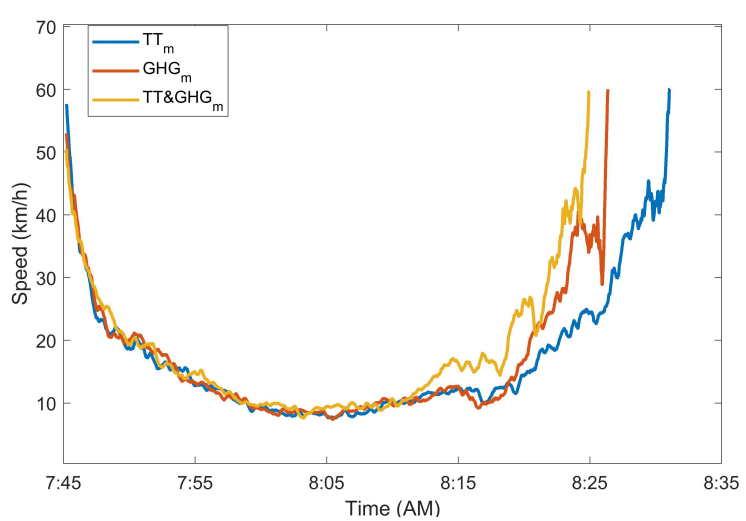

(a)

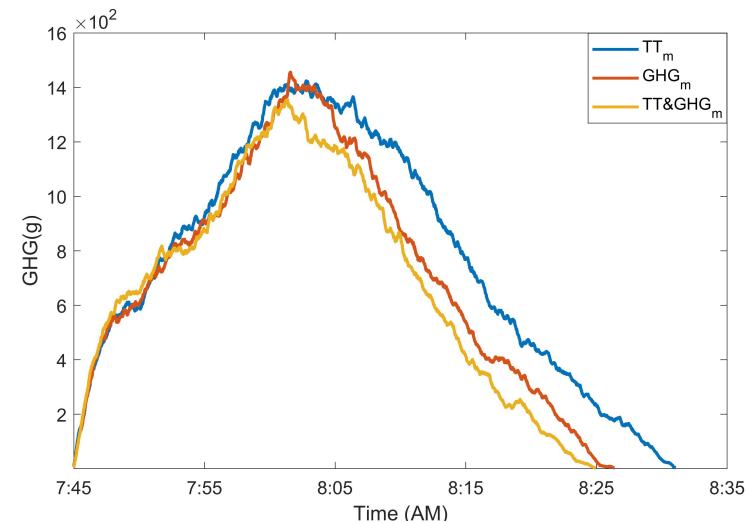

(b)

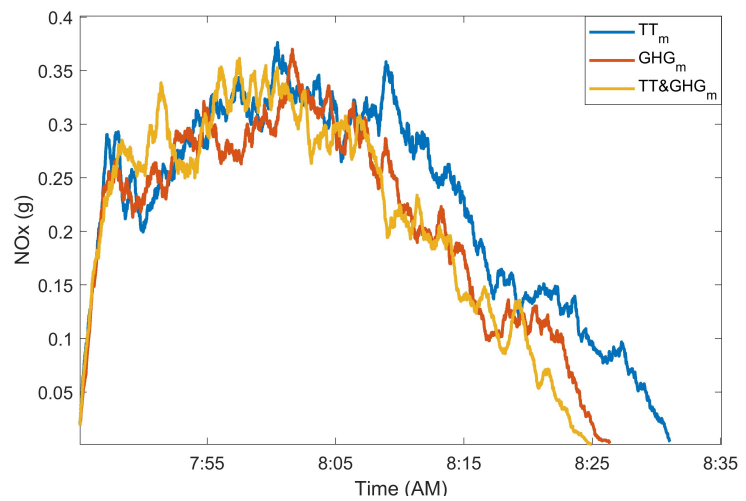

(c)

Figure 7.5 The Network Under the Different Myopic Routing Strategies and its a) Speed, b) GHG, and c) NOx Over Time 


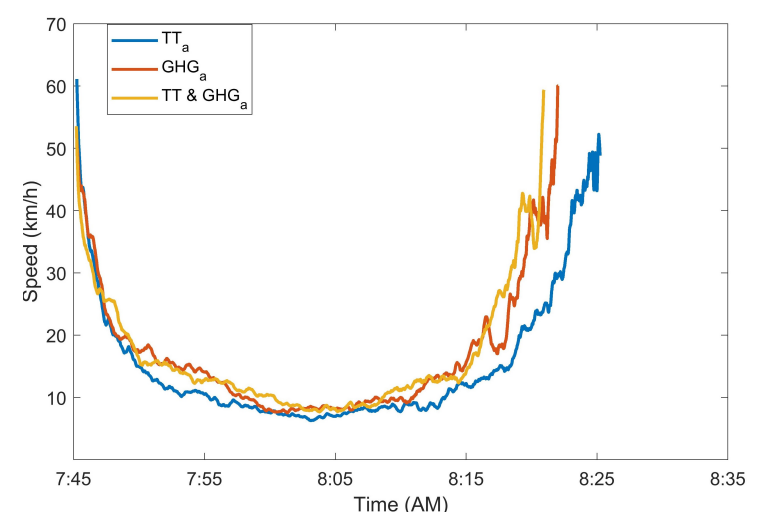

(a)

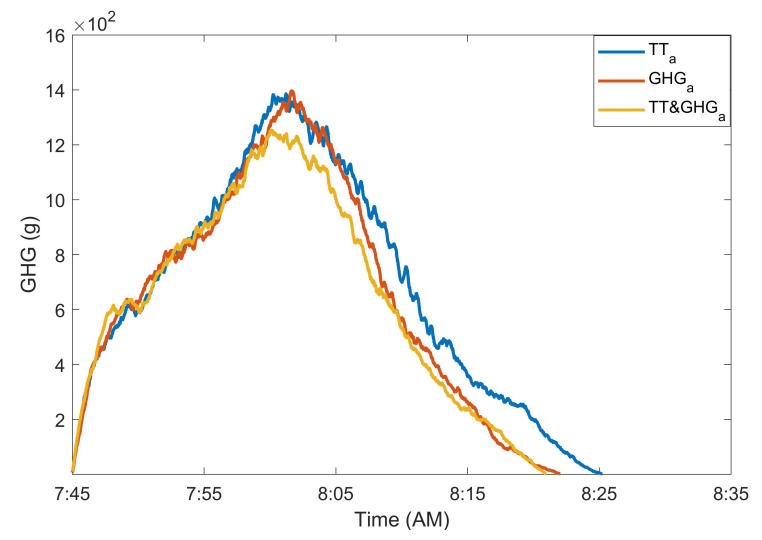

(b)

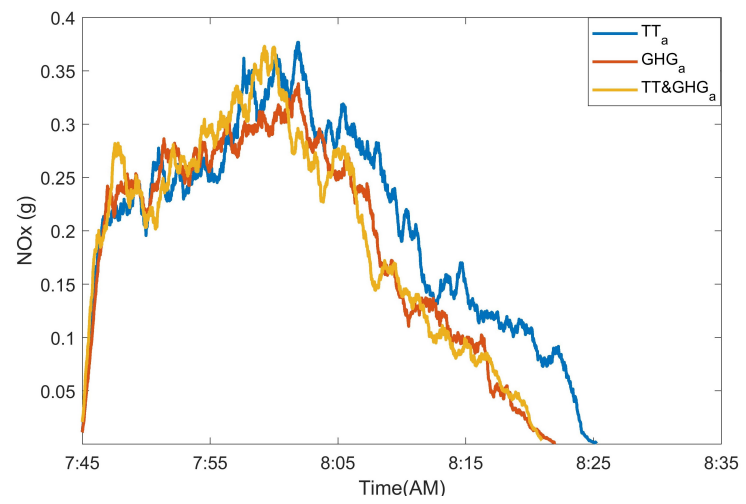

(c)

Figure 7.6 The Network Under the Different Anticipatory Routing Strategies and its a) Speed, b) GHG, and c) NOx Over Time

Figure 7.6 a illustrates that the time required to achieve $100 \%$ throughput in the case of $\mathrm{GHG}_{a}$ and TT\&GHG${ }_{a}$ was less by about $8 \%$ and $11 \%$, respectively, compared to $\mathrm{TT}_{a}$. The same pattern was noticed in $7.5 \mathrm{a}$ of the myopic routing strategies. Figure $7.6 \mathrm{a}$ shows that $\mathrm{TT}_{a}$ was associated with the lowest speed over time compared to $\mathrm{GHG}_{a}$ and $\mathrm{TT} \& \mathrm{GHG}_{a}$, which is the same pattern observed in Figure 7.5a of the myopic routing strategies. The main justification is that when TT was the objective, vehicles were distributed in the network in a way to minimize the TT regardless of any other variables, such as speed, VKT, and GHG. Vehicles were sent to uncongested links with a higher speed and triggered an increase in the VKT and time experienced in the network compared to GHG and TT\&GHG routing strategies. Hence, more vehicles were in the network over time in the case of TT compared 
to GHG and TT\&GHG, which contributed to the lower speed over time. When GHG was part of the optimization process, the approximate vehicle cost of GHG aimed at finding the best combination of speed and GHG ER, while preventing unnecessary re-routing. The approximate vehicle cost of GHG takes the speed and distance of the links implicitly and GHG ER explicitly. This means that for the same GHG ER and link length, links of higher speed were prioritized to be part of the optimal path as long as the GHG cost was minimized. The average speed of $\mathrm{GHG}_{a}$ and $\mathrm{TT} \& \mathrm{GHG}_{a}$ surpassed the average speed of $\mathrm{TT}_{a}$ by as much as $12 \mathrm{~km} / \mathrm{h}$.

The vehicles were loaded in the network at 7:45am and the congestion period was between 8:00am and 8:20am. Figure 7.6b of the anticipatory routing shows that the GHG values over time were very much comparable to the case of myopic routing as in Figure 7.5b. For both the myopic and anticipatory routing strategies, after 8:10am, the values of GHG varied over time with different routing objectives. The main explanation is the additional experienced TT and VKT vehicles.

The variation in terms of the performance indicators between the anticipatory routing strategies, namely $\mathrm{TT}_{a}, \mathrm{GHG}_{a}$, and $\mathrm{TT} \& \mathrm{GHG}_{a}$, was less compared to the myopic case, which was due to the proactive feature of the former. Figures $7.6 \mathrm{~b}$ and $7.6 \mathrm{c}$, of the anticipatory routing case, show that produced GHG and NOx over the time in the network were less when GHG was part of the optimization process. The justification is that vehicles experienced less time and travelled for shorter distances in the network as shown in Figure 7.2a. The decrease in the total GHG was more in the case of the anticipatory routing compared to the myopic due to the use of the predicted cost. NOx is very sensitive to aggressive driving (Alfaseeh, Djavadian, Tu, et al., 2019). This explains the high values at the beginning of the simulation in which vehicles travelled at the free flow speed. The TT and VKT were the two major factors contributing to the reduction in NOx over time regardless of the routing objective and type (myopic or anticipatory). This supports that NOx over time for myopic and anticipatory was less when GHG was optimized. It is noticed that there were a lot of fluctuations in the 
value of NOx over time in general as a result of the relationship between NOx and speed.

\subsection{Concluding Remarks}

Several studies proposed the great potential of using anticipatory routing to improve the performance of transport systems. Anticipatory routing frameworks have been developed to suite the real-time routing application. However, they were applied in centralized routing frameworks, utilized the VMS, and did not use efficient prediction models. In this chapter, the anticipatory multi-objective eco-routing was applied. Instead of using the cost of links at time $(t-1)$, the predicted cost at time $(t)$ was employed with different routing objectives, namely TT, GHG, and TT\&GHG. Unlike the existing related work, the anticipatory routing was applied while adopting the following: a distributed routing framework, microscopic level of aggregation for both the traffic and emission models, large urban case study, prediction interval of 1 minute, and sophisticated deep learning predictive models based on the LSTM. Anticipatory routing means that the evolution of the traffic and environmental conditions is captured and routing is proactive compared to the myopic, which is reactive. The enhancements were significant with the former compared to the latter in terms of the whole performance indicators and for the three routing strategies, TT, GHG, and TT\&GHG. The reductions in the mean $\mathrm{TT}$ of $\mathrm{TT}_{a}, \mathrm{GHG}_{a}$, and $\mathrm{TT} \& \mathrm{GHG}_{a}$ strategies were $19 \%, 18 \%$, and $17 \%$ compared to $\mathrm{TT}_{m}, \mathrm{GHG}_{m}$, and $\mathrm{TT} \& \mathrm{GHG}_{m}$, respectively. The justification of the significant decrease in TT in the anticipatory routing case was threefold. Firstly, the predictive feature in which the link cost (TT, GHG, or TT\&GHG) was estimated based on traffic conditions at time $(t)$ instead of $(t-1)$ in the case of myopic routing strategies. Within a minute, traffic and environmental states change due to the dynamic nature of transportation systems. If the link cost is underestimated compared to the real situation, vehicles would still be sent to congested links. On the other hand, if the link cost is overestimated, these links would be averted and not considered to be part of the optimal path. For anticipatory routing, 
traffic and environmental conditions were represented in a timely manner and reflected on the link cost. When congestion or any perturbation occurred in the network, the situation was captured, and the link cost was updated accordingly. Secondly, the high level of spatial and temporal resolution adopted by the predictive models and the link-cost updating process was a contributor to the reduced TT. Thirdly, the sophistication level of the predictive models was a significant trigger to the enhancements. The most representative predictors and number of sequences were chosen for a reliable model (LSTM) that captures the nonlinearity between the variables in concern. The network was loaded and unloaded quicker in the case of anticipatory routing. The decrease in the mean TT triggered reductions in the mean VKT of $31 \%, 23 \%$, and $22 \%$ for the $\mathrm{TT}_{a}, \mathrm{GHG}_{a}$, and $\mathrm{TT} \& \mathrm{GHG}_{a}$ routing strategies, respectively, compared to the myopic strategies. It can be concluded that minimizing TT and VKT introduced reductions in total GHG and NOx. The approximate vehicle cost of GHG took into account GHG ER explicitly, while link speed and distance implicitly contributing the aforementioned enhancements. Links with a speed close to the optimal value were prioritized as long as the GHG cost was minimized. Links with excessively high or low speeds were not considered, when they dissatisfied the optimization process. Too high speed links would contribute to increasing the GHG ER, while too low speed links would contribute to increasing GHG ER and travel time (Alfaseeh, Djavadian, Tu, et al., 2019). Considering a large urban network as the case study made this work scalable and realistic. However, since the data points were obtained from simulation, it would be an added value to apply this framework on real data from sensors. 
Chapter Eight

Summary, Concluding Remarks, and

\section{Future Outlook}




\subsection{Summary}

The overall goal of this dissertation was to examine different innovative routing solutions while considering the environmental aspect and taking advantage of connectivity in a realistic setting (high resolution data points and large case study). This dissertation started by summarizing the current state-of-the-art of the proposed eco-routing approach to minimize the negative impact that transportation systems impose on the environment. The impact of employing the dynamic distributed routing system, namely E2ECAV, was assessed to overcome the limitations with the existing centralized routing system. Based on the shortcomings found with the existing eco-routing applications, myopic multi-objective eco-routing was applied in the efficient distributed routing framework, E2ECAV, while adopting a high level of spatial (link level) and temporal resolution (with a one-minute updating interval). Due to the emergence of ICT technologies in transportation systems, data availability will not be an obstacle for many applications, including the prediction of traffic behaviour. Predictive models were developed in this dissertation to forecast the routing objectives, and these models will pave the way to replace the current reactive routing with proactive routing for potential enhancements to both the traffic and environmental characteristics. Predictive models were developed with the Long Short-Term Memory (LSTM), a deep learning method. The developed predictive models were used for the anticipatory routing application. Finally, anticipatory multi-objective eco-routing and myopic routing strategies were applied in a case study under similar settings to make a proper comparison. This work contributes to four major areas: routing frameworks, multi-objective routing, predictive models, and anticipatory routing. Figure 8.1 illustrates the stages for the current state of routing to the routing state in the near future, where more advancements of ICT will be deployed. The following summarizes each chapter in this dissertation.

This research was motivated by the impact transportation systems have on the environment. The first chapter of this dissertation provided an overview of this problem and 


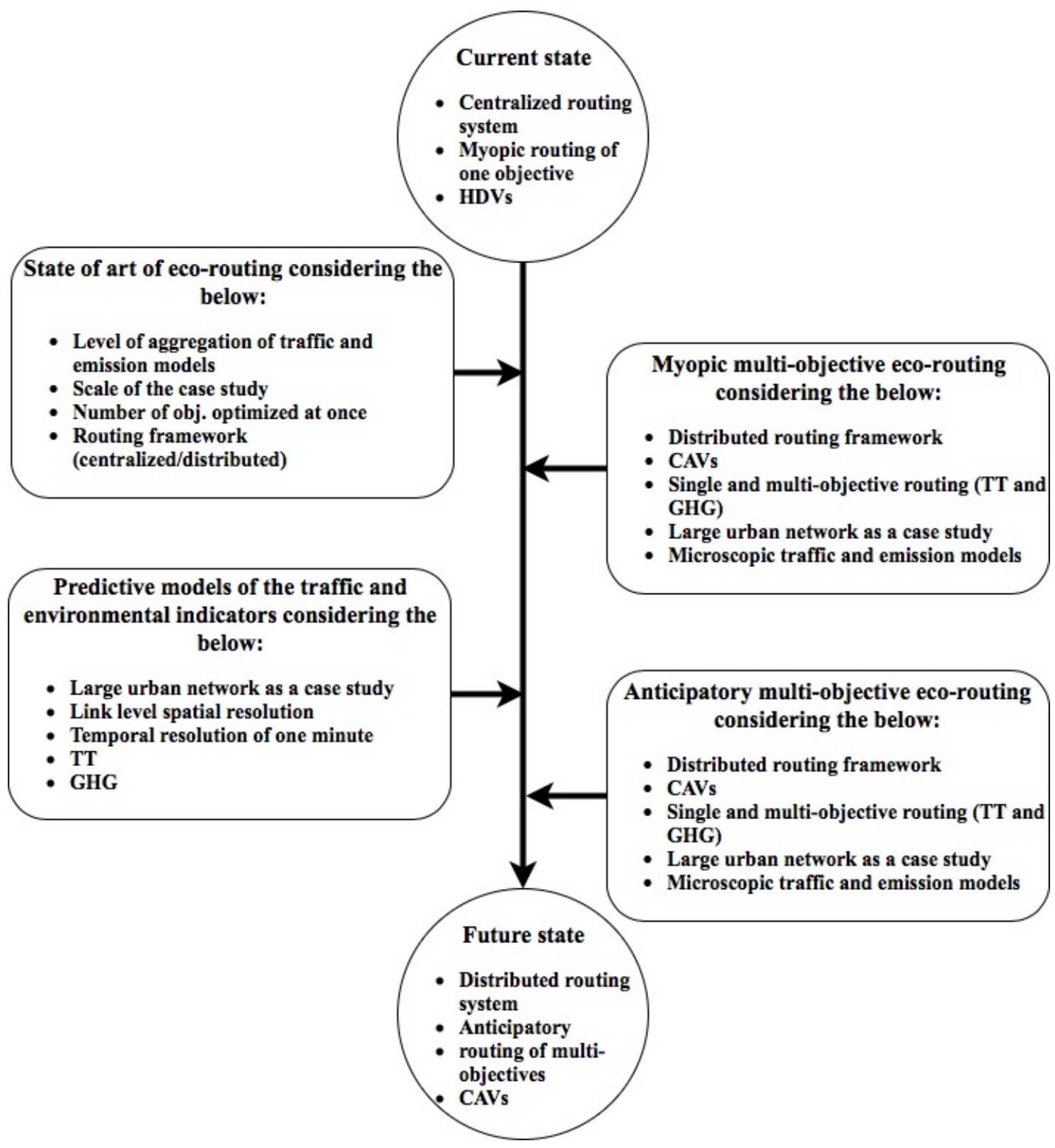

Figure 8.1 Summary of the Stages Followed in this Dissertation From the Current Routing State to the Routing State in the Near Future

proposed potential solutions. Furthermore, the contributions and research significance of this study were demonstrated. The first chapter also outlined its following chapters in this dissertation.

The second chapter of this dissertation investigated the state-of-the-art of eco-routing 
offered by other studies. A three factor taxonomy was proposed based on the most representative indicators for the performance of eco-routing models. The three factors were chosen as they were important characteristics of every eco-routing application. The first factor was the level of aggregation, which can be categorized as microscopic, mesoscopic, and macroscopic, for the traffic and emission models. To increase the efficacy of any transportation management process, high resolution data is required. The second factor was based on the scale of the case study, which is either large or small. This factor has a significant influence on the scalability of an eco-routing model. Finally, the third factor was the number of objectives optimized at once while routing vehicles to their destinations. Typically, one or two objectives are optimized at a time. For this three factor taxonomy, the strengths and weakness were illustrated for every category. For instance, macroscopic traffic and emission models were limited by their level of resolution, which affected the accuracy of estimations for the cost of a link. On the other hand, microscopic traffic and emission models increased the level of resolution but were associated with a high level of complexity. Another aspect was the size of the case study, which reflects on how generic the developed models are. Several contributions were provided from this chapter. The current state-of-the-art of eco-routing from recent studies asserted on good or poor practices for future applications. Based on the proposed three-factor taxonomy, this chapter also illustrated the expectations from the modifications on the routing process. As every eco-routing application is unique with its settings, the developed taxonomy implies the best choice for the three factors in every case. The findings of this chapter triggered the research work in the following chapters.

The third chapter defined the mutual components that were used in this dissertation. The description of the vehicle routing system was presented. The specifications were explained for the traffic and car-following model (IDM) applied in this study. The emission model, called MOVES, and its related formulas were discussed. The interactions between the routing framework, traffic simulator, and emission simulator were illustrated. Moreover, the case study and the characteristics of its major roads were described. 
The fourth chapter of this dissertation assessed the efficiency of the proposed dynamic distributed routing framework, E2ECAV, in the context of eco-routing. With E2ECAV, a microscopic agent-based traffic and emission simulation was adopted for the high-resolution data points. A comprehensive analysis was conducted at the network and link levels to examine and quantify the effect of E2ECAV on the traffic and environmental characteristics. The analysis considered different traffic conditions, in particular highly congested, congested, and uncongested, and market penetration rates (MPRs) of connected automated vehicles (CAVs), which included $0 \%, 5 \%, 30 \%, 50 \%, 70 \%$, and $100 \%$. The considered performance indicators were throughput, average TT, average VKT, flow, density, speed, total GHG emissions, and total NOx emissions. These indicators were used since they illustrate differences between various scenarios at the link and network levels.

The fifth chapter developed myopic single and multi-objective eco-routing strategies for a congested urban network. A section of downtown Toronto was selected as a large-scale and realistic case study to assess the performance of the eco-routing strategies. The efficient distributed routing system, E2ECAV, was used with CAVs to exploit the benefits of ICT advancements. Both travel time and GHG emissions were considered for the routing optimization process. This application was applied with 100\% MPR of CAVs. For a robust application, several GHG costing approaches were investigated, and the most representative approach was chosen. The results were analyzed at the link and network levels for a better comprehension of the events. Mean TT, mean VKT, total GHG emissions, and total NOx emissions were analyzed for different routing strategies and GHG costing approaches. The paths of a random vehicle subjected to the different routing strategies were extracted, and the characteristics of these paths were compared to better understand the behaviours of different routing objectives.

The sixth chapter demonstrates a major milestone and helps to make the application of anticipatory routing more realistic in practice. In this chapter, predictive models of the GHG emission rate (ER) and speed were developed. Before defining the most suitable predictive 
model, the three commonly used predictive models (ARIMA, clustering, and LSTM) were analyzed. The spatial and temporal aspects were the judging factors for the best model. A correlation analysis was conducted for the GHG ER and speed to define the highly correlated predictors (traffic and environmental variables). Predictors of the studied links were taken, but in-link indicators were also incorporated to better consider on the spatial dimension. For the LSTM, further improvements in the accuracy of predictions were demonstrated by using a deeper network and systematically tuning the hyper-parameters. It is of high importance to note that the developed predictive models are suitable for any urban network. This has been illustrated in the statistical analysis of traffic and environmental predictors. In the training dataset, speed ranged from 0 to $80 \mathrm{~km} / \mathrm{h}$, density ranged from 0 to $150 \mathrm{veh} / \mathrm{km} / \mathrm{ln}$, flow ranged from 0 to $8000 \mathrm{veh} / \mathrm{h}$, and GHG ER ranged from around 0.9 to $5 \mathrm{~g} / \mathrm{sec}$. That is, any urban network of indicators within the aforementioned ranges can be used for prediction purposes.

Finally, the seventh chapter applied anticipatory single and multi-objective eco-routing in similar settings as that of myopic eco-routing. Applying anticipatory routing means replacing the existing reactive (myopic) routing with proactive routing, which takes into account the evolution of the traffic and environmental state. Instead of relying on the cost of the links from the previous minute $(t-1)$, which is the case with myopic routing, the cost at the current minute $(t)$ was adopted with anticipatory routing. Based on the work related to anticipatory routing from the literature, simple predictive models were utilized because data was scarce and the routing process can become complex if the data has a high level of resolution. The developed sophisticated predictive models in Chapter 6 were used to predict in a timely manner speed and GHG ER of the different anticipatory routing strategies. The myopic and anticipatory routing protocol were compared based on the traffic and environmental aspects to present the potential enhancements of the proposed anticipatory routing strategy. The analysis was conducted at the link and network levels to understand the global and local impact of every routing strategy. 


\subsection{Concluding Remarks}

Due to the limitations of the centralized routing framework, E2ECAV dynamic distributed routing framework (Farooq and Djavadian, 2019) was proposed and assessed for its suitability for the eco-routing application. E2ECAV employed V2I and I2I communication in addition to CAVs. The results were promising since the traffic and environmental characteristics were enhanced with higher MPRs of CAVs. The benefit of E2ECAV was more noticeable when the network was congested or highly congested. When 100\% CAV was employed in a highly congested network, the mean travel time was reduced by as much as $41 \%$ compared to $100 \%$ human-driven vehicles (HDVs). However, this reduction in the average travel time was at the cost of the average VKT increasing slightly by less than $2.5 \%$. When the traffic condition was highly congested, the total GHG emissions were reduced by 39\% for $100 \%$ CAVs compared to $100 \%$ HDVs. The trend in mean travel time was similar to the trend observed in total GHG emissions. This illustrated the direct relationship between the time spent in the network and the emitted GHG. The encouraging positive findings of this chapter encourages the use of distributed routing systems in future research and practice.

Defining the state-of-the-art eco-routing was an essential step towards the final application of anticipatory routing. The important elements of the reviewed studies were organized into the developed three factor taxonomy. The three factors of the proposed taxonomy included the level of aggregation of the traffic and emission models, scale of the case study, and number of optimized objectives. These three factors were chosen, while classifying the existing eco-routing studies, due to their ability to reflect on three critical aspects: the level of data resolution, system scalability, and optimization objective(s). The developed taxonomy offered several major findings. The levels of aggregation for the traffic and emission models were generally at the macroscopic and mesoscopic level. Studies commonly optimized the routes with one objective due to the conflict when two or more objectives were optimized simultaneously. Improving throughput may have an adverse impact on produced GHG. Small 
case studies were selected, especially when a microscopic level of aggregation was used. Restrictions were made in terms of the OD matrix. A centralized routing framework was used while applying eco-routing. The findings of this part of this dissertation can be a guide for researchers and practitioners as the strengths and weaknesses of different settings for routing were demonstrated. The limitations found in the existing eco-routing studies were the motivation for several components in this dissertation, which should also trigger future research to offer better performing transportation systems.

Myopic single and multi-objective routing strategies were developed in the efficient distributed routing framework, E2ECAV. Among the different GHG costing approaches, the approximate vehicle cost of GHG was the most representative. The multi-objective strategy, which was to minimize travel time and GHG emissions, outperformed the single objective routing strategies, which minimized solely travel time or GHG emissions. When travel time was the routing objective, vehicles were distributed in the network to uncongested links to minimize travel time regardless of VKT. When GHG emissions were included in the optimization process, the best combination of GHG ER, speed, and distance was chosen. Links of speed close to the optimal value (due to the quasi-convex relationship between speed and GHG ER) were prioritized as long as the routing objective was satisfied. Links with very low or too high speeds were averted as their speed increased the GHG ER. A low speed was not favourable as it increased the GHG ER and travel time. The experienced additional travel time and VKT in the network had a direct impact on the total GHG and NOx emissions. While comparing the multi-objective and single objective (travel time only) routing strategy, the multi-objective approach reduced the mean travel time, mean VKT, total GHG emissions, and total NOx emissions by $17 \%, 13 \%, 16 \%$, and 14\%, respectively. This outcome illustrates the benefit of balancing the objectives and applying multi-objective eco-routing.

Myopic routing can be replaced with anticipatory routing to take advantage of the developments in ICT and minimize the adverse impact of transportation systems on the environment. While applying the anticipatory routing, sophisticated predictive models were required 
for a more realistic application. Three predictive models, namely clustering, ARIMA, and LSTM, were examined, and the LSTM performed the best considering the spatial and temporal dimensions efficiently. While clustering considered the predicted value as a discrete variable, LSTM considered it a continuous variable and accounted for temporary changes in the traffic condition. ARIMA was not scalable compared to the LSTM. LSTM was used to develop two individual network predictive models for the GHG ER and speed, and the models were developed to be used dynamically. For a robust application, a detailed correlation analysis was conducted, and the optimal predictors and number of sequences (minutes) of predictors were defined. The traffic characteristics of the studied link and in-links were incorporated to better consider the impact of the spatial aspect. Traffic and environmental conditions of in-links at time (t-1) give an indication of what the situation would be on a studied link at time (t). Deeper LSTM networks for each of the responses introduced significant improvement in the prediction performance of the models. The hyper-parameters of the network were tuned in two stages: manual and systematic. Bayesian optimization was used for the systematic tuning and adopted the ranges of the hyper-parameters that were tuned manually. Over fitting was taken into account with the whole three predictive models. The best LSTM predictive models for the GHG ER and speed had a RMSE of $0.36 \mathrm{~g} / \mathrm{sec}$ and $5.95 \mathrm{~km} /$ hour, respectively. The accuracy of the GHG ER prediction model was lower due to the complicated and nonlinear relationship between the GHG ER and studied predictors, such as speed, flow, and density. These results would guide the practitioners to choose the most suitable predictive model for their situation. The reliable developed predictive models in this part of the dissertation can be used with real data. The developed predictive model can be further improved by following the measures presented in Section 8.3.

Finally, the anticipatory multi-objective eco-routing was applied with the distributed routing framework, E2ECAV, in a case study that was based on downtown Toronto. The results were alluring from the traffic and environmental perspectives. The anticipatory routing strategies outperformed the myopic routing strategies regardless of the routing objective. 
The network was loaded and unloaded quicker with anticipatory routing. Since the routing strategy could adopt to the changing traffic conditions, the travel time decreased within the network. The link cost was predicted by using the developed predictive models. The mean TT of anticipatory routing while minimizing TT, GHG, and TT\&GHG was respectively lower by $19 \%, 18 \%$, and $17 \%$ than the corresponding myopic routing strategies that optimized TT, GHG, and TT\&GHG. As a result of the reductions in the mean TT, reductions in the total GHG were noticed. Anticipatory routing strategies that optimized TT, GHG, and TT\&GHG respectively reduced the total GHG emissions by $23 \%, 23 \%$, and $18 \%$ compared to the myopic routing strategies. The positive benefits of using anticipatory multi-objective eco-routing were the outcome of employing the high level of spatial and temporal resolution; sophisticated predictive models; and connectivity between the elements of a transportation system, such as the vehicles, links, and intersections. Hence, future research is encouraged to extend and refine this routing strategy.

Several stakeholders may benefit from this work. Google Maps is used by the public; service providers, such as Uber and Lyft; and government agencies. Thus, Google Maps is encouraged to adopt several suggested solutions in this study. Myopic eco-routes can be included as other options for users. The trade-offs between the different routing strategies in terms of the expected time and distance should be illustrated to allow drivers to make their own decisions. This adoption has a significant economic impact. In the case of myopic routing, for around 2500 vehicles in downtown Toronto, it took around 40 minutes to load and unload the network when GHG was the routing objective. The total TT and GHG were reduced by 114 hours and $225 \mathrm{~kg}$, respectively, compared to when TT was the objective. Economically, this means that when GHG was the myopic routing objective, the consumed fuel was reduced by 98 litres by around 2500 vehicles in downtown Toronto for the approximately 40 minutes. Based on a conversion factor of fuel to $\mathrm{CO}_{2}$ by the Natural Resource Canada (Natural Resources Canada, 2014), approximately $2.3 \mathrm{~kg}$ of $\mathrm{CO}_{2}$ is produced by burning 1 L of gasoline. To further demonstrate the benefits of routing vehicles myopically by only 
optimizing for the GHG at the network level, it can be assumed that cars are using the network for 12 hours per day for 25 days per week. While a reduction of 98 litres, compared to when TT is the objective, is noticed for around 2500 vehicles in the network for around 40 minutes, 147, 1764, 44100, and 529200 liters would be reduced per hour, day, month, and year, respectively. Similarly, the yearly reductions in total TT and GHG produced by only 2500 vehicles in downtown Toronto are of 615,600 hours and 1,215 tons, respectively. To even replace the myopic routing with the anticipatory routing, the predictive models developed in this study can be the starting point. Anticipatory routing would provide a higher positive impact on the environment and economy. When TT anticipatory routing is applied for around 2500 vehicles travelling in downtown Toronto, reductions within 35 minutes in the total TT and GHG of 115 hours and $500 \mathrm{~kg}$, respectively, were observed compared to the TT myopic routing strategy. For anticipatory routing, regardless of the routing objective, transportation networks were emptied faster. This approach helps to accommodate more vehicles while dealing with the increased vehicular demand for the same transportation supply. For anticipatory routing while TT is the objective, the network required 35 minutes to be loaded and unloaded. The effect of this application at the network level is substantial. Within a year, reductions in the total TT, GHG emissions, and consumed fuel would be 709,714 hours, 308,6 tons, and 1,339,200 litres, respectively, compared to routing myopically while optimizing TT. Citizens should be given the option to mitigate the negative impact of their chosen route on the environment and economy.

According to the New York Taxi and Limousine Commission report (TLC, 2020), Uber has the largest number of ride-hailing trips per day compared to Lyft and other similar service providers. Uber is encouraged to incorporate the eco-routing and anticipatory ecorouting concepts by giving their users a choice to optimize their routes. This consideration will offer mutual benefits as the routing would reduce the costs for Uber drivers and make Uber a more attractive service. By incorporating myopic eco-routing options, anticipatory routing, or eco-routing options, the direct outcome would be less fuel consumption, lower 
TT, and less emissions. A collaboration between governments and Uber or other similar service providers can be made while incentives are considered. It is worth mentioning that Google, Waze, Uber, and Lyft provide UE routing solutions, while the E2ECAV distributed routing system gives a SO solution. It was found in this dissertation that the E2ECAV contributed to reductions in TT, GHG, and NOx. That is, incentives should be given to users when their routes are a bit longer to achieve SO.

Government agencies in Canada are urged to step forward and consider innovative solutions, as presented in this research, that can alleviate the negative impact of transportation systems on the environment. This research demonstrates substantial incentives for governmental agencies in Canada to employ the proposed solutions. When drivers spend less time in the network, the following benefits can be received: the transportation network is used less, less fuel is consumed, less GHG is produced, and a higher demand is accommodated. Bus routes can be modified in a way to adopt any of the beneficial routing strategies (eco-routing, anticipatory, or anticipatory eco-routing). Minor changes in bus routes could contribute to a significant positive impact to the economical and environmental aspects. Since the variable message sign (VMS) is still commonly used to guide drivers to faster routes, eco-routing options can be incorporated to introduce improvements. Furthermore, instead of using the myopic cost of specific routes, the anticipated cost is proposed to be used since it can benefit from advancements in ICT. Governmental transportation entities can help researchers by providing real-time data to examine the developed framework in this study and other similar studies. Moreover, the GHG costing approach at link level can be a reference for policy makers. In this study, it was found that the approximate vehicle cost of GHG was the most efficient costing approach. It considers both GHG ER and TT of a studied link. The approximate vehicle cost of GHG can be the reference for a step forward to define the best method to estimate GHG cost at link level.

To summarize, there are different ways to reduce the adverse impact transportation systems impose on the environment. Routing should incorporate environmental variables 
and anticipate the changing traffic conditions. These features will help reduce the cost for commuters, benefit the economy of nations, and also reduce the contribution to global warming.

\subsection{Future Outlook}

This dissertation has demonstrated the impact of anticipatory routing while adopting several forms of ICT, CAVs and sensing. Nevertheless, there is still room for more research that can further enhance topics related to the anticipatory routing concept. The suggested topics for future research are below.

Training data: The data collected for training the predictive models can be more reflective on the wide range of traffic and environmental conditions. The performance of the prediction model and anticipatory routing can be improved by generating data points through a simulation or by collecting more measurements of low frequency traffic conditions among the data gathered. When data points of specific traffic and environmental conditions are of low frequency, more data points are required for a better performing predictive model. If simulation is the tool, scenarios that mimic specific conditions of low frequency in the trained data, such as low speed, high speed, low GHG ER, or high GHG ER, should be generated. If the data points were extracted from real measurements, situations that represent the low frequency conditions should be detected to extract the data needed to assess on the specific traffic and/or environmental states. Data points can be included to reflect on the whole possible traffic and/or environmental conditions, and this data will improve the prediction accuracy, especially for the LSTM. The LSTM network is trained to predict based on a training data set. When the set does not represent all the possible conditions, the prediction accuracy will be affected negatively. Furthermore, the simulated data points can be replaced with real data from sensors for a more realistic representation and assessment of the developed 
predictive models and proposed routing framework. Different sensors can be used for collecting data. There are three commonly used sensors for traffic control purposes, and the three types of sensors collect and share traffic data in different ways with the processing unit. 1) Passive magnetic sensors are installed on streets and are connected by wires or wirelessly with the processing units. 2) Pneumatic tube sensors are placed across the street and share traffic data with processing units through wired/wireless media. Finally, 3) inductive loops are wire coils buried into streets and send data to processing units (Guerrero-Ibáñez, Zeadally, and Contreras-Castillo, 2018).

Case study related: Researchers are encouraged to deploy a larger case that includes highways. Meshkani, Djavadian, and Farooq (2019) expanded the adopted case study in this dissertation to include the complete downtown area and used E2ECAV to develop a decentralized shared CAV system. Their case study can be adopted to apply the developed framework of anticipatory multi-objective eco-routing from this dissertation. Nevertheless, the predictive models should be adjusted to accommodate for the heterogeneity in the characteristics of the links.

Predictive models: Links can be categorized based on their main features, such as speed limit, number of lanes, and number of directions. This may contribute to greater accuracy for predictions, and the suitable predictive model will be chosen based on traffic and/or environmental characteristics of every link. To improve further the proposed predictive models, higher spatial and temporal resolution data points can be adopted. As the developed predictive models predicted only the next time step, it is proposed to develop predictive models for the whole considered predictors to make prediction for more than one time step possible. Being able to predict link cost at any future time step means that the anticipatory routing cost would be based on the expected time step to traverse a studied link.

Application related: For a more realistic application, the impact of public transporta- 
tion and freight is urged to be captured. It would be an added value to the literature to assess the impact of different MPRs of CAVs of different traffic condition for the myopic and anticipatory mult-objective eco-routing strategies. The utilization of intelligent vehicles will be gradual in the near future. Thus, assessing the impact of a wide range of MPRs of CAVs is urged. The V2I and I2I communications, which were adopted in E2ECAV, introduced substantial improvement from the traffic and environmental perspectives. Examining the effect of $\mathrm{V} 2 \mathrm{~V}$ communication is also an essential aspect to tackle. The V2V communication is applied by using the dedicated shortrange communications (DSRC), which are one- or two-way short to medium range wireless communication channels. The DSRC communications are developed specifically for automotive use (Miller, Shaw, et al., 2001). In the case of V2V utilization, it is expected that upstream vehicles would disseminate information about their destination and receive information about traffic and/or environmental conditions from downstream vehicles to estimate the optimal path while taking into account the updating interval and routing objective. Adopting V2V communication would reduce the need to adopt sensors and equipped infrastructure. V2V communication would contribute to a substantial reduction in the required capital investment to apply myopic and anticipatory routing strategies efficiently. Another suggestion for future research is that the efficiency of the developed models and the proposed framework can be illustrated by utilizing real-time measurements from sensors. Since E2ECAV adopts the First-In-First-Out (FIFO) logic at intersections, it is preferable to consider the real situation where vehicles are controlled by traffic lights at intersections. Another aspect to take into account is to make E2ECAV more realistic by representing the lateral movement, while Gipps (Hidas, 2002) and MOBIL (Kesting, Treiber, and Helbing, 2007) are proposed models to investigate. Anticipatory routing strategies can be incorporated into the personal navigation platforms to reduce TT, GHG, and NOx while routing vehicles to their destinations. It is expected that HDVs and other types 
of intelligent vehicles, such as automated vehicles (AVs) and CAVs, will be electric vehicles $(\mathrm{EVs})$ in the future. Although EVs are associated with zero tailpipe emissions, EVs contribute to energy consumption and air pollution during the generation of electricity adopted to charge EVs. In the case of EVs, the concept of energy regeneration is highly correlated with acceleration and deceleration, which contributes to more energy consumption (Hu, J. Wu, and Schwanen, 2017). In future research related to routing whether the vehicular technology is conventional or electric, eco-driving at the operational level should be taken into account for less emissions. Eco-driving at the operational level aims to minimize idling, choose the optimal speed, use cruise control feature, and avert aggressive driving behaviour (Sivak and Schoettle, 2012). As NOx is the pollutant reflecting on the public health aspect and since speed has a direct impact on the produced NOx, future research is urged to investigate the optimal speed on highways and urban streets that would reduce the produced NOx but would not affect the traffic characteristics negatively. Since diesel is a major contributor to the produced NOx (Cox, 1999), vehicles that use diesel fuel should be taken into account in similar analysis. This would introduce a realistic estimation of the emitted GHG and NOx.

Optimization related: The constrained anticipatory routing is an important aspect to investigate for further potential enhancements. Travel time or GHG can be constrained to not exceed a threshold. Constrained eco-routing will demonstrate the trade-offs within a network and improve the comprehension of eco-routing based on different constraints. In 2009, about $50 \%$ of the total NOx was produced by heavy-duty vehicles (diesel) (Toronto Public Health, 2014). NOx is the pollutant reflecting on the public health aspect (Cox, 1999). Hence, more research work should consider NOx compounds while optimizing the routes for better air quality. Other environmental indicators should be incorporated, such as exposure. When multi-objective routing is applied, 
the impact of different weights can be assessed for further improvements. 


\section{REFERENCES}

Abdullah, Lazim and Herrini Mohd Pauzi (2015). "Methods in forecasting carbon dioxide emissions: a decade review". In: Jurnal Teknologi 75(1).

Aggarwal, Charu C (2018). "Neural networks and deep learning". In: Springer 10, pp. 978-3.

Ahn, Kyoungho and Hesham Rakha (2013). "Network-wide impacts of eco-routing strategies: a large-scale case study". In: Transportation Research Part D: Transport and Environment 25, pp. 119-130.

Alfaseeh, Lama, Shadi Djavadian, and Bilal Farooq (2018). "Impact of Distributed Routing of Intelligent Vehicles on Urban Traffic". In: 2018 IEEE International Smart Cities Conference (ISC2). IEEE, pp. 1-7.

Alfaseeh, Lama, Shadi Djavadian, Ran Tu, et al. (2019). "Multi-objective Eco-routing in a Distributed Routing Framework". In: 2019 IEEE International Smart Cities Conference (ISC2). IEEE, pp. 747-752.

Alfaseeh, Lama and Bilal Farooq (2020a). "Deep Learning Based Anticipatory Multi-Objective Eco-Routing Strategies for Connected and Automated Vehicles". In: Transportation Research Part D: Transport and Environment arXiv:2006.16472.

Alfaseeh, Lama and Bilal Farooq (2020b). "Multifactor Taxonomy of Ecorouting Models and Future Outlook". In: Journal of Sensors 2020.

Alfaseeh, Lama, Ran Tu, et al. (2020). "Greenhouse Gas Emission Prediction on Road Network using Deep Sequence Learning". In: Transportation Research Part D: Transport and Environment arXiv:2004.08286v1.

Amarpuri, Lakshay et al. (2019). "Prediction of CO2 emissions using deep learning hybrid approach: A Case Study in Indian Context". In: 2019 Twelfth International Conference on Contemporary Computing (IC3). IEEE, pp. 1-6.

Ameyaw, Bismark and Li Yao (2018). "Analyzing the Impact of GDP on CO2 Emissions and Forecasting Africa's Total CO2 Emissions with Non-Assumption Driven Bidirectional Long Short-Term Memory". In: Sustainability 10(9), p. 3110. 
Ameyaw, Bismark, Li Yao, et al. (2019). "Investigating, forecasting and proposing emission mitigation pathways for CO2 emissions from fossil fuel combustion only: A case study of selected countries". In: Energy policy 130, pp. 7-21.

Andersen, Ove et al. (2013). "Ecotour: Reducing the environmental footprint of vehicles using eco-routes". In: Mobile Data Management (MDM), IEEE 14th International Conference. Vol. 1. IEEE (2013), pp. 338-340.

Antanasijević, Davor Z et al. (2014). "Forecasting GHG emissions using an optimized artificial neural network model based on correlation and principal component analysis". In: International Journal of Greenhouse Gas Control 20, pp. 244-253.

Aziz, HM Abdul and Satish V Ukkusuri (2012). "Integration of environmental objectives in a system optimal dynamic traffic assignment model". In: Computer-Aided Civil and Infrastructure Engineering 27(7), pp. 494-511.

Bandeira, Jorge M et al. (2018). "Exploring multiple eco-routing guidance strategies in a commuting corridor". In: International Journal of Sustainable Transportation 12(1), pp. 5365.

Barth, Matthew J, Guoyuan Wu, and Kanok Boriboonsomsin (2015). "Intelligent transportation systems and greenhouse gas reductions". In: Current Sustainable/Renewable Energy Reports 2(3), pp. 90-97.

Bektaş, Tolga and Gilbert Laporte (2011). "The pollution-routing problem". In: Transportation Research Part B: Methodological 45(8), pp. 1232-1250.

Ben-Akiva, Moshe et al. (2001). "Network state estimation and prediction for real-time traffic management". In: Networks and spatial economics 1(3-4), pp. 293-318.

Benedek, Christine M and Laurence R Rilett (1998). "Equitable traffic assignment with environmental cost functions". In: Journal of transportation engineering 124(1), pp. 1622 .

Bottom, Jon Alan (2000). "Consistent anticipatory route guidance". PhD thesis. Massachusetts Institute of Technology.

Boulter, P et al. (2007). "Assessment and reliability of transport emission models and inventory Systems (ARTEMIS)". In: Final Report.

Box, George EP et al. (2015). Time series analysis: forecasting and control. John Wiley \& Sons.

California Air Quality Research Board (2014). User's Guide.

CBC (2019). urlhttps://www.cbc.ca/news/business/statistics-canada-commute-times-study1.5038796. [Online; accessed 19-April-2019]. 
Cherkassky, Boris V, Andrew V Goldberg, and Tomasz Radzik (1996). "Shortest paths algorithms: Theory and experimental evaluation". In: Mathematical programming 73(2), pp. 129-174.

Chiu, Yi-Chang et al. (2011). "Dynamic traffic assignment: A primer". In: Transportation Research E-Circular( E-C153).

Claes, Rutger (2015). "Anticipatory Vehicle Routing”. PhD thesis. KU Leuven, Science, Engineering \& Technology.

Claes, Rutger and Tom Holvoet (2011). "Ant colony optimization applied to route planning using link travel time predictions". In: 2011 IEEE International Symposium on Parallel and Distributed Processing Workshops and Phd Forum. IEEE, pp. 358-365.

Claes, Rutger, Tom Holvoet, and Danny Weyns (2011). "A decentralized approach for anticipatory vehicle routing using delegate multiagent systems". In: IEEE Transactions on Intelligent Transportation Systems 12(2), pp. 364-373.

Council of Ministers Transportation and Highway Safety (2012). "The high cost of congestion in Canadian cities". In: Council of Ministers Transportation and Highway Safety.

Cox, Lyndon (1999). Nitrogen oxides (NOx) why and how they are controlled. Diane Publishing.

Dengiz, Asiye Özge, Kumru Didem Atalay, and Orhan Dengiz (2018). "Grey forecasting model for CO 2 emissions of developed countries". In: The International Symposium for Production Research. Springer, pp. 604-611.

Djavadian, Shadi, Lama Alfaseeh, et al. (2020). "Multi-Objective Eco-Routing in a Distributed Traffic Management Framework with a Case study of Downtown Toronto". In: 99 ${ }^{\text {th }}$ Annual Meeting of Transportation Research Board, Washington, DC. TRB.

Djavadian, Shadi and Bilal Farooq (2018). "Distributed Dynamic Routing Using Network of Intelligent Intersections". In: ITS Canada ACGM (2018).

Djavadian, Shadi, Ran Tu, et al. (2020). "Multi-Objective Eco-Routing for Dynamic Control of Connected \& Automated Vehicles". In: Transportation Research Part D: Transport and Environment, pp. 1-21.

DMG (2011). Transportation Tomorrow Survey.

Duan, Yanjie, Yisheng Lv, and Fei-Yue Wang (2016). "Travel time prediction with LSTM neural network". In: 2016 IEEE 19th International Conference on Intelligent Transportation Systems (ITSC). IEEE, pp. 1053-1058.

Elbery, Ahmed and Hesham Rakha (2019). "City-Wide Eco-Routing Navigation Considering Vehicular Communication Impacts". In: Sensors 19(2), p. 290. 
Elbery, Ahmed, Hesham Rakha, Mustafa Y ElNainay, et al. (2016). "Eco-routing: An Ant Colony based Approach". In: VEHITS (2016), pp. 31-38.

Elbery, Ahmed, Hesham Rakha, Mustafa Elnainay, et al. (2015). "Eco-routing using V2I communication: System evaluation". In: Intelligent Transportation Systems (ITSC), IEEE 18th International Conference on. IEEE (2015), pp. 71-76.

Elhenawy, Mohammed, Hao Chen, and Hesham Rakha (2014). "Dynamic travel time prediction using data clustering and genetic programming". In: Transportation Research Part C: Emerging Technologies 42, pp. 82-98.

Environment and Climate Change Canada (2020). Greenhouse gas emissions. https:// www . canada.ca/en/environment- climate-change/services / environmental-indicators / greenhouse-gas-emissions.html. [Online; accessed 28-Aug-2020].

EPA, USEPA (2020a). "Overview of Greenhouse Gases". In: Greenhouse gas emissions.

EPA, USEPA (2020b). "Sources of greenhouse gas emissions". In: Retrieved December.

Farooq, Bilal and Shadi Djavadian (2019). "Distributed Traffic Management System with Dynamic End to End Routing US Provisional Patent Service No 62865 725". In:

Fomunung, Ignatius, Simon Washington, and Randall Guensler (1999). "A statistical model for estimating oxides of nitrogen emissions from light duty motor vehicles". In: Transportation Research Part D: Transport and Environment 4(5), pp. 333-352.

Gass, Saul I (2003). Linear programming: methods and applications. Courier Corporation.

Gelbart, Michael A, Jasper Snoek, and Ryan P Adams (2014). "Bayesian optimization with unknown constraints". In: arXiv preprint arXiv:1403.560\%.

Gmira, Maha et al. (2017). "Travel speed prediction using machine learning techniques". In: Proc ITS World Congr. Pp. 1-10.

Green, Marc (2000). " How long does it take to stop?" Methodological analysis of driver perception-brake times". In: Transportation human factors 2(3), pp. 195-216.

Grote, Matt et al. (2018). "A practical model for predicting road traffic carbon dioxide emissions using Inductive Loop Detector data". In: Transportation Research Part D: Transport and Environment 63, pp. 809-825.

$\mathrm{Gu}$, Yuanli et al. (2019). "Short-term prediction of lane-level traffic speeds: A fusion deep learning model". In: Transportation research part C: emerging technologies 106, pp. 1-16.

Guerrero-Ibáñez, Juan, Sherali Zeadally, and Juan Contreras-Castillo (2018). "Sensor technologies for intelligent transportation systems". In: Sensors 18(4), p. 1212. 
Guo, Liya, Shan Huang, and Adel W Sadek (2013). "An evaluation of environmental benefits of time-dependent green routing in the greater Buffalo-Niagara region". In: Journal of Intelligent Transportation Systems 17(1), pp. 18-30.

Harding, John et al. (2014). Vehicle-to-vehicle communications: readiness of V2V technology for application. Tech. rep. United States. National Highway Traffic Safety Administration.

Hausberger, Stefan et al. (2003). "Emission factors for heavy-duty vehicles and validation by tunnel measurements". In: Atmospheric Environment 37(37), pp. 5237-5245.

Hawas, Yaser E and Hani S Mahmassani (1996). "Comparative analysis of robustness of centralized and distributed network route control systems in incident situations". In: Transportation Research Record 1537(1), pp. 83-90.

Hermans, Michiel and Benjamin Schrauwen (2013). "Training and analysing deep recurrent neural networks". In: Advances in neural information processing systems, pp. 190-198.

Hidas, Peter (2002). "Modelling lane changing and merging in microscopic traffic simulation". In: Transportation Research Part C: Emerging Technologies 10(5-6), pp. 351-371.

Hochreiter, Sepp and Jürgen Schmidhuber (1997). "Long short-term memory". In: Neural computation 9(8), pp. 1735-1780.

Hu, Kezhen, Jianping Wu, and Tim Schwanen (2017). "Differences in energy consumption in electric vehicles: An exploratory real-world study in Beijing". In: Journal of Advanced Transportation 2017.

Huang, Xianan and Huei Peng (2018). "Eco-Routing based on a Data Driven Fuel Consumption Model". In: arXiv preprint arXiv:1801.08602.

Huang, Yuansheng, Lei Shen, and Hui Liu (2019). "Grey relational analysis, principal component analysis and forecasting of carbon emissions based on long short-term memory in China". In: Journal of cleaner production 209, pp. 415-423.

Hutter, Frank, Jörg Lücke, and Lars Schmidt-Thieme (2015). "Beyond manual tuning of hyperparameters". In: KI-Künstliche Intelligenz 29(4), pp. 329-337.

INFRAS, AG (2004). "Handbook emission factors for road transport 3.2". In: Quick reference. Version 3.

Innamaa, Satu (2000). "Short-term prediction of traffic situation using MLP-neural networks". In: Proceedings of the 7th world congress on intelligent transport systems, Turin, Italy, pp. 6-9.

International Joint Commission (2012). "Canada/United States Air Quality Agreement: Progress report 2012". In: 
Ishak, Sherif and Ciprian Alecsandru (2004). "Optimizing traffic prediction performance of neural networks under various topological, input, and traffic condition settings". In: Journal of Transportation Engineering 130(4), pp. 452-465.

Jerath, Kshitij and Sean N Brennan (2012). "Analytical prediction of self-organized traffic jams as a function of increasing ACC penetration". In: IEEE Transactions on Intelligent Transportation Systems 13(4), pp. 1782-1791.

Kaufman, David E, Robert L Smith, and Karl E Wunderlich (1991). "An iterative routing/assignment method for anticipatory real-time route guidance". In: Vehicle Navigation and Information Systems Conference, 1991. Vol. 2. IEEE, pp. 693-700.

Kesting, Arne, Martin Treiber, and Dirk Helbing (2007). "General lane-changing model MOBIL for car-following models". In: Transportation Research Record 1999(1), pp. 86-94.

Kesting, Arne, Martin Treiber, Martin Schönhof, et al. (2008). "Adaptive cruise control design for active congestion avoidance". In: Transportation Research Part C: Emerging Technologies 16(6), pp. 668-683.

Kingma, Diederik P and Jimmy Ba (2014). "Adam: A method for stochastic optimization". In: arXiv preprint arXiv:1412.6980.

Koupal, J et al. (2005). "MOVES2004 Energy and Emissions Inputs". In: EPA, Planned publication in January.

Krizhevsky, Alex, Geoffrey Hinton, et al. (2009). "Learning multiple layers of features from tiny images". In:

Law, Averill M and Michael G McComas (1991). "Secrets of successful simulation studies". In: Proceedings of the 23rd conference on Winter simulation. IEEE Computer Society, pp. 21-27.

Lee, Joyoung and Byungkyu Park (2008). "Evaluation of route guidance strategies based on vehicle-infrastructure integration under incident conditions". In: Transportation research record 2086(1), pp. 107-114.

Liang, Zilu and Yasushi Wakahara (2014). "Real-time urban traffic amount prediction models for dynamic route guidance systems". In: EURASIP Journal on Wireless Communications and Networking 2014(1), p. 85.

Lin, Chiun-Sin, Fen-May Liou, and Chih-Pin Huang (2011). "Grey forecasting model for CO2 emissions: A Taiwan study". In: Applied Energy 88(11), pp. 3816-3820.

Lipton, Zachary C, John Berkowitz, and Charles Elkan (2015). "A critical review of recurrent neural networks for sequence learning". In: arXiv preprint arXiv:1506.00019.

Liu, Siyuan and Qiang Qu (2016). "Dynamic collective routing using crowdsourcing data". In: Transportation Research Part B: Methodological 93, pp. 450-469. 
Liu, Z et al. (2020). "Predictions and driving factors of production-based CO2 emissions in Beijing, China". In: Sustainable Cities and Society 53, pp. 101-109.

Long, Jiancheng et al. (2016). "Link-based system optimum dynamic traffic assignment problems with environmental objectives". In: Transportation Research Part D: Transport and Environment 60, pp. 56-75.

Luo, Lihua et al. (2016). "Real-time route diversion control in a model predictive control framework with multiple objectives: Traffic efficiency, emission reduction and fuel economy". In: Transportation Research Part D: Transport and Environment 48, pp. 332-356.

Ma, Xiaolei et al. (2015). "Long short-term memory neural network for traffic speed prediction using remote microwave sensor data". In: Transportation Research Part C: Emerging Technologies 54, pp. 187-197.

Mahmassani, Hani S (1994). "Development and testing of dynamic traffic assignment and simulation procedures for ATIS/ATMS applications". In:

Mane, Ajinkya S and Srinivas S Pulugurtha (2018). "Link-level Travel Time Prediction Using Artificial Neural Network Models". In: 2018 21st International Conference on Intelligent Transportation Systems (ITSC). IEEE, pp. 1487-1492.

Mann, Amandeep Kaur and Navneet Kaur (2013). "Review paper on clustering techniques". In: Global Journal of Computer Science and Technology.

McLaren, Joyce et al. (2016). Emissions associated with electric vehicle charging: Impact of electricity generation mix, charging infrastructure availability, and vehicle type. Tech. rep. National Renewable Energy Lab.(NREL), Golden, CO (United States).

Meshkani, Seyed Mehdi, Shadi Djavadian, and Bilal Farooq (2019). "A Decentralizd Shared CAV system Design and Application". In: 2019 Tenth Triennial Symposium on Transportation Analysis (TRISTAN). TRISTAN.

Metrolinx (2008). Costs of Roads Congestion in the Greater Toronto and Hamilton Area: Impact and Cost Benefit Analysis of the Metrolinx Draft Regional Transportation Plan.

Milanés, Vicente and Steven E Shladover (2014). "Modeling cooperative and autonomous adaptive cruise control dynamic responses using experimental data". In: Transportation Research Part C: Emerging Technologies 48, pp. 285-300.

Miller, Harvey J, Shih-Lung Shaw, et al. (2001). Geographic information systems for transportation: principles and applications. Oxford University Press on Demand.

Ministry of Transportation Ontario (2017). Statistics and Management Reporting.

Mukherjee, D (2014). "Effect of pavement conditions on rolling resistance". In: American Journal of Engineering Research 3(7), pp. 141-148. 
Natural Resources Canada (2014). Auto Smart.

Natural Resources Canada (2020). Fuel Consumption Guide.

NHTSA (2013). "US department of transportation releases policy on automated vehicle development". In: Policy, pp. 14-13.

Ntziachristos, Leonidas et al. (2000). "Copert iii". In: Computer Programme to calculate emissions from road transport, methodology and emission factors (version 2.1), European Energy Agency (EEA), Copenhagen.

Olia, Arash et al. (2018). "Traffic capacity implications of automated vehicles mixed with regular vehicles". In: Journal of Intelligent Transportation Systems 22(3), pp. 244-262.

Pan, Juan et al. (2013). "Proactive vehicular traffic rerouting for lower travel time". In: IEEE Transactions on vehicular technology 62(8), pp. 3551-3568.

Pananurak, Worrawut, Somphong Thanok, and Manukid Parnichkun (2009). "Adaptive cruise control for an intelligent vehicle". In: 2008 IEEE International Conference on Robotics and Biomimetics. IEEE, pp. 1794-1799.

Pao, Hsiao-Tien and Chung-Ming Tsai (2011). "Modeling and forecasting the CO2 emissions, energy consumption, and economic growth in Brazil". In: Energy 36(5), pp. 2450-2458.

Papacostas, Constantinos S and Panos D Prevedouros (1993). Transportation engineering and planning.

Pascanu, Razvan et al. (2013). "How to construct deep recurrent neural networks". In: arXiv preprint arXiv:1312.6026.

Patil, Gopal R (2016). "Emission-based static traffic assignment models". In: Environmental Modeling \& Assessment 21(5), pp. 629-642.

Pavlis, Yannis and Markos Papageorgiou (1999). "Simple decentralized feedback strategies for route guidance in traffic networks". In: Transportation science 33(3), pp. 264-278.

Poucin, Guilhem, Bilal Farooq, and Zachary Patterson (2018). "Activity patterns mining in Wi-Fi access point logs". In: Computers, Environment and Urban Systems 67, pp. 55-67.

Price, Di et al. (1997). Nitrogen oxides: Impacts on public health and the environment. Tech. rep. Environmental Protection Agency, Washington, DC (United States). Office of ...

Radojević, D et al. (2013). "Forecasting of greenhouse gas emissions in Serbia using artificial neural networks". In: Energy Sources, Part A: Recovery, Utilization, and Environmental Effects 35(8), pp. 733-740.

Rahman, Abdur and Md Mahmudul Hasan (2017). "Modeling and forecasting of carbon dioxide emissions in Bangladesh using Autoregressive Integrated Moving Average (ARIMA) models". In: Open Journal of Statistics 7(4), pp. 560-566. 
Rakha, Hesham, Kyoungho Ahn, and Kevin Moran (2012). "Integration framework for modeling eco-routing strategies: logic and preliminary results". In: International Journal of Transportation Science and Technology 1(3), pp. 259-274.

Rakha, Hesham, Kyoungho Ahn, and Antonio Trani (2003). "Comparison of MOBILE5a, MOBILE6, VT-MICRO, and CMEM models for estimating hot-stabilized light-duty gasoline vehicle emissions". In: Canadian Journal of Civil Engineering 30(6), pp. 1010-1021.

Ran, Xiangdong et al. (2019). "An LSTM-based method with attention mechanism for travel time prediction". In: Sensors 19(4), p. 861.

Reimers, Nils and Iryna Gurevych (2017). "Optimal hyperparameters for deep lstm-networks for sequence labeling tasks". In: arXiv preprint arXiv:170\%.06799.

Robert, Christian (2014). Machine learning, a probabilistic perspective.

Scora, George and Matthew Barth (2006). "Comprehensive Modal Emission Model (CMEM) Version 3.01 User's Guide". In: University of California Riverside Center for Environmental Research and Technology 23, p. 24.

Sha, Rui and Andy HF Chow (2019). "A comparative study of centralised and decentralised architectures for network traffic control". In: Transportation Planning and Technology 42(5), pp. 459-469.

Sivak, Michael and Brandon Schoettle (2012). "Eco-driving: Strategic, tactical, and operational decisions of the driver that influence vehicle fuel economy". In: Transport Policy 22, pp. 96-99.

Smit, Robin, Richard Smokers, and Elke Rabé (2007). "A new modelling approach for road traffic emissions: VERSIT+". In: Transportation Research Part D: Transport and Environment $12(6)$, pp. 414-422.

Snoek, Jasper, Hugo Larochelle, and Ryan P Adams (2012). "Practical bayesian optimization of machine learning algorithms". In: Advances in neural information processing systems, pp. 2951-2959.

Society of Automotive Engineers (2018). Levels of Driving Automation. https://www.sae. org/news/press-room/2018/12/sae-international-releases-updated-visual-chart-for-its$\backslash \mathrm{T} 1 \backslash$ textquotedblleftlevels- of-driving- automation $\backslash \mathrm{T} 1 \backslash$ textquotedblright-standard-forself-driving-vehicles. [Online; accessed 1-June-2020].

Srivastava, Nitish et al. (2014). "Dropout: a simple way to prevent neural networks from overfitting". In: The journal of machine learning research 15(1), pp. 1929-1958.

Sun, Jie and Henry X Liu (2015). "Stochastic eco-routing in a signalized traffic network". In: Transportation Research Procedia 7, pp. 110-128. 
Sun, Wei, Caifei Wang, and Chongchong Zhang (2017). "Factor analysis and forecasting of CO2 emissions in Hebei, using extreme learning machine based on particle swarm optimization". In: Journal of cleaner production 162, pp. 1095-1101.

Sweet, Matthias, Carly Harrison, and Pavlos Kanaroglou (2015). "Congestion Trends in the City of Toronto: 2011-2014". In: McMaster Institute for Transportation and Logistics, Hamilton, Ontario, Canada.

Szeto, WY, Xiaoqing Jaber, and SC Wong (2012). "Road network equilibrium approaches to environmental sustainability". In: Transport Reviews 32(4), pp. 491-518.

Talebpour, Alireza and Hani S Mahmassani (2016). "Influence of connected and autonomous vehicles on traffic flow stability and throughput". In: Transportation Research Part C: Emerging Technologies 71, pp. 143-163.

The World Bank (2019). urlhttps://carbonpricingdashboard.worldbank.org/map ${ }_{d} a t a$.

TLC (2020). Taxi and Ridehailing Usage in New York City.

Toronto Foundation (2020). Toronto's Vital Signs.

Toronto Public Health (2014). Path to Healthier Air: Toronto Air Pollution Burden of Illness Update.

Treiber, Martin, Ansgar Hennecke, and Dirk Helbing (2000). "Congested traffic states in empirical observations and microscopic simulations". In: Physical review E 62(2), p. 1805.

Tu, Ran, Lama Alfaseeh, et al. (2019). "Quantifying the impacts of dynamic control in connected and automated vehicles on greenhouse gas emissions and urban NO2 concentrations". In: Transportation Research Part D: Transport and Environment 73, pp. 142151.

$\mathrm{Tu}$, Ran, Islam Kamel, et al. (2018). "Development of a hybrid modelling approach for the generation of an urban on-road transportation emission inventory". In: Transportation Research Part D: Transport and Environment 62, pp. 604-618.

Tudor, Cristiana (2016). "Predicting the evolution of CO2 emissions in Bahrain with automated forecasting methods". In: Sustainability 8(9), p. 923.

Tzeng, Gwo Hshiung and Chien-Ho Chen (1993). "Multiobjective decision making for traffic assignment". In: IEEE Transactions on Engineering Management 40(2), pp. 180-187.

United States Environmental Protection Agency (2014). Motor Vehicle Emission Simulator (MOVES): User's Guide For MOVES2014a.

United States Environmental Protection Agency (2020). How does MOVES Calculate CO2 and CO2 Equivalent Emissions? https://www . epa.gov/moves / how - does - moves calculate-co2-and-co2-equivalent-emissions. [Online; accessed 9-July-2020]. 
US Environmental Protection Agency (2003). User's guide to MOBILE6. 1 and MOBILE6. 2 Mobile Source Emission Factor Model.

USEPA (2015). Exhaust Emission Rates for Light-Duty On-road Vehicles in MOVES2014: Final Report.

Van Arem, Bart, Cornelie JG Van Driel, and Ruben Visser (2006). "The impact of cooperative adaptive cruise control on traffic-flow characteristics". In: IEEE Transactions on intelligent transportation systems 7(4), pp. 429-436.

Vander Werf, Joel et al. (2002). "Effects of adaptive cruise control systems on highway traffic flow capacity". In: Transportation Research Record: Journal of the Transportation Research Board( 1800), pp. 78-84.

Vlahogianni, Eleni I, Matthew G Karlaftis, and John C Golias (2014). "Short-term traffic forecasting: Where we are and where we're going". In: Transportation Research Part C: Emerging Technologies 43, pp. 3-19.

Wang, Yi et al. (2018). "Dynamic traffic assignment: A review of the methodological advances for environmentally sustainable road transportation applications". In: Transportation Research Part B: Methodological.

Xiao, Lin, Meng Wang, and Bart van Arem (2017). "Realistic car-following models for microscopic simulation of adaptive and cooperative adaptive cruise control vehicles". In: Transportation Research Record: Journal of the Transportation Research Board( 2623), pp. 1-9.

Xu, Huaying (2006). "Decentralized traffic information system design based on inter-vehicle communications". PhD thesis. Citeseer.

$\mathrm{Xu}$, Yan et al. (2014). "Mesoscopic traffic simulation on CPU/GPU". In: Proceedings of the 2nd ACM SIGSIM Conference on Principles of Advanced Discrete Simulation, pp. 39-50.

Yang, $\mathrm{Xu}$ and Wilfred W Recker (2006). "Modeling dynamic vehicle navigation in a selforganizing, peer-to-peer, distributed traffic information system". In: Journal of intelligent transportation Systems 10(4), pp. 185-204.

Yao, Baozhen et al. (2017). "Short-term traffic speed prediction for an urban corridor". In: Computer-Aided Civil and Infrastructure Engineering 32(2), pp. 154-169.

Yildirimoglu, Mehmet and Nikolas Geroliminis (2013). "Experienced travel time prediction for congested freeways". In: Transportation Research Part B: Methodological 53, pp. 4563.

Zegeye, Solomon K et al. (2009). "Model-based traffic control for balanced reduction of fuel consumption, emissions, and travel time". In: Proceedings of the 12th IFAC Symposium on Transportation Systems (2009), pp. 149-154. 
Zeng, Weiliang, Tomio Miwa, and Takayuki Morikawa (2016). "Prediction of vehicle CO2 emission and its application to eco-routing navigation". In: Transportation Research Part C: Emerging Technologies 68, pp. 194-214.

Zhang, G Peter (2003). "Time series forecasting using a hybrid ARIMA and neural network model". In: Neurocomputing 50, pp. 159-175.

Zhang, Xiaoyan and John A Rice (2003). "Short-term travel time prediction". In: Transportation Research Part C: Emerging Technologies 11(3-4), pp. 187-210.

Zhou, Mofan, Xiaobo Qu, and Sheng Jin (2016). "On the impact of cooperative autonomous vehicles in improving freeway merging: a modified intelligent driver model-based approach". In: IEEE Transactions on Intelligent Transportation Systems 18(6), pp. 14221428.

Zhu, Meixin, Xuesong Wang, Andrew Tarko, et al. (2018). "Modeling car-following behavior on urban expressways in Shanghai: A naturalistic driving study". In: Transportation research part C: emerging technologies 93, pp. 425-445.

Zuurbier, Frank Stendert (2010). "Intelligent route guidance". PhD thesis. Delft University. 